\title{
Analysis of Cell Signaling Perturbations in Response to Chronic Localized Infections
}

Amber Nicole Prince

West Virginia University, aprince@mix.wvu.edu

Follow this and additional works at: https://researchrepository.wvu.edu/etd

Part of the Analytical Chemistry Commons

\section{Recommended Citation}

Prince, Amber Nicole, "Analysis of Cell Signaling Perturbations in Response to Chronic Localized Infections" (2021). Graduate Theses, Dissertations, and Problem Reports. 8145.

https://researchrepository.wvu.edu/etd/8145

This Dissertation is protected by copyright and/or related rights. It has been brought to you by the The Research Repository @ WVU with permission from the rights-holder(s). You are free to use this Dissertation in any way that is permitted by the copyright and related rights legislation that applies to your use. For other uses you must obtain permission from the rights-holder(s) directly, unless additional rights are indicated by a Creative Commons license in the record and/ or on the work itself. This Dissertation has been accepted for inclusion in WVU Graduate Theses, Dissertations, and Problem Reports collection by an authorized administrator of The Research Repository @ WVU.

For more information, please contact researchrepository@mail.wvu.edu. 


\title{
Analysis of cell signaling perturbations in response to chronic localized infections
}

\author{
Nicole Prince \\ Dissertation submitted to the \\ Eberly College of Arts and Sciences \\ at West Virginia University \\ in partial fulfillment of the requirements for the degree of
}

Doctor of Philosophy in

Chemistry

\author{
Jonathan W. Boyd, Ph.D., Chair \\ Timothy R. Nurkiewicz, Ph.D., Co-Chair \\ Peng Li, Ph.D. \\ John B. Mertz, Ph.D. \\ Stephen J. Valentine, Ph.D. \\ C. Eugene Bennett Department of Chemistry
}

Morgantown, West Virginia

2021

Keywords: Chronic Infection, Cytokines, Phosphoproteins, Cell Signaling, Network Analysis

Copyright 2021 Nicole Prince 


\title{
ABSTRACT
}

\section{Analysis of cell signaling perturbations in response to chronic localized infections}

\author{
Nicole Prince
}

The tissue-level response to pathogens involves an intricate series of signal transduction events, influenced by immune and healing mediators that alert the host to danger and eliminate the infection. Disruptions to normal signaling events can compromise the host's ability to respond and lead to the development of chronic infections that cannot be resolved without clinical intervention. Prolonged inflammation due to chronic infection can damage tissues and compromise healing processes, thus, the interactions of immune and healing mediators in signaling cascades are intimately linked to tissue health outcomes. Studying signaling networks relevant to these responses provided a more thorough understanding of localized tissue health to identify the drivers of disruptions to signaling cascades, and this knowledge can lead to the development of improved diagnostic and therapeutic biomarkers to combat chronic infections. The work presented here focused on elucidating the relationships between immune and wound healing factors in an in vivo rodent model and a clinical cohort to understand the tissue-level responses to chronic inflammation and infection. Specifically, extracellular inflammatory immune responses (i.e., cytokines and chemokines) related to intracellular signaling (i.e., phosphorylation of proteins) were investigated to identify alterations in native responses compared to those provoked by chronic inflammation and infection. Reponses in native tissues were compared to tissues with inflammatory and infectious stimuli to test if levels of immune related cytokines were elevated in response to chronic joint infections. Wound healing phosphoproteins were also included to look for shifts in wound healing-related processes across groups. Traditional statistical approaches and network analysis were used to dissect these complex biological datasets and identified drivers of network disruptions in response to inflammation and infection. The spatial analysis suggested that changes in biological responses were related to proximity to inflammation and infection, and the degree of response differed across spatial gradients, which demonstrated the ability for these chronic insults to affect disparate tissues in a clinically-relevant manner. The objective of this research and future related research is to facilitate new clinical strategies to combat chronic infection, and monitoring alterations to cell signaling pathways in this work highlighted the value of using network analysis to approach biological interrogation of signal disruptions related to these insults. 


\section{DEDICATION}

This dissertation is dedicated to my parents, Mark and Jeanie Prince, for their wisdom, unconditional support, and encouraging me to love learning. 


\section{ACKNOWLEDGEMENTS}

I would first and foremost like to thank my advisor and mentor, Dr. Jonathan Boyd, for his years of guidance, patience, and sharing knowledge. He has taught me the value of perseverance, creativity, and having fun in research. His mentorship has made me a better scientist and person, and he will always serve as a role model for the type of researcher I strive to be.

I am very grateful to my committee members, Dr. Tim Nurkiewicz, Dr. Stephen Valentine, Dr. Peng Li, and Dr. Blake Mertz for their expertise and encouragement. They provided me critical advice and supported me both as a student and as a person. I would also like to thank Dr. Matthew Dietz for all of his help throughout my graduate work. He provided me with a better perspective for the impact of this research and was both an exceptional collaborator and unofficial mentor.

To past and present Boyd group members, thank you for the social support, endless laughs, and genuine camaraderie. Alice Han, Maren Prediger, and Julia Penatzer, thank you for always lending an ear, going on lunch dates, and making the graduate school experience much more enjoyable. Thank you to Suzanne Danley for help with everything from writing grants to life advice. I will really miss you! To our newest group members, Misa Shaw, Cayla Lynch, and Mackey Newman, thank you for making my last few months of graduate school fun. I am truly excited to see all the great things you do in the Boyd group. 
To my best friend, Neel Sharma, I can never thank you enough for your encouragement. Thank you for giving me research advice, keeping me grounded, and being my biggest advocate. I could not have done this without you.

Last, but certainly not least, thank you to all my family and friends that supported me through all the ups and downs of graduate school, especially my parents, my brother Aaron, James Lee, Bill Feeney, Zach Sasiene, Clara Warner, Elizabeth Burgio, Steven Knowlden, Rob Crovak, and Rachel Keselring. Thank you for listening to my frustrations, celebrating with me during the good times, and reminding me to have fun. 


\section{TABLE OF CONTENTS}

Title Page

$\begin{array}{ll}\text { Abstract } & \text { ii }\end{array}$

Dedication $\quad$ iii

Acknowledgements $\quad$ iv

Table of Contents

List o Figures

List of Tables $\quad$ xiii

Chapter 1: Introduction to cellular signaling networks of infection and strategies for analysis

$\begin{array}{lll}1.1 & \text { Overview of biological cell signaling } & 2\end{array}$

1.1.1 Components of biological cell signaling 2

1.1.2 Interactions of cell signaling pathways 7

1.1.3 Protein phosphorylation in cell signaling 10

1.2 The immune response to infection 13

1.2.1 Acute immune response 13

1.2.2 Chronic infections and immune response 15

1.2.3 Bacterial hijacking of host cell signaling 16

1.3 Disruptions to cell signaling pathways observed in pathological conditions $\quad 18$

1.3.1 Periprosthetic joint infection (PJI) and immune signaling 18

1.3.2 Dysregulation of cell signaling in other conditions 22

1.4 Bioinformatics approaches to understand complex biological responses 23

1.4.1 Background on common network methodologies 24 
1.4.2 Databases and model repositories 25

1.4.3 Advanced mathematical network analysis approaches 27

$\begin{array}{lll}1.5 & \text { References } & 30\end{array}$

Chapter 2: Localized Cytokine Responses to Total Knee Arthroplasty and Total Knee

Revision Complications

$\begin{array}{lll}2.1 & \text { Introduction } & 44\end{array}$

2.2 Materials and methods $\quad 47$

$\begin{array}{lll}2.2 .1 & \text { Patient cohort } & 47\end{array}$

2.2.2 Collection of tissue samples 49

$\begin{array}{lll}\text { 2.2.3 Sample preparation } & 50\end{array}$

2.2.4 Cytokine measurement $\quad 51$

2.2.5 Statistical analysis $\quad 51$

$\begin{array}{lll}2.3 & \text { Results } & 53\end{array}$

2.3.1 Seven cytokines exhibited infection-specific elevations in concentration 54

2.3.2 Six cytokines exhibited implant-related elevations in concentration (Primary TKA vs. Aseptic/Septic TKR)

2.3.3 Quadratic discriminant analysis (QDA) cytokine profiles for TKA vs. TKR 58

2.3.4 Effects of age, sex, and BMI on cytokine concentrations 60

2.4 Discussion $\quad 62$

$\begin{array}{lll}2.5 & \text { Conclusions } & 65\end{array}$

$\begin{array}{lll}2.6 & \text { Acknowledgements } & 66\end{array}$

$\begin{array}{lll}2.7 & \text { References } & 66\end{array}$ 
3.1 Introduction

$\begin{array}{lll}3.2 & \text { Materials and methods } & 78\end{array}$

$\begin{array}{lll}3.2 .1 & \text { Animals } & 78\end{array}$

3.2.2 Experimental rat model of chronic localized infection 78

$\begin{array}{lll}\text { 3.2.3 Tissue collection } & 79\end{array}$

$\begin{array}{lll}\text { 3.2.4 Sample preparation } & 81\end{array}$

$\begin{array}{lll}\text { 3.2.5 Cytokine and phosphoprotein measurement } & 81\end{array}$

$\begin{array}{lll}\text { 3.2.6 Statistical analysis } & 82\end{array}$

3.3 Cytokine Analysis of Rodent Tissues Between Sham, Implant Only, and Inf+Implant Groups

3.3.1 Eight cytokines elevated in implant-related manner 84

3.3.2 Three cytokines showed infection-specific response 86

3.3.3 Quadratic discriminant analysis and heat maps of cytokine profiles $\quad 88$

3.4 Discussion of cytokine concentrations in response to implant and infection 90

3.5 Phosphoprotein and Ingenuity Pathway Analysis (IPA) to understand spatially-disparate responses

3.6 Methods for phosphoprotein and network analysis 96

3.6.1 Statistical analysis of phosphoproteins 96

3.6.2 Construction of IPA networks 96

3.7 Results of phosphoprotein data and IPA networks 97

3.7.1 Ten phosphoproteins were elevated in response to implant or infection 97 
3.7.2 Five phosphoproteins showed increased responses proximal or distal to the operative knee

3.7.3 IPA revealed differences in wound healing responses

102

3.8 Discussion of Wound Healing Network Analysis

105

3.9 Acknowledgements

107

3.10 References

108

Chapter 4: Impact of Cytokines and Phosphoproteins in Response to Chronic Joint Infection

$\begin{array}{lll}4.1 & \text { Introduction } & 119\end{array}$

4.2 Materials and methods 122

$\begin{array}{lll}\text { 4.2.1 Patient cohort } & 122\end{array}$

$\begin{array}{ll}\text { 4.2.2 Collection of tissue samples } & 124\end{array}$

$\begin{array}{lll}\text { 4.2.3 Sample preparation } & 125\end{array}$

4.2.4 Cytokine and phosphoprotein measurement 126

$\begin{array}{ll}\text { 4.2.5 Data processing and statistical analysis } & 129\end{array}$

4.2.6 Network evaluation with Ingenuity Pathway Analysis (IPA) 130

$\begin{array}{ll}\text { 4.2.7 Network centrality parameter analysis } & 130\end{array}$

$\begin{array}{lll}4.3 & \text { Results } & 131\end{array}$

$\begin{array}{lll}\text { 4.3.1 Relative spatial cytokine responses } & 131\end{array}$

$\begin{array}{lll}\text { 4.3.2 Relative spatial phosphoprotein responses } & 134\end{array}$

$\begin{array}{lll}\text { 4.3.3 } & \text { IPA-generated networks } & 138\end{array}$

$\begin{array}{lll}\text { 4.3.4 Normalized radiality of all } 30 \text { nodes } & 140\end{array}$ 
$\begin{array}{lll}4.4 & \text { Discussion } & 145\end{array}$

$\begin{array}{lll}4.5 & \text { Conclusion } & 151\end{array}$

$\begin{array}{lll}\text { 4.6 Acknowledgements } & 152\end{array}$

$\begin{array}{lll}4.7 & \text { References } & 152\end{array}$

Chapter 5: Conclusions and Future Directions: Translating Signal Transduction Research into Clinically-Relevant Tools

5.1 Localized vs. Systemic Investigations of Clinical Pathologies

5.3 New Technologies for Rapid Analysis 


\section{LIST OF FIGURES}

Figure 1. 1 Cellular Response to External Stimuli. 3

Figure 1. 2 Modes of cellular signal transduction. 4

Figure 1. 3 Interactions of Cell Signaling Pathways. 10

Figure 1. 4 Mechanism of phosphorylation and dephosphorylation. 11

Figure 1. 5 Bead-based ELISA. $\quad 22$

Figure 2. 1 Map of approximate tissue collection locations. $\quad 50$

Figure 2. 2 Seven cytokines showed infection-dependent elevation in localized tissues. $\quad 55$

Figure 2. 3 Six cytokines showed implant-related elevation in localized tissues that was not infection-dependent.

Figure 2.4 Quadratic discriminant analysis (QDA) revealed distinct groupings for primary TKA vs. TKR (aseptic or septic).

Figure 3. 1 Sampling locations for muscle samples collected at 21 days post-surgery. $\quad 80$

Figure 3. 2 IL-1 $\alpha$, IL-5, IL-10, IL-12p70, IL-13, GM-CSF, IFN- $\gamma$, and TNF- $\alpha$ concentrations in response to implant and infection.

Figure 3. 3 IL-1 $\beta$, IL-4, and IL-6 tissue concentrations in response to implant and infection. 87

Figure 3. 4 QDA analysis (a) and heat map of relative cytokine concentrations (b). 89

Figure 3. 5 Ten phosphoproteins showed elevated levels in response to implant and infection. 99

Figure 3. 6 Five phosphoproteins showed elevated levels proximal or distal to the opeartive knee site.

Figure 3. 7 Ingenuity Pathway Analysis (IPA)-generated networks.

Figure 3. 8 Comparison of top up- and down-regulated nodes compared to the operative knee.

Figure 4. 1 Map of approximate tissue collection locations.

Figure 4. 2 Relative cytokine levels measured in tissues from primary TKA, aseptic TKR, and septic TKR at adjacent tissue layer (ATL) and radial tissue layer (RTL) debridement depths. 133 
Figure 4. 3 Relative levels of phosphoproteins associated with the proliferative processes in acute wound healing.

Figure 4. 4 Relative phosphoprotein levels associated with cell migration processes in acute wound healing.

Figure 4. 5 Relative levels of pro-apoptotic and inhibitory wound healing phosphoproteins in acute wound healing.

Figure 4. 6 Ingenuity Pathway Analysis (IPA)-generated networks for primary TKA, aseptic TKR, and septic TKR groups based on cytokine and phosphoprotein datasets.

Figure 4. 7 Changes in significant nodes between groups for low radiality outcomes.

Figure A. 1 Cytokine profiles in localized tissues in infection-specific inflammation.

Figure A. 2 Cytokine profiles in localized tissues in implant-related inflammation.

Figure A. 3 Cytokine calibration curves for human inflammatory cytokines.

Figure B. 1 X-ray images of Sham group rats at 21 days post-surgery.

Figure B. 2 X-ray images of Impant Only group rats at 21 days post-surgery.

Figure B. 3 X-ray images of Inf+Impant group rats at 21 days post-surgery.

Figure B. 4 Cytokine profiles in localized tissues in infection-specific inflammation.

Figure B. 5 Cytokine calibration curves for rodent inflammatory cytokines. 


\section{LIST OF TABLES}

Table 2. 1 Patient Information.

Table 2. 2 Pairwise Pearson Correlation Values Between Cytokine Concentrations and Age, Sex, and BMI.

Table 3. 1 Clinically-Relevant Parameters at 21 Days Post-Surgery.

Table 4. 1 Patient Information.

Table 4. 2 Cytokine Targets Measured in Tissue Samples.

Table 4. 3 Phosphoprotein Targets Measured in Tissue Samples.

Table 4. 4 Top 2 IPA Networks for Primary TKA, Aseptic TKR, and Septic TKR Groups. 139

Table 4. 5 Normalized Radiality of Nodes in the ATL Layer.

Table 4. 6 Normalized Radiality of Nodes in the RTL Layer.

Table A. 1 Average cytokine concentrations in the adjacent tissue layer (ATL).

Table A. 2 Average cytokine concentrations in the radial tissue layer (RTL).

Table B. 1 Cytokine concentrations for Sham, Implant Only, and Inf+Implant groups.

Table B. 2 X-Ray Grading Scores for Sham (N=6), Implant Only (N=6), and Inf+Implant (N=6) Groups at Post-Operative Day 0 (POD-0) and POD-21. 


\section{Chapter 1}

Introduction to cellular signaling networks of infection and strategies for analysis 


\subsection{Overview of biological cell signaling}

\subsubsection{Components of biological cell signaling}

Cells respond to cues from their environments through relays of complex signaling networks, creating a path for adaptation towards survival, proliferation, or death. Understanding these signal transduction networks is crucial when studying complex biological responses, such as those observed in chronic inflammation and infection. Cell signaling was first observed in hormonal studies by Claude Bernard in 1855, who described that hormones released into the bloodstream produced effects in distant cells [1]. Bernard created a new concept of the "milieu intérieur" which described regulation through complex cellular feedback signaling to maintain homeostasis. Specifically, he described how interstitial fluid in the extracellular environment can provide a protective barrier for cells and tissues to preserve stability in response to stimuli. Since then, biological signaling has been pursued by a wide variety of biochemical researchers, and the term "signal transduction" as it is known now was first coined in the 1970s, providing the basis for the modern model of cell signaling, consisting of receptors, transducers, and amplifiers [2]. When a signal ligand binds to a receptor, this information is transduced into the intracellular space and amplified within the cell to produce a response, and examples of signal ligands include growth factors, hormones, cytokines, neurotransmitters, and many other small molecules. The release of these signals can reflect perturbations to the homeostatic state, and signal transduction is the primary means for communicating this information within and between cells. A simplified diagram of the incorporation of external stimuli to produce cell signaling changes in cells is shown in Figure 1.1. 


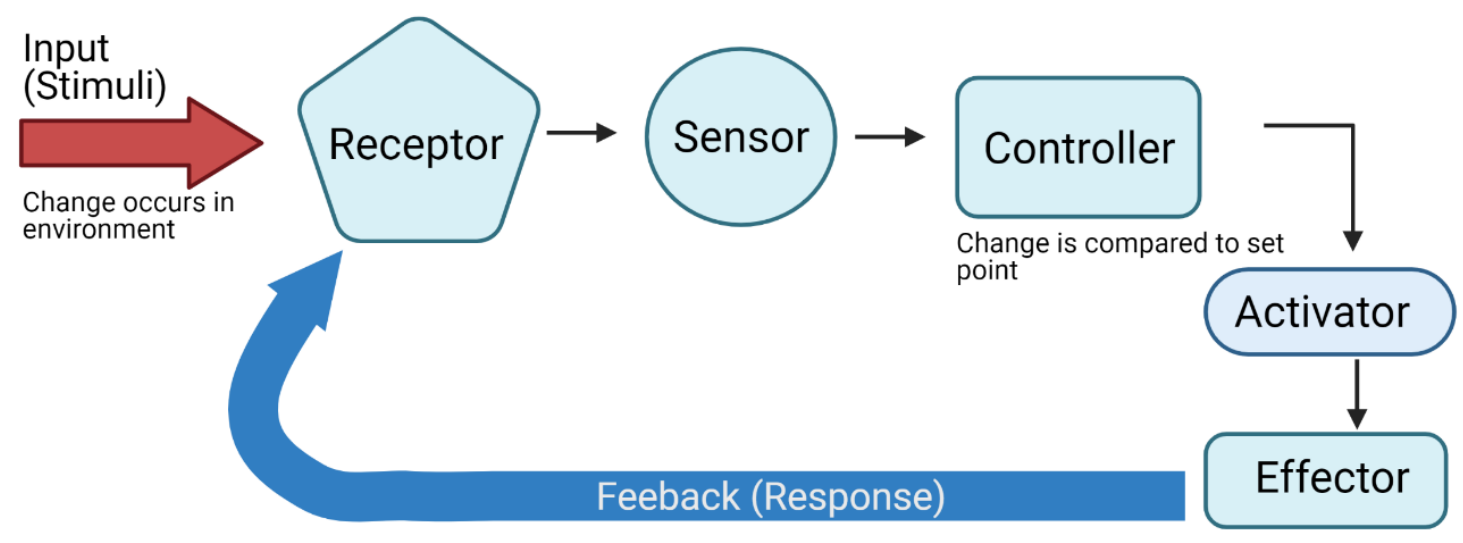

Figure 1. 1. Cellular Response to External Stimuli. When a change occurs in the external environment, cells respond to this input through receptors. Sensors convert this to communicate signals that are meaningful to the organism. A controller compares this signal to a desired "set point" of the homeostatic state to alert the cell if there is a discrepancy. Activators interact with effectos to initiate a response, and this feedback communicates to the original receptor whether or not homeostasis has been achieved. This phenomenon describes how external cues to initiate changes within the cell.

Signal transduction provides instructions for the cell relevant to cell communication, cell cycle control, pathogen sensing, neurotransmission, and many other biological processes that affect target cells within the organism through the initiation of one of four modes of signaling: autocrine signaling, direct signaling, paracrine signaling, and endocrine signaling [3]. Autocrine signaling is a self-activation in which a ligand acts on the same cell that released it, as shown in Figure 1.2.a. Direct cell signaling, as the name suggests, involves direct communication between cells in contact with one another and is often mediated by gap junctions, which are clusters of intercellular channels that allow direct transfer of small molecules and ions. This type of signaling is alternatively referred to as juxtacrine signaling (Fig. 1.2.b). Signaling can also occur between neighboring cells not in direct contact via paracrine signaling. In paracrine signaling, signal ligands diffuse a short distance through the extracellular space to activate the target cell (Fig. 1.2.c). Finally, 
signaling can occur between distant cells. Endocrine signaling is achieved through secreted molecules that travel through the bloodstream to activate target cells that are further away from the signaling cell. This type of signaling is often utilized by hormones (Fig. 1.2.d).

A

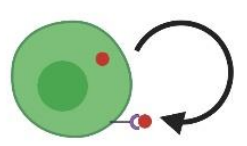

B

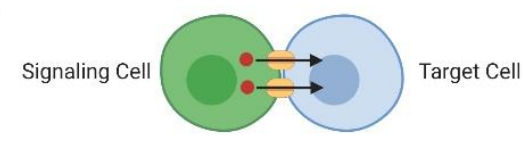

C

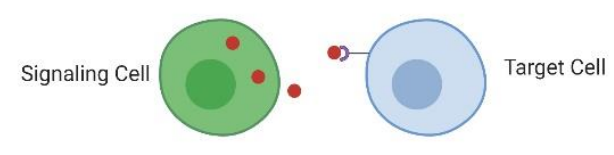

D

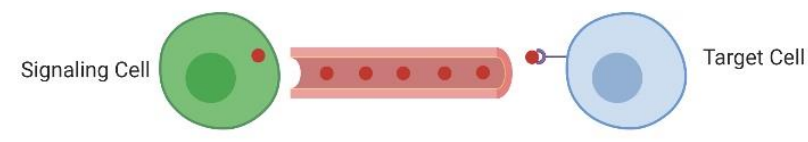

Figure 1. 2. Modes of cellular signal transduction. Signals can be transmitted through four primary mechanisms: (a) autocrine signaling, in which a ligand activates a receptor on the same cell that released it; (b) direct signaling between two cells in contact with one another; (c) paracrine signaling, in which the ligand travels a short distance to bind to a receptor on a target cell; and (d) endocrine signaling occurs when ligands travel through the bloodstream to activate target cells.

Signaling networks can produce immune-related responses through any of these four mechanisms, and sometimes the multiple mechanisms can be employed by the same molecule. Cytokines represent a diverse group of immune-related molecules that participate in these signaling pathways to transmit intracellular and intercellular signals and are an integral part of inflammation and the response to pathogens. The term "cytokine" represents a broad group of 
chemokines, interferons, interleukins, lymphokines, and tumor necrosis factors that play critical roles in cell communication. They typically achieve this through autocrine (Fig. 1.2a) and paracrine (Fig 1.2c) signaling but can simultaneously use multiple signaling mechanisms. Cytokines are often associated with localized signaling to alert and recruit immune cells [4], but many cytokines have illustrated communication via long-range endocrine signaling (Fig. 1.2.d), such as interleukin(IL)-1 transport through the bloodstream to alert distant cells to the presence of pathogens [5].

Once an initiating signal has been produced through any of these mechanisms, it can bind to cell surface receptors. Cell surface receptors traverse the plasma membrane and have specific signal binding domains in the extracellular space, converting external stimuli into an intracellular signal. Upon binding, there is a conformational change to activate the receptor's cytoplasmic domain, often provoking enzyme activity from kinases, phosphatases, and adaptor molecules [6]. Intracellular receptors are present on the nucleus, cytosol, mitochondria, endoplasmic reticulum, and Golgi apparatus and serve to propagate and amplify the signaling events initiated through this extracellular binding. Intracellular binding targets widely vary and frequently affect transcription and gene expression [3], illustrating the connection between external stimuli and intracellular signal transduction pathways, such as activation of the mitogen-activated protein kinase/extracellular signal-regulated kinase (MAPK/ERK) pathway and the Janus kinase/signal transducers and activators of transcription (JAK/STAT) pathway. Extracellular signals (e.g., cytokines) produced in response to stressors, like inflammation and infection, ultimately affect the intracellular pathways that dictate growth, proliferation, survival, and cell death [7]. 
External cues can produce various effects in cells, highlighting the importance of specificity in these signal transduction cascades. Specificity in cell signaling events is a hallmark of ligand binding and allows the cell to be highly adaptive while also producing diverse responses, and this specificity is achieved in two ways. First, receptors exhibit a high affinity for ligands, resulting in specificity of the ligand binding interaction. Receptor specificity depends on the ligand's binding affinity and binding domains, so these specific ligand interactions ultimately dictate the biological effect. Specificity in cytokine binding to members of the JAK/STAT family allows for diverse bioactivity, as is the case with the IL-6 versus IL-10. While both cytokines activate STAT3, IL-6 produces pro-inflammatory effects while IL-10 has an anti-inflammatory role [8]. Another mechanism to achieve specificity in cells is the varied expression of types and proportions of receptors, both inside and outside the cell. Therefore, cell types can be specialized for specific functions depending on the receptors expressed. Immune-related cells express specific and distinct cell surface receptors critical for the host response to pathogens. Antigen-presenting cells (APCs) are essential in the early response to pathogens, and they respond to environmental stimuli through toll-like receptors (TLRs) for pathogen identification [9]. Receptor expression allows diversity of signaling events related to cell type.

Signal transduction networks in the immune response to infection involve an extraordinarily complex system of cascades, and actions of these signaling mediators have consequences for cellular fate. The binding of extracellular signals affects intracellular signaling and produces bioactivity that directs the cell towards survival or death. Specificity of cell signaling is crucial because it allows cells to perceive a wide range of stimuli while maintaining strictly 
regulated biological outputs. Ultimately, these tightly regulated networks enable external cues and stressors to determine cell fate.

\subsubsection{Interactions of cell signaling pathways}

While specific ligand-receptor interactions and varied receptor expression allow for a high degree of specificity, interactions between signaling pathways add another layer of complexity, involving mutual influences of signals originating from disparate pathways. Following the initial signal relay through receptor binding, activation of small molecule second messengers like cAMP, calcium, and DAG can promote second messenger interactions with other pathways. The extent of pathway interactions during immune and inflammatory responses is unknown, but evidence suggests it occurs between different inflammatory cell types, immune-related cytokines, and in intracellular signaling pathways like MAPK/ERK and JAK/STAT [10]. Overlap and integration of cell signaling pathways primarily occur in three different ways: (1) multiple inputs that converge to produce a response, (2) signal gating, in which a signal output from one pathway is regulated by a second pathway, and (3) the establishment of feedback loops. All three of these regulating mechanisms have been observed in signaling networks related to the immune response to infection. These types of interactions between cell signaling pathways highlight the functional specificity of signaling mediators, a term to describe how structurally similar proteins can produce distinct outcomes, introducing more diversity in response.

Coincidence detection is the convergence of two cellular pathways to produce one output; the important distinction from single pathway signaling is that both inputs must be present to elicit a response, and the combined response is different than the individual products of each activation 
(Figure 1.3a). $\mathrm{T}$ cell survival is dependent on $\mathrm{T}$ cell antigen receptor signaling convergence with IL-2 and IL-7 cytokine activation, and, without the presence of IL-2 or IL-7, pro-apoptotic proteins FasL and Bim are up-regulated, leading to apoptosis of T cells [11]. Coincidence detection of these cytokines in conjunction with $\mathrm{T}$ cell antigen receptor signals is critical for $\mathrm{T}$ cell survival. Many other examples exist in complex, highly conserved signaling pathways related to immunity, and these add diversity to the roles of signaling molecules. In response to different cytokines, JAK/STAT pathways can be activated in immune cells to produce distinct, cell-type-specific responses [12]. While many of these signaling events are not well defined, the dependence on multiple pathway convergence does enlighten the diversity of cell signaling outputs.

Gating is another common form of pathway interaction in which one signaling pathway evokes a response and is modulated by a second pathway, resulting in either activation or inhibition of the first pathway (Fig. 1.3b). An important feature of regulation via gating is that one signaling pathway can regulate the flow of another pathway, thus stimulating or prohibiting its response [13]. The GTP binding protein Ras influences the ERK proliferative response through this mechanism via the second messenger cAMP. When cAMP levels increase, protein kinase A (PKA) is activated, which subsequently phosphorylates and deactivates Raf, leading to decreased ERK stimulation and reduced proliferation [14]. The gating mechanism creates a complex web of regulation to connect related cellular processes, ultimately allowing extracellular ligand action from one pathway to dictate intracellular actions related to growth and proliferation. 
Feedback loops establish a direct relationship between cellular outputs and the initial input, and they are influential in signaling networks in homeostasis [15], metabolism [16], transcriptional regulation [17], and immune response to infection [18]. Negative feedback loops are a hallmark of almost all known signaling pathways, attenuating the allowed output through positive or negative feedback. The output signal is produced and converted before being fed back into the input, and this new input can subsequently activate or deactivate the initial pathway (Fig. 1.3c). This type of feedback is necessary for adaptation to the extracellular environment, as eukaryotic cell machinery is built to respond to changes, and cytokine-mediated inflammation employs feedback loops to regulate the degree of inflammation in response to infection or insult. In response to pathogens, IL-1 $\beta$ is released as an early initiator of infection, and subsequent IL- $1 \beta$ signaling elevates levels of its receptor, IL-1Ra, which- in turn- negatively regulates the production of IL- $1 \beta$ and allows a controlled reduction of the immune response [10]. In this way, the cells respond adaptively to changes in their environment rather than solely relying on absolute amounts of a particular signal. Positive feedback occurs when the output is fed back into the input unchanged, thereby amplifying the signal, and this feedforward regulation is also common in immune signaling [19]. In complex networks, signaling pathways can have multiple feedback loops and mixed loops, which contain both positive and negative components. 
Interactions of cell signaling pathways add complexity and allow more variety in biological outputs. Coincidence detection, gating, and feedback loops illustrate three mechanisms by which cells can diversify the responses based on the presence of two or more signaling molecules, which may partially explain the benefit of the pleiotropic and redundant nature of signaling molecules (e.g., cytokines) and how this relates to functional specificity [8]. Gaining insights into the interactions of disparate immune-related signaling pathways will certainly enlighten understanding of the correlations between immune signals and responses when multiple cytokines and immune-related mediators are involved.
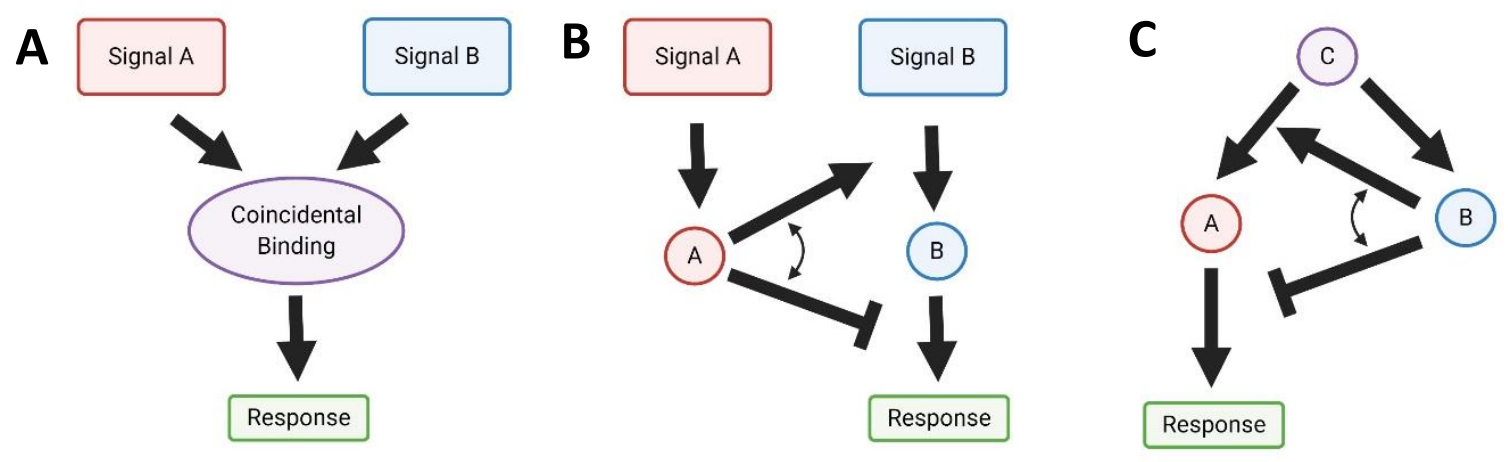

Figure 1. 3. Interactions of Cell Signaling Pathways. Overlap of cell signaling pathways can produce a response in different ways. A) Coincidental detection involves two signals converging to produce one response. B) Gating is a mechanism in which one signal pathway (Signal A) can stimulate or inhibit another pathway (Signal B). C) Feedback loops allow a signal (Signal C) to activate a pathway (Signal A) while also providing regulation of that pathway (Signal B).

\subsubsection{Protein phosphorylation in cell signaling}

Post-translational modifications (PTMs) are a frequently observed mechanism for regulating protein activity in cell signaling cascades, primarily via small covalent changes to their chemical structures (Figure 1.4). PTMs add diversity to the proteome and can modify protein activity through the addition of complex molecules, peptides, chemical groups, and cleavage of 
functional subunits [20]. The addition of these groups often acts as an on/off switch for protein activity, and PTMs serve as regulatory mechanisms for many signal transduction pathways, often through previously-discussed regulatory mechanisms like feedback loops (Fig. 1.3). Protein modifications can also regulate activity via gating by inducing conformational changes that lead to active or inactive states, which can promote or inhibit binding to receptors.

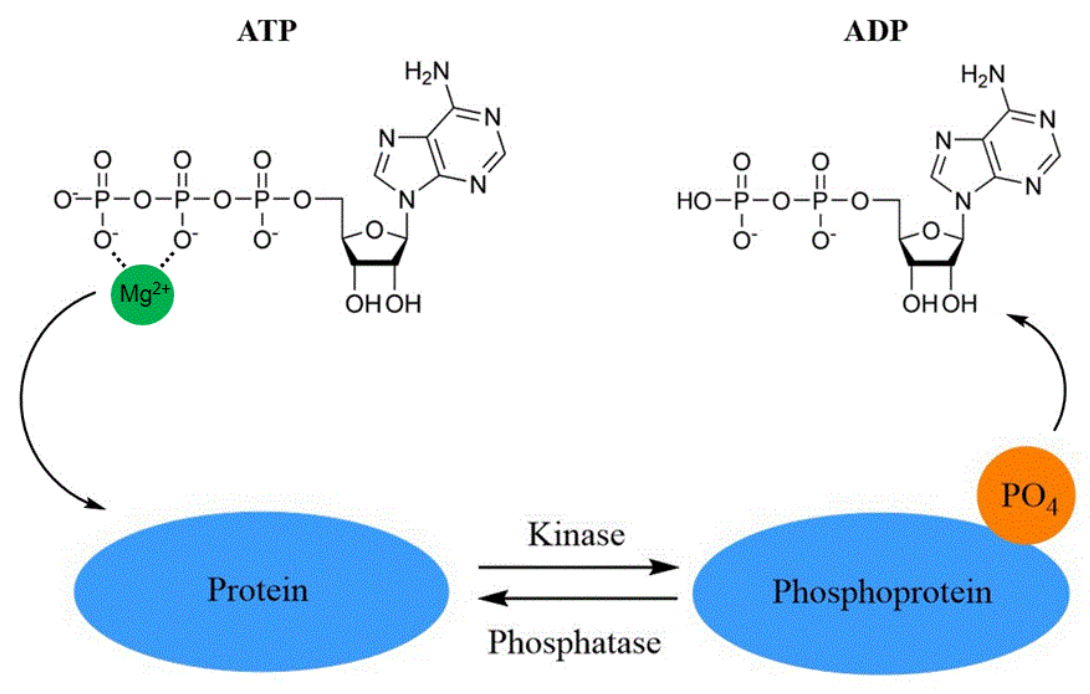

Figure 1. 4. Mechanism of phosphorylation and dephosphorylation. Kinases perform a hydrolysis reaction to covalently attach a phosphate group from ATP to a protein at polar R groups of amino acids. ATP coordination with $\mathrm{Mg}^{2+}$ (shown in green) is essential for the transfer of a phosphate group. Phosphorylated proteins can be dephosphorylated by phosphatases, which hydrolyze the phosphoric acid monoesters, which results in ADP converting back to ATP. A free hydroxyl group remains on the protein after removal of the phosphate group. Reproduced with permission from John Wiley \& Sons, Inc. 
Protein phosphorylation was one of the first PTMs identified, and it has a role of regulation in key biological processes through simple biding of a phosphate group. In 1956, Krebs and Fischer demonstrated that reversible phosphorylation controlled glucose storage [21]. Through kinase activity, phosphate groups are added to polar R groups of serine, threonine, or tyrosine residues.

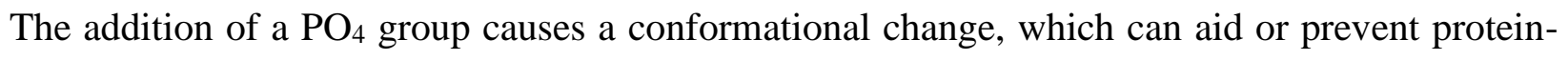
protein interactions. Phosphorylation is negatively regulated by phosphatase enzymes, which can remove the phosphate group, and the mechanism of phosphorylation/dephosphorylation is illustrated in Figure 1.4 [22]. These processes influence energy availability, making phosphorylation an especially important PTM in cellular signaling. Energy availability is predictive of cellular fate, and this can be experimentally observed by monitoring protein phosphorylation as a marker of mitochondrially-driven kinase activity [23]. Phosphorylation has been studied in bacterial and mammalian systems and has demonstrated a role in a broad range of cellular processes, such as membrane transport, protein degradation, and enzyme regulation. The biological implications of these activities are important in bioenergetics, cell proliferation, and the development of disease states. Monitoring phosphorylation events, especially concerning immune signaling, has proven useful in understanding the disrupted cell signaling events that can delay healing [24].

Historically, cell signaling research has focused on distinct pieces of signaling cascades to understand specific regulators, but spatial and temporal regulation of these signals, including PTMs, is an increasingly compelling problem to study in the field of biochemistry. New developments in multi-'omics technologies have made the investigation of a large number of related targets more accessible than ever before. Recent research efforts have focused on 
understanding not only the individual components of cell signaling pathways, but they have also expanded to investigate interactions between pathways. Many researchers have begun to approach this problem by studying signaling pathways as a whole to understand the influence of individual components and their respective relationships. Future research can continue to understand how these complex signaling pathways interact to produce diverse biological consequences.

\subsection{The immune response to infection}

\subsubsection{Acute immune response}

The mammalian immune system serves to protect the host from harmful pathogens, and this response involves the coordination of multiple cell signaling pathways, including cytokine immune mediation [25], MAPK/ERK activation [26], and JAK/STAT signaling [27]. The immune system's primary function is to eliminate pathogenic microbes if they breach the mucosal barrier, and both innate and adaptive immune strategies are utilized to identify and target harmful pathogens via pathogen recognition receptors (PRRs). PRRs recognize specific structures of pathogens known as pathogen-associated molecular patterns (PAMPs), and this initiates a cascade to eliminate the pathogen [28]. The acute immune response is the initial attempt to combat the infection. Cytokines are essential mediators of this response, acting as communicators to recruit immune cells and resolve infection through short-range autocrine signaling and paracrine signaling [4, 5], and long-range endocrine signaling [29]. Cytokines are produced by immune-specific cells such as B lymphocytes, T lymphocytes, macrophages, and mast cells [30], or by endothelial cells, fibroblasts, and stromal cells [31], which are all capable of releasing cytokines as an "alert" to the localized environment. Murine models have established that this acute response period commences at pathogen recognition and can persist to approximately 21 days post-infection [32]. The initial 
inflammatory response activates NF- $\mathrm{kB}$ and MAPK pathways, resulting in the production of cytokines IL-1 $\alpha$, IL-1 $\beta$, TNF- $\alpha$, IL-2, and IL-18 [33]. These cytokines regulate the activities of Thelper cells, macrophages, and neutrophils to control immune cell differentiation and maturation [34], interact with growth factors that control angiogenesis [35], and influence cellular fate by activation of NF-kB signaling to regulate apoptosis [33]. Extracellular cytokines bind to receptors to propagate these intracellular signaling cascades, impacting the transcription of proteins central to these cellular processes. Cytokines like IL-6, IL-10, IL-12, IL-4, IL-13, and IFN- $\alpha / \beta$ activate STAT proteins, activating TH1-related responses to propagate immune cell activity [27]. The effects of early cytokine activity produce a robust inflammatory response that is beneficial for resolving pathogens, but it is somewhat unregulated.

The potent inflammatory response elicited early on by cytokines can combat bacterial invasion, but it can also result in tissue damage if left unregulated. The initial cytokine response functions much like a sensor of infection, followed by a more targeted approach adopted by the immune system following this uncontrolled release of cytokines, with careful control of activity through gating, feedback mechanisms, and signaling crosstalk. The downstream activity of cytokine activation through receptor binding often serves as a negative regulator of cytokine activity [10], so cells can adapt following the initial cytokine surge. Sophisticated communication between multiple cell signaling pathways, including MAPK/ERK, NF-KB, and JAK/STAT signaling [29] to balance the activity of pro-inflammatory and anti-inflammatory cytokines facilitate this adaptation to resolve infection most efficiently. The cytokine response to infection persists until the infection has been eliminated from the host. Balancing the initial inflammatory 
response and activation of effectors achieves pathogen resolution without producing harmful effects in surrounding tissues.

\subsubsection{Chronic infections and immune response}

When the acute response to infection cannot resolve or fully eliminate the pathogen, the infection can transition into a chronic state, and both environmental and biological factors can contribute to this outcome. The causes for failure of acute response are not fully understood, but several risk factors have been identified, including autoimmune disease, obesity, and age [36]. During infection, a critical balance must be struck by immune mediators to prevent tissue damage, but chronic infections result in persistent activation of inflammation until immune cells become exhausted [37], causing a variety of consequences for cell signaling. In chronic bacterial infections, disrupted immune responses are marked by continuous stimulation of Th1/Th17 lymphocyte proinflammatory mediators and a failure of Th2 anti-inflammatory mediators [38]. Reduction of antiinflammatory influence causes the signaling networks to be shifted towards excessive cell death, which disrupts immune cells' ability to resolve the infection and damages the surrounding tissue, thereby perpetuating the infection. Network analysis of chronic infection states has revealed distinct shifts away from growth, proliferative, and immune differentiation metabolic activities hallmark of the acute response towards heavy metabolic activity with increased progression towards apoptosis and programmed cell death [39].

Errant cell signaling has also been attributed to failures in signal transduction regulatory loops. In chronic infections, the deactivation of crucial downstream regulators of cytokine activity 
has been observed, such as dephosphorylation in c-Jun, p38, and ERK1/2 [40]. Phosphorylation of these proteins typically serve as negative regulators of inflammatory cytokine activity, but this represents one example of disrupted cell signaling cascades in chronic infection states. While this has not been explicitly linked to the disrupted cytokine activity and reduced anti-inflammatory capacity, it introduces a potential explanation for how a shift in the cytokine pro- and antiinflammatory balance may eventually stimulate increased cell death [41]. The reduced capacity of immune cells observed in persistent infection suggests that these signaling disruptions overwhelm host immunity, causing an inability to resolve the infection. Disruptions to signaling networks can be influenced by infection duration, infecting organism, and tissue type.

\subsubsection{Bacterial hijacking of host cell signaling}

While the mechanisms of disrupted cell signaling in infection are not entirely understood, microbial agents have demonstrated the ability to hijack host cell signaling machinery to decrease the efficacy of host defense strategies, and some of these effects play a role in the transition to chronic infection. One way bacteria affect host immune response is by producing toxins that corrupt cell signaling cascades and dampen the effects of mediators critical for the early recognition of pathogens [42]. Without this initial alert and warning to the host immune system, pathogens can go undetected, ultimately disrupting the normal sequence of pathogen clearance via immune signaling [43]. The VacA cytotoxin of $H$. pylori has been shown to delete genes in host immune cells vital to inflammatory response, blocking several early pro-inflammatory cytokines involved in early infection response like IL-6 and IL-8 [44]. Similarly, M. tuberculosis subverts macrophages to downregulate TNF- $\alpha$, IL-12, and IL-1 $\beta$ and shut down pro-inflammatory responses [45]. Bacteria like Yersinia ssp., Shigella ssp., and E. coli produce other peptides or 
small molecules that can interfere with cell signaling cascades by inhibiting critical signaling mediators and coopt entire signaling systems, resulting in a decreased ability for pathogen recognition [46]. These bacteria cause deleterious effects through the exploitation of host signaling.

Bacterial hijacking of cell machinery has been observed for many pathogens, and the exact mechanisms of disruption vary depending on infecting organism and the extracellular environment. The presence of non-infectious inflammatory stimuli, such as those associated with obesity or chronic inflammatory conditions, can influence how bacteria interact with host cell signaling pathways. The unique environment created by inflammatory disease coupled to infection is more complex than bacterial invasion alone because the cascades initiated by recognition of PAMPs often overlap with those triggered by damage-associated molecular patterns (DAMPs) from noninfectious stimuli. Both PAMPs and DAMPs cause persistent activation of pro-inflammatory mediators through activation of NF- $\mathrm{BB}$ and p38 MAPK pathways [47], so the overlap of chronic inflammatory disease and infection may not have the same effect as the sum of the individual insults. The intersection of chronic inflammatory conditions and subsequent infection is not well studied, but obesity and rheumatoid arthritis are two conditions that are frequently plagued by concurrent infection. Obesity impairs the immune response to infection by promoting $\mathrm{T}$ cell senescence, which affects the production of early pro-inflammatory cytokines [48]. Although the mechanistic effects have not been explicitly studied, increased incidence of chronic infection in obese patients compared to non-obese individuals suggest the suppressive effects on cell signaling may be advantageous for bacterial interference with signaling cascades. Rheumatoid arthritis is another disease that alters immune-related cell signaling and has been associated with an increased risk of chronic infection. Several cytokines, like $1 \mathrm{~L}-1 \alpha$, IL-1 $\beta$, and TNF- $\alpha$, are up-regulated in 
arthritis, and chronic activation of these pro-inflammatory mediators can cause damage to bone and cartilage [49]. Damaged tissue creates an ideal environment for infection, so the compromised tissue combined with immunomodulatory effects of the disease may create an environment that is conducive to bacterial disruption of immune function. Altered immune signaling when both infectious and non-infectious sources are present continues to be an intriguing problem, and the rising prevalence of chronic inflammatory conditions like obesity and rheumatoid arthritis in the United States makes them an extremely relevant problem to study [50].

\subsection{Disruptions to cell signaling pathways observed in pathological conditions}

\subsubsection{Periprosthetic joint infection (PJI) and immune signaling}

Elucidating changes in cell signaling networks provoked by chronic infection may provide a better understanding of the drivers of dysregulation in a range of clinical pathologies. One example of a chronic infection is periprosthetic joint infection (PJI), a devastating post-operative complication following total knee arthroplasty (TKA) and total knee revision (TKR) procedures, and these infections have high clinical relevance for the field of Orthopaedics. Over one million joint revision procedures are conducted in the United States every year, and the popularity of TKA procedures is projected to grow rapidly [51]. While joint replacement is a life-enhancing procedure for many people, infections can develop and affect both the joint prosthesis and surrounding tissues. PJIs occur in approximately $2 \%$ of joint revisions and affect tens of thousands of patients per year, imposing high emotional and financial burdens on these individuals $[52,53]$. They can be caused by a variety of bacteria, but Staphylococcus aureus is the most common pathogen implicated [54]. Many PJIs become chronic due to bacteria's ability to evade host response by forming protective 
biofilm barriers and interference with host immune efforts [55], and changes in cytokine and immune signaling pathways have been observed.

The localized PJI environment is affected both by inflammation due to foreign body implants and the modulatory effects of the bacteria. Surrounding tissues often exhibit elevated levels of localized cytokines due to prosthesis alone [56], and the presence of S. aureus can have additional effects on host adaptive immunity. PJIs due to $S$. aureus have been shown to dampen leukocyte activity and reduce phagocytosis following bacterial invasion. S. aureus also employs countermeasures, including provocation of anti-inflammatory mediators, to combat the robust proinflammatory response of surrounding tissues [57], similar to previously discussed bacterial hijacking strategies to target early pathogen recognition. The prevailing theory of why PJIs become chronic and difficult to treat is the devastation to the localized environment overcomes the immune system's ability to combat the infection, so it is critical to dissect the specific influences of PJIs on surrounding tissues. The effects of chronic PJI on localized tissues have recently become of interest for researchers in the field of Orthopaedics [58-60].

Studying the specific deviations in immune signaling could lead to the identification of tissue-level biomarkers of PJI relevant to treatment. Once chronic PJI is established, it is difficult to treat, but surgical strategies can manage the infection. The gold standard involves debridement and irrigation of the wound area to remove infected tissues, either decreasing the bacterial load to a level the immune system can overcome or completely removing the infected tissues. While this strategy shows the highest rates of success in persistent PJI, it is expensive and often requires 
additional surgical intervention [52]. Further, debridement is a subjective technique in which the surgeon is tasked with visually inspecting the tissue to determine viability. While Orthopaedic surgeons undergo extensive training, it can be challenging to eradicate infected tissue using only visual inspection. Even a small number of bacteria remaining (i.e., a few colonies) can lead to reinfection of the joint [61]. Failure rates of procedures can reach as high as 50\% in infected joints, partly because subjective measures of debridement are not sufficient [62]. Delineating healthy and infected tissues in PJI is critical, but no tissue-level biomarkers are established for intra-operative assessment to inform treatment.

Several biomarkers have been identified for their diagnostic utility in PJI but have not been investigated for their ability to identify healthy versus infected tissues. While serum and synovial fluid measurements of cytokines like IL-1 $\beta$, IL-6, IL-10, and IL-8 have been incorporated into clinical protocols for PJI diagnosis [63], they do not provide information about tissue viability. Establishing diagnostic biomarkers of infected tissues in PJI requires a high degree of sensitivity and specificity [64]. Sensitivity is a measure of how well the biomarker identifies true positives (e.g., how many tissues are correctly identified as infected; Equation 1.1). Low sensitivity can lead to a high number of false negatives, or type II error. Specificity describes how well the biomarker determines true negatives (e.g., how many healthy tissues can be identified as non-infected; Equation 1.2). Low specificity can lead to a high number of false positives, or type I error. In biomarker development, there is a balance between sensitivity and specificity, with higher sensitivity leading to lower specificity and vice versa. 


$$
\begin{aligned}
& \text { Sensitivity }=\frac{\text { True Positives }}{\text { True Positives }+ \text { False Negatives }} \\
& \text { Specificity }=\frac{\text { True Negatives }}{\text { True Negatives }+ \text { False Positives }}
\end{aligned}
$$

Biomarker analysis through enzyme-linked immunosorbent assays (ELISAs) is an attractive analytical technique to investigate targets related to tissue health in PJI. Due to the complexity of the inflammatory environment, it would likely be advantageous to study a suite of immune-related targets and the healing cascades associated with tissue damage in chronic infection and inflammation. Many immune and healing mediators exhibit low homeostatic concentrations in blood or localized fluids (e.g., picomolar) [65, 66], but stimulation by inflammatory stressors like infection can cause increases of up to 1,000-fold [67]. Cost-effective, high throughput multiplexed ELISAs offer a solution to investigate multiple targets related to immune and healing processes simultaneously. Multiplexed assays use multiple antibodies immobilized to the surface of a polystyrene bead, and measurements are made using a dual-laser flow cytometric system [68]. Multiple detection antibodies are used, and dyes correspond to the analyte of interest, and up to 100 different antibody-antigen combinations can be included per bead [69]. A classification laser identifies the unique signature of each analyte region, and a reporter laser measures the fluorescence intensity of the signal (Figure 1.5). The accuracy of this method is dependent on CV, upper and lower limits of quantitation [70], and quality of the calibration curve but has shown analytical performance comparable to a singleplex ELISA [71]. 

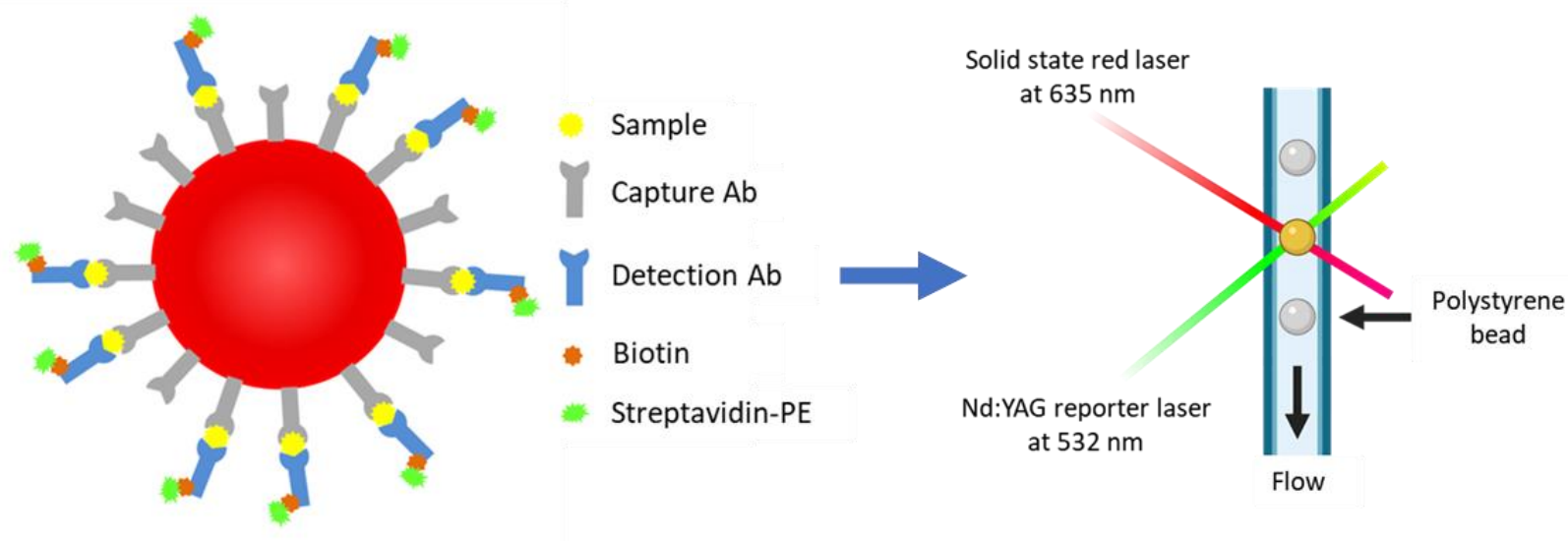

Figure 1. 5. Bead-based ELISA. Microsphere beads have multiple antibodies immobilized to the surface, corresponding to different antigens. A two-laser system consists of: (1) one detection to measure emission of dye specific to each analyte, and (2) another detection to measure the emission of Streptavidin-PE (or similar fluorophore) to measure the fluorescence intensity and quantify each analyte. The multiplexed assay format proceeds similar to a traditional sandwich ELISA, but many capture antibodies are immobilized on the surface. The biotinylated detection antibody shown in blue recognizes a separate epitope of your desired analyte. The biotinylated detection antibody then binds with the fluorescent reporter, streptavidin-phycoerythrin (shown in green), due to the extremely high binding affinity of biotin for streptavidin. This image was modified with permission from Vrana 2015 [72].

\subsubsection{Dysregulation of cell signaling in other conditions}

Disrupted cell signaling processes are common in many other conditions, such as autoimmune disorders, cancer, and Alzheimer's Disease, and investigating signaling networks has led to advances in diagnostics and therapeutics for these pathologies. Here, a few examples are discussed to highlight the potential for similar research to provide insight for chronic infections like PJI. 
Cytokines IL-4 and IL-13 are potent initiators of allergic response, and the IL-4/IL13/STAT6 pathway is central to asthma modulating [73]. Elevation of these factors has been observed in inflammatory airways of asthmatics, and some asthma drugs target IL-4/IL-13/STAT6 to suppress the inflammatory response. Targeting cell signaling pathways in asthma has not only led to better drug targets, but differential network analysis has also revealed disparate hub genes associated with inflammation, apoptosis, and T cell activity for allergic responses [74]. Networks associated with various cancers have also been probed to identify new therapeutic targets. Many cancers involve disrupted cell signaling, especially processes related to proliferation and survival. Novel therapies for hepatocellular carcinoma have been reached through targeting of Ras/Raf/MAPK [75], PI3K/AKT/mTOR [76], Wnt/ß-catenin [77], and hedgehog signaling pathways [78], four highly evolutionarily conserved pathways relevant to a number of critical cellular processes. Recently, studies of Alzheimer's Disease illustrated that new immune-related targets HLA-B, IL-10, C1QB, and CD86 could be pursued to prevent disease and that disease etiology of Alzheimer's showed similarities to antigen presentation through network analysis [79]. Dissecting the complex signaling pathways in these conditions provided a better understanding of the alterations to normal cell signaling provoked by disease states. These represent a few of the many examples to illustrate the utility of investigating signal transduction networks in disease.

\subsection{Bioinformatics approaches to understand complex biological responses}

Biomarkers for diagnosis and treatment have been elucidated for many diseases, from infection to autoimmune inflammatory disorders to cancer and beyond, and researchers employ different statistical approaches and methodologies to understand these complex responses. It is often advantageous to approach these using network analysis rather than traditional statistical 
testing like t-tests and ANOVAs, and studying chronic infection like PJI through network analysis may provide a better understanding of the complex inflammatory environment. Network analysis approaches can involve the integration of different data types and allow researchers to investigate interactions of different targets involved (i.e., transcripts, proteins, metabolites) [80]. These approaches have expanded on classical statistical techniques to probe beyond the investigation of a single molecular entity. Recent accessibility of multi-'omics technologies have made networkwide assessments more common when studying disease states, and new multi-'omics studies of disease have led to the discovery of biomarkers with higher specificity than those identified in classical statistical testing (e.g., ANOVA, t-test, ROC), and this may be due to the ability to consider the entire network as a whole rather than analyzing each target in isolation [81]. Database searching software applications and mathematical modeling techniques have been utilized in a variety of diseases to understand underlying molecular patterns of disease etiology.

\subsubsection{Background on common network methodologies}

Biological network analysis is central to understanding complex biological processes, and recent technologies have shifted research towards large-scale biological datasets that measure many parameters. Multi-'omics studies (e.g., transcriptomics, genomics, proteomics, metabolomics, interactomics) are the primary ways researchers approach network studies, covering every portion of the journey from DNA to protein to protein-protein interactions in signaling [82-84]. Ultimately, studying biological responses through these various platforms allows a holistic view of cells and tissues to identify which targets are most important for survival or death. High-throughput technologies like next-generation sequencing, microarrays, and hybrid screening to identify interactions of biological network components have made it possible to 
collect a large amount of data with relative ease and allowed exponential growth of network repositories and databases to aid in the analysis of cellular network data. Cutting-edge mass spectrometry techniques allow for global assessment of proteins, metabolites, and other species of interest in biological samples [85]. The advent of multi-'omics approaches has paved an exciting path forward, allowing for a more complete understanding of complex biological interactions. These technologies can be applied for biomarker identification [86], drug discovery [87], and in the future of personalized medicine [88].

Researchers that take advantage of these techniques produce hearty data sets with large amounts of information, but this presents a problem of its own: what is the best way to analyze, integrate, and interpret the data? Biological networks and interactions of proteins within the network can be constructed from literature-derived and experimental data. Additionally, data can be acquired in many forms, including protein-protein interactions, protein function prediction, association with canonical networks, and more. Some commonly-used network methodologies are based on graph theory, Bayesian approaches, and correlations to draw connections between individual targets to form a network [81]. These approaches can be used to answer a wide range of biological questions concerning genetic causes of disease, target identification for biomarkers of drug discovery, and monitoring of treatment [89].

\subsubsection{Databases and model repositories}

Databases and repositories are commonly used in biological network analysis, and they often take both literature-derived data and the researcher's experimental data into account. Many databases exist primarily to analyze network data, and the researcher must consider the nature of 
the data, application, and interpretation when deciding which database(s) to utilize. Model organisms, tissue types, and diseases may also be considered; databases and model repositories are carefully curated, but some may be more appropriate for different etiologies [90]. These network-based studies are often carried out in model organisms, including both in vitro and in vivo research. These are considerations when choosing which database(s) to use and what caveats must be acknowledged when interpreting the data. Some examples include the Reactome Pathway Knowledge Base [91], KEGG genome database [92], Gene Ontology (GO) GOnet [93], Ingenuity Pathway Analysis (IPA) [94], and Cytoscape [95]. Most of these tools rely on basic statistical tests to identify significant targets. Reactome uses a simple binomial test to calculate significant deviations from expected observations and create links between different entities. KEGG and GO use hypergeometric probabilities to enrich data sets by identifying associations with individual nodes of interest. IPA connects individual targets through Fisher's Exact Test to construct a network of biological nodes. Some tools, like Cytoscape, allow the user to have more control over the tests for significance, which provides more flexibility when working with different types of data. Databases differ in analysis methods, so researchers must thoroughly understand what information the database can provide and its limitations. Some common databases and repositories used to interpret biological networks are discussed in the following paragraphs.

Systems biology approaches have centered around network analysis, and these techniques have demonstrated broad utility in addressing questions of disease etiology to therapeutics, making them extremely attractive to researchers. Studies cover a wide range of data types, collection methods, statistical analysis approaches, and aims, demonstrating the highly diverse studies that benefit from network analysis techniques. Network analysis using databases and repositories have 
led to better targets for cancer therapeutics [96], a better understanding of the immune response to asthma [97], and biomarkers of disease progression in Amyotrophic Lateral Sclerosis (ALS) [98], to name a few of the many pathological states that have been investigated. While it is often advantageous to use network approaches, problems can arise due to insufficiencies in data related to a particular model organism, the number of relevant studies in the database for a specific disease, and lack of spatial and temporal information (i.e., mismatches in time points or distance from injury) [89]. Progress in data analysis in the future will depend on broadening the model organisms and scopes of disease, including the inclusion of multiple 'omics platforms for diseases. The more data within the repositories these databases use, the more accurate the machine learning algorithms can become, ultimately providing better answers to systems biology questions. Network approaches have expanded the knowledge of biological systems and have proven to be an essential asset for the future of signal transduction research.

\subsubsection{Advanced mathematical network analysis approaches}

The use of biological network applications to analyze a dataset is highly beneficial for many researchers, especially in disease states that have been well studied. Challenges can arise when investigating diseases that have not been well characterized and only have a small number of studies relevant to signal transduction mediators. For these cases, advanced statistical techniques like mathematical modeling offer an alternative, as they rely solely on the parameters the investigator includes. Network centrality is one approach, and it is based upon graph theory, which mathematically organizes the different parameters to create a "map" of their interactions and interconnectivity [99]. In graph theoretical analysis, the network consists of nodes and edges. Nodes are parameters within the network (i.e., proteins, genes, metabolites), and edges represent 
the interactions of node pairs. Using graph theory, researchers can characterize the biological network by connecting nodes and assigning quantitative values to their influence on the network as a whole. A common approach for interpretation of these networks is to analyze through a lens of network centrality, as this can properly weight the interactions of the network, providing information on highly interconnected nodes vs. peripheral nodes, which can then be interpreted for their biological roles [100].

Many other advanced mathematical modeling and statistical techniques have been employed to analyze networks, including principal component analysis (PCA), partial least squares discriminant analysis (PLS-DA), and hierarchical clustering [101]. These techniques reduce the dimensions of the dataset and combine parameters to model the system, thereby significantly reducing the complexity and allowing interpretation. Predictive modeling can be integrated into these techniques to evaluate the model's ability to accurately group proteins, genes, etc., to describe an altered biological state. New tools that utilize advanced statistical principles to dissect large biological network data are being developed in the field, and many of them are based upon these techniques. For example, new statistical strategies have been employed to integrate multi'omics data based on orthogonal PLS approaches, illustrating the potential to narrow variables of interest [102]. The probabilistic modeling technique ProbRules was developed to predict the behavior of dynamic signaling networks based on differential equations, allowing the ability to focus wet-lab experiments a priori [103]. Williams et al. developed a "functional heatmap" to quickly assess time-series multi-'omics data based on cluster analysis, providing the means to quickly assess patterns in large data sets [104]. These represent only a few examples of the possibilities of new tools developed to support signal transduction research. At their cores, 
traditional multivariate statistical approaches laid the foundation for these tools and allowed researchers to expand their use to handle multi-'omics data sets.

Choosing which mathematical technique is appropriate for a data set is dependent on the format of the data, desired outcomes, and goals of the research. All of the described analysis methods have been proven in the literature to manage large, complex biological datasets, and researchers are developing new techniques to integrate multi-'omics data sets for their own purposes. As multi-'omics techniques become more widely adopted for studies of biological networks, network analysis through database searching, mathematical modeling, and repositories highlight a new frontier to dissect complex biological milieu to understand diseases like chronic infection.

The work presented in the following chapters describes comparisons of immune-related cytokines and wound healing phosphoproteins in response to implants and infection in order to understand cell signaling changes in response to these stimuli. Based on previous work to understand cytokine responses in PJI on a serum level $[52,53]$, the prevailing hypothesis was that higher concentrations of cytokines would be present in septic tissues, and these could be developed into tissue-level biomarkers of infection. Phosphoproteins related to wound healing processes were also tested to probe the interactions between inflammatory immune cytokines and tissue healing mediators. The purpose of this work was to provide a novel tissue-level investigation to provide new insights into changes related to implants and chronic infection. 


\subsection{References}

[1] Kvetnoy IM, Reiter RJ, Khavinson VK: Letter to the Editor. Claude Bernard was right: hormones may be produced by "non-endocrine" cells. Neuro Endocrinol Lett 2000, 21:173-174.

[2] Hildebrand E: What does Halobacterium tell us about photoreception? Biophys Struct Mech 1977, 3:69-77.

[3] Cooper G: Signaling Molecules and Their Receptors. In The Cell: A Molecular Approach. Second Edition edition. Sunderland (MA): Sinauer Associates; 2000

[4] Xue Q, Lu Y, Eisele MR, Sulistijo ES, Khan N, Fan R, Miller-Jensen K: Analysis of single-cell cytokine secretion reveals a role for paracrine signaling in coordinating macrophage responses to TLR4 stimulation. Sci Signal 2015, 8:ra59.

[5] Imura H, Fukata J, Mori T: Cytokines and endocrine function: an interaction between the immune and neuroendocrine systems. Clin Endocrinol (Oxf) 1991, 35:107-115.

[6] Li S: Mechanisms of cellular signal transduction. Int J Biol Sci 2005, 1:152.

[7] Plotnikov A, Zehorai E, Procaccia S, Seger R: The MAPK cascades: signaling components, nuclear roles and mechanisms of nuclear translocation. Biochim Biophys Acta 2011, 1813:1619-1633.

[8] Moraga I, Spangler J, Mendoza JL, Garcia KC: Multifarious determinants of cytokine receptor signaling specificity. Adv Immunol 2014, 121:1-39.

[9] Zhu Y, Yao S, Chen L: Cell surface signaling molecules in the control of immune responses: a tide model. Immunity 2011, 34:466-478. 
[10] Schmitz ML, Weber A, Roxlau T, Gaestel M, Kracht M: Signal integration, crosstalk mechanisms and networks in the function of inflammatory cytokines. Biochim Biophys Acta 2011, 1813:2165-2175.

[11] Riou C, Yassine-Diab B, Van grevenynghe J, Somogyi R, Greller LD, Gagnon D, Gimmig S, Wilkinson P, Shi Y, Cameron MJ, et al: Convergence of TCR and cytokine signaling leads to FOXO3a phosphorylation and drives the survival of CD4+ central memory T cells. J Exp Med 2007, 204:79-91.

[12] Bezbradica JS, Medzhitov R: Role of ITAM signaling module in signal integration. Curr Opin Immunol 2012, 24:58-66.

[13] Jordan JD, Iyengar R: Modes of interactions between signaling pathways. Biochem Pharmacol 1998, 55:1347-1352.

[14] Dhillon AS, Pollock C, Steen H, Shaw PE, Mischak H, Kolch W: Cyclic AMP-dependent kinase regulates Raf-1 kinase mainly by phosphorylation of serine 259. Mol Cell Biol 2002, $22: 3237-3246$.

[15] Cannon WB: PHARMACOLOGICAL INJECTIONS AND PHYSIOLOGICAL INFERENCES. Science 1929, 70:500-501.

[16] CHANCE B, ESTABROOK RW, GHOSH A: DAMPED SINUSOIDAL OSCILLATIONS OF CYTOPLASMIC REDUCED PYRIDINE NUCLEOTIDE IN YEAST CELLS. Proc Natl Acad Sci U S A 1964, 51:1244-1251.

[17] MONOD J, JACOB F: Teleonomic mechanisms in cellular metabolism, growth, and differentiation. Cold Spring Harb Symp Quant Biol 1961, 26:389-401. 
[18] Greenhalgh CJ, Hilton DJ: Negative regulation of cytokine signaling. J Leukoc Biol 2001, 70:348-356.

[19] Beutler B: Microbe sensing, positive feedback loops, and the pathogenesis of inflammatory diseases. Immunol Rev 2009, 227:248-263.

[20] Wang YC, Peterson SE, Loring JF: Protein post-translational modifications and regulation of pluripotency in human stem cells. Cell Res 2014, 24:143-160.

[21] KREBS EG, FISCHER EH: The phosphorylase $b$ to a converting enzyme of rabbit skeletal muscle. Biochim Biophys Acta 1956, 20:150-157.

[22] Lee MJ, Yaffe MB: Protein Regulation in Signal Transduction. Cold Spring Harb Perspect Biol 2016, 8.

[23] Vrana JA, Currie HN, Han AA, Boyd J: Forecasting cell death dose-response from early signal transduction responses in vitro. Toxicol Sci 2014, 140:338-351.

[24] Han AA, Currie HN, Loos MS, Vrana JA, Fabyanic EB, Prediger MS, Boyd JW:

Spatiotemporal phosphoprotein distribution and associated cytokine response of a traumatic injury. Cytokine 2016, 79:12-22.

[25] Arango Duque G, Descoteaux A: Macrophage cytokines: involvement in immunity and infectious diseases. Front Immunol 2014, 5:491.

[26] Arthur JS, Ley SC: Mitogen-activated protein kinases in innate immunity. Nat Rev Immunol 2013, 13:679-692.

[27] Scott MJ, Godshall CJ, Cheadle WG: Jaks, STATs, Cytokines, and Sepsis. Clin Diagn Lab Immunol 2002, 9:1153-1159. 
[28] Mogensen TH: Pathogen recognition and inflammatory signaling in innate immune defenses. Clin Microbiol Rev 2009, 22:240-273, Table of Contents.

[29] Altan-Bonnet G, Mukherjee R: Cytokine-mediated communication: a quantitative appraisal of immune complexity. Nat Rev Immunol 2019, 19:205-217.

[30] Dube PH, Revell PA, Chaplin DD, Lorenz RG, Miller VL: A role for IL-1 alpha in inducing pathologic inflammation during bacterial infection. Proc Natl Acad Sci U S A 2001, 98:10880-10885.

[31] JL M-C, JFC C, O G-C, PT V-G, LG R-G, VE H-R: Cytokine profiling plays a crucial role in activating immune system to clear infectious pathogens. In Immune Response Activation and Immunomodulation. InTech Open; 2018

[32] Torrado E, Cooper AM: Cytokines in the balance of protection and pathology during mycobacterial infections. Adv Exp Med Biol 2013, 783:121-140.

[33] Liu T, Zhang L, Joo D, Sun SC: NF- $\mathrm{B}$ signaling in inflammation. Signal Transduct Target Ther 2017, 2.

[34] Xie K, Dai K, Qu X, Yan M: Serum and Synovial Fluid Interleukin-6 for the Diagnosis of Periprosthetic Joint Infection. Sci Rep 2017, 7:1496.

[35] Zhang JM, An J: Cytokines, inflammation, and pain. Int Anesthesiol Clin 2007, 45:27-37.

[36] O'Connor SM, Taylor CE, Hughes JM: Emerging infectious determinants of chronic diseases. Emerg Infect Dis 2006, 12:1051-1057.

[37] Wherry EJ: T cell exhaustion. Nat Immunol 2011, 12:492-499. 
[38] Szkaradkiewicz A, Karpiński TM, Zeidler A, Szkaradkiewicz AK, Masiuk H, GiedrysKalemba S: Cytokine response in patients with chronic infections caused by Staphylococcus aureus strains and diversification of their Agr system classes. Eur J Clin Microbiol Infect Dis 2012, 31:2809-2815.

[39] Doering TA, Crawford A, Angelosanto JM, Paley MA, Ziegler CG, Wherry EJ: Network analysis reveals centrally connected genes and pathways involved in CD8+ T cell exhaustion versus memory. Immunity 2012, 37:1130-1144.

[40] Hashino M, Tachibana M, Nishida T, Hara H, Tsuchiya K, Mitsuyama M, Watanabe K, Shimizu T, Watarai M: Inactivation of the MAPK signaling pathway by Listeria monocytogenes infection promotes trophoblast giant cell death. Front Microbiol 2015, 6:1145.

[41] Saeidi A, Zandi K, Cheok YY, Saeidi H, Wong WF, Lee CYQ, Cheong HC, Yong YK, Larsson M, Shankar EM: T-Cell Exhaustion in Chronic Infections: Reversing the State of Exhaustion and Reinvigorating Optimal Protective Immune Responses. Front Immunol 2018, 9:2569.

[42] Alto NM, Orth K: Subversion of cell signaling by pathogens. Cold Spring Harb Perspect Biol 2012, 4:a006114.

[43] Gobert AP, Latour YL, Asim M, Finley JL, Verriere TG, Barry DP, Milne GL, Luis PB, Schneider C, Rivera ES, et al: Bacterial Pathogens Hijack the Innate Immune Response by Activation of the Reverse Transsulfuration Pathway. mBio 2019, 10.

[44] Pachathundikandi SK, Tegtmeyer N, Backert S: Signal transduction of Helicobacter pylori during interaction with host cell protein receptors of epithelial and immune cells. Gut Microbes 2013, 4:454-474. 
[45] Stutz MD, Clark MP, Doerflinger M, Pellegrini M: Mycobacterium tuberculosis:

Rewiring host cell signaling to promote infection. J Leukoc Biol 2018, 103:259-268.

[46] Krachler AM, Woolery AR, Orth K: Manipulation of kinase signaling by bacterial pathogens. J Cell Biol 2011, 195:1083-1092.

[47] Opitz B, van Laak V, Eitel J, Suttorp N: Innate immune recognition in infectious and noninfectious diseases of the lung. Am J Respir Crit Care Med 2010, 181:1294-1309.

[48] Green WD, Beck MA: Obesity altered T cell metabolism and the response to infection. Curr Opin Immunol 2017, 46:1-7.

[49] Lisowska B, Maśliński W, Małdyk P, Zabek J, Baranowska E: The role of cytokines in inflammatory response after total knee arthroplasty in patients with rheumatoid arthritis. Rheumatol Int 2008, 28:667-671.

[50] Roma P, Amandeep G, Pankaj B, Ishwarlal J: StatPearls. In; 2020

[51] Sloan M, Premkumar A, Sheth NP: Projected Volume of Primary Total Joint Arthroplasty in the U.S., 2014 to 2030. J Bone Joint Surg Am 2018, 100:1455-1460.

[52] Tande AJ, Patel R: Prosthetic joint infection. Clin Microbiol Rev 2014, 27:302-345.

[53] Tande AJ, Gomez-Urena EO, Berbari EF, Osmon DR: Management of Prosthetic Joint Infection. Infect Dis Clin North Am 2017, 31:237-252.

[54] Wildeman P, Tevell S, Eriksson C, Lagos AC, Söderquist B, Stenmark B: Genomic characterization and outcome of prosthetic joint infections caused by Staphylococcus aureus. Sci Rep 2020, 10:5938. 
[55] Grant SS, Hung DT: Persistent bacterial infections, antibiotic tolerance, and the oxidative stress response. Virulence 2013, 4:273-283.

[56] Shah K, Mohammed A, Patil S, McFadyen A, Meek RM: Circulating cytokines after hip and knee arthroplasty: a preliminary study. Clin Orthop Relat Res 2009, 467:946-951.

[57] Campoccia D, Mirzaei R, Montanaro L, Arciola CR: Hijacking of immune defences by biofilms: a multifront strategy. Biofouling 2019, 35:1055-1074.

[58] Gomes LSM: Early Diagnosis of Periprosthetic Joint Infection of the Hip-Current Status, Advances, and Perspectives. Rev Bras Ortop (Sao Paulo) 2019, 54:368-376.

[59] Parvizi J, Gehrke T, Chen AF: Proceedings of the International Consensus on Periprosthetic Joint Infection. Bone Joint J 2013, 95-B:1450-1452.

[60] Izakovicova P, Borens O, Trampuz A: Periprosthetic joint infection: current concepts and outlook. EFORT Open Rev 2019, 4:482-494.

[61] Cochran AR, Ong KL, Lau E, Mont MA, Malkani AL: Risk of Reinfection After Treatment of Infected Total Knee Arthroplasty. J Arthroplasty 2016, 31:156-161.

[62] Li C, Renz N, Trampuz A, Ojeda-Thies C: Twenty common errors in the diagnosis and treatment of periprosthetic joint infection. Int Orthop 2020, 44:3-14.

[63] Frangiamore SJ, Siqueira MB, Saleh A, Daly T, Higuera CA, Barsoum WK: Synovial Cytokines and the MSIS Criteria Are Not Useful for Determining Infection Resolution After Periprosthetic Joint Infection Explantation. Clin Orthop Relat Res 2016, 474:1630-1639.

[64] Vetter TR, Schober P, Mascha EJ: Diagnostic Testing and Decision-Making: Beauty Is Not Just in the Eye of the Beholder. Anesth Analg 2018, 127:1085-1091. 
[65] Schenk T, Irth H, Marko-Varga G, Edholm LE, Tjaden UR, van der Greef J: Potential of on-line micro-LC immunochemical detection in the bioanalysis of cytokines. J Pharm Biomed Anal 2001, 26:975-985.

[66] Clardy J: The chemistry of signal transduction. Proc Natl Acad Sci U S A 1995, 92:5661.

[67] Stenken JA, Poschenrieder AJ: Bioanalytical chemistry of cytokines--a review. Anal Chim Acta 2015, 853:95-115.

[68] Leng SX, McElhaney JE, Walston JD, Xie D, Fedarko NS, Kuchel GA: ELISA and multiplex technologies for cytokine measurement in inflammation and aging research. J Gerontol A Biol Sci Med Sci 2008, 63:879-884.

[69] Luminex xMAP Technology [https://www.bio-rad.com/featured/en/luminex-xmaptechnology.html\#: :text=Luminex\%20Overview,spectral\%20addresses\%20(color\%20codes).]

[70] Fu Q, Zhu J, Van Eyk JE: Comparison of multiplex immunoassay platforms. Clin Chem 2010, 56:314-318.

[71] Young SH, Antonini JM, Roberts JR, Erdely AD, Zeidler-Erdely PC: Performance evaluation of cytometric bead assays for the measurement of lung cytokines in two rodent models. J Immunol Methods 2008, 331:59-68.

[72] Vrana J: Utilizing early cellular changes to explore biological responses to individual chemical and mixtures exposures. ProQuest LLC; 2015.

[73] Athari SS: Targeting cell signaling in allergic asthma. Signal Transduct Target Ther 2019, 4:45. 
[74] Troy NM, Hollams EM, Holt PG, Bosco A: Differential gene network analysis for the identification of asthma-associated therapeutic targets in allergen-specific T-helper memory responses. BMC Med Genomics 2016, 9:9.

[75] Yang S, Liu G: Targeting the Ras/Raf/MEK/ERK pathway in hepatocellular carcinoma. Oncol Lett 2017, 13:1041-1047.

[76] Zhou Q, Lui VW, Yeo W: Targeting the PI3K/Akt/mTOR pathway in hepatocellular carcinoma. Future Oncol 2011, 7:1149-1167.

[77] Vilchez V, Turcios L, Marti F, Gedaly R: Targeting Wnt/ $\beta$-catenin pathway in hepatocellular carcinoma treatment. World J Gastroenterol 2016, 22:823-832.

[78] Dimri M, Satyanarayana A: Molecular Signaling Pathways and Therapeutic Targets in Hepatocellular Carcinoma. Cancers (Basel) 2020, 12.

[79] Han ZJ, Xue WW, Tao L, Zhu F: Identification of novel immune-relevant drug target genes for Alzheimer's Disease by combining ontology inference with network analysis. CNS Neurosci Ther 2018, 24:1253-1263.

[80] Barabási AL, Gulbahce N, Loscalzo J: Network medicine: a network-based approach to human disease. Nat Rev Genet 2011, 12:56-68.

[81] Yu D, Kim M, Xiao G, Hwang TH: Review of biological network data and its applications. Genomics Inform 2013, 11:200-210.

[82] Chen SJ, Liao DL, Chen CH, Wang TY, Chen KC: Construction and Analysis of ProteinProtein Interaction Network of Heroin Use Disorder. Sci Rep 2019, 9:4980. 
[83] Santiago JA, Bottero V, Potashkin JA: Transcriptomic and Network Analysis Highlight the Association of Diabetes at Different Stages of Alzheimer's Disease. Front Neurosci 2019, $13: 1273$

[84] Toubiana D, Puzis R, Wen L, Sikron N, Kurmanbayeva A, Soltabayeva A, Del Mar Rubio Wilhelmi M, Sade N, Fait A, Sagi M, et al: Combined network analysis and machine learning allows the prediction of metabolic pathways from tomato metabolomics data. Commun Biol 2019, 2:214.

[85] Crutchfield CA, Thomas SN, Sokoll LJ, Chan DW: Advances in mass spectrometrybased clinical biomarker discovery. Clin Proteomics 2016, 13:1.

[86] McDermott JE, Wang J, Mitchell H, Webb-Robertson BJ, Hafen R, Ramey J, Rodland KD: Challenges in Biomarker Discovery: Combining Expert Insights with Statistical Analysis of Complex Omics Data. Expert Opin Med Diagn 2013, 7:37-51.

[87] Hasan S, Bonde BK, Buchan NS, Hall MD: Network analysis has diverse roles in drug discovery. Drug Discov Today 2012, 17:869-874.

[88] Alyass A, Turcotte M, Meyre D: From big data analysis to personalized medicine for all: challenges and opportunities. BMC Med Genomics 2015, 8:33.

[89] Zhang P, Itan Y: Biological Network Approaches and Applications in Rare Disease Studies. Genes (Basel) 2019, 10.

[90] Ng A, Bursteinas B, Gao Q, Mollison E, Zvelebil M: Resources for integrative systems biology: from data through databases to networks and dynamic system models. Brief Bioinform 2006, 7:318-330. 
[91] Fabregat A, Jupe S, Matthews L, Sidiropoulos K, Gillespie M, Garapati P, Haw R, Jassal B, Korninger F, May B, et al: The Reactome Pathway Knowledgebase. Nucleic Acids Res 2018, 46:D649-D655.

[92] Kanehisa M, Furumichi M, Tanabe M, Sato Y, Morishima K: KEGG: new perspectives on genomes, pathways, diseases and drugs. Nucleic Acids Res 2017, 45:D353-D361.

[93] Pomaznoy M, Ha B, Peters B: GOnet: a tool for interactive Gene Ontology analysis. BMC Bioinformatics 2018, 19:470.

[94] Cirillo E, Parnell LD, Evelo CT: A Review of Pathway-Based Analysis Tools That Visualize Genetic Variants. Front Genet 2017, 8:174.

[95] Shannon P, Markiel A, Ozier O, Baliga NS, Wang JT, Ramage D, Amin N, Schwikowski B, Ideker T: Cytoscape: a software environment for integrated models of biomolecular interaction networks. Genome Res 2003, 13:2498-2504.

[96] Wang J, Sen S: MicroRNA functional network in pancreatic cancer: from biology to biomarkers of disease. J Biosci 2011, 36:481-491.

[97] Kaneko Y, Yatagai Y, Yamada H, Iijima H, Masuko H, Sakamoto T, Hizawa N: The search for common pathways underlying asthma and COPD. Int J Chron Obstruct Pulmon Dis 2013, 8:65-78.

[98] Si Y, Cui X, Crossman DK, Hao J, Kazamel M, Kwon Y, King PH: Muscle microRNA signatures as biomarkers of disease progression in amyotrophic lateral sclerosis. Neurobiol Dis 2018, 114:85-94. 
[99] Scardoni G, Laudanna C: Centralities Based Analysis of Complex Networks. In New Frontiers in Graph Theory. Edited by Zhang Y: Intech Open; 2012

[100] Han AA, Currie HN, Loos MS, Scardoni G, Miller JV, Prince N, Mouch JA, Boyd JW:

The impact of cytokine responses in the intra- and extracellular signaling network of a traumatic injury. Cytokine 2018, 106:136-147.

[101] Worley B, Powers R: Multivariate Analysis in Metabolomics. Curr Metabolomics 2013, 1:92-107.

[102] Reinke SN, Galindo-Prieto B, Skotare T, Broadhurst DI, Singhania A, Horowitz D, Djukanović R, Hinks TSC, Geladi P, Trygg J, Wheelock CE: OnPLS-Based Multi-Block Data Integration: A Multivariate Approach to Interrogating Biological Interactions in Asthma. Anal Chem 2018, 90:13400-13408.

[103] Groß A, Kracher B, Kraus JM, Kühlwein SD, Pfister AS, Wiese S, Luckert K, Pötz O, Joos T, Van Daele D, et al: Representing dynamic biological networks with multi-scale probabilistic models. Commun Biol 2019, 2:21.

[104] Williams JR, Yang R, Clifford JL, Watson D, Campbell R, Getnet D, Kumar R, Hammamieh R, Jett M: Functional Heatmap: an automated and interactive pattern recognition tool to integrate time with multi-omics assays. BMC Bioinformatics 2019, 20:81. 


\section{Chapter 2}

Localized Cytokine Responses to Total Knee Arthroplasty and Total Knee Revision Complications 


\section{Localized Cytokine Responses to Total Knee Arthroplasty and Total Knee Revision Complications $^{1}$}

The study of localized immune-related factors has proven beneficial for a variety of conditions, and one area of interest in the field of orthopaedics is the impact of implants and localized infections on immune response. Several cytokines have shown increased systemic concentrations in serum/plasma due to implants and infection, but tissue-level cytokines have not been investigated as thoroughly. This exploratory study investigated tissue-level cytokines in a cohort of patients $(\mathrm{N}=17)$ in response to total knee arthroplasty and total knee revision to better understand the immune response to implants and localized infection (e.g., prosthetic joint infection). The overall goal of this study was to provide insight into the localized cytokine response of tissues and identify tissue-level markers specific to inflammation caused by implants versus inflammation caused by infection. Tissues were collected across several anatomical locations and assayed with a panel of twenty human inflammatory cytokines to understand spatial differences in cytokine levels. In this study, six cytokines were elevated in implanted joints, as compared to native joints: IL-10, IL-12p70, IL-13, IL-17A, IL-4, and TNF- $\alpha$ ( $p<0.05)$. Seven cytokines showed infection-dependent increases in localized tissues: IL-1 $\alpha$, IL-1 $\beta$, IL-6, IL-8, MCP-1, MIP-1 $\alpha$, and MIP-1 $\beta(p<0.05)$. This study demonstrated that differences exist in tissue-level cytokines in response to presence of implant, and some cytokines were specifically elevated for infection; these responses may be informative of overall tissue health. These results highlight the utility of investigating localized cytokine concentrations to offer novel insights for total knee arthroplasty and total knee revision procedures, as well as their complications. Ultimately, this information

\footnotetext{
1 Parts of this chapter have been published previously from Prince N, Penatzer JA, Dietz, MJ, and Boyd, JW. Localized Cytokine Responses to Total Knee Arthroplasty and Total Knee Revision Complications. Journal of Translational Medicine. 18, 330 (2020).
} 
could provide additional, quantitative measurements of tissue to aid clinical decision making and patient treatment options.

\subsection{Introduction}

The inflammatory response to infection involves a series of biological events regulated by a number of immune mediators, and the actions of these immune factors are partially reliant on the cytokines and chemokines produced in response to pathogens, foreign bodies, and other stimuli [1-3]. These responses are of interest to the field of orthopaedics, especially with regard to the immune response to implants, infection, and chronic inflammation [4-6]. An elevated immune response has been observed following total knee arthroplasty (TKA) procedures, and increased levels of cytokines, particularly interleukin (IL)-1, IL-4, IL-6, IL-10, and tumor necrosis factor alpha (TNF- $\alpha$ ), have been observed on a systemic level (i.e., serum/plasma) as well as on a more localized level (i.e., synovial fluid) [7-9]. However, many aspects of this response are not well understood, so the inflammatory response in orthpaedic implants and implant infections remain uncharacterized. A majority of TKA procedures are successful, but implant-related and infectionrelated complications can negatively affect a patient's quality of life. Properly addressing these issues is of high priority to the field of orthopaedics, especially considering the increasing demand for joint replacement [10]. Many studies have noted the pain, inflammation, and dissatisfaction that can occur following these procedures, affecting approximately $20 \%$ of patients undergoing TKA $[11,12]$, but it is not entirely known what role cytokines play in this chronic inflammatory response.

Infections, such as prosthetic joint infection (PJI), are serious complications and the source of excessive joint inflammation, leading to higher rates of total joint failure [13]. PJI is a localized 
infection surrounding a prosthetic joint and can result following implantation, often necessitating surgical intervention [14]. PJI is a major concern following TKA/total knee revision (TKR) procedures and can be difficult to treat. The infections are often persistent and unable to be resolved using conventional methods, presenting a challenge for clinicians [15]. The systemic immune response to PJI has been studied extensively, but the localized tissue response is not as well understood. In order to better understand the immune response to implants and localized infection, this study investigated levels of twenty inflammatory cytokines in localized tissue surrounding the joint. While defining the localized response to implants and infection can be difficult [7-9], localized cytokine responses have been investigated for other pathological conditions. A few studies have characterized localized cytokine responses in trauma [16-18] and respiratory infection [19], and these studies demonstrated that the local cytokine environment differs when compared to systemically circulating levels. Currie et al. showed that differences in cytokine concentrations exist in skeletal muscle samples in a spatially-dependent manner using an animal model of traumatic injury [16]. Similarly, Hauser et al. observed differences in levels of cytokines at the site of injury compared to systemic levels in response to trauma in humans [18]. Other research groups have observed spatially-related differences of other immune-related factors for stroke [20], and in response to allergens [21] in animal models. These studies introduced the concept of using immune markers on a localized level to better understand these conditions.

TKA and TKR procedures trigger inflammatory cascades, initiating cytokine responses and elevating systemic cytokine concentrations; higher levels of cytokines have been observed following these surgeries. The elevation in cytokine levels has been attributed to the trauma of surgery as well as the introduction of implants into the body [22, 23]. However, this inflammation 
is sometimes prolonged, which can cause major complications for patients. The causes of chronic inflammation following these procedures are still unknown, and resolution of the inflammation is challenging [24]. Therefore, understanding the changes in inflammatory response specific to implant-related inflammation is beneficial to improving the outcome of these individuals.

Tissue-level response to PJI has not been characterized to understand the local immune modulation in these cases. Many studies have investigated systemically circulating levels of interleukins and other cytokines for their roles in infection, and several cytokines are used as diagnostics of PJI [25-27]. Several studies have specifically focused on the utility of measuring IL-6 and IL-8 levels in serum for diagnosing and monitoring PJI, both of which have increased specificity over conventional methods; this knowledge has greatly benefitted the clinical treatment options for PJI [28, 29]. However, PJI remains one of the most serious complications following revision knee arthroplasty. In fact, infection is one of the most common causes for revision, being implicated in 20.4\% of all revision TKA procedures between 2009 and 2013 [30]. While defining the systemic response to sepsis and infection has paved the way for improved diagnostics [31-33], less is known about the environment of localized infections and what role cytokines play in determining tissue health.

The present study focused on understanding differences in localized distributions of cytokines in TKA and TKR procedures, with and without presence of infection, using PJI as the model for localized infections. The ultimate goal of this study was to characterize the immune modulation on a tissue level that occurs in response to joint implantation and infection to better 
understand localized tissue health. The information gained could aid clinical management of these complications by narrowing down cytokines that are indicative of response to PJI. It represents the first known investigation of tissue-level cytokines in response to implant-related and infectionrelated complications, to our knowledge.

\subsection{Materials and methods}

\subsubsection{Patient cohort}

Following Institutional Review Board (IRB) approval (IRB Protocol \#1709745853) and patient consent, six patients undergoing primary total knee arthroplasty (TKA) and eleven patients undergoing total knee revision (TKR) procedures participated in the study (8 males, 9 females; aged 45-82 years; body max index [BMI] 24.6-43.7). Subjects were recruited over a 12-month period. All six primary TKA patients were undergoing elective surgery for total replacement of the knee joint with a diagnosis of osteoarthritis. At the time of this study, this was the first arthroplasty procedure on either knee joint. In the TKR group, patients were further characterized into aseptic and septic revision procedures. Patients with aseptic revisions $(\mathrm{N}=5)$ were undergoing revisions due to failures of the prosthetic joint but did not show presence of infection. For ease of the reader, samples from these patients will be referred to as aseptic TKR tissues. Patients with septic revisions $(\mathrm{N}=6)$ met clinical criteria for a PJI diagnosis as defined by the Musculoskeletal Infection Society (MSIS) criteria [13]. Samples from these patients will be referred to as septic TKR tissues. All six patients diagnosed with PJI were tissue culture positive: four tested culture positive for Staphylococcus epidermidis, one for Methicillin-sensitive Staphylococcus aureus (MSSA), and one for Enterobacter cloacae. More patient information can be found in Table 2.1 below. Systemic C-reactive protein (CRP) levels in serum are additionally listed as reference. 
Table 2. 1. Patient Information. Six primary TKA and eleven revision TKR patients were enrolled in the study, creating a heterogenous cohort of males and females varying in age (45-82 years) and comorbidities. Primary TKA patients have ID format P\#; revision TKR patients have ID format F\#. This table lists general patient information including the pathogen for which each septic patient tested culture-positive following testing on the day of surgery. Serum CRP values were obtained pre-operatively in the revision setting. Cultures were obtained from intraoperative tissue samples.

\begin{tabular}{|c|c|c|c|c|c|c|}
\hline ID & Sex & TKA/TKR & BMI $\left(\mathrm{kg} / \mathrm{m}^{2}\right)$ & Diabetic $(\mathbf{Y} / \mathbf{N})$ & CRP (mg/L) & Culture \\
\hline $\mathrm{P} 1$ & $\mathrm{~F}$ & TKA & 33.8 & $\mathrm{~N}$ & N/A & Negative \\
\hline $\mathrm{P} 2$ & $\mathrm{~F}$ & TKA & 39.8 & $\mathrm{~N}$ & N/A & Negative \\
\hline P3 & $\mathrm{F}$ & TKA & 39.8 & $\mathrm{~N}$ & N/A & Negative \\
\hline $\mathrm{P} 4$ & $\mathrm{M}$ & TKA & 29.7 & $\mathrm{Y}$ & N/A & Negative \\
\hline P5 & $\mathrm{M}$ & TKA & 24.6 & $\mathrm{~N}$ & N/A & Negative \\
\hline P6 & $\mathrm{M}$ & TKA & 27.2 & $\mathrm{~N}$ & N/A & Negative \\
\hline F1 & $\mathrm{F}$ & TKR- Aseptic & 28.2 & $\mathrm{~N}$ & 4.3 & Negative \\
\hline $\mathrm{F} 2$ & $\mathrm{~F}$ & TKR- Aseptic & 29.8 & $\mathrm{~N}$ & 0.2 & Negative \\
\hline F3 & $\mathrm{F}$ & TKR-Aseptic & 33.9 & $\mathrm{~N}$ & $<1$ & Negative \\
\hline F4 & $\mathrm{M}$ & TKR-Aseptic & 40.4 & $\mathrm{Y}$ & 3.6 & Negative \\
\hline F5 & $\mathrm{M}$ & TKR- Aseptic & 26.2 & $\mathrm{~N}$ & 2.1 & Negative \\
\hline F6 & $\mathrm{F}$ & TKR-Septic & 43.7 & $\mathrm{~N}$ & 28.8 & S. epidermidis \\
\hline F7 & $\mathrm{F}$ & TKR-Septic & 30.8 & $\mathrm{Y}$ & 161.4 & S. epidermidis \\
\hline F8 & $\mathrm{F}$ & TKR-Septic & 41.9 & $\mathrm{~N}$ & 21.7 & E. cloaecae \\
\hline F9 & M & TKR-Septic & 36.2 & $\mathrm{~N}$ & 33.5 & $M S S A$ \\
\hline F10 & $\mathrm{M}$ & TKR-Septic & 33.8 & $\mathrm{Y}$ & 3.8 & S. epidermidis \\
\hline F11 & $\mathrm{M}$ & TKR-Septic & 31.9 & $\mathrm{~N}$ & 111.9 & S. epidermidis \\
\hline
\end{tabular}




\subsubsection{Collection of tissue samples}

All TKA and TKR procedures were performed by a single surgeon with standard debridement and washing protocols. Tissues were collected at a total of four distinct anatomical locations, broadly characterized into two tissue layers: four adjacent tissue layer (ATL) samples and three radial tissue layer (RTL) samples. The ATL samples came from the initial debridement. Tissues from the ATL layer were closer to the knee joint (or prosthetic implant). Conversely, RTL samples were taken from a tissue layer further removed from the joint (or prosthetic implant) after the surgeon completed debridement. The difference in depth of the RTL tissues and ATL tissues was approximately 5-10 $\mathrm{mm}$ and was dependent on the individual patient. Measurements were made from point of origin to standardize tissue samples taken between patients. Tissues were taken at four anatomical locations illustrated in Figure 2.1. Briefly, the solid line circle represents location 1) medial femoral condyle (F); the dashed line circle represents location 2) medial tibial plateau (T); the solid line square represents location 3) lateral gutter (LG); and the dashed line square represents location 4) posterior capsule (PC). Anatomical locations 1-4 were collected for the ATL layer, and locations 1-3 were collected for the RTL layer. Location 4, PC, could not be taken in the RTL layer due to proximity to neurovascular structures. Therefore, a total of seven tissue samples were taken for each patient. 


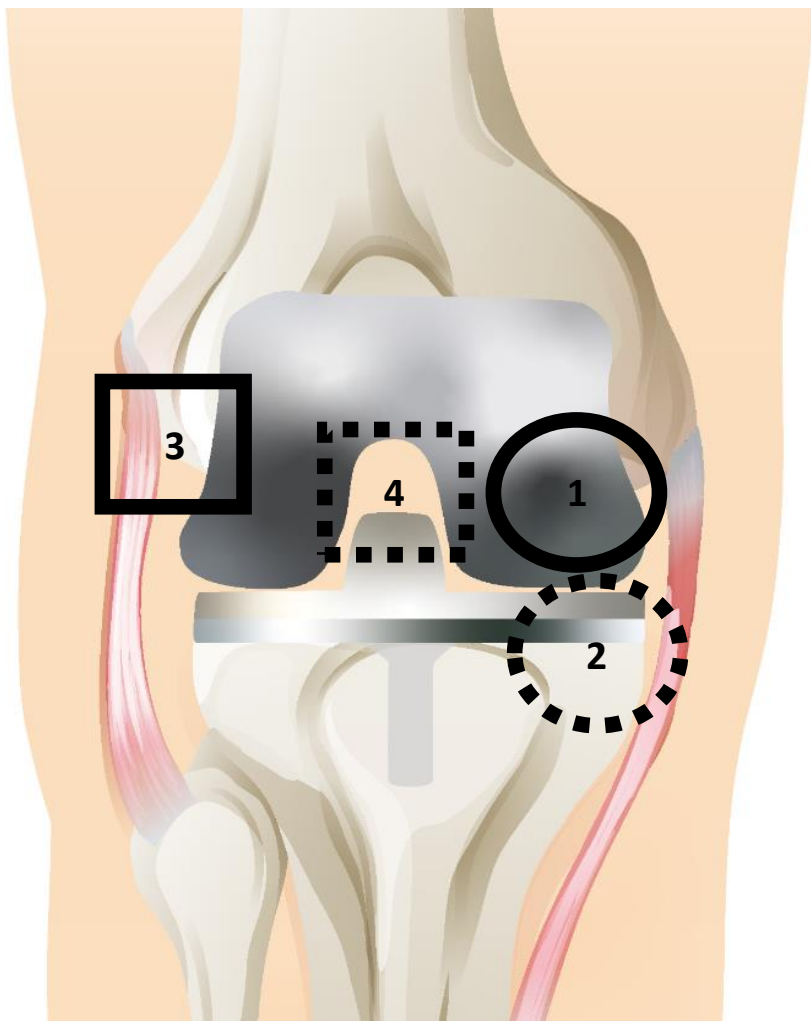

Figure 2. 1. Map of approximate tissue collection locations, shown with prosthetic implant illustrated. Seven tissue samples were taken for each patient; 1) the solid circle represents the medial femoral condyle (denoted as F); 2) the dashed circle represents the medial tibial plateau (denoted as T); 3) the solid square represents the lateral gutter (denoted as LG); 4) the dashed square represents the posterior capsule (denoted as PC). Locations 1-4 were taken for the ATL layer, and locations 1-3 were taken for the RTL layer; separation between ATL (closer to joint) and RTL (further from joint) was approximately $5-10 \mathrm{~mm}$, depending on individual patient.

\subsubsection{Sample preparation}

Tissues were collected during TKA and TKR procedures in the operating room and immediately stored on dry ice. Once all tissues had been collected for an individual patient, they were washed with $1 \mathrm{X}$ cold phosphate-buffered saline (PBS) to remove blood and debris. Tissues were grossly dissected using a scalpel to remove scar tissue or cement, then stored at $-80{ }^{\circ} \mathrm{C}$. When samples had been collected for all patients, tissues were thawed on ice and cut into sections approximately $30 \mathrm{mg}$ in size; tissues were homogenized by sonication in $500 \mu \mathrm{L}$ cell lysis solution 
(Bio-Rad, Hercules, CA) containing $20 \mathrm{mM}$ phenylmethylsulfonyl fluoride (Sigma-Aldrich, St. Louis, MO). Protein extraction was performed using methods adapted from Hulse et al. [34]. Thawed samples were vortexed for 1-3 s and centrifuged at 5,000 $\mathrm{xg}$ for 5 minutes at $4{ }^{\circ} \mathrm{C}$. The supernatant was collected and tested for total protein content using a Pierce BCA Protein Assay Kit (Thermo Scientific, Waltham, MA), according to manufacturer's instructions. Absorbance values for total protein content were determined on an Infinite M1000 multimode plate reader (Tecan, Raleigh, NC).

\subsubsection{Cytokine measurement}

To standardize samples for total protein content, tissue homogenates were individually diluted to a total protein concentration of $900 \mu \mathrm{g} / \mathrm{mL}$ with cell lysis buffer (Bio-Rad). Cytokine quantification was performed using a magnetic bead-based multiplex Inflammation Human ProcartaPlex panel assay (Invitrogen, Carlsbad, CA) and measured using a Bio-Plex 200 suspension array system and Pro II Wash Station (Bio-Rad), according to the manufacturer's instructions. Cytokine concentrations were averaged to represent values for the ATL and RTL. Four tissues were averaged to calculate ATL average (LG, F, T, PC), and three tissues were averaged to calculate RTL average (LG, F, T). A table of cytokine values at ATL and RTL and graphs of cytokine concentrations at individual tissue locations are available in Appendix A.

\subsubsection{Statistical analysis}

Data were analyzed using Prism 5 (GraphPad, San Diego, CA) and SAS JMP (Cary, NC). Standard curves were generated for each protein using either a four- (4PL) or five-parameter logistic (5PL) regression model, depending on the individual protein. Cytokine concentrations 
were determined using standard curve interpolation, then corrected by dilution factor to compare tissue homogenates. Cytokine concentrations are expressed as picograms of cytokine per milliliter of tissue homogenate $(\mathrm{pg} / \mathrm{mL})$. Samples with fluorescence intensity values below the lower limit of quantitation (LLOQ) or above the upper limit of quantitation (ULOQ) were omitted from statistical comparisons. Outliers were identified using the $1.5 \mathrm{X}$ interquartile range (IQR) rule and omitted from analysis. Two-way analysis of variance (ANOVA) with Bonferroni's post-test was used to determine significant differences between primary TKA, aseptic TKR, and septic TKR tissue samples at each tissue location. Each tissue homogenate was tested in duplicate for cytokine concentration. Data are expressed as the mean \pm standard error of the mean (SEM).

Quadratic discriminant analysis was conducted to evaluate the combined capacity of cytokine response to predict the state of tissue. Using SAS JMP, all measured responses were cast as covariates, and the "group" was assigned as a classification category (primary TKA, aseptic TKR, septic TKR). The Shrink Covariances option was applied to account for the different covariances within the categories. Quadratic discriminant analysis is a predictive modeling tool, and when there are a large number of variables compared to observations, as is the case in this study, Shrink Covariances is frequently employed to improve the stability and reduce prediction variance [35]. This analysis included 13 covariates; only those cytokines that produced statistically significant two-way ANOVA comparisons for either infection-specific or implant-specific comparisons were included: IL-1 $\alpha$, IL-1 $\beta$, IL-6, IL-8, monocyte chemoattractant protein (MCP)1, macrophage inflammatory protein (MIP)-1 $\alpha$, MIP-1 $\beta$, IL-10, IL-12p70, IL-13, IL-17A, and TNF- $\alpha$. Biplot rays are plotted to indicate how each covariate influences the canonical space, with 
the direction and magnitude signifying the degree of association with the respective group (primary TKA, aseptic TKR, septic TKR).

Due to the limited sample size, this study was not able to control for age, sex, BMI, or other comorbidities. Pearson correlations were run between cytokine concentrations and age, sex, and BMI for each patient to analyze the contribution of these variables. Bonferroni's correction was applied to correct for multiple inferences, as previously described by Bland et al. [36].

\subsection{Results}

Changes in cytokine concentrations were observed for comparisons of primary TKA vs. aseptic TKR vs. septic TKR tissues. Overall, cytokine concentrations were generally elevated in TKR (both septic and aseptic) compared to TKA, and septic TKR exhibited higher cytokine levels than aseptic TKR for several cytokines. Seven cytokines (IL-1 $\alpha$, IL-1 $\beta$, IL-6, IL-8, MCP-1, MIP$1 \alpha$, and MIP-1 $\beta$ ) showed increased concentrations in septic TKR tissues compared to both aseptic TKR tissues and primary TKA tissues (p<0.05). Six cytokines (IL-10, IL-12p70, IL-13, IL-17A, IL-4, and TNF- $\alpha$ ) showed differences in concentration between primary TKA and TKR (both aseptic and septic) $(\mathrm{p}<0.05)$, but these six cytokines were not significantly different between aseptic TKR and septic TKR. These comparisons are described in detail over the following sections. Additional human inflammatory cytokines were tested, but they did not produce statistically significant comparisons at $\mathrm{p}<0.05$ in this study: E-Selectin, granulocyte-macrophage colony-stimulating factor (GM-CSF), interferon-alpha (IFN- $\alpha$ ), interferon-gamma (IFN- $\gamma$ ), and interferon gamma-induced protein 10 (IP-10). 


\subsubsection{Seven cytokines exhibited infection-specific elevations in concentration}

Seven cytokines showed an increase in concentration that was dependent on the presence of localized infection: IL-1 $\alpha$, IL-1 $\beta$, IL-6, IL-8, MCP-1, MIP-1 $\alpha$, and MIP-1 $\beta(p<0.05)$. For these cytokines, primary TKA averages were lowest, with an increase in aseptic TKR and further increase in septic TKR. For IL-1 $\alpha$, the average concentration of primary TKA tissues was $1.1 \mathrm{pg} / \mathrm{mL}$, and rose to $11.8 \mathrm{pg} / \mathrm{mL}$ in aseptic TKR; the concentration was elevated to $30.3 \mathrm{pg} / \mathrm{mL}$ in septic TKR. Further, in the septic TKA group, there was a statistically significant difference between ATL and RTL averages ( $\mathrm{p}<0.05$ ). IL-1 $\beta$ showed a similar trend, with a mean of $1.7 \mathrm{pg} / \mathrm{mL}$ in primary TKA tissues, which rose to $5.4 \mathrm{pg} / \mathrm{mL}$ in aseptic TKR, and further elevated to $39.1 \mathrm{pg} / \mathrm{mL}$ in septic TKR. IL-1 $\beta$ also reflected the spatial disparity in concentration between ATL and RTL in the septic TKR group (Fig. 2.2). IL-6 followed, with an average of $8.5 \mathrm{pg} / \mathrm{mL}$ in primary TKA, rising to $24.2 \mathrm{pg} / \mathrm{mL}$ in aseptic TKR, and finally $610.7 \mathrm{pg} / \mathrm{mL}$ in septic TKR. IL-8 levels were $7.6 \mathrm{pg} / \mathrm{mL}$ in primary TKA, which increased to $91.1 \mathrm{pg} / \mathrm{mL}$ in aseptic TKR, and rose to $553.9 \mathrm{pg} / \mathrm{mL}$ in septic TKR. For MCP-1, the average of primary TKA tissues was $113.0 \mathrm{pg} / \mathrm{mL}$, which increased to $258.8 \mathrm{pg} / \mathrm{mL}$ for aseptic TKR, and further increased to $565.1 \mathrm{pg} / \mathrm{mL}$ for septic TKR. MIP- $1 \alpha$ followed the same trend, with an average of $7.8 \mathrm{pg} / \mathrm{mL}$ for primary TKA, which rose to $27.8 \mathrm{pg} / \mathrm{mL}$ in aseptic TKR, and was elevated to $81.6 \mathrm{pg} / \mathrm{mL}$ in septic TKR. ATL locations showed the most significant increases in MIP- $1 \alpha$ between groups (Fig. $2.2, \mathrm{p}<0.05$ ). For MIP-1 $\beta$,

primary TKA tissues showed an average of $21.3 \mathrm{pg} / \mathrm{mL}$ and were increased to $46.0 \mathrm{pg} / \mathrm{mL}$ for aseptic TKR and further increased to $123.4 \mathrm{pg} / \mathrm{mL}$ in septic TKR. As shown in Figure 2.2, cytokine concentrations in the ATL layer locations were generally higher than the RTL layer locations for all of these cytokines except MCP-1. 

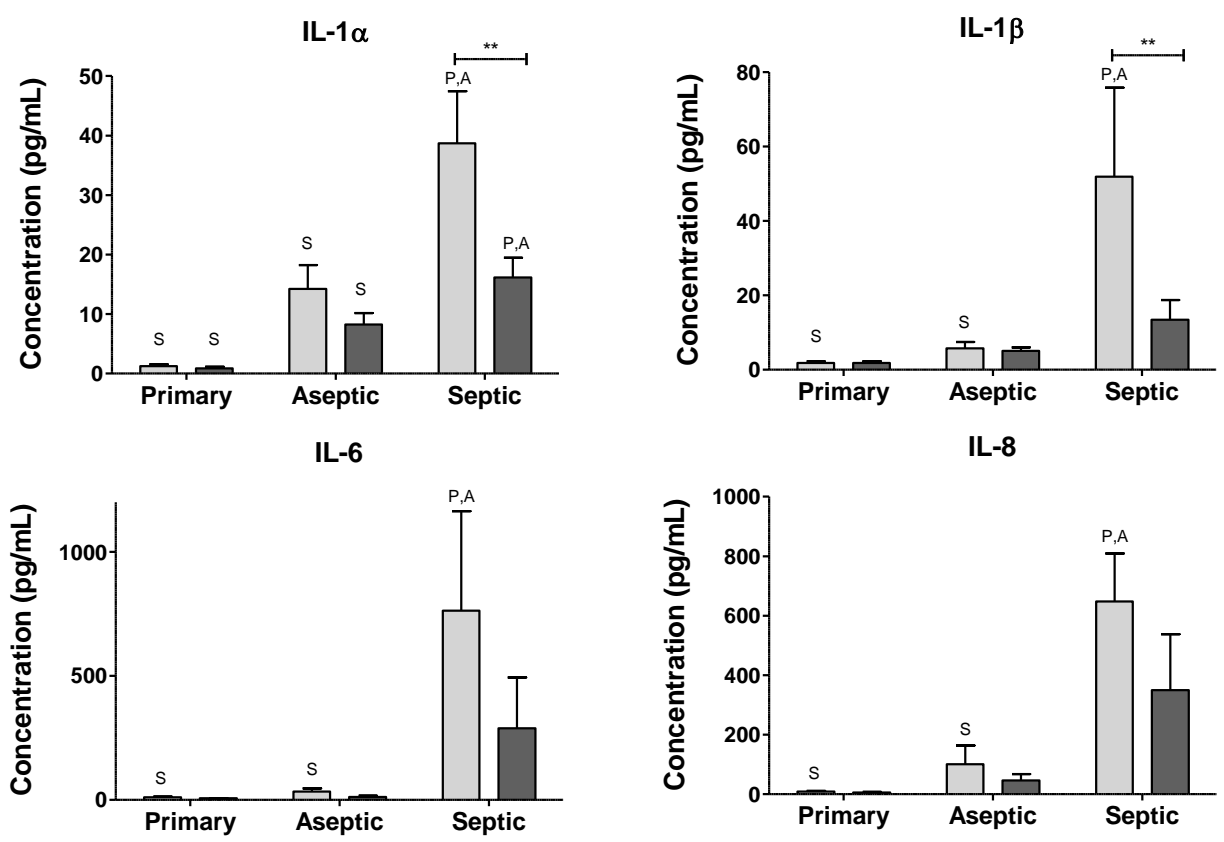

MIP-1 $\alpha$

MIP-1 $\beta$
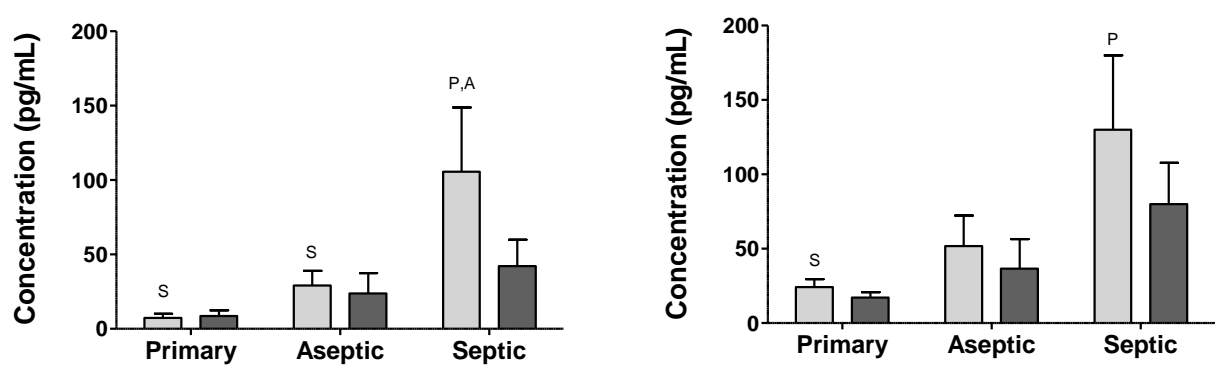

MCP-1

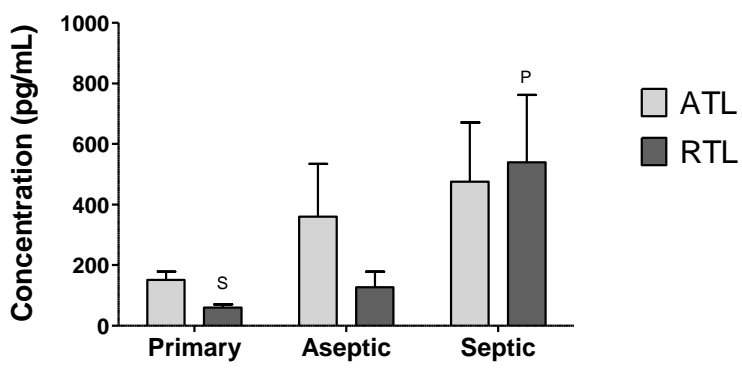

Figure 2. 2. Seven cytokines showed infection-dependent elevation in localized tissues. Average cytokine concentration for ATL and RTL are shown for all groups. Two-way ANOVAs with Bonferroni's post-test were conducted to test for significant differences between groups at each tissue depth $(\mathrm{p}<0.05)$. Significant differences between groups at a particular location are marked as: $\mathrm{P}$ denotes significant difference from primary TKA $(\mathrm{N}=6)$; A denotes significant difference from aseptic TKR $(\mathrm{N}=5)$; $\mathrm{S}$ denotes significant difference from septic TKR $(\mathrm{N}=6)$; all symbols denote significance at the $\mathrm{p}<0.05$ level. 


\subsubsection{Six cytokines exhibited implant-related elevations in concentration (Primary TKA vs. Aseptic/Septic TKR)}

Six cytokines, IL-10, IL-12p70, IL-13, IL-17A, IL-4, and TNF- $\alpha$, exhibited higher levels in TKR tissues as compared to primary TKA tissues ( Fig. 2.3 p <0.05). In other words, there were significant differences $(\mathrm{p}<0.05)$ between primary TKA and aseptic/septic TKR, but there were no significant elevations in concentration from aseptic TKR to septic TKR. For IL-10, the average value in primary TKA was $0.9 \mathrm{pg} / \mathrm{mL}, 8.4 \mathrm{pg} / \mathrm{mL}$ in aseptic TKR, and $6.6 \mathrm{pg} / \mathrm{mL}$ in septic TKR. With the same general trend, IL-12p70 had an average of $5.7 \mathrm{pg} / \mathrm{mL}$ in primary TKA, $30.7 \mathrm{pg} / \mathrm{mL}$ in aseptic TKR, and $20.7 \mathrm{pg} / \mathrm{mL}$ in septic TKR. For IL-13, the average in primary TKA was $1.8 \mathrm{pg} / \mathrm{mL}, 9.6 \mathrm{pg} / \mathrm{mL}$ in aseptic TKR, and $9.9 \mathrm{pg} / \mathrm{mL}$ in septic TKR. Following this trend, IL-17A average concentrations were $5.3 \mathrm{pg} / \mathrm{mL}$ in primary TKA, $16.3 \mathrm{pg} / \mathrm{mL}$ in aseptic TKR, and $18.9 \mathrm{pg} / \mathrm{mL}$ in septic TKR. For IL-4, average concentration in primary TKA was $6.9 \mathrm{pg} / \mathrm{mL}$, which rose to $19.6 \mathrm{pg} / \mathrm{mL}$ in aseptic TKR, and further to $24.8 \mathrm{pg} / \mathrm{mL}$ in septic TKR. Finally, TNF- $\alpha$ followed the same trend, with an average concentration of $16.9 \mathrm{pg} / \mathrm{mL}$ in primary TKA, $71.1 \mathrm{pg} / \mathrm{mL}$ in aseptic TKR, and $86.8 \mathrm{pg} / \mathrm{mL}$ in septic TKR. None of these six cytokines showed significant spatial disparities between the ATL and RTL layers at $\mathrm{p}<0.05$. 
IL-10

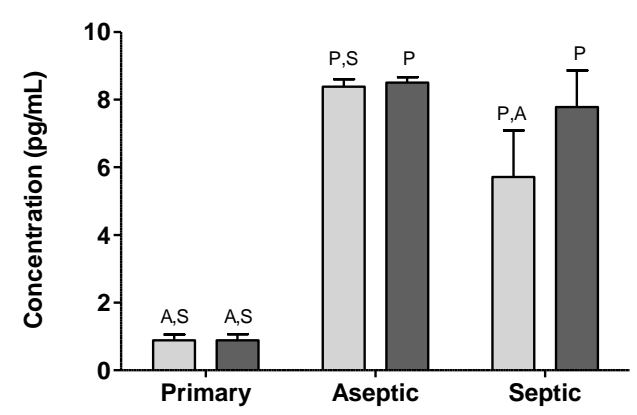

IL-17A

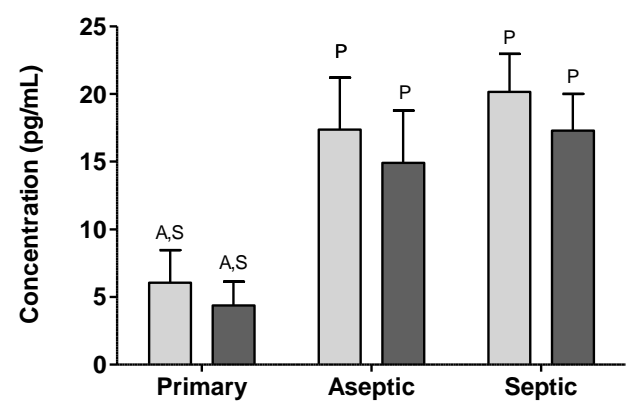

TNF- $\alpha$

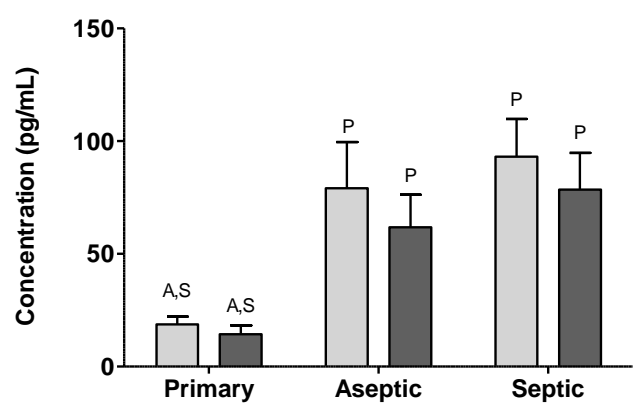

IL-13

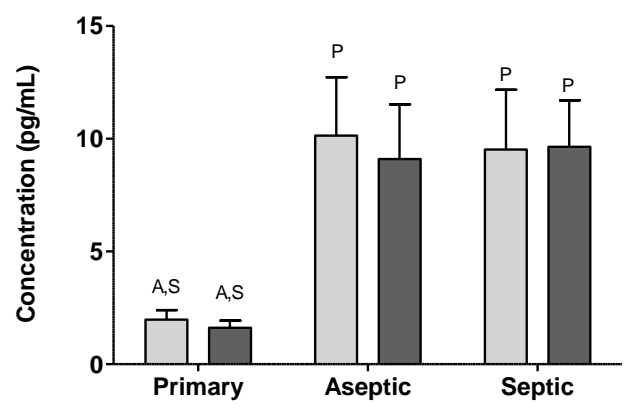

IL-4

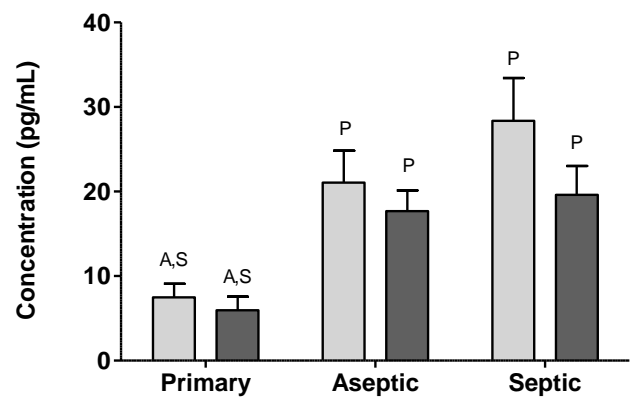

IL-12p70

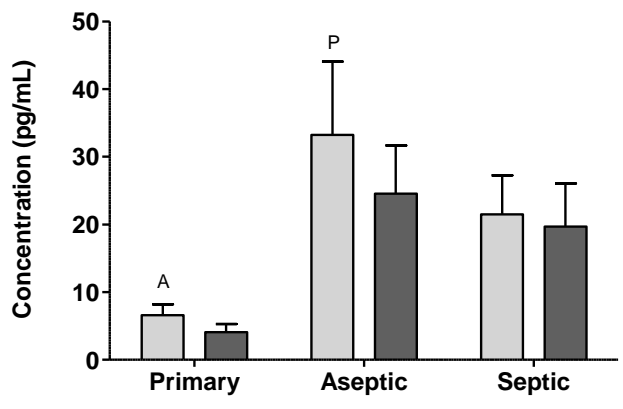

$\square$ RTL

Figure 2. 3. Six cytokines showed implant-related elevation in localized tissues that was not infection-dependent. Average cytokine concentrations for ATL and RTL are shown for all groups. Two-way ANOVAs with Bonferroni's post-test were conducted to test for significant differences between groups at each tissue depth $(\mathrm{p}<0.05)$. Significant differences between groups at a particular location are marked as: $\mathrm{P}$ denotes significant difference from primary TKA $(\mathrm{N}=6)$; A denotes significant difference from aseptic TKR $(\mathrm{N}=5)$; $\mathrm{S}$ denotes significant difference from septic TKR $(\mathrm{N}=6)$; all symbols denote significance at the $\mathrm{p}<0.05$ level. 


\subsubsection{Quadratic discriminant analysis (QDA) cytokine profiles for TKA vs. TKR}

The two-way ANOVA comparisons of cytokines between different groups revealed seven cytokines that showed infection-specific elevation (beyond inflammation caused by implants), and six cytokines that showed increases due to implants, but not infection (Figures 2.2 and 2.3). To further probe the structure of these cytokine profiles between groups, quadratic discriminant analysis was conducted. These thirteen cytokines were included as covariates. The analysis classified the combined observed responses into pre-determined groups of primary TKA, aseptic TKR, and septic TKR. The group was predicted based on the covariate responses associated with each group, respectively. For each group, all seven locations were included for all individuals in that group, which means there were 42 counts for primary TKA ( 7 tissue locations, 6 patients), 35 values for aseptic TKR ( 7 tissue locations, 5 patients), and 42 counts for septic TKR (7 tissue locations, 6 patients). In total, of 119 counts, only 8 were misclassified, indicating a good prediction ability of the model. All 8 misclassifications were errors of a prediction of aseptic TKR group, when the values were originally from the septic TKR group. In other words, these individuals were falsely classified as aseptic based on cytokine profiles while they were actually septic. Further, there is overlap between the $95 \%$ confidence intervals for cytokine profiles of aseptic TKR and septic TKR patients (Figure 2.4), which may be responsible for the misclassification. 


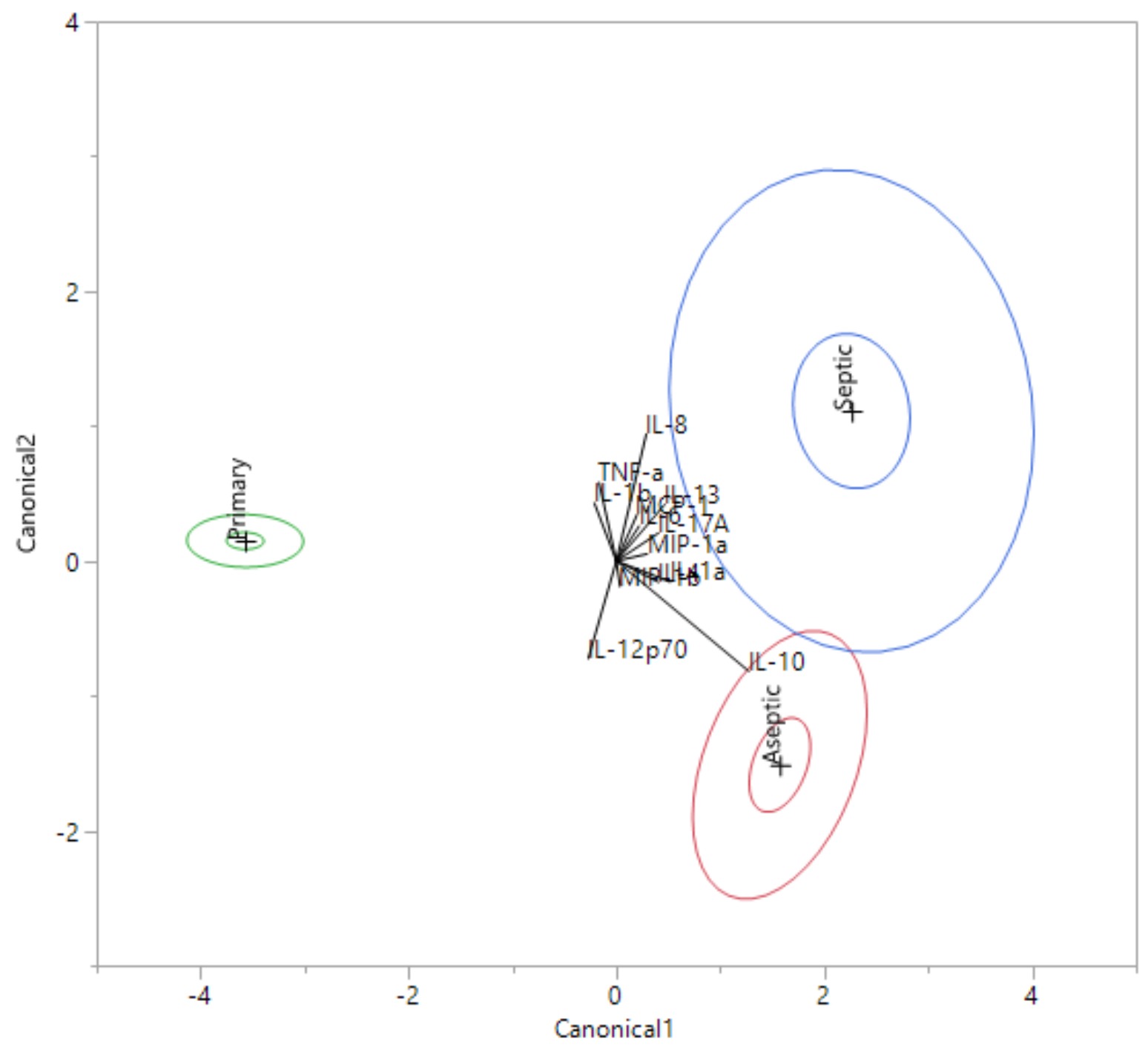

Figure 2. 4. Quadratic discriminant analysis (QDA) revealed distinct groupings for primary TKA vs. TKR (aseptic or septic). Cytokines with significant infection-dependent or implant-related elevations via two-way ANOVA were analyzed via quadratic discriminant analysis. Canonical scores for each cytokine (covariate) were calculated, and the 95\% confidence interval is shown for primary TKA (green), aseptic TKR (red), and septic TKR (blue). The + symbol represents the mean of each group. Biplot rays describe the degree of association of a certain cytokine with canonical variables. 


\subsubsection{Effects of age, sex, and BMI on cytokine concentrations}

The research presented here did not control for age, sex, or BMI due to the limited sample size of this exploratory study. To better understand the connections between cytokines of interest (IL-1 $\alpha$, IL-1 $\beta$, IL-6, IL-8, MCP-1, MIP-1 $\alpha$, MIP-1 $\beta$, IFN- $\gamma$, IL-10, IL-13, IL-17A, IL-4, and TNF-

$\alpha)$ and these factors, Pearson correlations were run and analyzed for statistical significance. When the Bonferroni's correction was applied, as described in [36], none of the correlations between cytokine levels and age, sex, or BMI were significant ( $p>0.05)$, but the correlations are displayed in Table 2.2 for transparency. Although there is an established connection in the literature between inflammatory cytokine levels and age, sex, and BMI, the lack of significant Pearson correlation pvalues indicates these were not confounding variables for this study [37-39]. 
Table 2. 2. Pairwise Pearson Correlation Values Between Cytokine Concentrations and Age, Sex, and $B M I$. The pairwise correlation values are listed for each of the three groups: primary TKA, aseptic TKR, and septic TKR. Pearson correlation values are rounded to two decimal places. No correlations were found to be significant at the $\mathrm{p}<0.05$ level after Bonferroni's correction.

\begin{tabular}{|c|c|c|c|c|c|c|c|c|c|}
\hline & \multicolumn{3}{|c|}{ Primary TKA } & \multicolumn{3}{c|}{ Aseptic TKR } & \multicolumn{3}{c|}{ Septic TKR } \\
\hline Cytokine & Age & Sex & BMI & Age & Sex & BMI & Age & Sex & BMI \\
\hline IL-1 $\alpha$ & 0.00 & 0.47 & 0.28 & 0.20 & 0.22 & -0.54 & -0.15 & 0.25 & -0.05 \\
\hline IL-1 $\beta$ & -0.19 & -0.06 & -0.04 & -0.30 & -0.30 & 0.08 & 0.03 & 0.26 & 0.05 \\
\hline IL-6 & 0.00 & -0.09 & -0.12 & 0.03 & -0.19 & -0.21 & 0.00 & 0.22 & -0.02 \\
\hline IL-8 & 0.03 & 0.59 & 0.25 & 0.06 & -0.07 & -0.32 & -0.13 & 0.14 & -0.14 \\
\hline MCP-1 & 0.04 & 0.13 & 0.14 & -0.18 & -0.44 & 0.45 & -0.07 & 0.20 & -0.15 \\
\hline MIP-1 $\alpha$ & -0.10 & 0.13 & -0.04 & -0.05 & -0.30 & 0.09 & 0.33 & 0.08 & -0.13 \\
\hline MIP-1 $\beta$ & -0.04 & 0.19 & 0.03 & 0.23 & -0.06 & -0.38 & 0.21 & 0.13 & -0.16 \\
\hline IL-10 & -0.26 & 0.29 & 0.17 & -0.31 & 0.00 & -0.20 & -0.11 & -0.25 & 0.30 \\
\hline IL-12p70 & -0.06 & 0.02 & 0.03 & -0.03 & 0.49 & -0.32 & -0.30 & -0.18 & 0.33 \\
\hline IL-13 & -0.19 & 0.49 & 0.28 & -0.06 & 0.41 & -0.06 & -0.22 & -0.23 & 0.22 \\
\hline IL-17A & 0.00 & -0.08 & -0.31 & 0.31 & 0.85 & -0.45 & -0.34 & 0.06 & 0.32 \\
\hline IL-4 & -0.32 & 0.20 & 0.29 & 0.34 & 0.35 & -0.55 & 0.21 & 0.02 & -0.11 \\
\hline TNF- $\alpha$ & -0.22 & 0.34 & 0.25 & 0.45 & 0.72 & -0.38 & -0.45 & -0.12 & 0.25 \\
\hline
\end{tabular}




\subsection{Discussion}

Understanding inflammation in response to implants and infection following TKA and TKR procedures is a high priority for clinicians, as excessive inflammation can cause serious problems for patients. However, not much is known about the local immune response in these complex environments. While a variety of cytokines have been researched from a systemic view $[40,41]$, their clinical use is still debated [31-33, 42], and the cytokine responses have not been as well characterized on a localized tissue level. The tissue-level cytokine response may add further understanding of the localized environment and give insight into tissue health to aid clinicians in the management of these post-surgical complications. Tissue-level cytokines have been measured with respect to spatial gradients in traumatic injury [16-18], respiratory infection [19], stroke [20], and allergic response [21], and these studies provided useful information regarding the respective immune responses. These have established a basis for this study to investigate the use of cytokines to enlighten tissue viability following localized implant-related and infection-specific inflammation.

This study focused on defining the tissue-level cytokine response to implants and infection across several anatomical locations. Many human inflammatory cytokines have been implicated in the systemic response to implants (i.e., in serum/plasma) [43-45] and now aid in diagnosis of infection $[46,47]$. However, this investigation is the first, to our knowledge, to assess multiple tissue locations surrounding the joint to address implant-related vs. infection-specific responses. Seven cytokines were identified as infection-specific, showing elevated concentrations in the septic TKR cohort compared to both the aseptic TKR and primary TKA cohorts: IL-1 $\alpha$, IL-1 $\beta$, IL6, IL-8, MCP-1, MIP-1 $\alpha$, and MIP-1 $\beta$ ( $\mathrm{p}<0.05)$. Several of these cytokines have demonstrated 
utility in previous studies for diagnosis of PJI (i.e., IL-1 $\alpha$, IL-1 $\beta$, IL-6, IL-8), but this is the first instance of their investigation for tissue health and debridement [30-33]. Generally, these seven cytokines were elevated in the ATL depth compared to RTL, which brings to light the importance of proximity to joint in dictating cytokine response. Pro-inflammatory cytokines like IL-1 $\alpha$, IL-1 $\beta$, IL-6, and IL-8 have been noted for their roles in early infection response, producing a warning signal of pathogen invasion, and this response was present in septic TKR tissues [48, 49]. These early cytokine indicators recruit factors like MCP-1, MIP-1a, and MIP-1b that propagate the response to pathogens through Th1 and Th2 immune signaling cascades [50, 51]. IL-1 $\alpha$ and IL-1 $\beta$ in particular showed a statistically significant elevation in the ATL of the septic TKR group compared to the RTL of the septic TKR group, which suggested that these cytokines may be capable of distinguishing healthy and unhealthy tissue in PJI.

Six cytokines were showed implant-related increases in concentration, with elevations in aseptic and septic TKR vs. primary TKA: IL-10, IL-12p70, IL-13, IL-17A, IL-4, and TNF- $\alpha$ $(p<0.05)$. The elevated concentrations of these cytokines highlighted the degree of inflammation in implanted joints without the presence of infection, which is likely due to the presence of a foreign body. The implant-related inflammation reflected less of the macrophage activation present in the septic TKR group but exhibited elevation in anti-inflammatory cytokines like IL-10, IL-4, and IL-13 frequently associated with bone healing [7]. IL-17A and IL-12p70 have both proand anti-inflammatory roles, but the specific contributions to foreign body response are not well understood. Increased levels of these cytokines, as well as TNF- $\alpha$, implies there may be dysregulation of inflammatory response due to implant. These cytokines were not significantly different in the septic TKR group compared to aseptic TKR at the $p<0.05$ level, so they may be 
considered as indicators of aseptic or chronic inflammation that could be addressed with future research associated with TKA. The QDA analysis illustrated that cytokine profiles are distinct between all three cohorts, but there is significant overlap in the $95 \%$ confidence intervals of aseptic TKR and septic TKR. While there are several cytokines that distinctly separate these two cohorts, this analysis indicated that the degree of inflammation experienced between these groups is comparable. This finding agrees with the clinical decision to address inflammation and perform revision surgery, and these markers (IL-10, IL-12p70, IL-13, IL-17A, IL-4, and TNF- $\alpha$ ) may show promise as helpful diagnostic monitoring markers for patients suffering from inflammatory complications in the absence of infection.

While this study had several limitations (i.e., single operating surgeon, heterogeneous cohort of patients, pathogen variability), it represents a novel characterization of tissue-level cytokines across different anatomical locations in response to implants as well as infection-specific inflammation. These cytokines may give insight into the health of localized tissue following these procedures, and the results highlight the utility of investigating a localized view of tissue health by testing tissues surrounding the joint following these procedures. At the time of publication, all patients had reached at least the one-year post-operative follow up without need for revision with no recurrent infections, and the predictive value of these cytokines for successful surgical outcomes is of interest in future studies. These cytokines could potentially be incorporated to intraoperatively assess the degree of inflammation during surgery, providing information in real time about the viability of tissues for debridement. A more focused investigation of infection-specific markers IL-1 $\alpha$, IL-1 $\beta$, IL-6, IL-8, MCP-1, MIP-1 $\alpha$, and MIP-1 $\beta$ could provide insight into the power of these cytokines to discriminate aseptic vs. septic tissues. 


\subsection{Conclusions}

In conclusion, this exploratory pilot study identified several cytokines that exhibited higher concentrations in response to implant-related and infection-specific post-operative inflammation. Some of these cytokines have been previously implicated in chronic inflammation and infection following TKA and TKR on a systemic level [11, 12, 30-33], and this study confirmed this trend on a localized tissue level and identified implant-related and infection-specific tissue-level cytokines. Previous studies have already illustrated that local inflammation is much more important for early post-operative recovery for a few markers [6], and this study expanded on that knowledge to provide an extended view of inflammatory cytokines involved in tissue health. Additionally, spatially dependent responses in cytokine concentrations were observed for IL-1 $\alpha$ and IL-1 $\beta$ when both implant and infection were present, indicating that proximity to infection is important in the response to PJI. Future work will focus on understanding the upstream and downstream factors associated with cytokine response in these chronic inflammatory scenarios.

Overall, investigating the localized tissue-level cytokines to understand implant-related and infection-specific inflammatory complications following knee arthroplasty offered insight into localized response and the disparities between septic and aseptic inflammation in these surgical scenarios. Although this study did not control for age, sex, or BMI, these cytokines were not significantly correlated to these variables, suggesting these were not confounding factors (Table 2)

in this study. Future work will include a larger cohort of patients to control for these factors and other comorbidities. Ultimately, this study provided a basis to study these cytokines in surgical scenarios as a quantitative means to understand localized tissue health for debridement. 


\subsection{Acknowledgements}

Funding for this work was provided by the National Institutes of Health [K08AR073921], and institutional support provided by the West Virginia University School of Medicine, Department of Orthopaedics. This work could not have been completed without the efforts of Jennifer Eicher in recruitment of patients for the study, Suzanne Danley and her critical editorial suggestions, and Clara Warner for graphic design images of the knee anatomy.

\subsection{References}

[1] Schulte W, Bernhagen J, Bucala R. Cytokines in sepsis: potent immunoregulators and potential therapeutic targets--an updated view. Mediators Inflamm. 2013;2013:165974. doi: $10.1155 / 2013 / 165974$

[2] Dinarello CA. Historical insights into cytokines. Eur J Immunol. Nov 2007;37 Suppl 1:S3445. doi:10.1002/eji.200737772

[3] Turner MD, Nedjai B, Hurst T, Pennington DJ. Cytokines and chemokines: At the crossroads of cell signalling and inflammatory disease. Biochim Biophys Acta. Nov 2014;1843(11):25632582. doi:10.1016/j.bbamcr.2014.05.014

[4] Miller RE, Miller RJ, Malfait AM. Osteoarthritis joint pain: the cytokine connection. Cytokine. Dec 2014;70(2):185-93. doi:10.1016/j.cyto.2014.06.019

[5] Lisowska B, Maśliński W, Małdyk P, Zabek J, Baranowska E. The role of cytokines in inflammatory response after total knee arthroplasty in patients with rheumatoid arthritis. Rheumatol Int. May 2008;28(7):667-71. doi:10.1007/s00296-007-0508-1 
[6] Ugraş AA, Kural C, Kural A, Demirez F, Koldaş M, Cetinus E. Which is more important after total knee arthroplasty: Local inflammatory response or systemic inflammatory response? Knee. Mar 2011;18(2):113-6. doi:10.1016/j.knee.2010.03.004

[7] Rodriguez A, Meyerson H, Anderson JM. Quantitative in vivo cytokine analysis at synthetic biomaterial implant sites. J Biomed Mater Res A. Apr 2009;89(1):152-9. doi:10.1002/jbm.a.31939 [8] Si HB, Yang TM, Zeng Y, et al. Correlations between inflammatory cytokines, muscle damage markers and acute postoperative pain following primary total knee arthroplasty. BMC Musculoskelet Disord. Jun 2017;18(1):265. doi:10.1186/s12891-017-1597-y

[9] Shah K, Mohammed A, Patil S, McFadyen A, Meek RM. Circulating cytokines after hip and knee arthroplasty: a preliminary study. Clin Orthop Relat Res. Apr 2009;467(4):946-51. doi:10.1007/s11999-008-0562-3

[10] Ackerman IN, Bohensky MA, Zomer E, et al. The projected burden of primary total knee and hip replacement for osteoarthritis in Australia to the year 2030. BMC Musculoskelet Disord. Feb 2019;20(1):90. doi:10.1186/s12891-019-2411-9

[11] Wylde V, Beswick A, Bruce J, Blom A, Howells N, Gooberman-Hill R. Chronic pain after total knee arthroplasty. EFORT Open Rev. Aug 2018;3(8):461-470. doi:10.1302/20585241.3 .180004

[12] Lim HA, Song EK, Seon JK, Park KS, Shin YJ, Yang HY. Causes of Aseptic Persistent Pain after Total Knee Arthroplasty. Clin Orthop Surg. Mar 2017;9(1):50-56. doi:10.4055/cios.2017.9.1.50

[13] Parvizi J, Gehrke T, Infection ICGoPJ. Definition of periprosthetic joint infection. J Arthroplasty. Jul 2014;29(7):1331. doi:10.1016/j.arth.2014.03.009 
[14] Tande AJ, Patel R. Prosthetic joint infection. Clin Microbiol Rev. Apr 2014;27(2):302-45. doi:10.1128/CMR.00111-13

[15] Li C, Renz N, Trampuz A. Management of Periprosthetic Joint Infection. Hip Pelvis. Sep 2018;30(3):138-146. doi:10.5371/hp.2018.30.3.138

[16] Currie HN, Loos MS, Vrana JA, Dragan K, Boyd JW. Spatial cytokine distribution following traumatic injury. Cytokine. Apr 2014;66(2):112-8. doi:10.1016/j.cyto.2014.01.001

[17] Han AA, Currie HN, Loos MS, et al. Spatiotemporal phosphoprotein distribution and associated cytokine response of a traumatic injury. Cytokine. Mar 2016;79:12-22. doi:10.1016/j.cyto.2015.12.006

[18] Hauser CJ, Zhou X, Joshi P, et al. The immune microenvironment of human fracture/softtissue hematomas and its relationship to systemic immunity. J Trauma. May 1997;42(5):895-903; discussion 903-4. doi:10.1097/00005373-199705000-00021

[19] Hayden FG, Fritz R, Lobo MC, Alvord W, Strober W, Straus SE. Local and systemic cytokine responses during experimental human influenza A virus infection. Relation to symptom formation and host defense. J Clin Invest. Feb 1998;101(3):643-9. doi:10.1172/JCI1355

[20] Cuingnet R, Rosso C, Chupin M, et al. Spatial regularization of SVM for the detection of diffusion alterations associated with stroke outcome. Med Image Anal. Oct 2011;15(5):729-37. doi:10.1016/j.media.2011.05.007

[21] Clough GF, Jackson CL, Lee JJ, Jamal SC, Church MK. What can microdialysis tell us about the temporal and spatial generation of cytokines in allergen-induced responses in human skin in vivo? J Invest Dermatol. Dec 2007;127(12):2799-806. doi:10.1038/sj.jid.5700930

[22] Postler A, Lützner C, Beyer F, Tille E, Lützner J. Analysis of Total Knee Arthroplasty revision causes. BMC Musculoskelet Disord. 02 2018;19(1):55. doi:10.1186/s12891-018-1977-y 
[23] Imamura M, Ezquerro F, Marcon Alfieri F, et al. Serum levels of proinflammatory cytokines in painful knee osteoarthritis and sensitization. Int $\mathbf{J}$ Inflam. 2015;2015:329792. doi:10.1155/2015/329792

[24] Usher KM, Zhu S, Mavropalias G, Carrino JA, Zhao J, Xu J. Pathological mechanisms and therapeutic outlooks for arthrofibrosis. Bone Res. 2019;7:9. doi:10.1038/s41413-019-0047-x

[25] Arango Duque G, Descoteaux A. Macrophage cytokines: involvement in immunity and infectious diseases. Front Immunol. 2014;5:491. doi:10.3389/fimmu.2014.00491

[26] Chaudhry H, Zhou J, Zhong Y, et al. Role of cytokines as a double-edged sword in sepsis. In Vivo. 2013 Nov-Dec 2013;27(6):669-84.

[27] Cavaillon JM, Adib-Conquy M, Fitting C, Adrie C, Payen D. Cytokine cascade in sepsis. Scand J Infect Dis. 2003;35(9):535-44. doi:10.1080/00365540310015935

[28] Ma L, Zhang H, Yin YL, et al. Role of interleukin-6 to differentiate sepsis from noninfectious systemic inflammatory response syndrome. Cytokine. 12 2016;88:126-135. doi:10.1016/j.cyto.2016.08.033

[29] Shahzad A, Knapp M, Lang I, Köhler G. Interleukin 8 (IL-8) - a universal biomarker? Int Arch Med. Jun 2010;3:11. doi:10.1186/1755-7682-3-11

[30] Delanois RE, Mistry JB, Gwam CU, Mohamed NS, Choksi US, Mont MA. Current Epidemiology of Revision Total Knee Arthroplasty in the United States. J Arthroplasty. 09 2017;32(9):2663-2668. doi:10.1016/j.arth.2017.03.066

[31] Saleh A, George J, Faour M, Klika AK, Higuera CA. Serum biomarkers in periprosthetic joint infections. Bone Joint Res. Jan 2018;7(1):85-93. doi:10.1302/2046-3758.71.BJR-2017-0323

[32] Frangiamore SJ, Siqueira MB, Saleh A, Daly T, Higuera CA, Barsoum WK. Synovial Cytokines and the MSIS Criteria Are Not Useful for Determining Infection Resolution After 
Periprosthetic Joint Infection Explantation. Clin Orthop Relat Res. Jul 2016;474(7):1630-9. doi:10.1007/s11999-016-4710-x

[33] Rider P, Carmi Y, Cohen I. Biologics for Targeting Inflammatory Cytokines, Clinical Uses, and Limitations. Int J Cell Biol. 2016;2016:9259646. doi:10.1155/2016/9259646

[34] Hulse RE, Kunkler PE, Fedynyshyn JP, Kraig RP. Optimization of multiplexed bead-based cytokine immunoassays for rat serum and brain tissue. J Neurosci Methods. Jun 2004;136(1):8798. doi:10.1016/j.jneumeth.2003.12.023

[35] Schäfer J, Strimmer K. A shrinkage approach to large-scale covariance matrix estimation and implications for functional genomics. Stat Appl Genet Mol Biol. 2005;4:Article32. doi:10.2202/1544-6115.1175

[36] Bland JM, Altman DG. Multiple significance tests: the Bonferroni method. BMJ. Jan 1995;310(6973):170. doi:10.1136/bmj.310.6973.170

[37] Obanda V, Omondi GP, Chiyo PI. The influence of body mass index, age and sex on inflammatory disease risk in semi-captive Chimpanzees. PLoS One. 2014;9(8):e104602. doi:10.1371/journal.pone.0104602

[38] Frasca D, Blomberg BB, Paganelli R. Aging, Obesity, and Inflammatory Age-Related Diseases. Front Immunol. 2017;8:1745. doi:10.3389/fimmu.2017.01745

[39] Vendrell J, Broch M, Vilarrasa N, et al. Resistin, adiponectin, ghrelin, leptin, and proinflammatory cytokines: relationships in obesity. Obes Res. Jun 2004;12(6):962-71. doi:10.1038/oby.2004.118

[40] Lee YS, Koo KH, Kim HJ, et al. Synovial Fluid Biomarkers for the Diagnosis of Periprosthetic Joint Infection: A Systematic Review and Meta-Analysis. J Bone Joint Surg Am. Dec 2017;99(24):2077-2084. doi:10.2106/JBJS.17.00123 
[41] Shahi A, Parvizi J. The role of biomarkers in the diagnosis of periprosthetic joint infection. EFORT Open Rev. Jul 2016;1(7):275-278. doi:10.1302/2058-5241.1.160019

[42] Chen MF, Chang CH, Yang LY, et al. Synovial fluid interleukin-16, interleukin-18, and CRELD2 as novel biomarkers of prosthetic joint infections. Bone Joint Res. Apr 2019;8(4):179188. doi:10.1302/2046-3758.84.BJR-2018-0291.R1

[43] Fassbender K, Dempfle CE, Mielke O, et al. Proinflammatory cytokines: indicators of infection in high-risk patients. J Lab Clin Med. Nov 1997;130(5):535-9. doi:10.1016/s00222143(97)90131-1

[44] Matsumoto H, Ogura H, Shimizu K, et al. The clinical importance of a cytokine network in the acute phase of sepsis. Sci Rep. 09 2018;8(1):13995. doi:10.1038/s41598-018-32275-8 [45] Ramnath RD, Weing $\mathrm{S}$, He $\mathrm{M}$, et al. Inflammatory mediators in sepsis: Cytokines, chemokines, adhesion molecules and gases. Journal of Organ Dysfunction. 2009;2(2):80-92. doi:10.1080/17471060500435662

[46] Ye Q, Shao WX, Xu XJ, Yang YZ. The clinical application value of cytokines in treating infectious diseases. PLoS One. 2014;9(6):e98745. doi:10.1371/journal.pone.0098745

[47] Monastero RN, Pentyala S. Cytokines as Biomarkers and Their Respective Clinical Cutoff Levels. Int J Inflam. 2017;2017:4309485. doi:10.1155/2017/4309485

[48] Tanaka T, Narazaki M, Kishimoto T. IL-6 in inflammation, immunity, and disease. Cold Spring Harb Perspect Biol. Sep 2014;6(10):a016295. doi:10.1101/cshperspect.a016295

[49] Bergsbaken T, Fink SL, Cookson BT. Pyroptosis: host cell death and inflammation. Nat Rev Microbiol. Feb 2009;7(2):99-109. doi:10.1038/nrmicro2070

[50] Trumpfheller C, Tenner-Racz K, Racz P, Fleischer B, Frosch S. Expression of macrophage inflammatory protein (MIP)-1alpha, MIP-1beta, and RANTES genes in lymph nodes from HIV+ 
individuals: correlation with a Th1-type cytokine response. Clin Exp Immunol. Apr 1998;112(1):92-9. doi:10.1046/j.1365-2249.1998.00555.x

[51] Ruiz Silva M, van der Ende-Metselaar H, Mulder HL, Smit JM, Rodenhuis-Zybert IA. Mechanism and role of MCP-1 upregulation upon chikungunya virus infection in human peripheral blood mononuclear cells. Sci Rep. 08 2016;6:32288. doi:10.1038/srep32288 


\section{Chapter 3}

Tissue-Level Cytokines in a Rodent Model of Chronic ImplantAssociated Inflammation and Infection 


\section{Tissue-Level Cytokines in a Rodent Model of Implant-Associated Inflammation and Infection $^{2}$}

Systemic cytokine concentrations have been extensively studied in implant-associated infections, providing sensitive diagnostic markers. However, less is known about the relationships of tissue-level cytokines surrounding the joint. The aim of this study was to define the cytokine profiles of tissues to investigate the use of these cytokines as markers of debridement in chronic joint infection. Using a rodent model, muscle samples were obtained from rats following Kirschner wire implantation and infection with Staphylococcus aureus to determine if: 1) differences exist in cytokine concentrations with proximity to infection, and 2) localized infection-specific markers can be identified on a tissue level to potentially serve as debridement markers in the future. Samples were collected from 4 distinct locations, and the concentrations of IL-1 $\alpha$, IL-1 $\beta$, IL-4, IL5, IL-6, IL-10, IL-12p70, IL-13, GM-CSF, IFN- $\gamma$, and TNF- $\alpha$ were quantified in each sample, relative to the amount of tissue. Cytokine concentrations differed with proximity to the joint when implant or infection was present, and tissues at the operative knee joint showed the highest levels of most cytokines. Additionally, IL-1 $\beta$, IL-4, and IL-6 showed promise, beyond diagnostics, as tissue-level indicators of infection response. Ultimately, this study illustrated that tissue-level evaluation provided insight into infection-specific response, and these markers may be useful for guiding debridement of implant-associated infections.

\footnotetext{
2 Parts of this chapter have been published previously from Prince N, Penatzer JA, Shackleford TL, Stewart, EK, Dietz, MJ, and Boyd, JW. Tissue-Level Cytokines in a Rodent Model of Implant-Associated Infection. Journal of Orthopaedic Research. 9, 167 (2020). Reproduced with permission from Wiley.
} 


\subsection{Introduction}

Implant-associated infections are a post-operative complication following total knee arthroplasty (TKA) procedures, affecting tens of thousands of patients per year in the United States [1]. Prosthesis-related infections, such as periprosthetic joint infection (PJI), affect the implant and surrounding tissues, and they can become chronic if bacteria form a biofilm on the prosthesis, creating a barrier against host response and antibiotic treatment [2]. This pressure on the host's immune system combined with antibiotic resistance of the bacteria makes these infections difficult to reconcile and often results in high failure rates for treatment. Failure rates for all TKA procedures remain around 5\% but are much higher with infected joints, with reported failure rates ranging from $14-25 \%[3-5]$.

Early diagnosis of infection is critical, and a range of sensitive and specific biomarkers, including C-reactive protein (CRP), alpha-defensin, D-dimer, as well as cytokines like interleukin (IL)-1 $\beta$, and IL-6, have been highlighted for their diagnostic utility in implant-associated infections like PJI [6-9]. Cultures, biopsies, and imaging techniques like PET-MRI and PET-CT have also been utilized with high sensitivity [10]. Prompt diagnosis can lead to better outcomes in surgical treatment, and serum biomarkers are most often used to aid diagnostic accuracy [11]. CRP is the most common diagnostic marker for implant infections, as it is both inexpensive and rapid [12], however, it suffers from low specificity. CRP levels can be high in a wide range of inflammatory processes, including the healing process following TKA procedures [13], so a high CRP level alone cannot positively confirm presence of infection. More recently, diagnostic efforts have shifted towards the identification and validation of cytokine markers. 
The cytokine response to infection involves both pro- and anti-inflammatory cytokines that are recruited to the site [14-16]. Due to cytokines' central roles in infection response, recent studies have investigated cytokine measurements for their diagnostic utility, especially in infections due to Staphylococcus aureus, the most common pathogen implicated in implant-associated infections. Pro-inflammatory cytokines IL-6, IL-1 $\beta$, and tumor necrosis factor alpha (TNF- $\alpha$ ) were first analyzed in synovial fluid of total hip arthroplasty (THA) patients and showed the ability to discriminate between aseptic and septic joints [17]. Since then, a variety of other cytokines, including interferon gamma (IFN- $\gamma$ ) [18], IL-4 [19], and IL-10 have also been pursued [20]. Frangiamore et al. identified IL-6 and IL-1 $\beta$ not only as markers with excellent diagnostic strength, but also noted their efficacy for monitoring response to infection treatment in synovial fluid [18]. While these cytokines have shown increased sensitivity and specificity for diagnosis in serum and synovial fluid, they have not been evaluated to expand their use beyond diagnostics into indicators of inflammatory immune response to aid treatment.

Surgical debridement is utilized to treat chronic implant infections, but it relies heavily on subjective assessment of the state of the tissues surrounding the joint [21]. Understanding local tissue health is important for debridement of infected joints, as surgeons must delineate healthy from non-healthy tissue to promote infection resolution [22]. Complete removal of infected tissue is critical, as re-infection only requires a small number of bacteria [23]. Discerning healthy tissue from infected tissue can be challenging for clinicians, and insufficient debridement is a possible source for failure of treatment [24]. However, tissues are not intra-operatively analyzed beyond identification of the "4 C's"- Color, Consistency, Contractility, and Capacity to bleed- and quantification of white blood cells. Rarely, the presence or absence of bacteria via culture or 
polymerase chain reaction (PCR) is utilized [17, 25]. Intra-operative assessment of tissues surrounding the joint for inflammatory cytokine markers could reveal important insights into tissue health, as these biomarkers are intimately related to infection response $[6-8,26]$ and wound resolution [27]. Further, identification of markers to guide debridement would benefit clinical decision making and allow surgeons to quantitatively assess the state of tissue in infected joints. Cytokine biomarkers that have previously been utilized for their roles in diagnostics may provide an avenue to pursue quantitative markers of debridement margins. Defining the tissue-level concentrations is the first step to evaluating the ability of cytokines as markers of healthy and nonhealthy tissue in chronic implant-associated joint infection.

The purpose of this study was to assess tissue-level cytokine responses in a rodent model of chronic implant-associated infection, specifically with the purpose of highlighting new biomarkers of debridement for PJI. Cytokine concentrations were measured in skeletal muscle samples taken from Sprague-Dawley rats implanted with a Kirschner wire (K-wire) and exposed to S. aureus in the joint cavity. Levels of pro-inflammatory and anti-inflammatory cytokines were measured for the following targets: IL-1 $\alpha$, IL-1 $\beta$, IL-4, IL-5, IL-6, IL-10, IL-12p70, IL-13, IFN- $\gamma$, TNF- $\alpha$, and granulocyte-macrophage colony-stimulating factor (GM-CSF). These factors were specifically chosen because of their use as diagnostic markers [28], roles in tissue healing [27], and the ability to give insight into overall tissue health [29]. Ultimately, this study aimed to expand on the diagnostic roles of these cytokines and investigate them as markers of debridement margins. 


\subsection{Materials and methods}

\subsubsection{Animals}

All procedures were performed under the guidelines approved by the West Virginia University Animal Care and Use Committee (Approval \#1803013294). Adult male and female Sprague-Dawley rats (Charles River, Wilmington, MA) were housed individually with a 12:12 light/dark cycle and ad libitum access to standard chow and water. Animals were divided into 3 groups: Sham (N=6), Implant Only (N=6), and Infection+Implant (Inf+Implant; N=6). Animal numbers for each group were obtained by performing a statistical power analysis using an alpha value of 0.05 and a difference to detect of 2 . The power analysis was based on previous work to detect trauma-related cytokines, specifically IL-1 $\beta$ [30]. The power analysis necessitated a group size of $\mathrm{N}=6$ when including both male and female rodents. All procedures were based on previous models of implant-associated infections in rats with K-wire implant [31-35], and tissues and blood were collected from all animals 21 days post-surgery.

\subsubsection{Experimental rat model of chronic localized infection}

Chronic joint infection was established based on literature protocols and previous work. Briefly, after adequate isoflurane anesthesia, the right leg was shaved and prepared for surgery. Under sterile conditions, the knee joint was exposed, and a hole was drilled into the medullary cavity of the femur. For Implant Only and Inf+Implant groups, a 3-cm (male) or 2-cm (female) by 1-mm stainless steel K-wire was implanted into the bone. For Inf+Implant groups, $20 \mu \mathrm{L}$ of $1.8 \times 10^{7} \mathrm{CFU} / \mathrm{mL}$ suspension of Methicillin-sensitive Staphylococcus aureus (MSSA; ATCC 25923, clinical isolate [Manassas, VA]) was injected into the medullary cavity after insertion of K-wire. This dose was chosen based on previous models to create a chronic localized infection but 
to avoid systemic sepsis [36]. Additionally, a previous study found that inflammation due to surgery can persist until the 21-day time point, so this time point was chosen for tissue collection to optimize the measurement of infection-specific inflammatory cytokine responses [36]. Body temperature was monitored throughout the 21-day time period for presence of fever to ensure no systemic sepsis occurred. For all groups, the exposed joint was closed with 3-0 vicryl (Ethicon,

Inc., Somerville, NJ) and staples, then Vetbond tissue adhesive (3M, St. Paul, MN) was applied externally. Buprenorphine SR (ZooPharm, Fort Collins, CO) was administered subcutaneously as an analgesic providing 72-hour pain relief [37]. Following closure of the knee joint, X-rays were taken at the 21-day time point and graded according to Aktekin et al [38]. Detailed methods and results for X-rays can be found in Appendix B.

\subsubsection{Tissue collection}

At 21-days post-surgery, the animals were again placed under anesthesia, as described above, and tissue and blood samples were collected. The 21-day post-surgery time point was chosen for sample collection as it allowed for the local effects in bone and soft tissue $[31,33,36]$. Once samples were collected, the animals were euthanized under isoflurane anesthesia with cardiac puncture, and one cc of Euthasol (Patterson Veterinary, Greenly, CO) was administered. Blood was collected in BD Vacutainer SST collection tubes (Becton Dickinson Vacutainer, Franklin Lakes, NJ), and serum was separated per manufacturer's instructions. Blood samples were tested for white blood cell count (WBC) and red blood cell count (RBC) via standard medical lab testing; CRP (Invitrogen, Carlsbad, CA) and IL-6 levels (Invitrogen) were tested via enzymelinked immunosorbent assay (ELISA) in serum. These systemic measurement parameters can be found in Table 3.1. For tissue collection, four tissue locations were gently dissected from fascial 
attachments, and skeletal muscle was collected. These locations were chosen to compare tissuelevel cytokines directly at the infected joint to nearby locations to evaluate infection-specific tissue responses across spatial gradients. Muscle samples were harvested from the following four locations: at the operative knee joint; $1.5 \pm 0.2 \mathrm{~cm}$ proximal from operative knee joint; $1.0 \pm 0.2 \mathrm{~cm}$ distal from operative knee joint; and from the contralateral leg knee joint. Figure 3.1 illustrates the sampling locations, labeled as A-D. Tissue samples were cultured to confirm presence or absence of S. aureus infection at 21 days post-surgery using established methods [39]. Bacterial load at the operative knee location for the three groups can be found in Table 3.1.

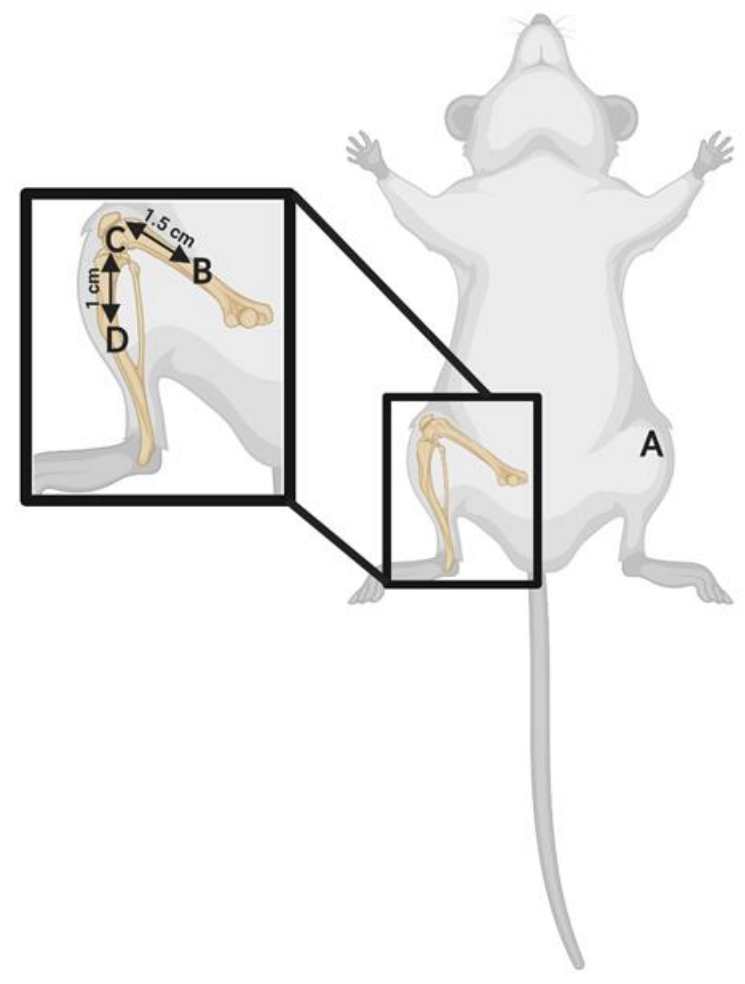

Figure 3. 1. Sampling locations for muscle samples collected at 21 days post-surgery. Immediately prior to euthanasia, tissue samples were collected by gently dissecting skeletal muscle from fascial attachments. Four tissue locations were taken, denoted A-D. A) Contralateral leg knee joint; B) Operative leg, $1.5 \mathrm{~cm}$ proximal from knee joint; C) Operative leg knee joint; D) Operative leg, 1.0 distal from knee joint. 


\subsubsection{Sample preparation}

Samples were washed immediately with $1 \mathrm{X}$ cold phosphate-buffered saline (PBS) to remove blood and debris. Tissues were grossly dissected using a scalpel to remove scar and connective tissue, then stored at $-80^{\circ} \mathrm{C}$. Samples were ground cryogenically and lyophilized for 24 hours. For analysis, lyophilized tissue was thawed for $10 \mathrm{~min}$ at $4^{\circ} \mathrm{C}$ in $1 \mathrm{~mL}$ of cell lysis buffer (Bio-Rad, Hercules, CA) containing $20 \mathrm{mM}$ phenylmethylsulfonyl fluoride (Sigma-Aldrich, St. Louis, MO). Protein extraction was performed using methods adapted from Hulse et al [40]. Thawed samples were vortexed for $1-3$ seconds and centrifuged at 5,000 x g for 5 minutes at $4^{\circ} \mathrm{C}$. The supernatant was collected and tested for total protein content using a Pierce BCA Protein Assay Kit (Thermo Scientific, Waltham, MA), according to manufacturer's instructions. Absorbance values for total protein content were determined on an Infinite M1000 multimode plate reader (Tecan, Raleigh, NC).

\subsubsection{Cytokine and phosphoprotein measurement}

To standardize samples for total protein content, tissue homogenates were individually diluted to a total protein concentration of $900 \mu \mathrm{g} / \mathrm{mL}$ with cell lysis buffer (Bio-Rad). Cytokine quantification was performed using a magnetic bead-based multiplex Rat Cytokine Th1/Th2 Kit (Bio-Rad) and measured using a Bio-Plex 200 suspension array system and Pro II Wash Station (Bio-Rad), according to the manufacturer's instructions. The Th1/Th2 kit included the following cytokines: IL-1 $\alpha$, IL-1 $\beta$, IL-4, IL-5, IL-6, IL-10, IL-12p70, IL-13, GM-CSF, IFN- $\gamma$, and TNF- $\alpha$. Results for these 11 cytokines were included in this analysis. Additionally, a total of 20 phosphoproteins related to wound healing were tested via custom Bio-Rad multiplex phosphoprotein ELISA panels. The following targets were assessed, and sites of phosphorylation 
are indicated in parenthesis. CREB (S133), HSP27 (S78), IкB- $\alpha$ (S32/S36), MEK1 (S217/S221), RPS6 (S235/S236), Smad2 (S165/S167), Src (Y416), Syk (Y352), c-Jun (S63), AKT (S473), p53 (S15), p38 (Y180/Y182), p70S6K (T389), PTEN (S380), ZAP-70 (Y319), BAD (S136), ERK1/2 (T202/Y204 T185/Y187), p90RSK (S380), VEGFR2 (Y1175), and NF-kB p65 (S536). Phosphoprotein results are discussed following the discussion of cytokines in section 3.4.

\subsubsection{Statistical analysis}

Data were analyzed using Prism 5 (GraphPad, San Diego, CA) and SAS JMP (Cary, NC). Standard curves for cytokine concentrations were generated for each protein using either a four(4PL) or five-parameter logistic (5PL) regression model, depending on individual protein. Cytokine concentrations were determined using standard curve interpolation, then corrected by dilution factor. To compare cytokines, these values were standardized by tissue weight and converted to nanograms of cytokine per gram of tissue (ng/g). Implant and Inf+Implant groups were analyzed with respect to relative fluorescence compared to Sham group. Samples with fluorescence intensity values below the lower limit of quantitation (LLOQ) or above the upper limit of quantitation (ULOQ) were omitted from statistical comparisons. Outliers were identified using the $1.5 \mathrm{X}$ interquartile range (IQR) rule and omitted from analysis. Two-way analysis of variance (ANOVA) with Bonferroni's post-test was used to determine significant differences between Sham $(\mathrm{N}=6)$, Implant Only $(\mathrm{N}=6)$, and $\mathrm{Inf}+\operatorname{Implant}(\mathrm{N}=6)$ groups as well as between locations (i.e., in the Sham group, operative knee joint vs. operative leg proximal). Each tissue homogenate was tested in duplicate for cytokine concentration. Data are expressed as the mean \pm standard error of the mean (SEM). 
Table 3. 1. Clinically-Relevant Parameters at 21 Days Post-Surgery. At 21 days post-surgery, blood was collected and tested for RBC and WBC; IL-6 and CRP were measured in rat serum. Additionally, skeletal muscle samples were cultured to calculate bacterial load at the joint. RBC are shown in scientific notation as million(s) per microliter of blood; similarly, WBC are shown as thousand(s) per microliter of blood. CRP is shown in milligrams of CRP per liter of serum, and IL-6 is shown as picograms of protein per milliliter of serum. Bacterial load is shown as colonyforming units (CFUs) per gram of tissue. Significance at $p<0.05$ is denoted with superscripts " $S$ " for different than Sham, "C" for different than Implant Only, and "I" for different than Inf+Implant. Comparisons between groups were identified by one-way ANOVA with Tukey's post-test. N.D. is an abbreviation for "not detected."

\begin{tabular}{|l|l|l|l|}
\hline & \multicolumn{1}{|c|}{ SHAM } & \multicolumn{1}{|c|}{ IMPLANT ONLY } & \multicolumn{1}{c|}{ INF+IMPLANT } \\
\hline RBC $\left(\mathrm{x} 10^{6} / \mu \mathrm{L}\right)$ & $8.14 \pm 0.53$ & $7.98 \pm 0.54$ & $7.69 \pm 0.60$ \\
\hline WBC $\left(\mathrm{x} 10^{3} / \mu \mathrm{L}\right)$ & $3.97 \pm 0.78$ & $2.90 \pm 2.14$ & $3.63 \pm 1.40$ \\
\hline CRP $(\mathrm{mg} / \mathrm{L})$ & $428.51 \pm 164.92$ & $620.24 \pm 296.11$ & $789.39 \pm 181.20$ \\
\hline \begin{tabular}{l} 
IL-6 $(\mathrm{pg} / \mathrm{mL})$ \\
\hline $\begin{array}{l}\text { Bacterial load } \\
(\mathrm{CFU} / \mathrm{g})^{*}\end{array}$
\end{tabular} & $24.50 \pm 0.69^{\mathrm{C}, \mathrm{I}}$ & $68.18 \pm 15.94^{\mathrm{S}}$ & $118.06 \pm 13.50^{\mathrm{S}}$ \\
\hline
\end{tabular}

*Note: Bacterial load was calculated in tissue cultures, while all other parameters in Table 3.1 were measured in serum.

\subsection{Cytokine Analysis of Rodent Tissues Between Sham, Implant Only, and Inf+Implant Groups}

Changes in tissue-level cytokine concentrations were observed between Sham, Implant Only, and Inf+Implant groups in implant-related and infection-specific manners. Overall, cytokine concentrations were higher for Implant Only and Inf+Implant groups compared to Sham. Intragroup differences between locations were also investigated. Tissues at the operative knee (i.e., the site of debridement) were compared to all other locations. Cytokine profiles were compared 
between these locations to look for spatially-dependent differences relevant for debridement. The operative knee and operative leg distal locations generally produced higher cytokine concentrations than the contralateral knee and operative leg proximal locations. The specific results are discussed over the following sections and can be seen in Figures 3.2-3.4.

\subsubsection{Eight cytokines elevated in implant-related manner}

Of the eleven cytokines, eight produced increases in both Implant Only and Inf+Implant groups compared to Sham (Figure 3.2): IL-1 $\alpha$, IL-5, IL-10, IL-12p70, IL-13, GM-CSF, IFN- $\gamma$, and TNF- $\alpha$. These cytokines showed elevated concentrations at the operative knee and operative distal locations compared to Sham $(\mathrm{p}<0.05)$, but there were generally no significant differences between Implant Only and Inf+Implant groups (exceptions: IL-5 and IL-10 at the operative distal location). The profiles for Implant Only and Inf+Implant groups across different locations were similar for all eight of these cytokines. Group-dependent differences at $\mathrm{p}<0.05$ are marked with letters $\mathrm{S}$ (significantly different than Sham group), C (significantly different than Implant Only group), and I (significantly different than Inf+Implant group). Additionally, all tissue locations were compared to the operative knee site to understand spatial differences, and significant differences at $p<0.05$ between locations are marked with striped bars. The average values \pm SEM can be found in Appendix B. 
GM-CSF

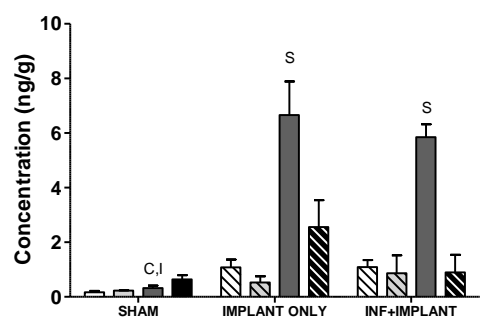

IL-10

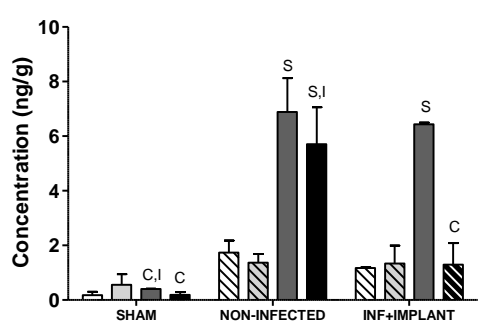

IL-13

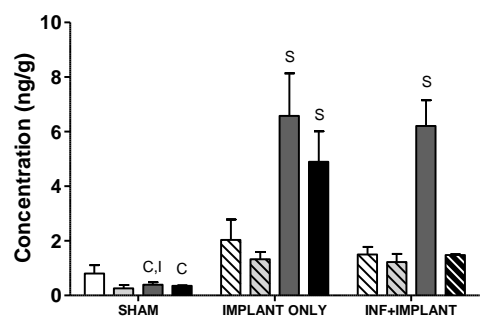

IFN- $\gamma$

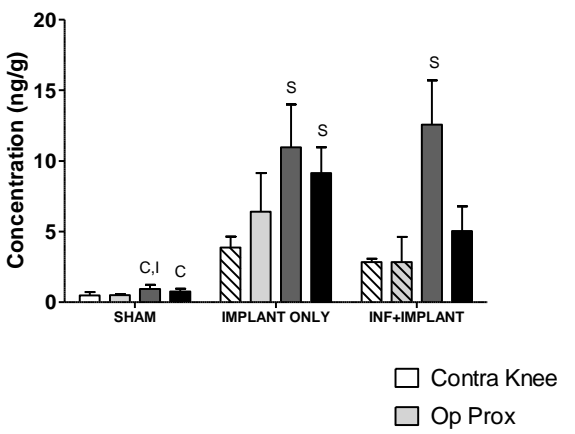

IL-5

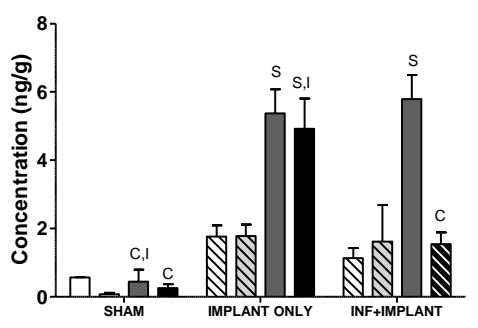

IL-12p70

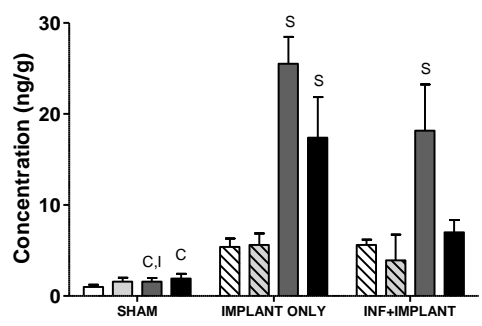

IL-1 $\alpha$

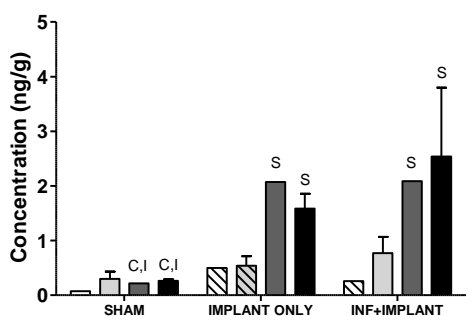

TNF- $\alpha$

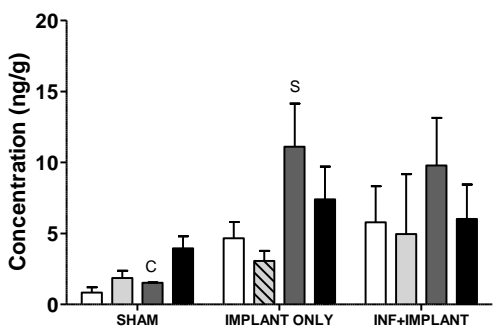

$\square$ Op Knee

Op Dist

Figure 3. 2. $I L-1 \alpha, I L-5, I L-10, I L-12 p 70, I L-13, G M-C S F, I F N-\gamma$, and TNF- $\alpha$ concentrations in response to implant and infection. Concentrations are expressed as nanogram of cytokine per gram of tissue $(\mathrm{ng} / \mathrm{g})$. Statistically significant differences $(\mathrm{p}<0.05)$ in protein concentration between Sham ( $\mathrm{N}=6)$, Implant Only $(\mathrm{N}=6)$, and $\mathrm{Inf}+\operatorname{Implant}(\mathrm{N}=6)$ groups are marked for each location: "S" represents different than Sham group; "C" denotes different than Implant Only group; "I" denotes different than Inf+Implant group. All locations were compared to the operative knee site within each group, and statistically significant differences between locations are marked with striped bars to indicate difference in concentration compared to the operative knee at $p<0.05$. 


\subsubsection{Three cytokines showed infection-specific response}

For three of the eleven cytokines, there was an increase in the Implant Only group, and a further increase in concentration was observed for the Inf+Implant group. IL-1 $\beta$, IL-4, and IL-6 showed an infection-specific trend, with the highest concentrations at the operative knee location (Fig. 3.3). The contralateral knee and operative leg proximal locations were not significantly different between Sham, Implant Only, and Inf+Implant groups ( $>0.05$ ). Group-dependent differences at $\mathrm{p}<0.05$ are marked with letters $\mathrm{S}$ (significantly different than Sham group), C (significantly different than Implant Only group), and I (significantly different than Inf+Implant group) in Figure 3.3. Additionally, all tissue locations were compared to the operative knee site, and significant differences at $\mathrm{p}<0.05$ between locations are marked with striped bars. The average values \pm SEM can be found in Appendix B. 
IL-1 $\beta$

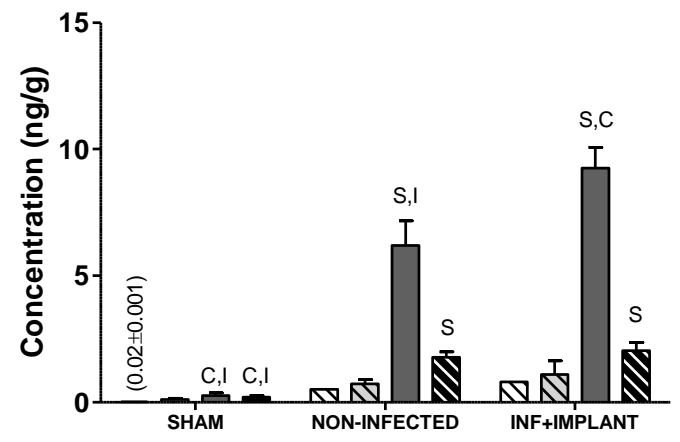

\section{IL-6}

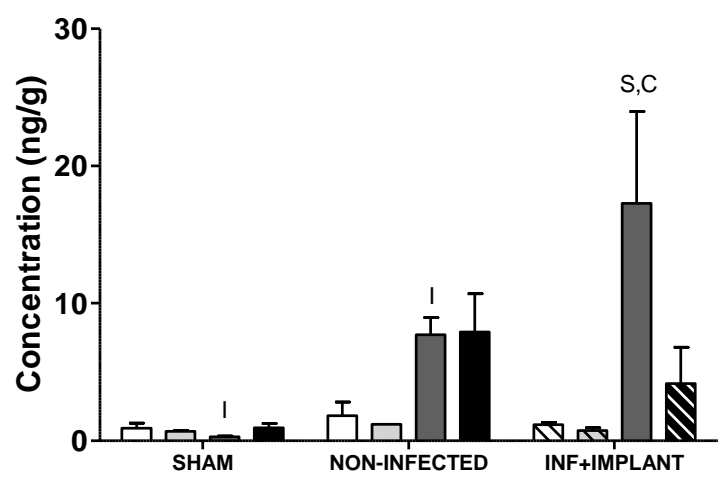

IL-4

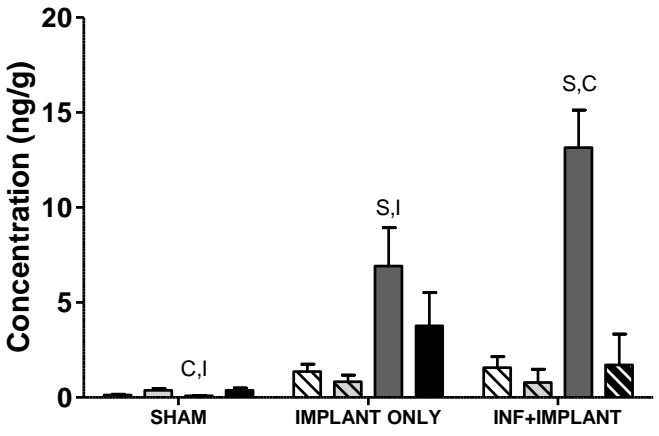

Figure 3. 3. $I L-1 \beta, I L-4$, and IL-6 tissue concentrations in response to implant and infection. Concentrations are expressed as nanogram of cytokine per gram of tissue (ng/g). Statistically significant differences $(\mathrm{p}<0.05)$ in protein concentration between Sham $(\mathrm{N}=6)$, Implant Only $(\mathrm{N}=6)$, and Inf+Implant $(\mathrm{N}=6)$ groups are marked for each location: " $\mathrm{S}$ " represents different than Sham group; "C" denotes different than Implant Only group; "I" denotes different than Inf+Implant group. All locations were compared to the operative knee site within each group, and statistically significant differences between locations are marked with striped bars to indicate the concentration at that location is different than the operative knee at $p<0.05$. All labeling is consistent with Figure 3.2. 


\subsubsection{Quadratic discriminant analysis and heat maps of cytokine profiles}

Total cytokine profiles were analyzed via QDA with SAS JMP on all measured cytokine responses at the operative knee location to compare Implant Only and Inf+Implant groups. The ANOVA data indicated that cytokine profiles were elevated in both of these groups, and QDA focused on the disparate profiles between Implant Only and Inf+Implant to identify cytokines most specific for infection on a tissue level. This analysis classified the combined concentrations of all cytokines observed into pre-determined groups (Implant Only, Inf+Implant) by plotting canonical scores calculated by the quadratic model. The 95\% confidence intervals (CIs) are shown for each group, with Implant Only in red and Inf+Implant in blue. Biplot rays are shown to denote the degree of association of each cytokine with each group. There were no misclassifications in the model. Heat maps were constructed to show relative cytokine concentrations and compare the influence of cytokines on the cumulative cytokine profile for each group. Cytokine concentrations in $\mathrm{ng} / \mathrm{g}$ were normalized between 0 (lowest value) and 1 (highest value) for each cytokine. Green represents a low relative cytokine concentration, and red represents a high relative cytokine concentration; the intensity of color denotes the degree of polarization. Relative levels in the Sham group were in the lowest $10^{\text {th }}$ percentile for all cytokines, which can be observed by the intense green color. Colors in the Implant Only and Inf+Implant ranged from the $43^{\text {rd }}$ percentile (mild green-yellow) to $100^{\text {th }}$ percentile (bright red). The heat map results complement the findings of the QDA by illustrating the contrast in relative concentrations between Implant Only and Inf+Implant groups for IL-1 $\beta$, IL-4, IL-6, and IL-12p70. 
a

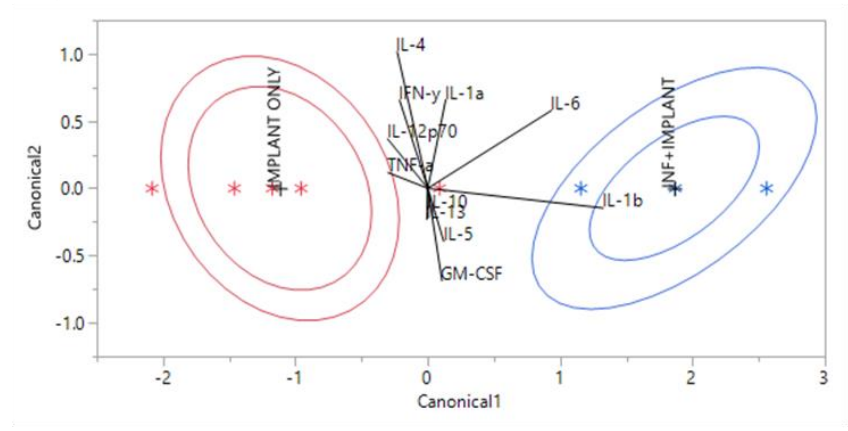

b

\begin{tabular}{|c|c|c|c|}
\hline & SHAM & IMPLANT ONLY & INF+IMPLANT \\
\hline IL-1 $\alpha$ & & & \\
\hline LL-1; & & & \\
\hline IL-4 & & & \\
\hline IL-5 & & & \\
\hline IL-6 & & & \\
\hline IL-10 & & & \\
\hline IL-12p70 & & & \\
\hline LL-13 & & & \\
\hline GMCSF & & & \\
\hline IFN- $\gamma$ & & & \\
\hline TNF- $\alpha$ & & & \\
\hline
\end{tabular}

Figure 3. 4. QDA analysis (a) and heat map of relative cytokine concentrations (b). QDA analysis (a) shows the influence of the eleven cytokines on classification into Implant Only (red) or Inf+Implant (blue) groups. Biplot rays illustrate the degree of association with each group, and ellipses are drawn to show the mean $\pm 95 \%$ CI for the cumulative cytokine profiles. Heat maps of relative cytokine concentrations (b) illustrate the relative increases in concentration between the three groups for direct comparison between cytokines. For heat maps, cytokine concentrations (ng/g) were normalized between 0 and 1 for each cytokine to show relative increases. Green represents a low relative concentration, and red represents a high relative concentration. 


\subsection{Discussion of cytokine concentrations in response to implant and infection}

Implant-associated infections like PJI are devastating post-surgical complications and are

the leading cause of implant failure in TKA procedures [1]. Diagnosis and treatment impose a significant burden for the health care system as well as individual patients, and the annual cost of infected revisions is projected to exceed $\$ 1.5$ billion by the end of 2020 [41]. Cytokine measurements in synovial fluid and serum of patients suffering from chronic PJI have illustrated high sensitivity and specificity of these targets for diagnostic purposes [20]. However, the primary method for treatment of chronic, recurrent implant-associated infections is debridement, which has highly subjective endpoints [21]. Treatment has not progressed to more objective metrics, presenting a challenge for clinicians who are tasked with precise, complete removal of infected tissues. Cytokine biomarkers currently used for diagnostics may provide potential for use as quantitative markers for establishing intra-operative debridement margins between healthy and non-healthy tissue. In this study, eleven cytokines were analyzed in a rodent model of chronic implant-associated infection to identify biomarkers that have the potential to delineate infected vs. non-infected tissues. Ultimately, these cytokines could aid clinicians in establishing debridement margins using reliable, quantitative metrics.

Eight cytokines exhibited implant-related increases in concentration at the operative knee: IL-1 $\alpha$, IL-5, IL-10, IL-12p70, IL-13, GM-CSF, IFN- $\gamma$, and TNF- $\alpha$ (Fig. 3.2). For these cytokines, Implant Only and Inf+Implant groups showed higher concentrations than the Sham group $(\mathrm{p}<0.05)$, but there were no significant differences between Implant Only and Inf+Implant groups directly at the operative knee. These cytokines have previously been linked to inflammation associated with prosthetics [42], and our results indicated that inflammation at the joint can be attributed to 
the presence of a K-wire implant (Fig. 3.2). All of these cytokines gave the highest response directly at the operative knee location, with the exception of IL-1 $\alpha$ for the Inf+Implant group, which had an unclear trend. Decreased cytokine profiles would suggest a return to healthy tissue [29], which is relevant to debridement, so all tissue locations were compared to the operative knee to investigate the spatially-disparate responses. IL- $1 \alpha$, IFN- $\gamma$, and TNF- $\alpha$ did not show the ability to reliably discriminate tissues across locations, and previous studies have noted low specificity in serum for these cytokines in implant-associated infections [43]. The ANOVA data in this study suggested they would not be reliable debridement markers (Fig. 3.2). Disparities between operative knee and operative distal sites for the Inf+Implant group were observed for IL-5, IL-10, IL-13, and IL-12p70, but these differences were not present in the Implant Only group. The Implant Only group still maintained elevated IL-5, IL-10, and IL-13 concentrations at the operative distal site (Fig. 3.2; $<<0.05$ ), but the Inf+Implant group showed a decrease at this location. These are three anti-inflammatory cytokines [44], and they all showed elevation at tissues downstream of the joint in the Implant Only group. IL-12p70 also appeared to follow this trend, but not at a statistically significant level (Fig. 3.2; p>0.05). IL-12p70 is an immunoregulatory cytokine with both pro- and anti-inflammatory functions and plays a role in cell proliferation during wound healing [45]. The exact mechanisms of cytokine dysregulation due to implant and infection are unknown, but the spatially disparate downstream effects observed for these cytokines could be due to a variety of factors, including vascular supply [46], atrophy [35], or differences in wound healing stages [47]. While outside the scope of this manuscript, the spatially disparate profiles of these cytokines due to foreign body implant warrant future investigation. GM-CSF was the only implant-related cytokine to show the expected spatially-dependent trend for both the Implant Only and Inf+Implant groups (Fig. 3.2; p<0.05). GM-CSF has been noted for its role in inflammatory autoimmune 
diseases and has shown increased concentrations in response to pro-inflammatory stimuli [48]. However, the QDA illustrated it was not a good differentiator of inflammation due to implants vs. inflammation due to infection (Fig. 3.4). Overall, the data in this study indicated that none of the implant-related cytokines were good indicators of debridement margins at the operative knee.

Infection-specific trends in cytokine responses were present for IL-1 $\beta$, IL-4, and IL-6 in this study $(\mathrm{p}<0.05)$. These cytokines have been noted for their roles in infection, demonstrating increases in serum concentrations in response to joint infection [49-51]. In this study, these cytokines showed the highest increase in response directly at the operative knee (Fig. 3.3), highlighting the importance of tissue-level disparities in response to infection. IL-1 $\beta$ and IL-6, two pro-inflammatory cytokines, have demonstrated diagnostic utility and were elevated in response to infection in this study [52]. Infection with $S$. aureus is known to trigger IL-1 $1 \beta$ release and is concomitant with cell death [53]. IL-6 is synthesized in infectious lesions to send out a warning signal of tissue damage, which is then recognized by pathogen-recognition receptors (PRRs), and it is an early initiator of infection-related inflammation [54]. Both IL-1 $\beta$ and IL-6 produce signals during infection that ultimately trigger cell death pathways [55]. For IL-1 $\beta$, all locations were significantly different than the operative knee $(\mathrm{p}<0.05)$ for both Implant Only and Inf+Implant groups (Fig. 3.3). IL-6 only reflected this trend in the Inf+Implant group, but the increased concentrations at the operative knee location compared to Implant Only suggested it is still a good indicator of infection presence ( $\mathrm{p}<0.05$, Fig. 3.3). IL-4, an anti-inflammatory cytokine, activates the Stat6 pathway in infection and suppresses cell-mediated death [56]. IL-4 trends were similar to IL-6, and only the Inf+Implant group showed a significant decrease in concentration at the operative distal location $(\mathrm{p}<0.05)$. QDA and heat maps were constructed to comparatively assess 
the contributions of these cytokines to each of the groups. The heat maps showed relative concentrations of cytokines and suggested IL-1 $1 \beta$, IL-4, and IL-6 may all serve as good indicators of infected tissues at the joint. Further analysis of QDA data indicated IL-1 $\beta$ and IL-6 are strongly indicative of Inf+Implant tissues (Fig. 3.4), while IL-4 plays a role in both Implant Only and Inf+Implant groups when considering total cytokine contributions. The QDA demonstrated that IL-1 $\beta$ and IL-6 show a stronger relationship to Inf+Implant tissues and may serve as better indicators for debridement.

The results of this study demonstrated that tissue-level concentrations of IL-1 $\beta$ and IL-6 are strongly indicative of infected tissues through ANOVA comparisons as well as QDA classifications. These cytokines have previously been validated for diagnostics of implantassociated infections in serum, and they have established roles in infection response. The findings presented here offer an opportunity to expand their utility beyond diagnostics as tissue-level indicators of infection. These results provided evidence that these cytokines are worth pursuing in the development of objective biomarkers to guide debridement and treatment of chronic implantassociated infections. Ultimately, this study laid the foundation to develop quantitative, tissuelevel biomarkers to aid surgical decision making. In the future, these cytokines could be investigated for their ability to guide intra-operative debridement. 


\subsection{Phosphoprotein and Ingenuity Pathway Analysis (IPA) to understand spatially- disparate responses}

The cytokine analysis in this study provided important insights into the tissue-level immune response to chronic infection and highlighted biomarkers that could be used for debridement of chronically infected tissues, but the analysis also provoked questions regarding the responses of tissues upstream and downstream of the operative site. The initial cytokine study primarily addressed the response directly at the operative knee, as this is the most relevant for surgical debridement, but the responses of proximal and distal tissues also showed altered concentrations of some cytokines. Increases in all cytokines, either due to implant or infection, suggested some disruption of normal inflammatory immune response (Figs. 3.2 and 3.3), and evidence suggests that excessive inflammation caused by these cytokines can delay wound healing [62]. Elevated cytokine levels at proximal or distal locations could suggest that healing processes in these tissues are compromised, so wound healing phosphoproteins were also assayed to investigate the responses proximal and distal to the operative knee.

GM-CSF, IL-4, and IL-1 $\beta$ all showed the expected trend with elevation at the operative knee, and other locations in the Implant Only and Inf+Implant groups were statistically significantly different from the operative knee at $\mathrm{p}<0.05$ (with one exception: IL-4 in the Implant Only group operative knee vs. operative distal, but it appeared to follow the trend; Figs. 3.2 and 3.3). However, the other cytokines showed elevated cytokine concentrations either at the op prox or op dist locations. Four cytokines, IL-5, IL-10, IL-12p70, and IL-13 showed elevated levels in response to implant, and the operative distal location maintained higher concentrations of these cytokines (Fig. 3.2). While IL-6, one of the infection-specific cytokines, showed significant differences between 
locations in the Inf+Implant group ( $\mathrm{p}<0.05)$, the Implant Only group did not show any significant differences between locations. Other cytokines, IL-1 $\alpha$, IFN- $\gamma$, TNF- $\alpha$ showed less spatial disparity and did not inform differential wound healing responses between locations. All of these cytokines have established roles in acute infection response [6-9, 14-16, 18-20], but chronic infections can disrupt cell signaling networks associated with these responses [63, 64]. The inflammation due to foreign body implant, infection, or both may be negatively impacting the ability of tissues to heal, but cytokines alone are not enough to understand these wound healing cascades.

To investigate the networks of tissue healing between disparate locations, phosphoprotein analysis was conducted as a follow-up to the cytokine work. Network analysis can give information about the connectivity of wound healing signaling and highlight key mediators of the response [65, 66]. QIAGEN's Ingenuity Pathway Analysis (IPA) software application is a commonly used tool for creating and analyzing complex biological networks. This method uses both experimental and literature-derived data to connect different targets, referred to as "nodes," resulting in a model of response. IPA utilizes the Ingenuity Knowledge Base, a repository derived and curated from previous studies, to identify highly interconnected "Focus Genes" and construct a network [67]. Top molecular and cellular function associated with the networks are also reported by IPA, which allows researchers to further probe into which cellular processes may be activated and which nodes are most important in the network. 


\subsection{Methods for phosphoprotein and network analysis}

\subsubsection{Statistical analysis of phosphoproteins}

Phosphoprotein data was acquired using multiplexed magnetic bead-based assays (BioRad) and measured using a Bio-Plex 200 suspension array system (Bio-Rad), as described in section 3.2.5. Data were analyzed using Prism 5 (GraphPad, San Diego, CA), and relative phosphorylation levels were calculated from fluorescence intensity values. Differences between groups and tissue locations were compared based on relative phosphorylation in samples standardized to a total protein concentration of $900 \mu \mathrm{g} / \mathrm{mL}$. Samples with fluorescence intensity values below the lower limit of quantitation (LLOQ) or above the upper limit of quantitation (ULOQ) were omitted from statistical comparisons. Outliers were identified using the 1.5 $\mathrm{X}$ interquartile range (IQR) rule and removed. Two-way analysis of variance (ANOVA) with Bonferroni's post-test was used to determine significant differences between Sham (N=6), Implant Only (N=6), and Inf+Implant ( $\mathrm{N}=6)$ groups as well as between locations (i.e., in the Sham group, operative knee joint vs. operative leg proximal). Each tissue homogenate was tested in duplicate. Data are expressed as the mean \pm standard error of the mean (SEM). ANOVAs are shown for 16 out of the total 20 phosphoproteins tested. Five phosphoproteins, HSP27 (S78), Src (Y416), p53 (S15), ZAP-70 (Y319), and NF-кB p65 (S536), did not produce statistically significant trends (data not shown).

\subsubsection{Construction of IPA networks}

Both cytokines and phosphoproteins were included in the creation of IPA networks, and all 31 targets were included (11 cytokines and 20 phosphoproteins), regardless of whether or not they produced statistically significant ANOVA comparisons between groups or locations at $\mathrm{p}<0.05$. 
IPA uses the relative up- and down-regulation of targets compared to a control to construct networks, so all 31 targets were normalized by correcting the concentration/relative phosphorylation values at each location to the contralateral leg for every individual animal. This method allowed for correction arising from biological variability and provided some correction for systemic inflammation. These normalized responses were investigated with QIAGEN's Ingenuity ${ }^{\circledR}$ Pathway Analysis (IPA ${ }^{\circledR}$, QIAGEN, Redwood City), and proposed networks were created for each group (Sham, Implant Only, Inf+Implant) at each of the three locations (Op Prox, Op Knee, Op Dist). The IPA networks consist of nodes from the experimental dataset and literature-derived projected nodes likely to be involved, identified by Ingenuity Knowledge Base. Up- and down-regulated responses are color coded using red and green, respectively. IPA also reported top up- and down-regulated targets for each of the nine networks, based on changes in expression across groups.

\subsection{Results of phosphoprotein data and IPA networks}

\subsubsection{Ten phosphoproteins were elevated in response to implant or infection}

Phosphoprotein levels were compared between groups and locations, and ten of the twenty phosphoproteins tested exhibited increased phosphorylation levels when implant or infection was present: MEK1 (S217/S221), RPS6 (S235/S236), p70S6K (T389), PTEN (S380), BAD (S136), p90RSK (S380), VEGFR2 (Y1175), ERK1/2 (T202/Y204 T185/Y187), IKB- $\alpha$ (S32/S36), and c-

Jun (S63). Trends between the three groups are specifically discussed in the following paragraphs.

MEK1, RPS6, p70S6K, PTEN, BAD, p90RSK, VEGFR2, and IkB- $\alpha$ showed increases in both Implant Only and Inf+Implant groups compared to Sham at a statistically significant level $(\mathrm{p}<0.05)$ at a minimum of one tissue location. ERK1/2 also appeared to follow this trend, but it 
did not produce statistically different phosphorylation levels compared to the Sham group (Fig. 3.5). Additionally, some of these cytokines produced significant comparisons between the Implant Only and Inf+Implant groups at $\mathrm{p}<0.05$ at certain tissue locations. p70S6K and BAD showed differences between Implant Only and Inf+Implant proximal to the operative site $(\mathrm{p}<0.05)$, and p90RSK was significantly higher in Inf+Implant compared to Sham and Implant Only directly at the operative site (Fig. 3.5, p<0.05). c-Jun did not show as much of a disparity in relative phosphorylation levels between the three groups compared to the other targets tested, with the only significant comparison at $\mathrm{p}<0.05$ being Inf+Implant at the operative knee compared to both Sham and Implant Only at this location (Fig. 3.5). 

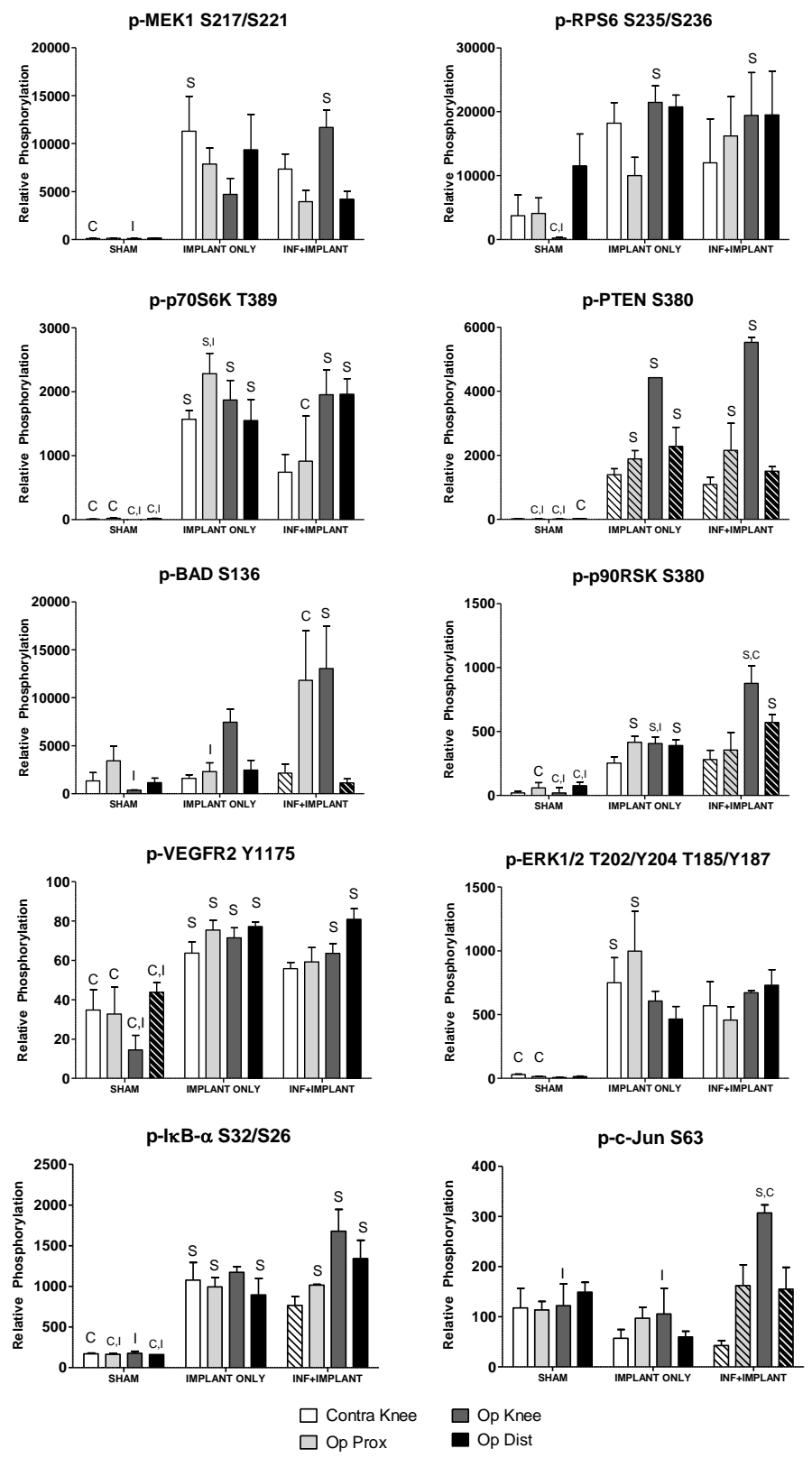

Figure 3. 5. Ten phosphoproteins showed elevated levels in response to implant and infection. Relative phosphorylation levels were compared between all samples at a concentration of $900 \mu \mathrm{g} / \mathrm{mL}$ total protein. Statistically significant differences $(\mathrm{p}<0.05)$ in relative phosphorylation between Sham $(\mathrm{N}=6)$, Implant Only $(\mathrm{N}=6)$, and $\mathrm{Inf}+\operatorname{Implant}(\mathrm{N}=6)$ groups are marked for each location: "S" represents different than Sham group; "C" denotes different than Implant Only group; "I" denotes different than Inf+Implant group. All locations were compared to the operative knee site within each group, and statistically significant differences between locations are marked with striped bars to indicate the concentration at that location is different than the operative knee at $\mathrm{p}<0.05$. 


\subsubsection{Five phosphoproteins showed increased responses proximal or distal to the operative knee}

Of the twenty phosphoproteins tested, five showed trends of increased phosphorylation levels proximal or distal to the operative knee in either the Implant Only group, Inf+Implant group, or both. CREB (S133), Smad2 (S165/S167), and Syk (Y352) showed phosphorylation levels at the operative distal site that were significantly higher than the Implant Only or Sham groups $(\mathrm{p}<0.05)$. For all three of these targets, the operative distal location was not significantly different at $\mathrm{p}<0.05$ from the operative knee site within the Inf+Implant group. In other words, phosphorylation of CREB, Smad2, and Syk in the Inf+Implant group was similar between these two locations and significantly higher $(\mathrm{p}<0.05)$ than the other two groups.

Phosphorylation levels of AKT (S473) and p38 (Y180/Y182) trended upward in both the Implant Only and Inf+Implant groups and were higher than levels in the Sham group (Fig. 3.6). Phosphorylated p38 was significantly higher at the operative distal site than the operative knee in the Inf+Implant group $(\mathrm{p}<0.05)$; the Implant Only group only exhibited an increase, but it was not at a statistically significant level. Additionally, both AKT and p38 were present at significantly higher levels in the operative proximal tissues compared to operative knee in the Implant Only group. However, this was not the case for the Inf+Implant group (Fig. 3.6). 
p-CREB S133

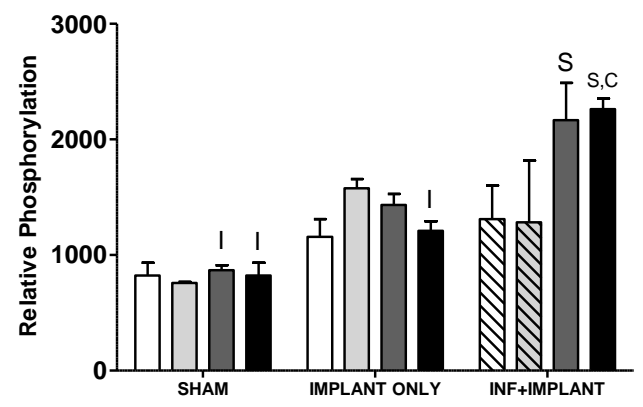

p-Syk Y352

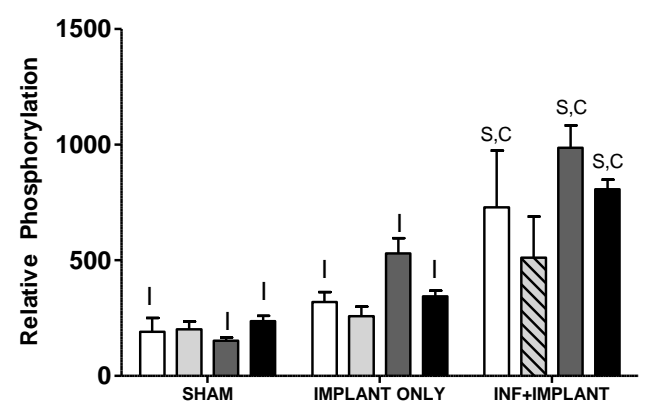

p-p38 Y180/Y182

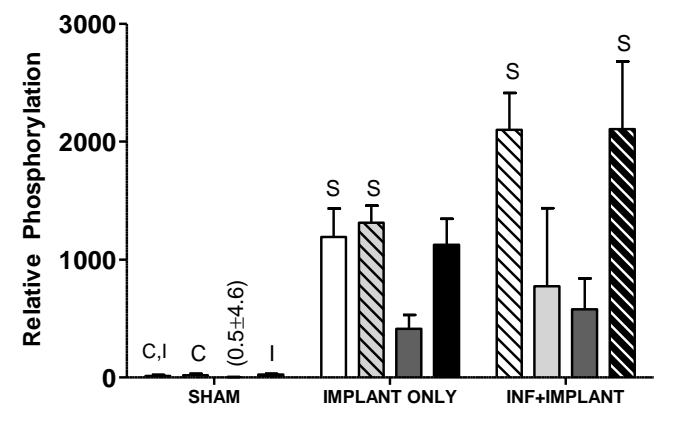

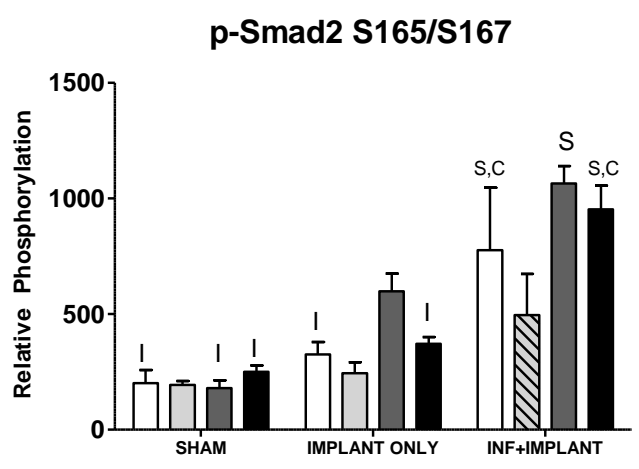

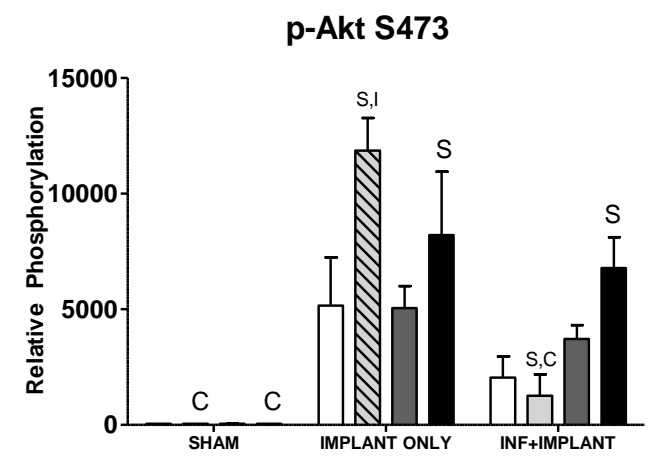

Figure 3. 6. Five phosphoproteins showed elevated levels proximal or distal to the opeartive knee site. Relative phosphorylation levels were compared between all samples at a concentration of $900 \mu \mathrm{g} / \mathrm{mL}$ total protein. Statistically significant differences $(\mathrm{p}<0.05)$ in relative phosphorylation between Sham $(\mathrm{N}=6)$, Implant Only $(\mathrm{N}=6)$, and Inf+Implant $(\mathrm{N}=6)$ groups are marked for each location: "S" represents different than Sham group; "C" denotes different than Implant Only group; "I" denotes different than Inf+Implant group. All locations were compared to the operative knee site within each group, and statistically significant differences between locations are marked with striped bars to indicate the concentration at that location is different than the operative knee at $\mathrm{p}<0.05$. 


\subsubsection{IPA revealed differences in wound healing responses}

IPA was used to construct network of responses for all nine networks (3 groups, 3 tissue locations) from experimentally-derived and literature-derived data (Fig. 3.7). A qualitative analysis of connectivity of the networks was conducted by quantifying the number of edges in each individual network. An "edge" is a connection between two nodes, and IPA draws edges between nodes by quantifying the overlap via a Fisher's Exact Test with significance set to $p<0.01$. A higher number of edges in a network signifies greater connectivity, since there are more relationships between sets of nodes. In this study, the following number of edges were identified in each network (with number of edges shown in parenthesis after the network name): Op Prox Sham (101), Op Knee Sham (99), Op Dist Sham (101), Op Prox Implant Only (98), Op Knee Implant Only (104), Op Dist Implant Only (99), Op Prox Inf+Implant (99), Op Knee Inf+Implant (99), Op Dist Inf+Implant (96). The average number of edges was 99.6, so all networks were within 5 edges of the average.

While the connectivity was similar between all nine networks, construction (i.e., the position of nodes) differed. An analysis of important up- and down-regulated targets was conducted to better understand which nodes may differ between the networks. Changes in expression were compared, with a positive change signifying up-regulation and a negative change denoting downregulation. The top three nodes for up- and down-regulation from the experimental data set were identified at the operative knee joint, and the behavior of these nodes is graphed between all three locations to show a comparison between Op Prox, Op Knee, and Op Dist (Fig. 3.8). 

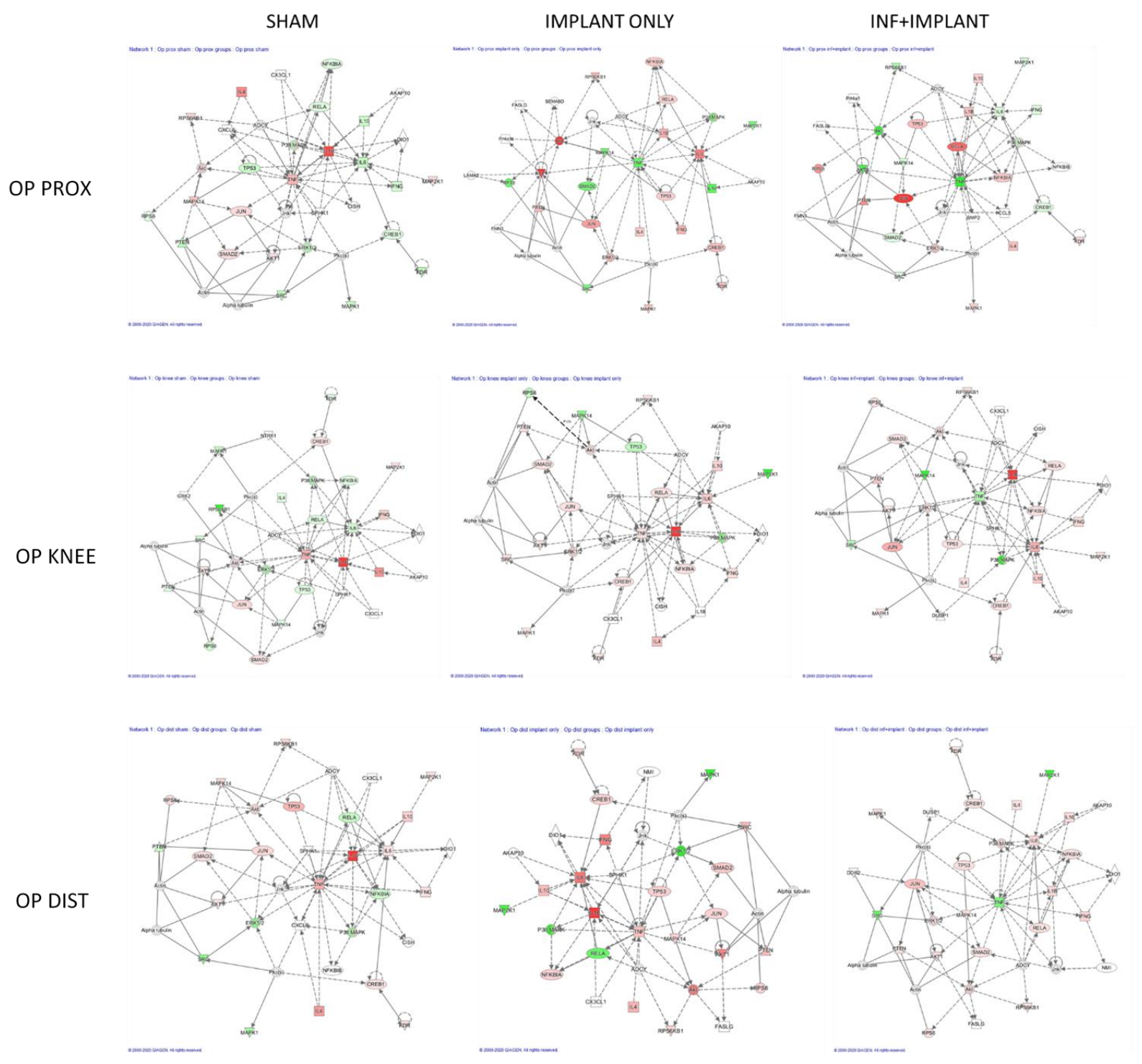

Figure 3. 7. Ingenuity Pathway Analysis (IPA)-generated networks. Proposed networks used relative cytokine and phosphoprotein responses. Nodes are illustrated in a "heat map" coloring scheme, with red denoting up-regulation, green denoting down-regulation, and the intensity of color correlates to the intensity of relative response. The networks are supplemented with other nodes likely to be involved, as identified in the Ingenuity Knowledge Base. A solid line represents a direct interaction between two nodes, while a dotted line denotes an indirect relationship. 

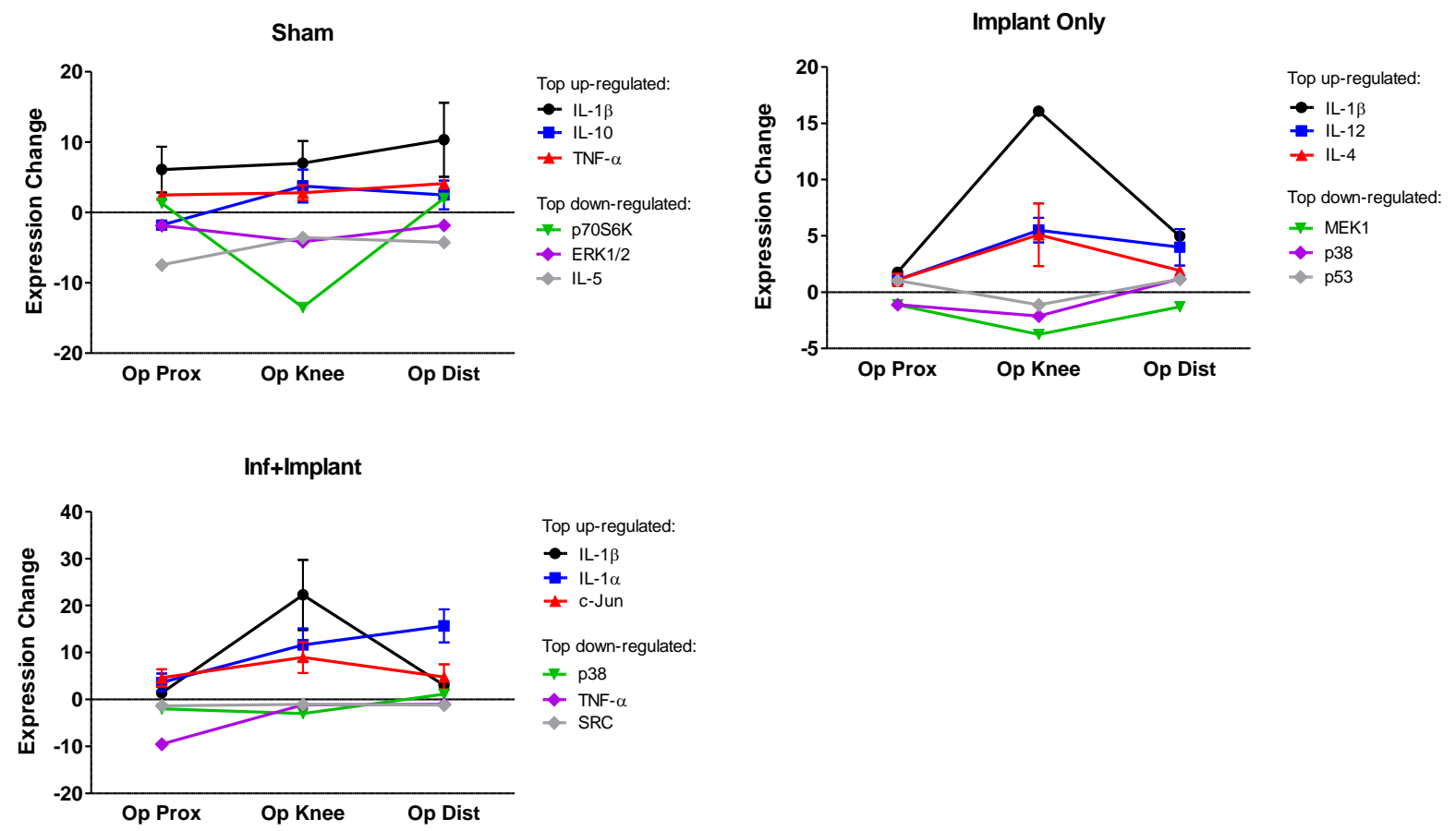

Figure 3. 8. Comparison of top up- and down-regulated nodes compared to the operative knee. Differences in expression for the top three up-regulated and down-regulated nodes from the experimental data set are shown for each of the three groups. The average expression change \pm SEM is shown for each node. 


\subsection{Discussion of Wound Healing Network Analysis}

Network analysis provided some clarity on the aspects of tissues upstream and downstream from the operative knee that may affect immune response at operative knee location. It is well established that the cytokines investigated in this study play important roles in the inflammatory response to implants or infection [2], and to better understand the impacts on cell signaling related to wound healing, phosphoproteins were included in a follow-up analysis. These phosphoproteins are related to wound healing processes such as proliferation [68-70], fibrosis and establishment of connective tissue [71-73], and inflammatory apoptosis [74-76]. Changes in expression levels of these targets between locations may highlight some of the disruptions observed for cytokines at the operative proximal or operative distal sites.

While the overall connectivity of networks of cytokines and phosphoproteins analyzed by IPA was similar based on the number of edges, the construction differed as well as the up- and down-expression of nodes. Top contributors were identified in each of the nine networks by highlighting the top three up-regulated and down-regulated nodes. Changes between tissue locations for these top network contributors may indicate targets that highly influence the response [77]. Within the Sham group, comparison of the top up- and down-regulated targets between locations revealed that phosphoproteins p70S6K and ERK1/2 were higher at proximal and distal locations compared to the operative knee $(\mathrm{p}<0.05)$, and both of these phosphoproteins are important for proliferative processes in wound healing [70]. Cytokine IL-5 expressed higher directly at the joint than the proximal location $(\mathrm{p}<0.05)$ but was not significantly different than the distal location (Fig. 3.8). IL-5 is a mediator of TH2 immunity and promotes eosinophil-mediated inflammation [78], so its elevation at the operative knee and operative distal locations may signify 
that there is a higher degree of inflammation at these locations, which agrees with the cytokine data as a whole (Figs. 3.2 and 3.3). In the Implant Only group, pro-inflammatory IL-1 $\beta$ was significantly lower at the proximal and distal locations compared to the operative knee $(\mathrm{p}<0.05)$, and IL-12p70 showed the same trend at the proximal tissue $(\mathrm{p}<0.05)$, suggesting that a potent proinflammatory response is present at the operative knee in response to implants. Three phosphoproteins, MEK1, p38, and p53 were all significantly higher at both the proximal and distal locations compared to the operative knee $(\mathrm{p}<0.05)$. These phosphoproteins are important for wound healing during multiple stages, including proliferation [69], migration [74], and apoptotic processes in wound healing [71]. Following the same trend at the Sham group, the Implant Only group showed higher cytokine expression at the operative knee and lower levels of wound healing phosphoproteins. In the Inf+Implant group, pro-inflammatory IL-1 $\beta$ was significantly higher at the operative knee than proximal and distal to this site $(\mathrm{p}<0.05)$, and TNF- $\alpha$ was lower in proximal tissues $(\mathrm{p}<0.05)$. Phosphoprotein p38 was expressed in higher levels at the operative distal location compared to the joint site $(\mathrm{p}<0.05)$.

There were several limitations to this study. The cytokine concentrations were measured in rodents that were treated with anesthetics and analgesics. Many studies have observed suppression of cytokine production following administration of these agents $[57,58]$. The use of isoflurane and buprenorphine was specifically chosen to avoid significant modulation of cytokine response [37]. Buprenorphine has been shown to have no significant effect on cytokine production in rodents [59]. While isoflurane has been shown to increase levels of pro-inflammatory cytokines like IL-1 $\beta$, IL-6, and TNF- $\alpha$ [60], comparison with the Sham group still illustrated an increase in levels of these cytokines due to both implants and infection. Many approaches to studying chronic 
joint infections like PJI have been pursued in literature [61], and this study represents only one infecting organism, S. aureus, at one post-surgical time point in a non-load bearing K-wire implant model. Future studies will focus on varying durations of infection, include other infecting organisms, and multiple inoculation doses.

In conclusion, the follow-up network analysis provided some insight into the spatiallydependent tissue response, which may have implications healing. When comparing the operative proximal and operative distal locations to the operative knee, phosphoproteins identified in IPA as top contributors- p70S6K, ERK1/2, MEK1, p38, and p53- were consistently higher at proximal and distal sites (Fig. 3.8). Cytokines IL-5, IL-1b, IL-12p70, and TNF- $\alpha$ were also identified as top contributors in IPA, and these were consistently higher at the operative knee. Further, IL-5, IL12 p70, and TNF- $\alpha$ were significantly lower proximal to the joint in the Sham, Implant Only, and Inf+Implant groups, respectively, suggesting that the distal location may maintain a higher degree of inflammation similar to the operative site. The network analysis allowed identification of likely contributors to differences in healing responses, and these results suggested that cytokines dominate the healing response at the operative knee in all three groups, while wound healingrelated phosphoproteins govern the response at proximal and distal sites.

\subsection{Acknowledgements}

Funding for this work was provided by NIH K Award 1K08AR073921. The authors would like to thank Suzanne Danley for her editorial suggestions. 


\subsection{References}

[1] Tande AJ, Patel R: Prosthetic joint infection. Clin Microbiol Rev 2014, 27:302-345.

[2] Seebach E, Kubatzky KF: Chronic Implant-Related Bone Infections-Can Immune Modulation be a Therapeutic Strategy? Front Immunol 2019, 10:1724.

[3] Ma CY, Lu YD, Bell KL, Wang JW, Ko JY, Wang CJ, Kuo FC: Predictors of Treatment Failure After 2-Stage Reimplantation for Infected Total Knee Arthroplasty: A 2- to 10-Year Follow-Up. J Arthroplasty 2018, 33:2234-2239.

[4] Aali Rezaie A, Goswami K, Shohat N, Tokarski AT, White AE, Parvizi J: Time to Reimplantation: Waiting Longer Confers No Added Benefit. J Arthroplasty 2018, 33:1850-1854.

[5] Kandel CE, Jenkinson R, Daneman N, Backstein D, Hansen BE, Muller MP, Katz KC, Widdifield J, Bogoch E, Ward S, et al: Predictors of Treatment Failure for Hip and Knee Prosthetic Joint Infections in the Setting of 1- and 2-Stage Exchange Arthroplasty: A Multicenter Retrospective Cohort. Open Forum Infect Dis 2019, 6:ofz452.

[6] Ma L, Zhang H, Yin YL, Guo WZ, Ma YQ, Wang YB, Shu C, Dong LQ: Role of interleukin6 to differentiate sepsis from non-infectious systemic inflammatory response syndrome. Cytokine 2016, 88:126-135.

[7] Punyadeera C, Schneider EM, Schaffer D, Hsu HY, Joos TO, Kriebel F, Weiss M, Verhaegh WF: A biomarker panel to discriminate between systemic inflammatory response syndrome and sepsis and sepsis severity. J Emerg Trauma Shock 2010, 3:26-35.

[8] Deirmengian C, Hallab N, Tarabishy A, Della Valle C, Jacobs JJ, Lonner J, Booth RE: Synovial fluid biomarkers for periprosthetic infection. Clin Orthop Relat Res 2010, 468:20172023. 
[9] Shahi A, Kheir MM, Tarabichi M, Hosseinzadeh HRS, Tan TL, Parvizi J: Serum D-Dimer

Test Is Promising for the Diagnosis of Periprosthetic Joint Infection and Timing of Reimplantation. J Bone Joint Surg Am 2017, 99:1419-1427.

[10] Signore A, Sconfienza LM, Borens O, Glaudemans AWJM, Cassar-Pullicino V, Trampuz A, Winkler H, Gheysens O, Vanhoenacker FMHM, Petrosillo N, Jutte PC: Consensus document for the diagnosis of prosthetic joint infections: a joint paper by the EANM, EBJIS, and ESR (with ESCMID endorsement). Eur J Nucl Med Mol Imaging 2019, 46:971-988.

[11] Gomes LSM: Early Diagnosis of Periprosthetic Joint Infection of the Hip-Current Status, Advances, and Perspectives. Rev Bras Ortop (Sao Paulo) 2019, 54:368-376.

[12] Alijanipour P, Bakhshi H, Parvizi J: Diagnosis of periprosthetic joint infection: the threshold for serological markers. Clin Orthop Relat Res 2013, 471:3186-3195.

[13] Li C, Renz N, Trampuz A: Management of Periprosthetic Joint Infection. Hip Pelvis 2018, 30:138-146.

[14] Schulte W, Bernhagen J, Bucala R: Cytokines in sepsis: potent immunoregulators and potential therapeutic targets--an updated view. Mediators Inflamm 2013, 2013:165974.

[15] Dinarello CA: Historical insights into cytokines. Eur J Immunol 2007, 37 Suppl 1:S34-45.

[16] Turner MD, Nedjai B, Hurst T, Pennington DJ: Cytokines and chemokines: At the crossroads of cell signalling and inflammatory disease. Biochim Biophys Acta 2014, 1843:2563-2582.

[17] Nilsdotter-Augustinsson A, Briheim G, Herder A, Ljunghusen O, Wahlström O, Ohman L: Inflammatory response in 85 patients with loosened hip prostheses: a prospective study comparing inflammatory markers in patients with aseptic and septic prosthetic loosening. Acta Orthop 2007, 78:629-639. 
[18] Frangiamore SJ, Siqueira MB, Saleh A, Daly T, Higuera CA, Barsoum WK: Synovial Cytokines and the MSIS Criteria Are Not Useful for Determining Infection Resolution After Periprosthetic Joint Infection Explantation. Clin Orthop Relat Res 2016, 474:1630-1639.

[19] Gollwitzer H, Dombrowski Y, Prodinger PM, Peric M, Summer B, Hapfelmeier A, Saldamli B, Pankow F, von Eisenhart-Rothe R, Imhoff $\mathrm{AB}$, et al: Antimicrobial peptides and proinflammatory cytokines in periprosthetic joint infection. J Bone Joint Surg Am 2013, 95:644651.

[20] Monastero RN, Pentyala S: Cytokines as Biomarkers and Their Respective Clinical Cutoff Levels. Int J Inflam 2017, 2017:4309485.

[21] Sousa R, Abreu MA: Treatment of Prosthetic Joint Infection with Debridement, Antibiotics and Irrigation with Implant Retention - a Narrative Review. J Bone Jt Infect 2018, 3:108-117.

[22] Shahi A, Tan TL, Kheir MM, Tan DD, Parvizi J: Diagnosing Periprosthetic Joint Infection: And the Winner Is? J Arthroplasty 2017, 32:S232-S235.

[23] del Pozo JL, Patel R: The challenge of treating biofilm-associated bacterial infections. Clin Pharmacol Ther 2007, 82:204-209.

[24] Li C, Renz N, Trampuz A, Ojeda-Thies C: Twenty common errors in the diagnosis and treatment of periprosthetic joint infection. Int Orthop 2020, 44:3-14.

[25] Qu X, Zhai Z, Li H, Liu X, Zhu Z, Wang Y, Liu G, Dai K: PCR-based diagnosis of prosthetic joint infection. J Clin Microbiol 2013, 51:2742-2746.

[26] Cannon JG, Tompkins RG, Gelfand JA, Michie HR, Stanford GG, van der Meer JW, Endres S, Lonnemann G, Corsetti J, Chernow B: Circulating interleukin-1 and tumor necrosis factor in septic shock and experimental endotoxin fever. J Infect Dis 1990, 161:79-84. 
[27] Werner S, Grose R: Regulation of wound healing by growth factors and cytokines. Physiol Rev 2003, 83:835-870.

[28] Teijaro JR: Cytokine storms in infectious diseases. Semin Immunopathol 2017, 39:501-503.

[29] Soetens JFJ, Worsley PR, Bader DL, Oomens CWJ: Investigating the influence of intermittent and continuous mechanical loading on skin through non-invasive sampling of IL-1 $\alpha$. J Tissue Viability 2019, 28:1-6.

[30] Currie HN, Loos MS, Vrana JA, Dragan K, Boyd JW: Spatial cytokine distribution following traumatic injury. Cytokine 2014, 66:112-118.

[31] Adams CS, Antoci V, Harrison G, Patal P, Freeman TA, Shapiro IM, Parvizi J, Hickok NJ, Radin S, Ducheyne P: Controlled release of vancomycin from thin sol-gel films on implant surfaces successfully controls osteomyelitis. J Orthop Res 2009, 27:701-709.

[32] Peng KT, Hsieh CC, Huang TY, Chen PC, Shih HN, Lee MS, Chang PJ: Staphylococcus aureus biofilm elicits the expansion, activation and polarization of myeloid-derived suppressor cells in vivo and in vitro. PLoS One 2017, 12:e0183271.

[33] Lucke M, Schmidmaier G, Sadoni S, Wildemann B, Schiller R, Stemberger A, Haas NP, Raschke M: A new model of implant-related osteomyelitis in rats. J Biomed Mater Res B Appl Biomater 2003, 67:593-602.

[34] Penn-Barwell JG, Rand BC, Brown KV, Wenke JC: A versatile model of open-fracture infection : a contaminated segmental rat femur defect. Bone Joint Res 2014, 3:187-192.

[35] Morris JL, Letson HL, Grant A, Wilkinson M, Hazratwala K, McEwen P: Experimental model of peri-prosthetic infection of the knee caused by. Biol Open 2019, 8 . 
[36] Fan Y, Xiao Y, Sabuhi WA, Leape CP, Gil D, Grindy S, Muratoglu OK, Bedair H, Collins JE, Randolph M, Oral E: Longitudinal Model of Periprosthetic Joint Infection in the Rat. J Orthop Res 2020, 38:1101-1112.

[37] Al-Mousawi AM, Kulp GA, Branski LK, Kraft R, Mecott GA, Williams FN, Herndon DN, Jeschke MG: Impact of anesthesia, analgesia, and euthanasia technique on the inflammatory cytokine profile in a rodent model of severe burn injury. Shock 2010, 34:261-268.

[38] Aktekin CN, Ozturk AM, Tabak AY, Altay M, Korkusuz F: A different perspective for radiological evaluation of experimental osteomyelitis. Skeletal Radiol 2007, 36:945-950.

[39] Askar M, Ashraf W, Scammell B, Bayston R: Comparison of different human tissue processing methods for maximization of bacterial recovery. Eur J Clin Microbiol Infect Dis 2019, $38: 149-155$.

[40] Hulse RE, Kunkler PE, Fedynyshyn JP, Kraig RP: Optimization of multiplexed bead-based cytokine immunoassays for rat serum and brain tissue. J Neurosci Methods 2004, 136:87-98.

[41] Kurtz SM, Lau E, Watson H, Schmier JK, Parvizi J: Economic burden of periprosthetic joint infection in the United States. J Arthroplasty 2012, 27:61-65.e61.

[42] Shah K, Mohammed A, Patil S, McFadyen A, Meek RM: Circulating cytokines after hip and knee arthroplasty: a preliminary study. Clin Orthop Relat Res 2009, 467:946-951.

[43] Fröschen FS, Schell S, Schildberg FA, Klausing A, Kohlhof H, Gravius S, Randau TM: Analysis of synovial biomarkers with a multiplex protein microarray in patients with PJI undergoing revision arthroplasty of the hip or knee joint. Arch Orthop Trauma Surg 2020.

[44] Zhang JM, An J: Cytokines, inflammation, and pain. Int Anesthesiol Clin 2007, 45:27-37. 
[45] Li J, Bower AJ, Vainstein V, Gluzman-Poltorak Z, Chaney EJ, Marjanovic M, Basile LA, Boppart SA: Effect of recombinant interleukin-12 on murine skin regeneration and cell dynamics using in vivo multimodal microscopy. Biomed Opt Express 2015, 6:4277-4287.

[46] Sprague AH, Khalil RA: Inflammatory cytokines in vascular dysfunction and vascular disease. Biochem Pharmacol 2009, 78:539-552.

[47] Han AA, Currie HN, Loos MS, Vrana JA, Fabyanic EB, Prediger MS, Boyd JW: Spatiotemporal phosphoprotein distribution and associated cytokine response of a traumatic injury. Cytokine 2016, 79:12-22.

[48] Egea L, Hirata Y, Kagnoff MF: GM-CSF: a role in immune and inflammatory reactions in the intestine. Expert Rev Gastroenterol Hepatol 2010, 4:723-731.

[49] Lee A, Park SP, Park CH, Kang BH, Park SH, Ha SJ, Jung KC: IL-4 Induced Innate CD8+ T Cells Control Persistent Viral Infection. PLoS Pathog 2015, 11:e1005193.

[50] Xie K, Dai K, Qu X, Yan M: Serum and Synovial Fluid Interleukin-6 for the Diagnosis of Periprosthetic Joint Infection. Sci Rep 2017, 7:1496.

[51] Fillerova R, Gallo J, Radvansky M, Kraiczova V, Kudelka M, Kriegova E: Excellent Diagnostic Characteristics for Ultrafast Gene Profiling of. J Clin Microbiol 2017, 55:2686-2697.

[52] Deirmengian C, Kardos K, Kilmartin P, Cameron A, Schiller K, Parvizi J: Diagnosing periprosthetic joint infection: has the era of the biomarker arrived? Clin Orthop Relat Res 2014, 472:3254-3262.

[53] Lopez-Castejon G, Brough D: Understanding the mechanism of IL-1 $\beta$ secretion. Cytokine Growth Factor Rev 2011, 22:189-195.

[54] Tanaka T, Narazaki M, Kishimoto T: IL-6 in inflammation, immunity, and disease. Cold Spring Harb Perspect Biol 2014, 6:a016295. 
[55] Bergsbaken T, Fink SL, Cookson BT: Pyroptosis: host cell death and inflammation. Nat Rev Microbiol 2009, 7:99-109.

[56] Zhang J, Bárdos T, Shao Q, Tschopp J, Mikecz K, Glant TT, Finnegan A: IL-4 potentiates activated T cell apoptosis via an IL-2-dependent mechanism. J Immunol 2003, 170:3495-3503.

[57] Hong JY, Lim KT: Effect of preemptive epidural analgesia on cytokine response and postoperative pain in laparoscopic radical hysterectomy for cervical cancer. Reg Anesth Pain Med 2008, 33:44-51.

[58] Kawasaki T, Ogata M, Kawasaki C, Ogata J, Inoue Y, Shigematsu A: Ketamine suppresses proinflammatory cytokine production in human whole blood in vitro. Anesth Analg 1999, 89:665669.

[59] Peterson NC, Nunamaker EA, Turner PV: To Treat or Not to Treat: The Effects of Pain on Experimental Parameters. Comp Med 2017, 67:469-482.

[60] Wu X, Lu Y, Dong Y, Zhang G, Zhang Y, Xu Z, Culley DJ, Crosby G, Marcantonio ER, Tanzi RE, Xie Z: The inhalation anesthetic isoflurane increases levels of proinflammatory TNF- $\alpha$, IL-6, and IL-1ß. Neurobiol Aging 2012, 33:1364-1378.

[61] Carli AV, Ross FP, Bhimani SJ, Nodzo SR, Bostrom MP: Developing a Clinically Representative Model of Periprosthetic Joint Infection. J Bone Joint Surg Am 2016, 98:1666-1676.

[62] Koh TJ, DiPietro LA: Inflammation and wound healing: the role of the macrophage. Expert Rev Mol Med 2011, 13:e23.

[63] O'Connor SM, Taylor CE, Hughes JM: Emerging infectious determinants of chronic diseases. Emerg Infect Dis 2006, 12:1051-1057.

[64] Szkaradkiewicz A, Karpiński TM, Zeidler A, Szkaradkiewicz AK, Masiuk H, GiedrysKalemba S: Cytokine response in patients with chronic infections caused by Staphylococcus 
aureus strains and diversification of their Agr system classes. Eur J Clin Microbiol Infect Dis 2012, $31: 2809-2815$.

[65] Morel PA, Lee REC, Faeder JR: Demystifying the cytokine network: Mathematical models point the way. Cytokine 2017, 98:115-123.

[66] Thomas S, Bonchev D: A survey of current software for network analysis in molecular biology. Hum Genomics 2010, 4:353-360.

[67] Ghosh S, Zang S, Mitra PS, Ghimbovschi S, Hoffman EP, Dutta SK: Global gene expression and Ingenuity biological functions analysis on PCBs 153 and 138 induced human PBMC in vitro reveals differential mode(s) of action in developing toxicities. Environ Int 2011, 37:838-857.

[68] Couture C, Desjardins P, Zaniolo K, Germain L, Guérin SL: Enhanced wound healing of tissue-engineered human corneas through altered phosphorylation of the CREB and AKT signal transduction pathways. Acta Biomater 2018, 73:312-325.

[69] Vollmar B, El-Gibaly AM, Scheuer C, Strik MW, Bruch HP, Menger MD: Acceleration of cutaneous wound healing by transient p53 inhibition. Lab Invest 2002, 82:1063-1071.

[70] Matsubayashi Y, Ebisuya M, Honjoh S, Nishida E: ERK activation propagates in epithelial cell sheets and regulates their migration during wound healing. Curr Biol 2004, 14:731-735.

[71] Reid MB, Li YP: Cytokines and oxidative signalling in skeletal muscle. Acta Physiol Scand 2001, 171:225-232.

[72] Lin L, White SA, Hu K: Role of p90RSK in Kidney and Other Diseases. Int J Mol Sci 2019, 20.

[73] Bao P, Kodra A, Tomic-Canic M, Golinko MS, Ehrlich HP, Brem H: The role of vascular endothelial growth factor in wound healing. J Surg Res 2009, 153:347-358. 
[74] Yang M, Guan DW, Xiong CY, Cheng ZH, Yu TS: [Expression of c-jun during the incised wound healing in mice skin]. Fa Yi Xue Za Zhi 2009, 25:401-404.

[75] Zhao M: PTEN: a promising pharmacological target to enhance epithelial wound healing. Br J Pharmacol 2007, 152:1141-1144.

[76] Downward J: How BAD phosphorylation is good for survival. Nat Cell Biol 1999, 1:E3335.

[77] Chen SJ, Liao DL, Chen CH, Wang TY, Chen KC: Construction and Analysis of ProteinProtein Interaction Network of Heroin Use Disorder. Sci Rep 2019, 9:4980.

[78] Takatsu K: Interleukin-5 and IL-5 receptor in health and diseases. Proc Jpn Acad Ser B Phys Biol Sci 2011, 87:463-485. 


\section{Chapter 4}

Impact of Cytokines and Phosphoproteins in Response to Chronic Joint Infection 


\section{Impact of Cytokines and Phosphoproteins in Response to Chronic Joint Infection ${ }^{3}$}

The early cellular response to infection has been investigated extensively, generating valuable information regarding the mediators of acute infection response. Various cytokines have been highlighted for their critical roles, and the actions of these cytokines are related to intracellular phosphorylation changes to promote infection resolution. However, the development of chronic infections has not been thoroughly investigated. While it is known that wound healing processes are disrupted, the interactions of cytokines and phosphoproteins that contribute to this dysregulation are not well understood. To investigate these relationships, this study used a network centrality approach to assess the impact of individual cytokines and phosphoproteins during chronic inflammation and infection. Tissues were taken from patients undergoing total knee arthroplasty (TKA) and total knee revision (TKR) procedures across two tissue depths to understand which proteins are contributing most to the dysregulation observed at the joint. Notably, p-c-Jun, p-CREB, p-BAD, IL-10, IL-12p70, IL-13, and IFN- $\gamma$ contributed highly to the network of proteins involved in aseptic inflammation caused by implants. Similarly, p-PTEN, IL-4, IL-10, IL-13, IFN- $\gamma$, and TNF- $\alpha$ appear to be central to signaling disruptions observed in septic joints. Ultimately, the network centrality approach provided insight into the altered tissue responses observed in chronic inflammation and infection.

3 Parts of this chapter have been published previously from Prince N, Penatzer JA, Dietz, MJ, and Boyd, JW. Impact of Cytokines and Phosphoproteins in Response to Chronic Joint Infection. Biology. 9, 167 (2020). 


\subsection{Introduction}

Acute responses to inflammation and infection have been well studied in literature, and these studies have highlighted important roles for many cytokines [1-3] and phosphoproteins [4,5] in early inflammatory immune processes. The coordinated series of signaling events involves the recruitment of pro-inflammatory regulators like IL-1 $\alpha$, IL-1 $\beta$, and IL-6 [6-8] to the site, provoking intracellular phosphorylation changes of many mitogen-activated protein kinase (MAPK/ERK) mediators [9-11]. This acute inflammatory response to infection is predictable. However, less is known about the transition that leads to the development of chronic infections [12]. Chronic, persistent infections are challenging to treat and can present a challenge for clinicians [13]. Periprosthetic joint infection (PJI) is an infection surrounding a prosthetic knee and represents one example of localized infections that can transition into a chronic state. Dysregulation of immune mediators has been observed systemically for PJI $[14,15]$, but the mechanisms that lead to these signaling disruptions have not been investigated [16]. PJI affects approximately 40,000 patients per year in the United States [17], and resolving these chronic infections is a high priority for clinicians. These patients suffer from chronic inflammation surrounding the joint due to presence of implant as well as infection [18,19]. This compound inflammation makes the tissue-level response difficult to understand using traditional statistical approaches. Further investigation into the tissue-level disruptions that lead to chronic infection and inflammation may allow a better understanding of how best to address these conditions.

Network analysis approaches allow for a global evaluation of these complex, tissue-level disruptions [20]. Traditional statistical methods for evaluating these contributions may be limited, as they can only evaluate one component individually. Conversely, network analysis approaches 
allow for an understanding of the interactions of different components with respect to the entire signaling network [20]. Currently, pathway analysis software like Ingenuity Pathway Analysis (IPA), Cytoscape, and iPathway Guide are used to analyze these types of datasets from a network perspective, and these tools offer an enriched understanding of biological networks. These applications allow users to construct networks, analyze molecular functions, and identify disease states using experimental and literature-derived data [21,22].

Beyond literature-based enrichment of data, mathematical modeling, such as network centrality parameter analysis, can be used to dissect large datasets and understand relationships between the individual components. Network centrality parameters assign quantitative values to every measured target (node) to describe how central each target is relative to all other nodes in the network. Some examples of centrality parameters are degree (number of direct neighbors), diameter (maximum distance between nodes in the network), and radiality (shortest path between a node and all other nodes, normalized to network diameter) [23].

A node with a high radiality indicates that node is central to the network, and networks with mostly high radiality nodes are behaving in an organized manner. Conversely, nodes with low centrality values have peripheral roles, and networks with many low radiality nodes may be interpreted as an open cluster of proteins that are connecting to other regulatory molecules [23]. By focusing on the nodes with low centrality outcomes, it may be possible to understand which peripheral nodes are contributing to the dysregulation observed in networks of chronic inflammation and infection that occur in TKR patients, especially those suffering from PJI. 
Radiality has been used in literature to probe biological networks and garner information about protein-protein interactions to understand chronic inflammatory conditions like diabetes [24], cancer [25], and chronic viral infections [26]. Ultimately, using radiality to evaluate these signaling networks allowed an opportunity to identify new therapeutic targets to combat these conditions. Evaluating the nodes that are most central and most peripheral in chronic infections like PJI may yield similar benefits.

In this study, nine cytokines and twenty-one phosphoproteins were measured in tissues surrounding the knee joint to evaluate differences between native response in primary TKA, chronic inflammatory response in aseptic TKR, and chronic infection response in septic TKR. Two tissue depths were evaluated for each group: adjacent tissue layer (ATL), unhealthy tissue that is close to the joint and requires removal; and radial tissue layer (RTL), healthy tissue that does not need to be removed. The dataset was examined using IPA and network centrality radiality to allow both qualitative and quantitative evaluations of cytokine and phosphoprotein contributions. A comparison of radiality values between primary TKA, aseptic TKR, and septic TKR allowed for a narrowing of the nodes with particularly distinct responses. These nodes may have important contributions to the disruption of normal cell signaling events. In the future, a focused analysis of these protein targets may facilitate the development of new therapeutics to combat persistent inflammation and infection observed in these patients. 


\subsection{Materials and methods}

\subsubsection{Patient cohort}

All subjects gave informed consent for inclusion in the study, and the study was conducted in accordance with the Declaration of Helsinki. Following Institutional Review Board (IRB) approval (IRB protocol \#1709745853) and patient consent, six patients undergoing primary total knee arthroplasty (TKA) and eleven patients undergoing total knee revision (TKR) procedures participated in the study (8 males and 9 females; aged 45-82 years; body max index [BMI] 24.643.7; information can be found in Table 4.1), and subjects were recruited over a 12-month period. All six primary TKA patients were undergoing elective surgery for total replacement of the knee joint with a diagnosis of osteoarthritis. In the TKR group, patients were further characterized into aseptic and septic revision procedures. Patients with aseptic revisions $(\mathrm{N}=5)$ were undergoing revisions due to failures of the prosthetic joint but did not show presence of infection. Patients with septic revisions $(\mathrm{N}=6)$ met clinical criteria for a PJI diagnosis, as defined by the Musculoskeletal Infection Society (MSIS) criteria [27]. All six patients diagnosed with PJI had positive tissue cultures on the day of surgery: four tested culture positive for Staphylococcus epidermidis, one for Methicillin-sensitive Staphylococcus aureus (MSSA), and one for Enterobacter cloacae. All groups of patients received the same pre-operative pain relief and anesthesia, per standard clinical procedures. 
Table 4. 1. Patient Information. Six primary TKA and eleven revision TKR patients were enrolled in the study, creating a heterogenous cohort of males and females varying in age (45-82 years) and comorbidities. Primary TKA patients have ID format P\#; revision TKR patients have ID format F\#. This table lists general patient information including the pathogen for which each septic patient tested culture-positive following testing on the day of surgery. Serum CRP values were obtained pre-operatively in the revision setting. Cultures were obtained from intraoperative tissue samples.

\begin{tabular}{|c|c|c|c|c|c|c|}
\hline ID & Sex & TKA/TKR & BMI $\left(\mathrm{kg} / \mathrm{m}^{2}\right)$ & Diabetic (Y/N) & CRP (mg/L) & Culture \\
\hline $\mathrm{P} 1$ & $\mathrm{~F}$ & TKA & 33.8 & $\mathrm{~N}$ & N/A & Negative \\
\hline $\mathrm{P} 2$ & $\mathrm{~F}$ & TKA & 39.8 & $\mathrm{~N}$ & N/A & Negative \\
\hline P3 & $\mathrm{F}$ & TKA & 39.8 & $\mathrm{~N}$ & N/A & Negative \\
\hline $\mathrm{P} 4$ & $\mathrm{M}$ & TKA & 29.7 & $\mathrm{Y}$ & N/A & Negative \\
\hline P5 & $\mathrm{M}$ & TKA & 24.6 & $\mathrm{~N}$ & N/A & Negative \\
\hline P6 & $\mathrm{M}$ & TKA & 27.2 & $\mathrm{~N}$ & N/A & Negative \\
\hline F1 & $\mathrm{F}$ & TKR-Aseptic & 28.2 & $\mathrm{~N}$ & 4.3 & Negative \\
\hline $\mathrm{F} 2$ & $\mathrm{~F}$ & TKR- Aseptic & 29.8 & $\mathrm{~N}$ & 0.2 & Negative \\
\hline F3 & $\mathrm{F}$ & TKR-Aseptic & 33.9 & $\mathrm{~N}$ & $<1$ & Negative \\
\hline F4 & $\mathrm{M}$ & TKR-Aseptic & 40.4 & $\mathrm{Y}$ & 3.6 & Negative \\
\hline F5 & $\mathrm{M}$ & TKR- Aseptic & 26.2 & $\mathrm{~N}$ & 2.1 & Negative \\
\hline F6 & $\mathrm{F}$ & TKR-Septic & 43.7 & $\mathrm{~N}$ & 28.8 & S. epidermidis \\
\hline F7 & $\mathrm{F}$ & TKR-Septic & 30.8 & $\mathrm{Y}$ & 161.4 & S. epidermidis \\
\hline F8 & $\mathrm{F}$ & TKR-Septic & 41.9 & $\mathrm{~N}$ & 21.7 & E. cloaecae \\
\hline F9 & $\mathrm{M}$ & TKR-Septic & 36.2 & $\mathrm{~N}$ & 33.5 & MSSA \\
\hline F10 & $\mathrm{M}$ & TKR-Septic & 33.8 & $\mathrm{Y}$ & 3.8 & S. epidermidis \\
\hline F11 & $M$ & TKR-Septic & 31.9 & $\mathrm{~N}$ & 111.9 & S. epidermidis \\
\hline
\end{tabular}




\subsubsection{Collection of tissue samples}

All TKA and TKR procedures were performed by a single surgeon with standard debridement and washing protocols. Debridement during TKA and TKR is the removal of unhealthy tissue surrounding the joint [28]. Tissues were collected at a total of four distinct anatomical locations, shown in Figure 4.1. The solid line circle represents location 1: medial femoral condyle (F); the dashed line circle represents location 2: medial tibial plateau (T); the solid line square represents location 3: lateral gutter (LG); and the dashed line square represents location 4: posterior capsule (PC). These tissues were collected at two tissue layers, the adjacent tissue layer (ATL) and radial tissue layer (RTL). The ATL samples came from the initial debridement; these tissues are removed during surgery to promote better wound healing. RTL samples were taken from a tissue layer further removed from the joint after the surgeon completed debridement. The difference in depth of the RTL tissues and ATL tissues was $\sim 1 \mathrm{~cm}$. Anatomical locations $1-4$ were collected for the ATL layer, and locations 1-3 were collected for the RTL layer. Location 4 (PC) could not be taken in the RTL layer due to proximity to neurovascular structures. Therefore, a total of seven tissue samples were taken for each patient. 


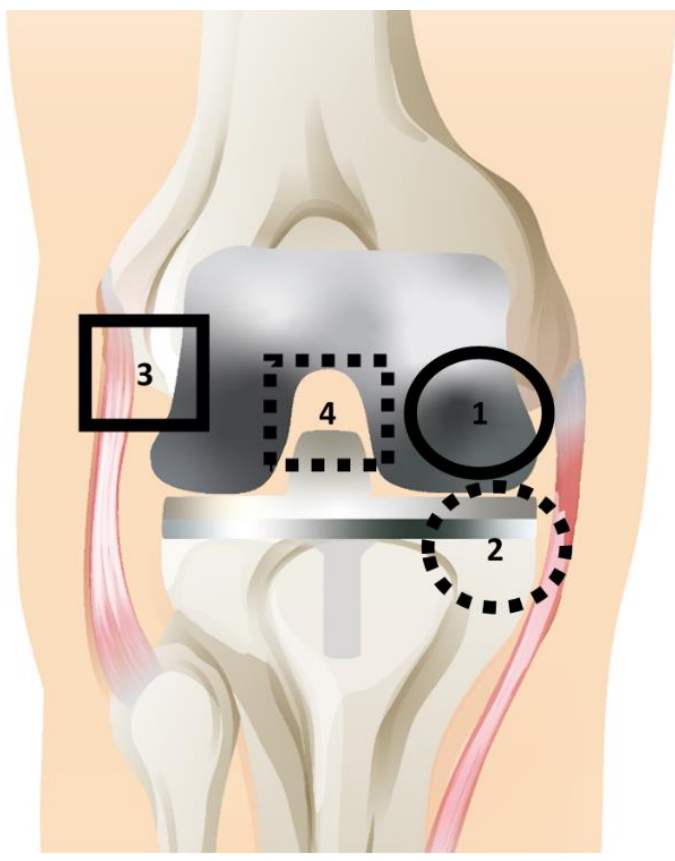

Figure 4. 1. Map of approximate tissue collection locations, shown with prosthetic implant. Seven tissue samples were taken for each patient; (1) the solid circle represents the medial femoral condyle (denoted as F); (2) the dashed circle represents the medial tibial plateau (denoted as T); (3) the solid square represents the lateral gutter (denoted as LG); (4) the dashed square represents the posterior capsule (denoted as PC). Locations 1-4 were taken for the ATL layer, and locations 1-3 were taken for the RTL layer; separation between ATL (unhealthy tissue, closer to joint) and RTL (healthy tissue, further from joint) was approximately $1 \mathrm{~cm}$, depending on individual patient.

\subsubsection{Sample preparation}

Tissues were collected during TKA and TKR procedures in the operating room and immediately stored on dry ice. Once all tissues had been collected for an individual patient, they were washed with $1 \mathrm{X}$ cold phosphate-buffered saline (PBS) to remove blood and debris. Tissues were grossly dissected using a scalpel to remove scar tissue, then stored at $-80{ }^{\circ} \mathrm{C}$. When samples had been collected for all patients, tissues were thawed on ice and cut into sections approximately $30 \mathrm{mg}$ in size; tissues were homogenized by sonication in $500 \mu \mathrm{L}$ cell lysis solution (Bio-Rad, Hercules, CA) containing 20 mM phenylmethylsulfonyl fluoride (Sigma-Aldrich, St. Louis, MO). 
Protein extraction was performed using methods adapted from Hulse et al. [29]. Thawed samples were vortexed for $1-3 \mathrm{~s}$ and centrifuged at $5000 \times g$ for $5 \mathrm{~min}$ at $4{ }^{\circ} \mathrm{C}$. The supernatant was collected and tested for total protein content using a Pierce BCA Protein Assay Kit (Thermo Scientific, Waltham, MA), according to the manufacturer's instructions. Absorbance values for total protein content were determined on an Infinite M1000 multimode plate reader (Tecan, Raleigh, NC).

\subsubsection{Cytokine and phosphoprotein measurement}

To standardize samples for total protein content, tissue homogenates were individually diluted to a total protein concentration of $900 \mu \mathrm{g} / \mathrm{mL}$ with cell lysis buffer (Bio-Rad). Cytokine and phosphoprotein measurements were performed using magnetic bead-based multiplex Inflammation Human ProcartaPlex panel assays (Invitrogen, Carlsbad, CA) and custom Bio-Plex human phosphoprotein multiplex kits. Targets were measured using a Bio-Plex 200 suspension array system and Pro II Wash Station (Bio-Rad), according to the manufacturer's instructions. All cytokines and phosphoproteins measured in the study are listed in Tables 4.2 and 4.3 along with references for their roles in tissue healing. 
Table 4. 2. Cytokine Targets Measured in Tissue Samples. All cytokines measured in this study are listed along with relevant functions during wound healing. Citations are noted in brackets.

\begin{tabular}{ll}
\hline Cytokine & Relevant Functions in Acute Wound Healing Response \\
\hline IL-1 $\beta$ & Early initiator of infection-driven inflammation [2] \\
\hline IL-4 & Anti-inflammatory cytokine that activates Stat6, suppressing cell death [42] \\
\hline IL-6 & Initiator of early inflammatory response to implants and infection [2] \\
\hline IL-1 $\alpha$ & Early recruitment of immune cells in response to infection [2] \\
\hline IL-10 & $\begin{array}{l}\text { Down-regulator of several inflammatory cytokines (i.e., IL-1, IL-6, IL-12, IFN- } \gamma, \\
\text { TNF- } \alpha \text { ) [43] }\end{array}$ \\
\hline IL- & $\begin{array}{l}\text { Pro-inflammatory cytokine involved in adaptive immunity, produced by activated } \\
\text { immune cells [43] }\end{array}$ \\
\hline ILp70 & Th2-associated cytokine critical in tissue remodeling [44] \\
\hline IFN- $\gamma$ & $\begin{array}{l}\text { Anti-inflammatory cytokine that has been associated with inhibition of wound } \\
\text { healing [43] }\end{array}$ \\
\hline TNF- $\alpha$ & Early pro-inflammatory mediator of inflammation [2] \\
\hline
\end{tabular}


Table 4. 3. Phosphoprotein Targets Measured in Tissue Samples. All phosphoproteins measured in this study are listed as well as the site of phosphorylation and roles in wound healing response. Citations are noted in brackets.

\begin{tabular}{|c|c|}
\hline Phosphoprotein (site) & Relevant Functions in Acute Wound Healing Response \\
\hline p-CREB (Ser133) & $\begin{array}{l}\text { Inhibition of CREB via phosphorylation promotes wound closure } \\
\text { [30] }\end{array}$ \\
\hline p-HSP27 (Ser78) & Activation of HSP27 may inhibit stress-induced apoptosis [31] \\
\hline $\mathrm{p}-\mathrm{I} \kappa \mathrm{B} \alpha(\operatorname{Ser} 32 / \mathrm{Ser} 36)$ & Pro-wound healing, inhibits actions of NF- $\mathrm{kB}$ [32] \\
\hline $\begin{array}{l}\text { p-MEK1 } \\
(\text { Ser217/Ser221) }\end{array}$ & Essential for migration of epithelial layers [33] \\
\hline $\begin{array}{l}\text { p-S6RP } \\
\text { (Ser235/Ser236) }\end{array}$ & Activated during proliferative growth phase [30] \\
\hline $\begin{array}{l}\text { p-Smad2 } \\
(\text { Ser465/Ser467) }\end{array}$ & Regulates keratinocyte migration during proliferation [34] \\
\hline p-Src (Tyr416) & Prome \\
\hline p-Syk & ing [35] \\
\hline p-c-Jun & nune cells in skin wound healing [33] \\
\hline p-AKT (S & Phosphorylation of AKT promotes wound closure [30] \\
\hline p-p53 (Ser15) & $\begin{array}{l}\text { Activated p53 accelerates cutaneous wound healing by increasing } \\
\text { cell proliferation [36] }\end{array}$ \\
\hline p-p38 ( & Activated p38 involved in muscle catabolism [32] \\
\hline $\mathrm{p}-\mathrm{p} 70$ & tion [37] \\
\hline p-PTEI & Pro-c \\
\hline p-ZAP-7 & $\lg [35]$ \\
\hline p-BAD ( & ic fun \\
\hline $\begin{array}{l}\text { p-ERK1/2 } \\
\text { (Thr202/Tyr204) }\end{array}$ & Important for early proliferative response in wound healing [37] \\
\hline $\begin{array}{l}\text { p-GSK-3 } \alpha / \beta \\
\text { (Ser21/Ser9) }\end{array}$ & Controls wound healing and fibrosis progression [30] \\
\hline p-p90RSK (Ser380) & $\begin{array}{l}\text { Downstream effector of MEK/ERK pathway in wound healing, } \\
\text { regulator of cell migration [40] }\end{array}$ \\
\hline & epithelialization [41] \\
\hline p-NF-кB p65 (Ser536) & ked to muscle atrophy and catabolism [32] \\
\hline
\end{tabular}




\subsubsection{Data processing and statistical analysis}

Data were analyzed using Prism 5 (GraphPad, San Diego, CA) and SAS JMP (Cary, NC). Cytokine standard curves were generated using either a four- (4PL) or five-parameter logistic (5PL) regression model, depending on the individual protein. Cytokine concentrations are expressed as picograms of cytokine per milliliter of tissue homogenate $(\mathrm{pg} / \mathrm{mL})$. For purposes of network analyses, these values were normalized to the highest value for each cytokine. For phosphoproteins, relative phosphoprotein levels were measured via multiplex enzyme-linked immunosorbent assay (ELISA), and compared to negative control. These values were normalized to the highest value for each phosphoprotein. Contributions of cytokines and phosphoproteins were analyzed for the ATL and RTL layers. All four tissues from the ATL layer were averaged together to represent ATL depth. The three tissues from the RTL layer were averaged together to represent RTL depth. Samples with fluorescence intensity values below the lower limit of quantitation (LLOQ) or above the upper limit of quantitation (ULOQ) were omitted from statistical comparisons of cytokines and phosphoproteins. Outliers were identified using the $1.5 \mathrm{X}$ interquartile range (IQR) rule and omitted from analysis [45]; these were removed on a case-by-case basis to exclude errant values that may have resulted due to assay variability. Two-way analysis of variance (ANOVA) with Bonferroni's post-test was used to determine significant differences between primary TKA, aseptic TKR, and septic TKR tissue samples at each tissue depth, ATL and RTL. Data are expressed as the mean \pm standard error of the mean $(\mathrm{SEM})$. To examine any potential confounding factors in this cohort, Pearson correlations were analyzed between age, sex, and BMI and all 30 measured targets. A Bonferroni's correction was applied, as described in [46], and the correlations were

analyzed for statistical significance at $p<0.05$. Although there are established correlations in literature between inflammatory mediators and age, sex, and BMI, there were no statistically 
significant correlations observed for this study, which indicates that these parameters were not confounding factors (data not shown).

\subsubsection{Network evaluation with Ingenuity Pathway Analysis (IPA)}

The normalized responses of each target were investigated with QIAGEN’s Ingenuity ${ }^{\circledR}$ Pathway Analysis (IPA ${ }^{\circledR}$, QIAGEN, Redwood City). Proposed signaling networks of cytokines and phosphoproteins were created for all groups (primary TKA, aseptic TKR, septic TKR) at the ATL depth. All networks consist of nodes from the experimental dataset and literature-derived projected nodes likely to be involved, identified by Ingenuity Knowledge Base. Up- and downregulated responses are color coded using red and green, respectively. Briefly, IPA constructs networks building on "Focus Genes" or nodes that are highly interconnected [47]. Values from the experimental dataset influence which nodes are designated as "Focus Genes" and may alter the structure of the networks. IPA also reported top molecular and cellular functions related to the network, with corresponding scores (negative $\log _{10}[p$-value of Fisher's exact test]). The Fisher's exact test ( $p$-value) gives the likelihood of finding the identified Focus Genes by random chance in the Global Molecular Network used by IPA.

\subsubsection{Network centrality parameter analysis}

Euclidean distances between pairs of normalized observations (cytokines and phosphoproteins) were determined for each group (primary, aseptic, septic) and depth (ATL and RTL). The definition of Euclidean distance is given in Equation 4.1: 


$$
E(v, \omega)=\sqrt{\sum_{i=1}^{n}\left(\left(\text { normalized response }_{v_{i}}\right)-\left(\text { normalized response }_{\omega_{i}}\right)\right)^{2}}
$$

where $v$ and $\omega$ represent the 2 responses for which the distance between is being calculated, and $\mathrm{n}$ signifies the replicate number. To construct networks of the relative responses of each group, Euclidean distances for each pair of nodes were used to calculate the node centrality parameter, radiality. Radiality is defined as:

$$
C_{\operatorname{rad}(v)}=\frac{\sum_{\omega \epsilon N}(\Delta G+1-\operatorname{dist}(v, \omega))}{n-1}
$$

where $\Delta \mathrm{G}$ represents the network $(\mathrm{N})$ diameter (maximal path length of the network), dist $(v, \omega)$ is the shortest path between a pair of nodes $v$ and $\omega$, and $n$ is the number of nodes in the network (Equation 4.2). To allow for comparisons between networks, radiality values were normalized to the average radiality for all nodes in the network. Significant radiality values were identified using a threshold value of the average radiality \pm the standard deviation.

\subsection{Results}

\subsubsection{Relative spatial cytokine responses}

Nine cytokines were measured in this study: IL-1 $\alpha$, IL-1 $\beta$, IL-4, IL-6, IL-10, IL-12p70, IL13, IFN- $\gamma$, and TNF- $\alpha$. The responses of these cytokines are shown in Figure 4.2. Cytokine levels were normalized across groups (primary TKA, aseptic TKR, septic TKR) and debridement depths (ATL, RTL) to the highest value for each cytokine. Normalizing by this method is important to 
appropriately weight cytokines equally for network analysis rather than relying on raw concentrations. This weighting is performed to understand the contributions of each node to the network, relative to other nodes. Group-dependent differences were observed, as were spatial differences between debridement depths. Briefly, the aseptic TKR and septic TKR groups had higher cytokine responses than the primary TKA group for all cytokines. IL-1 $\alpha$, IL-1 $\beta$, IL-4, and IL-6 had higher levels in septic TKR than aseptic TKR at a statistically significant level $(p<0.05)$. IL-10 was the only cytokine with a lower relative response in the septic TKR when compared to aseptic TKR at a statistically significant level $(p<0.05)$ IL-12p70 seemed to show the same trend, but was not significant at $p<0.05$. There were also differences between ATL and RTL in septic TKR tissues. For IL-1 $\alpha$, IL-1 $\beta$, and IL-4, there were statistically significant differences between ATL and RTL depths for the septic group $(p<0.05)$. 

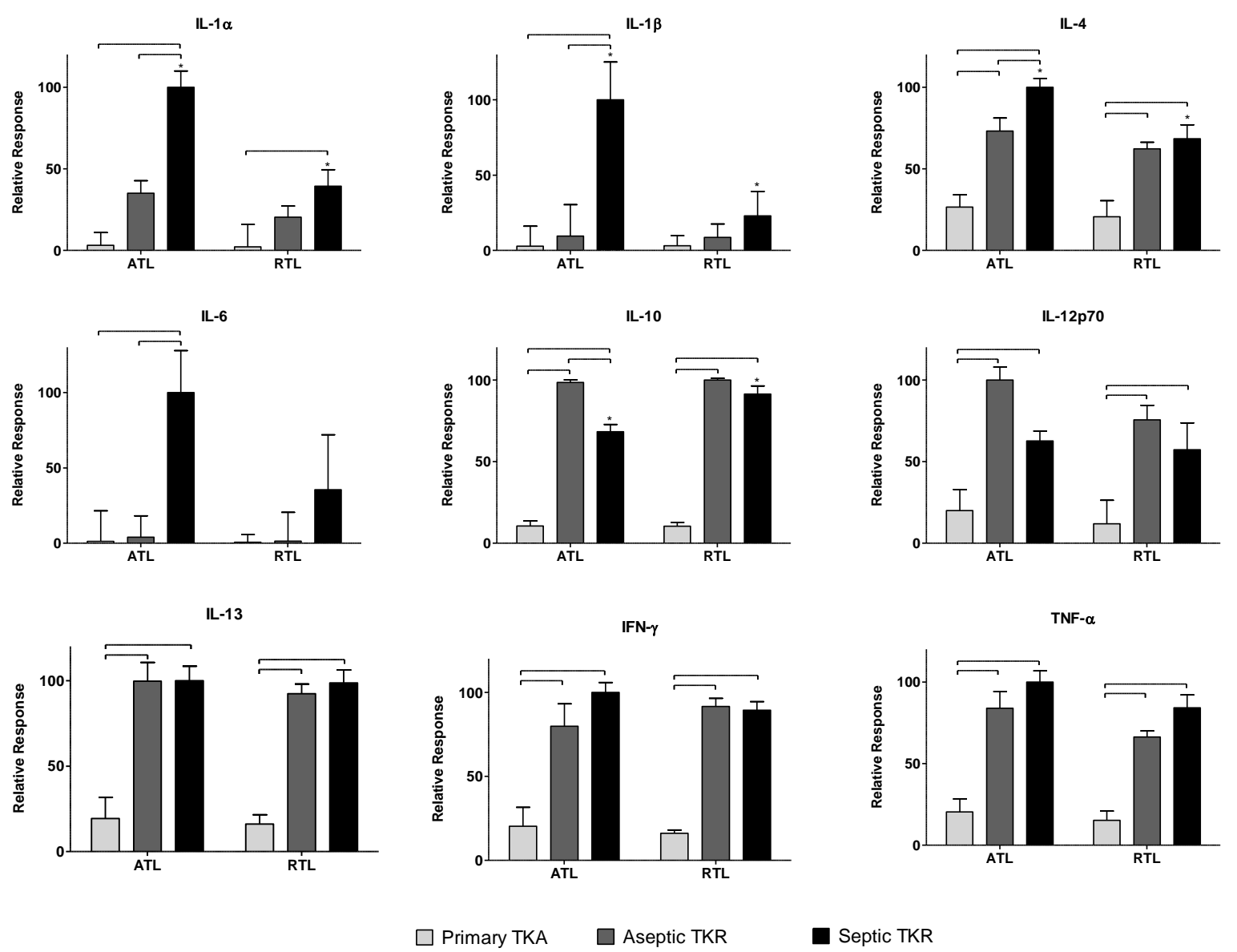

Figure 4. 2. Relative cytokine levels measured in tissues from primary TKA, aseptic TKR, and septic TKR at adjacent tissue layer (ATL) and radial tissue layer (RTL) debridement depths. Relative cytokine responses (normalized to highest cytokine signal) were observed for all three patient groups: primary, aseptic, and septic at two debridement depths: ATL is closer to the knee joint, and RTL is approximately $1 \mathrm{~cm}$ removed from the knee joint. Statistically significant differences $(\mathrm{p}<0.05)$ were determined by two-way ANOVA with Bonferroni's post-test to examine group-dependent and spatially-dependent differences in cytokine relative response. Differences for the same group (i.e., septic) between ATL and RTL are marked with an asterisk $(*)$. Differences between groups within a tissue layer are denoted with bars. Responses are shown as the mean \pm SEM. 


\subsubsection{Relative spatial phosphoprotein responses}

To further investigate the impact of the observed cytokines on tissue response, twenty-one phosphoproteins were measured: p-CREB, p-HSP27, p-IкB- $\alpha$, p-MEK1, p-S6RP, p-Smad2, p-Src, p-Syk, p-c-Jun, p-AKT, p-p53, p-p38, p-p70S6K, p-PTEN, p-ZAP-70, p-BAD, p-ERK1/2, pGSK-3 $\alpha / \beta$, p-p90RSK, pVEGFR2, and p-NF- $\mathrm{BB}$ (more information can be found in Table 4.3). The data are spread over Figures 4.3, 4.4 and 4.5. Figure 4.3 includes phosphoproteins most associated with proliferative wound healing processes [30,36,37,39]. Phosphoproteins in Figure 4.4 have roles in cell migration and fibrotic processes [2,30,32,34,35,40,41]. Finally, Figure 4.5 includes the phosphoproteins that have pro-apoptotic roles and have been associated with delayed wound healing through their involvement in muscle catabolism [33,38-40]. Most phosphoproteins exhibited higher responses in the primary TKA tissues than in aseptic TKR and septic TKR tissues, for both ATL and RTL depths, and many exhibited group-dependent differences, especially in ATL depth. Some exceptions to this trend were p-c-Jun and p-BAD, which had the highest responses in aseptic TKR, then septic TKR, followed by primary TKA; also, p-PTEN showed the highest response in septic tissues (Figure 4.5). Specific group-dependent comparisons are shown in Figures 4.3-4.5.

Tissue depths were also compared for phosphoproteins. Responses in the ATL were higher than responses in the RTL for most phosphoproteins. However, several proteins showed notably higher levels in RTL than ATL for at least one of the three tissue groups: p-BAD, p-Src, p-IкB- $\alpha$, p-HSP27, p-ERK1/2, and p-VEGFR2 (Figures 4.3-4.5). Comparisons of ATL vs. RTL for each group are shown in Figures 4.3-4.5. 

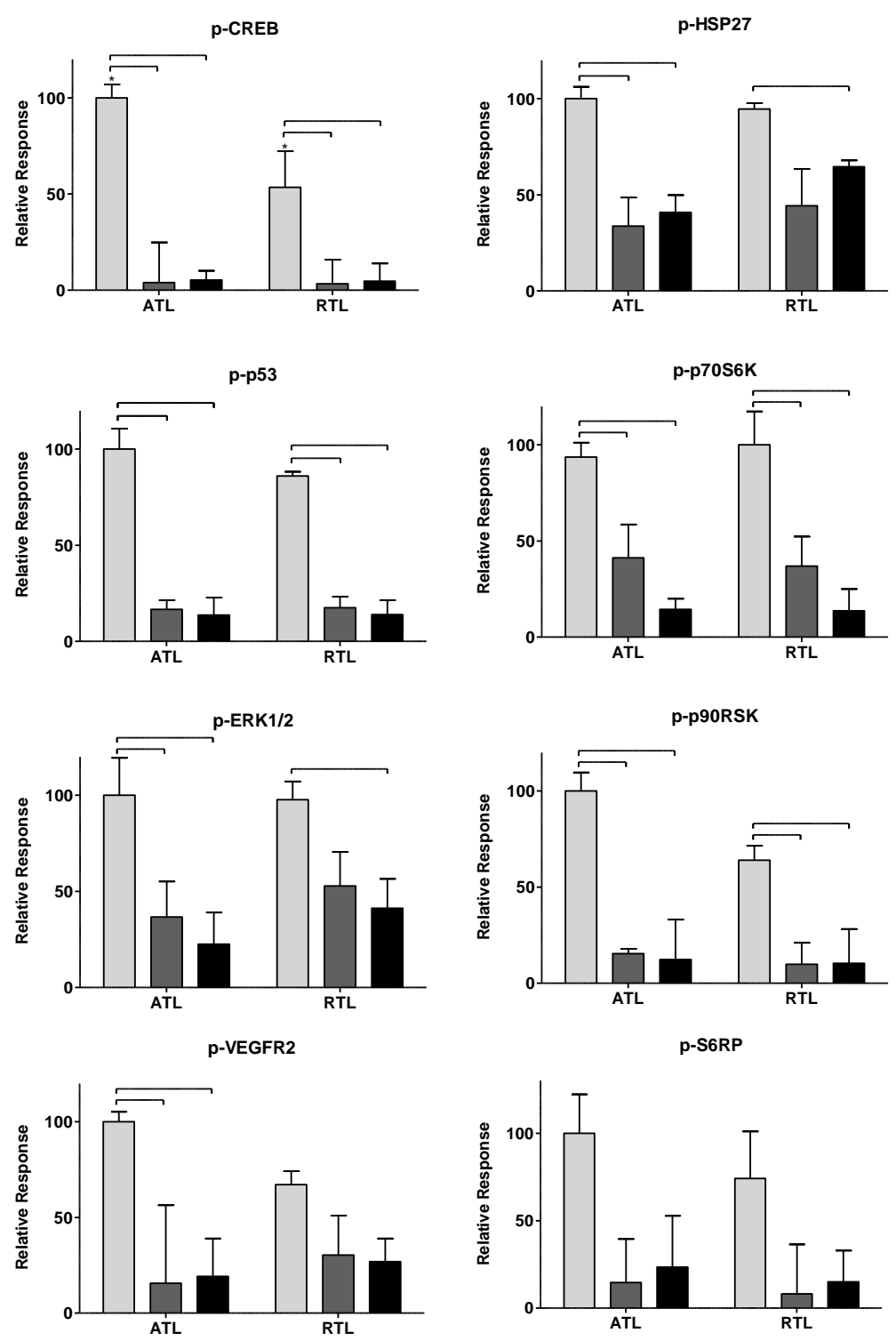

Figure 4. 3. Relative levels of phosphoproteins associated with the proliferative processes in acute wound healing. Relative phosphoprotein responses (normalized to highest signal) were observed for all three patient groups: primary, aseptic, and septic at two debridement depths: ATL is closer to the knee joint, and RTL is approximately $1 \mathrm{~cm}$ removed from the knee joint. Statistically significant differences $(\mathrm{p}<0.05)$ were determined by two-way ANOVA with Bonferroni's posttest to examine group-dependent and spatially-dependent differences in protein phosphorylation. Differences for the same group (i.e., septic) between ATL and RTL are marked with an asterisk $(*)$. Differences between groups within a tissue layer are denoted with bars. Responses are shown as the mean \pm SEM. 

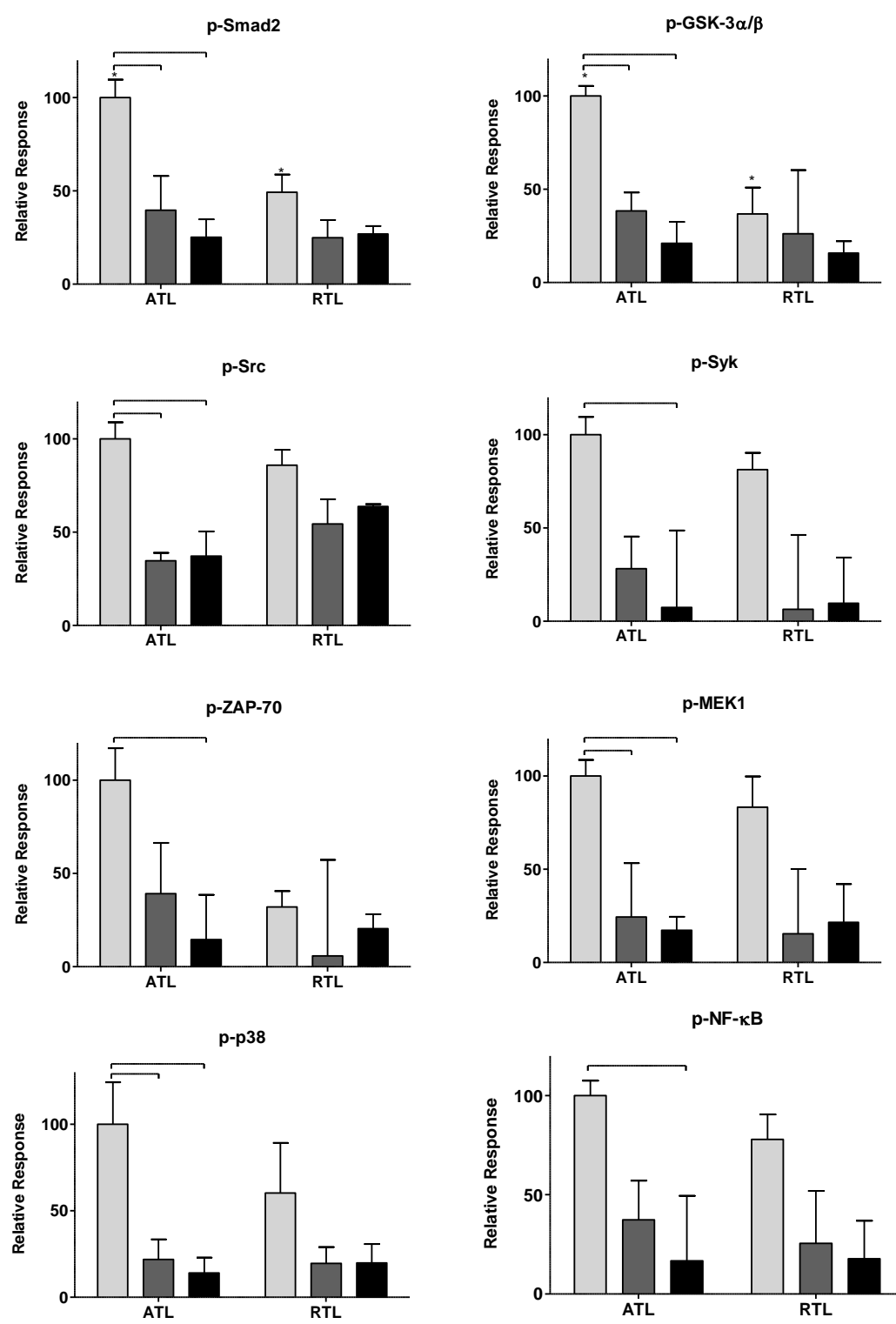

$\square$ Primary TKA

$\square$ Aseptic TKR

Septic TKR

Figure 4. 4. Relative phosphoprotein levels associated with cell migration processes in acute wound healing. Relative phosphoprotein responses (normalized to highest signal) were observed for all three patient groups: primary, aseptic, and septic at two debridement depths: ATL is closer to the knee joint, and RTL is approximately $1 \mathrm{~cm}$ removed from the knee joint. Statistically significant differences $(p<0.05)$ were determined by two-way ANOVA with Bonferroni's posttest to examine group-dependent and spatially-dependent differences in protein phosphorylation. Differences for the same group (i.e., septic) between ATL and RTL are marked with an asterisk $(*)$. Differences between groups within a tissue layer are denoted with bars. Responses are shown as the mean \pm SEM. 

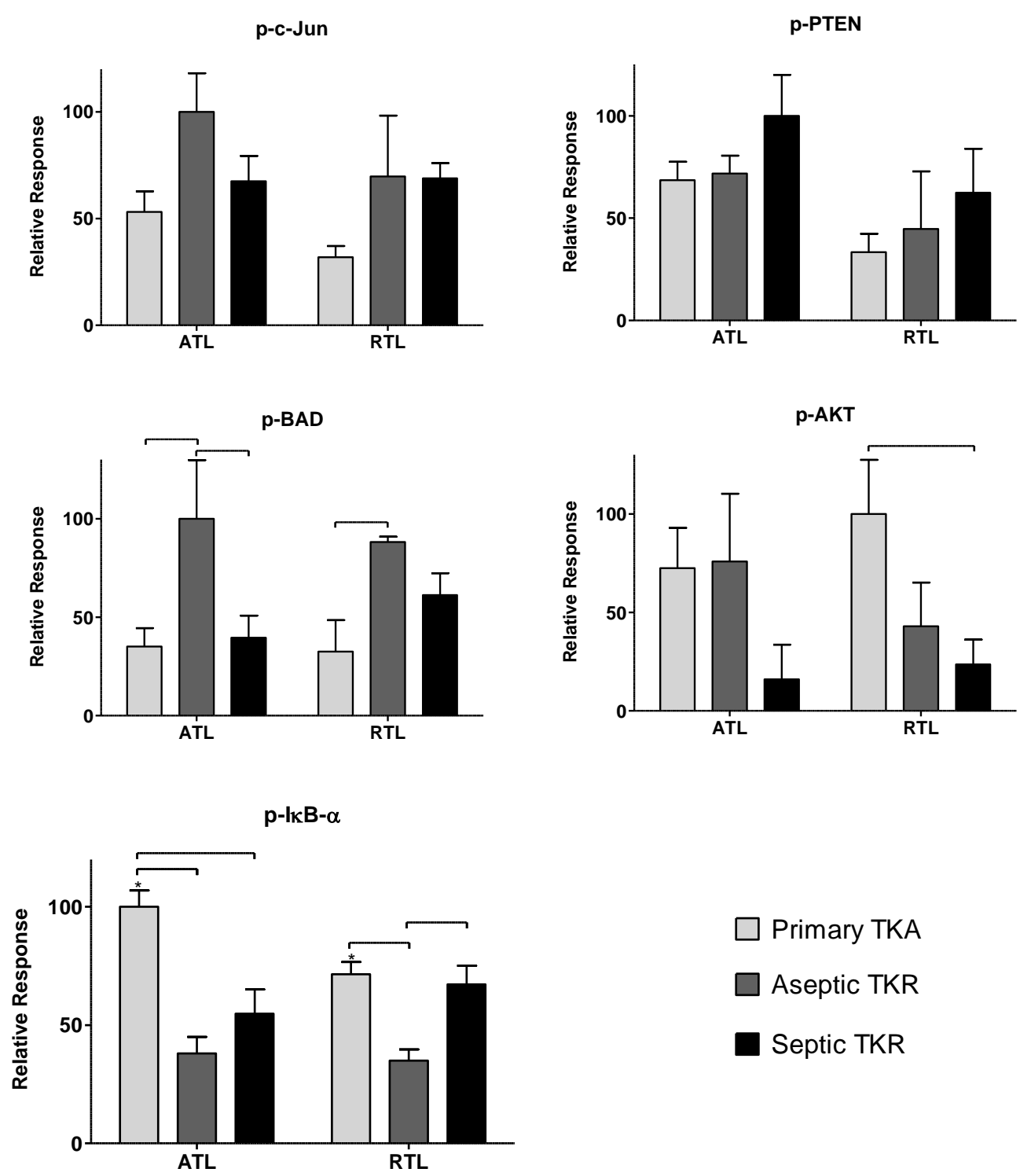

Figure 4. 5. Relative levels of pro-apoptotic and inhibitory wound healing phosphoproteins in acute wound healing. Relative phosphoprotein responses (normalized to highest signal) were observed for all three patient groups: primary, aseptic, and septic at two debridement depths: ATL is closer to the knee joint, and RTL is approximately $1 \mathrm{~cm}$ removed from the knee joint. Statistically significant differences $(p<0.05)$ were determined by two-way ANOVA with Bonferroni's post-test to examine group-dependent and spatially-dependent differences in protein phosphorylation. Differences for the same group (i.e., septic) between ATL and RTL are marked with an asterisk (*). Differences between groups within a tissue layer are denoted with bars. Responses are shown as the mean \pm SEM. 


\subsubsection{IPA-generated networks}

Networks for the three groups (primary TKA, aseptic TKR, and septic TKR) were constructed from the same set of cytokines and phosphoproteins for the ATL layer. The network connectivity varied greatly between the three groups (Figure 4.6). Qualitatively, the primary TKA network showed higher connectivity and more experimentally validated up- and down-regulation of targets, as shown by the red and green coloring, respectively. Further, the connections between targets, also known as "edges," varied between the three groups. Edges denote connections between nodes; in IPA, direct relationships are shown by solid lines, and indirect relationships are shown by dotted lines. The primary TKA network showed 139 edges; 23 of these edges were direct, and 116 were indirect. For aseptic TKR, 65 total edges were identified: 4 direct, 61 indirect. For septic TKR, 61 total edges are shown: 4 direct and 57 indirect. IPA uses the experimental dataset to identify related IPA networks, shown in Table 4.4. A p-score is shown for each IPA network match, and the p-score is calculated based on the $-\log _{10}(p$-value) for the Fisher's exact test. A higher IPA p-score indicates a stronger match; p scores above 21 are generally considered good matches [48]. 


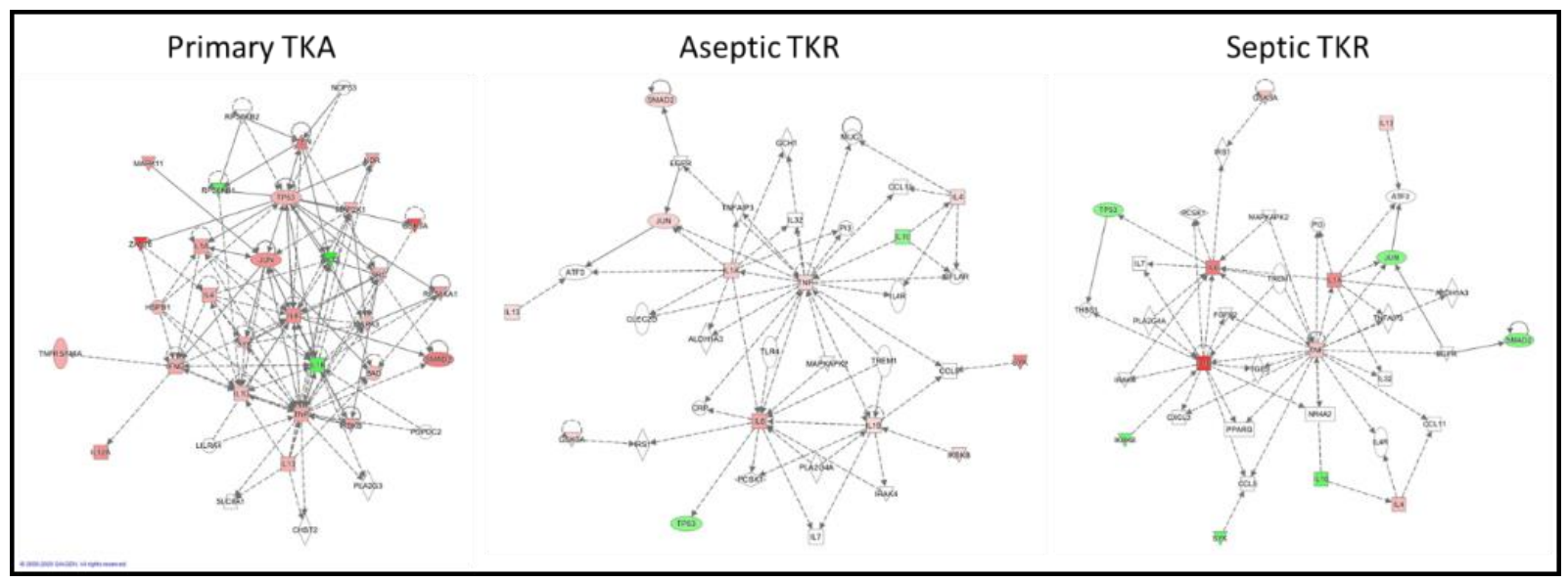

Figure 4. 6. Ingenuity Pathway Analysis (IPA)-generated networks for primary TKA, aseptic TKR, and septic TKR groups based on cytokine and phosphoprotein datasets. Proposed networks used relative cytokine and phosphoprotein responses in the ATL depth, illustrating the differences in tissue responses for the three groups. The nodes are illustrated in a "heat map" coloring scheme, with red denoting up-regulation, green denoting down-regulation, and the intensity of color correlates to the intensity of relative response. The networks are supplemented with other nodes likely to be involved, as identified in the Ingenuity Knowledge Base. A solid line represents a direct interaction between two nodes, while a dotted line denotes an indirect relationship.

Table 4. 4. Top 2 IPA Networks for Primary TKA, Aseptic TKR, and Septic TKR Groups. Network p-scores are calculated by IPA using the negative $\log _{10}$ (p-value) of Fisher's exact test. The p-value describes the probability of finding the cytokines/phosphoproteins randomly in the databases utilized by IPA to construct the network. Networks with p-scores above the threshold of 21 are bolded.

\begin{tabular}{|c|c|c|c|c|c|}
\hline \multicolumn{2}{|l|}{ Primary TKA } & \multicolumn{2}{|l|}{ Aseptic TKR } & \multicolumn{2}{|l|}{ Septic TKR } \\
\hline IPA Network & $\begin{array}{c}\text { p- } \\
\text { score }\end{array}$ & IPA Network & $\begin{array}{c}\text { p- } \\
\text { score }\end{array}$ & IPA Network & $\begin{array}{c}\text { p- } \\
\text { score }\end{array}$ \\
\hline $\begin{array}{c}\text { Cell-mediated immune } \\
\text { response, cellular } \\
\text { development, cellular } \\
\text { function and } \\
\text { maintenance }\end{array}$ & 72 & $\begin{array}{l}\text { Inflammatory response, } \\
\text { cellular movement, cell } \\
\text { death and survival }\end{array}$ & 16 & $\begin{array}{l}\text { Cellular movement, } \\
\text { inflammatory response, } \\
\text { hematological } \\
\text { development and } \\
\text { function }\end{array}$ & 16 \\
\hline $\begin{array}{c}\text { Cancer, organismal injury } \\
\text { and abnormalities, cell } \\
\text { cycle }\end{array}$ & 2 & $\begin{array}{l}\text { Cell-mediated immune } \\
\text { response, cellular } \\
\text { development, cellular } \\
\text { function and } \\
\text { maintenance }\end{array}$ & 9 & $\begin{array}{c}\text { Cell death and survival, } \\
\text { organismal injury and } \\
\text { abnormalities, cellular } \\
\text { development }\end{array}$ & 9 \\
\hline
\end{tabular}




\subsubsection{Normalized radiality of all 30 nodes}

Based on the ANOVA data and IPA-generated networks, all of these cytokine and phosphoprotein targets have roles to play in both infection response and wound healing. To further understand the most important targets, network centrality parameter analysis was performed by analyzing a network centrality parameter, radiality. Radiality values were determined for each cytokine and phosphoprotein node and normalized to the average radiality for the network (e.g., primary TKA, ATL layer). These values are presented in Tables 4.5 and 4.6. Changes in significant radiality outcomes can allow for a better understanding of the "drivers" of each network and deviations from normal response (Figure 4.7). Nodes with significant radiality values are bolded; the significance threshold used was the average radiality \pm standard deviation. Based on previous work [49], we expect significant radiality outcomes with low radiality values to be the most likely drivers of the dysregulation for persistent inflammation and infection of aseptic and septic TKR, respectively. 
Table 4. 5. Normalized Radiality of Nodes in the ATL Layer. Significant target values for each individual network are bolded (significance threshold: the average radiality \pm standard deviation).

\begin{tabular}{|c|c|c|c|}
\hline Node & ATL Primary TKA & ATL Aseptic TKR & ATL Septic TKR \\
\hline p-CREB & 0.96 & 0.77 & 1.15 \\
\hline p-HSP27 & 1.13 & 1.14 & 1.15 \\
\hline p-IкB $\alpha$ & 1.13 & 1.16 & 1.10 \\
\hline p-MEK1 & 1.13 & 1.08 & 1.10 \\
\hline p-S6RP & 1.13 & 0.98 & 1.13 \\
\hline p-Smad2 & 1.13 & 1.15 & 1.14 \\
\hline p-Src & 1.13 & 1.15 & 1.15 \\
\hline p-Syk & 1.13 & 1.11 & 0.95 \\
\hline p-c-Jun & 1.04 & 0.77 & 1.03 \\
\hline p-AKT & 1.10 & 0.99 & 1.08 \\
\hline p-p53 & 1.13 & 1.00 & 1.06 \\
\hline p-p38 & 1.13 & 1.05 & 1.06 \\
\hline p-p70SK6 & 1.13 & 1.15 & 1.07 \\
\hline p-PTEN & 1.09 & 1.02 & 0.76 \\
\hline p-ZAP-70 & 1.13 & 1.16 & 1.07 \\
\hline p-BAD & 0.96 & 0.77 & 1.15 \\
\hline $\mathrm{p}$-ERK1/2 & 1.13 & 1.15 & 1.13 \\
\hline p-GSK-3a/b & 1.13 & 1.16 & 1.12 \\
\hline p-p90RSK & 1.13 & 0.99 & 1.04 \\
\hline $\mathrm{p}$-VEGFR2 & 1.13 & 0.99 & 1.11 \\
\hline p-NF-kB & 1.13 & 1.16 & 1.09 \\
\hline IL-1b & 0.62 & 0.91 & 0.76 \\
\hline IL-4 & 0.90 & 1.01 & 0.76 \\
\hline IL-6 & 0.60 & 0.82 & 0.76 \\
\hline IL-1a & 0.63 & 1.15 & 0.76 \\
\hline IL-10 & 0.73 & 0.79 & 1.02 \\
\hline IL-12p70 & 0.84 & 0.77 & 1.06 \\
\hline IL-13 & 0.84 & 0.77 & 0.76 \\
\hline IFN-y & 0.85 & 0.96 & 0.76 \\
\hline TNF-a & 0.85 & 0.93 & 0.76 \\
\hline
\end{tabular}


Table 4. 6. Normalized Radiality of Nodes in the RTL Layer. Significant target values for each individual network are bolded (significance threshold: the average radiality \pm standard deviation).

\begin{tabular}{|c|c|c|c|}
\hline Node & RTL Primary TKA & RTL Aseptic TKR & RTL Septic TKR \\
\hline p-CREB & 1.12 & 0.79 & 1.06 \\
\hline p-HSP27 & 0.87 & 1.12 & 1.03 \\
\hline p-IкB $\alpha$ & 1.07 & 1.13 & 1.01 \\
\hline p-MEK1 & 0.99 & 1.05 & 1.08 \\
\hline p-S6RP & 1.06 & 0.98 & 1.02 \\
\hline p-Smad2 & 1.12 & 1.11 & 1.10 \\
\hline p-Src & 0.97 & 1.08 & 1.04 \\
\hline p-Syk & 1.01 & 0.96 & 0.94 \\
\hline p-c-Jun & 1.11 & 0.97 & 0.99 \\
\hline p-AKT & 0.80 & 1.12 & 1.09 \\
\hline p-p53 & 0.97 & 1.07 & 1.00 \\
\hline p-p38 & 1.11 & 1.08 & 1.06 \\
\hline p-p70SK6 & 0.80 & 1.13 & 1.00 \\
\hline p-PTEN & 1.12 & 1.12 & 1.05 \\
\hline p-ZAP-70 & 1.11 & 0.96 & 1.07 \\
\hline p-BAD & 1.12 & 0.79 & 1.06 \\
\hline $\mathrm{p}$-ERK1/2 & 0.83 & 1.09 & 1.11 \\
\hline p-GSK-3a/b & 1.12 & 1.12 & 1.02 \\
\hline p-p90RSK & 1.10 & 1.00 & 0.95 \\
\hline p-VEGFR2 & 1.09 & 1.13 & 1.10 \\
\hline p-NF-kB & 1.04 & 1.12 & 1.04 \\
\hline IL-1b & 0.85 & 0.99 & 1.08 \\
\hline IL-4 & 1.04 & 1.03 & 1.00 \\
\hline IL-6 & 0.82 & 0.89 & 1.11 \\
\hline IL-1a & 0.84 & 1.09 & 1.11 \\
\hline IL-10 & 0.95 & 0.64 & 0.70 \\
\hline IL-12p70 & 0.96 & 0.92 & 1.07 \\
\hline IL-13 & 1.01 & 0.74 & 0.59 \\
\hline IFN-y & 1.01 & 0.75 & 0.73 \\
\hline TNF-a & 1.00 & 1.00 & 0.80 \\
\hline
\end{tabular}


While several nodes were significant within each of the six networks, respectively, some nodes showed a group-dependent trend in significance (Tables 4.5 and 4.6). There were changes in significance between the native primary TKA response and aseptic or septic TKR responses. In the primary TKA networks, IL-1 $\alpha$, IL-1 $\beta$, IL-6, and IL-10 gave significant low radiality outcomes for the ATL; p-HSP27, p-AKT, p-ERK1/2, IL-1 $\alpha$, IL-1 $\beta$, and IL-6 were significant in the RTL. Differences for the aseptic TKR group include p-CREB, p-c-Jun, p-BAD, IL12p70, and IL-13 in the ATL; p-CREB, p-BAD, IL-10, IL-13, and IFN- $\gamma$ for the RTL. Deviations in the septic TKR group include p-PTEN, IL-4, IL-13, IFN- $\gamma$, and TNF- $\alpha$ in the ATL layer and IL-10, IL-13, IFN- $\gamma$, and TNF- $\alpha$ in the RTL layer. 

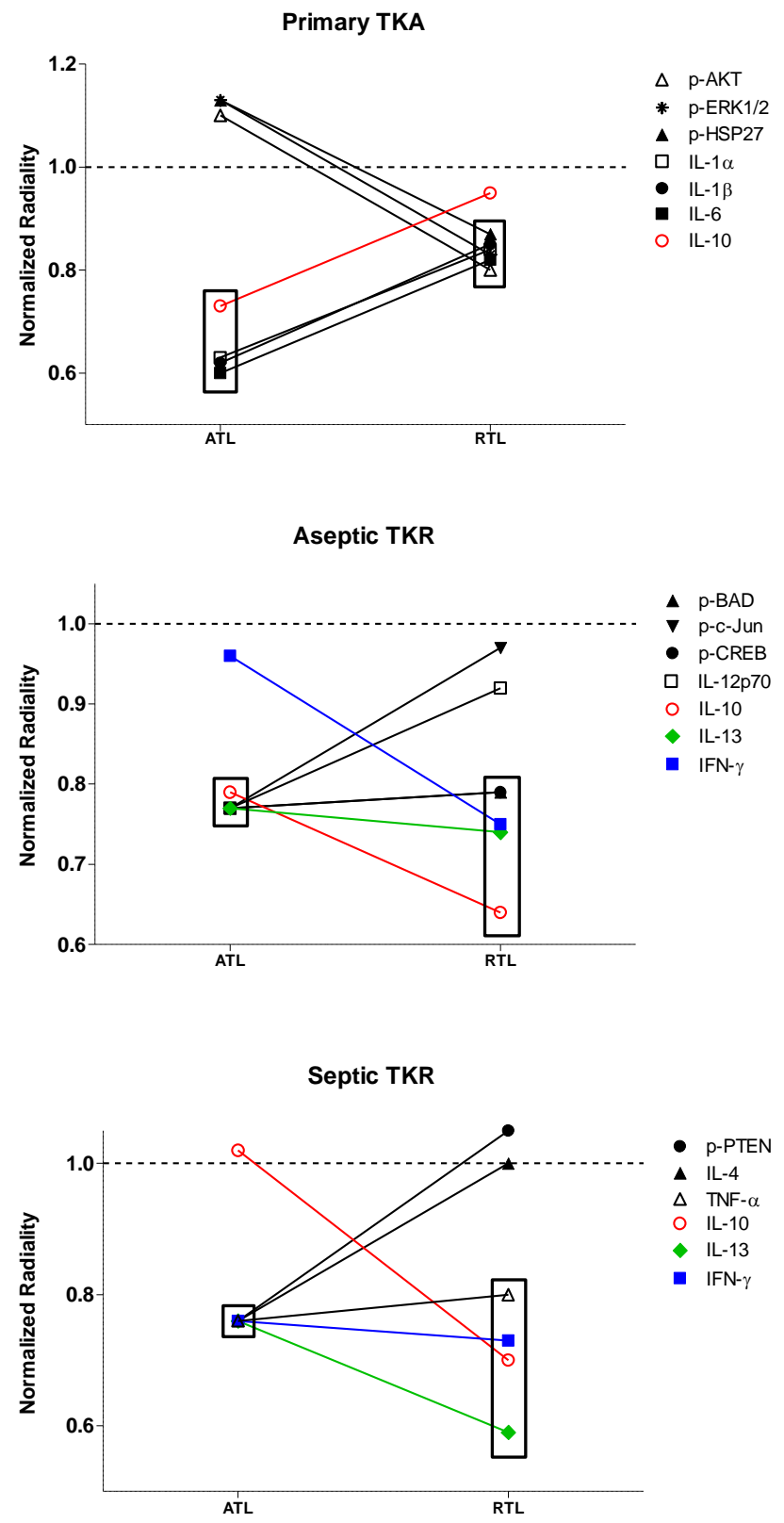

Figure 4. 7. Changes in significant nodes between groups for low radiality outcomes. Nodes with low radiality outcomes that differed between primary TKA response and aseptic/septic TKR responses are shown (significance threshold: the average radiality \pm standard deviation). Boxes indicate significance at varying depths. IL-10 is shown in red to highlight its presence in all three groups: primary TKA, aseptic TKR, and septic TKR. IL-13 (green) and IFN- $\gamma$ (blue) are also colored to highlight overlap in both aseptic TKR and septic TKR groups. 


\subsection{Discussion}

The cytokine and phosphoprotein targets measured in this study are known to be significant contributors to inflammatory responses in general [2], but the interconnected relationships of these targets remain to be elucidated for PJI. Further, many of these targets have not been studied on a tissue level for chronic inflammation and infection, so much of the dysregulation that occurs in immune response and wound healing processes remains unknown [12]. Relative cytokine and phosphoprotein responses were measured to understand the trends in response across three groups of patients: primary TKA, aseptic TKR, and septic TKR at two tissue depths: ATL and RTL. Higher relative cytokine levels were observed in either aseptic or septic TKR samples compared to primary TKA tissues. IL-1 $\alpha$, IL-1 $\beta$, IL-4, and IL-6 showed infection-specific relative responses, with higher levels in septic TKR than both aseptic TKR and primary TKA $(p<0.05$, Figure 4.2). These cytokines have been identified in literature as important early immune response mediators in PJI [50]. Additionally, there were spatial differences between ATL and RTL layers for IL-1 $\alpha$, IL-1 $\beta$, IL-4, and IL-10 (Figure 4.2). The spatial discrepancies observed in this study suggested that the cytokine response is more robust in the ATL layer of septic tissues compared to the RTL. The spatial relationships were unclear for primary TKA and aseptic TKR using ANOVA comparisons (Figure 4.2).

Phosphoproteins were also included in this analysis as many hold central roles in early infection response [4]. The phosphoproteome has not been thoroughly investigated for chronic joint inflammation and infection in PJI, but the relationships between cytokines and phosphoproteins may reveal important information considering the central role of these signaling proteins in cell cycle regulation [9], cell proliferation [36], inflammatory processes [49], and 
wound healing [30]. Most phosphoproteins were found in higher levels in primary TKA tissues (Figures 4.3-4.5). While the septic TKR gave the highest response of most cytokines, it often showed the lowest levels of phosphoproteins (Figures 4.3-4.5). While many of the phosphoproteins tested are downstream targets of cytokines [2,32,33,38,40-43], decreased levels of wound healing-associated phosphoproteins have previously been observed in other studies $[14,15]$. Notable exceptions were p-c-Jun and p-BAD, which were highest in aseptic TKR, and pPTEN, which was highest in septic TKR (Figure 4.5). All three of these phosphoproteins have associated pro-apoptotic functions in acute wound healing [33,38,39], which may be related to their increased phosphorylation in aseptic and septic TKR tissues, respectively. Phosphoprotein levels also showed spatial trends between ATL and RTL at a statistically significant level $(p<$ 0.05) for $\mathrm{p}$-IкB- $\alpha, \mathrm{p}-\mathrm{GSK}-3 \alpha / \beta$, p-Smad2, and p-CREB (Figures 4.3,4.4). All four of these phosphoproteins are related to cell migration and proliferation, and have important roles for wound healing $[30,32,34]$. The results of this study showed higher levels for these phosphoproteins in the ATL of primary TKA, compared to RTL of primary TKA, which suggests tissues closer to the joint have increased wound healing activity (Figures 4.3,4.4).

While traditional ANOVA comparisons gave information about the relative responses of cytokines and phosphoproteins, chronic inflammation and infection involve a series of deeply interconnected targets $[3,12]$, which makes it difficult to fully understand the tissue responses when only considering each target in isolation. The ANOVA data alone do not fully explain which targets may be contributing most to the disruptions in responses observed in aseptic and septic TKR. IPA analysis was used to comparatively assess the connectivity between the three groups. IPA has proven to be a useful tool for visualizing the connectivity of different nodes (i.e., genes, 
proteins, etc.) involved in networks [53]. Figure 4.6 illustrates the utility of IPA for comparing different networks qualitatively and depicts the differences between primary TKA, aseptic TKR, and septic TKR networks for each of the ATL layers. The primary TKA shows better connectivity between targets than aseptic TKR and septic TKR, suggesting there may be dysregulation occurring in both aseptic and septic TKR tissues (Figure 4.6). Additionally, Table 4.4 lists the top IPA network hits for each of the three networks. For proteomic analysis, a p-score above 21 is considered a good match [48]. Only the primary TKA network was able to make a match above this threshold. Based on the IPA analysis, both aseptic TKR and septic TKR networks show a lack of connectivity compared to primary TKA, which may prevent a reliable IPA network match (Table 4.4).

A network centrality approach was also utilized to quantitatively assess which targets were close to (high radiality) or distant from (low radiality) the center of each of the networks. Radiality comparisons may reveal the most likely nodes contributing to the dysregulation observed in the IPA networks. Based on previous work in a rodent model of trauma [49], we expect that differences in nodes with low radiality between primary TKA response and aseptic or septic TKR responses may indicate the most likely causes of disruptions to normal signaling. In this study, low radiality outcomes were the most likely contributors to cell signaling dysregulation leading to chronic inflammation and infection. A significance threshold of the average radiality \pm standard deviation was used to denote significant cytokine and phosphoprotein nodes (Tables 4.5 and 4.6). Differences existed in significant nodes across groups and between depths. 
The primary TKA group represents the native response, as these tissues are not in contact with implants or infection that cause persistent inflammation [51,52]. In primary TKA, all significant nodes in the ATL had low radiality values, and all four were cytokines: IL- $1 \alpha$, IL-1 $\beta$, IL-6, and IL-10. Within this network, these cytokines appear to be acting as regulatory molecules. IL-1 $\alpha$, IL-1 $\beta$, and IL-6 are all pro-inflammatory cytokines vital for early inflammatory immune response [6,8]. The anti-inflammatory IL-10 is central for wound resolution [54]. In the RTL of primary TKA, nodes with significant low radiality values were p-HSP27, p-AKT, p-ERK1/2, IL$1 \alpha$, IL-1 $\beta$, and IL-6 (Tables 4.5 and 4.6). This suggests that there is still a significant contribution of pro-inflammatory cytokines in healthy tissues spatially removed from the joint. p-HSP27, p$\mathrm{AKT}$, and p-ERK1/2 have all been linked to early proliferative wound healing responses in trauma [55] and skin wounds [56]. Their low radiality outcomes suggested that these three phosphoproteins may be driving the tissue healing response. Additionally, in the RTL of primary TKA, seven phosphoproteins had significantly high radiality values (Table 4.6), suggesting that there is an organized wound healing response in tissues further away from the joint.

The aseptic and septic TKR groups were compared to the primary TKA group to understand differences in radiality outcomes. In the aseptic ATL, nodes with significant low radiality outcomes were p-CREB, p-c-Jun, p-BAD, IL-6, IL-10, IL-12p70, and IL-13. Additionally, eight phosphoproteins and one cytokine had significant high radiality outcomes (Table 4.5). Overall, in the ATL of aseptic TKR, there appears to be a balance of regulated and dysregulated healing processes. In combination with the IPA network results, this suggested that dysregulation may be caused by reduced contributions for pro-inflammatory IL-1 $\alpha$ and IL-1 $\beta$ and an increased role for anti-inflammatory IL-13 between primary TKA response and aseptic TKR response at the 
joint (Figure 4.6). The pro-apoptotic actions of peripheral p-c-Jun and p-BAD [33,39], and inactivation of CREB [57] in aseptic TKR could also be driving these disruptions (Figures 4.64.7). In the RTL of aseptic TKR, p-CREB, p-BAD, IL-10, IL-13, and IFN- $\gamma$ gave significant low radiality outcomes (Table 4.6). The aseptic RTL tissues showed a shift to all significant nodes showing low radiality outcomes (Table 4.6). Compared to the primary RTL, there is a notable induction of anti-inflammatory cytokines IL-10, IL-13, and IFN- $\gamma$. Significance of p-CREB and p-BAD suggested these activated proteins may be promoting apoptosis $[30,39]$ in presumably healthy aseptic tissues. Further, the coordinated healing response observed in primary RTL tissues is no longer present, as there were no significant high radiality outcomes in aseptic RTL (Table 4.6). Even in the clinically "healthy" tissues for the aseptic group, there is a large amount of dysregulation present, and it appears to be primarily driven by these seven targets: p-c-Jun, pCREB, p-BAD, IL-10, IL-12p70, IL-13, and IFN- $\gamma$.

In septic TKR, nodes with significant low radiality outcomes in the ATL were p-PTEN, IL-1 $\alpha$, IL-1 $\beta$, IL-4, IL-6, IL-13, IFN- $\gamma$, and TNF- $\alpha$. Notably, there were no significant high radiality outcomes (Table 4.5). While this may somewhat reflect the strong cytokine-dependent response observed in primary TKA, differences include increased contributions of antiinflammatory IL-4, IL-13, and IFN- $\gamma$, pro-inflammatory TNF- $\alpha$, and pro-apoptotic p-PTEN in the septic TKR group. The ATL of septic TKR showed a notable induction of anti-inflammatory cytokines not observed in the primary TKA. In the septic RTL layer, IL-10, IL-13, IFN- $\gamma$, and TNF- $\alpha$ gave significant low radiality values. There were no significant outcomes with high radiality values in this network (Table 4.6). Additionally, there was no overlap in significant low radiality targets between primary TKA and septic TKR tissues at the RTL depth. This loss of 
centrality for wound healing targets in the "healthy" septic TKR tissues reflects a disruption in normal response.

There were some limitations to the study. A single surgeon collected all tissue samples for the cohort of patients involved. Treatment of PJI via debridement is a subjective assessment of tissue viability [28], so the delineation between "healthy" and "unhealthy" tissues may vary between surgeons. The results for the RTL depths of aseptic and septic TKR highlighted the disruptions still present in presumably healthy tissues removed from the joint, so a larger cohort of patients from different surgeons may aid future studies in analyzing these targets. Further, it is difficult to fully disentangle the inflammation present in native response from chronic inflammation and infection. The primary TKA group is expected to experience inflammation as a result of the surgery [58], which is why this study focused on outlining the differences between groups. These differences may not account for all inflammation occurring in the tissues, but the discrepancies between targets may help identify the dysregulation observed in aseptic and septic TKR. Differences in tissue composition (including bone, cartilage, and synovium) may also have played a role in introducing variability between cytokine and phosphoprotein levels; this study focused on including the most likely tissues taken from debridement, regardless of composition. Finally, the IPA analysis was only qualitatively useful in this case due to experimental constraints. While IPA can be used quantitatively for proteomics [59], the samples must be normalized to a control group. The primary TKA is not a true control, only a comparative group. In human subjects, we cannot ethically collect a true tissue control (i.e., healthy individuals with no inflammation present), which limited our ability to analyze via IPA. However, the qualitative comparison at the 
joint still supported the network centrality analysis, and the IPA provided some confirmation of the roles of the targets involved.

\subsection{Conclusion}

The acute intra- and extracellular responses to infection have been studied extensively, and these studies have provided valuable information for clinicians to develop diagnostics and therapeutics to combat these infections [50]. However, less is known about the dysregulation that occurs when inflammation and infections become chronic, which is the case in localized infections like PJI [12]. In this study, we aimed to define the impact of individual cytokines and phosphoproteins on chronic inflammation and infection in PJI using a network centrality parameter approach. Overall, network centrality analysis showed the native response in primary TKA tissues was dictated by a balance of pro- and anti-inflammatory cytokines. Tissues in the ATL were highly influenced by pro-inflammatory cytokines IL- $1 \alpha$, IL-1 $\beta$, and IL- 6 and anti-inflammatory IL-10. A variety of pro-inflammatory cytokines and wound healing phosphoproteins were central to the network in the RTL, and this response was reflective of normal tissue healing processes $[8,30,43]$. Deviations from this response were observed in both aseptic and septic TKR groups. In aseptic TKR tissues, a shift to increased peripheral roles for pro-apoptotic and anti-inflammatory targets was prevalent at both ATL and RTL tissue depths. In the septic ATL layer, pro-apoptotic p-PTEN and anti-inflammatory cytokines IL-4, IL-13, and IFN- $\gamma$ showed significant losses of centrality compared to primary TKA. The high contributions of nodes with seemingly contradictory roles, combined with the loss of overall IPA network connectivity, highlights the dysregulation near the joint in septic TKR tissues. At the septic RTL depth, anti-inflammatory cytokines dominated the response, showing a hallmark absence of coordinated phosphoproteins linked to wound healing. 
The radiality data as a whole suggested that disrupted signaling pathways are present for both aseptic and septic TKR, even in presumably "healthy" tissues. Targeting the proteins with significant radiality outcomes in chronic inflammation and infection may prove useful for developing more effective therapeutics, and future studies should focus on these proteins to promote tissue healing and infection resolution in PJI.

\subsection{Acknowledgements}

Funding for this work was provided by the National Institutes of Health [K08AR073921], and institutional support provided by the West Virginia University School of Medicine, Department of Orthopaedics. The authors would like to thank Jennifer Eicher for her work in recruitment of patients for the study. Further, we would like to thank Suzanne Danley for her editorial suggestions. We would like to thank Clara Warner for developing graphic design images of the knee anatomy.

\subsection{References}

[1] Arango Duque G, Descoteaux A. Macrophage cytokines: involvement in immunity and infectious diseases. Front Immunol. 2014;5:491. doi:10.3389/fimmu.2014.00491

[2] Chaudhry H, Zhou J, Zhong Y, et al. Role of cytokines as a double-edged sword in sepsis. In Vivo. 2013 Nov-Dec 2013;27(6):669-84.

[3] Cavaillon JM, Adib-Conquy M, Fitting C, Adrie C, Payen D. Cytokine cascade in sepsis. Scand J Infect Dis. 2003;35(9):535-44. doi:10.1080/00365540310015935 
[4] Richter E, Mostertz J, Hochgräfe F. Proteomic discovery of host kinase signaling in bacterial infections. Proteomics Clin Appl. 10 2016;10(9-10):994-1010. doi:10.1002/prca.201600035

[5] Zhang H, Sun J, Ye J, et al. Quantitative Label-Free Phosphoproteomics Reveals Differentially Regulated Protein Phosphorylation Involved in West Nile Virus-Induced Host Inflammatory Response. J Proteome Res. Dec 2015;14(12):5157-68. doi:10.1021/acs.jproteome.5b00424

[6] Ma L, Zhang H, Yin YL, et al. Role of interleukin-6 to differentiate sepsis from noninfectious systemic inflammatory response syndrome. Cytokine. 12 2016;88:126-135. doi:10.1016/j.cyto.2016.08.033

[7] Lopez-Castejon G, Brough D. Understanding the mechanism of IL-1 $\beta$ secretion. Cytokine Growth Factor Rev. Aug 2011;22(4):189-95. doi:10.1016/j.cytogfr.2011.10.001

[8] Dinarello CA. Overview of the IL-1 family in innate inflammation and acquired immunity. Immunol Rev. 01 2018;281(1):8-27. doi:10.1111/imr.12621

[9] Seah CC, Phillips TJ, Howard CE, et al. Chronic wound fluid suppresses proliferation of dermal fibroblasts through a Ras-mediated signaling pathway. J Invest Dermatol. Feb 2005;124(2):466-74. doi:10.1111/j.0022-202X.2004.23557.x

[10] Kaminska B. MAPK signalling pathways as molecular targets for anti-inflammatory therapy--from molecular mechanisms to therapeutic benefits. Biochim Biophys Acta. Dec 2005;1754(1-2):253-62. doi:10.1016/j.bbapap.2005.08.017

[11] Rämet M, Lanot R, Zachary D, Manfruelli P. JNK signaling pathway is required for efficient wound healing in Drosophila. Dev Biol. Jan 2002;241(1):145-56. doi:10.1006/dbio.2001.0502 
[12] Brady RA, Mocca CP, Plaut RD, Takeda K, Burns DL. Comparison of the immune response during acute and chronic Staphylococcus aureus infection. PLoS One. 2018;13(3):e0195342. doi:10.1371/journal.pone.0195342

[13] Li C, Renz N, Trampuz A. Management of Periprosthetic Joint Infection. Hip Pelvis. Sep 2018;30(3):138-146. doi:10.5371/hp.2018.30.3.138

[14] Seebach E, Kubatzky KF. Chronic Implant-Related Bone Infections-Can Immune Modulation be a Therapeutic Strategy? Front Immunol. 2019;10:1724. doi:10.3389/fimmu.2019.01724

[15] Lüthje FL, Jensen LK, Jensen HE, Skovgaard K. The inflammatory response to bone infection - a review based on animal models and human patients. APMIS. Apr 2020;128(4):275286. doi:10.1111/apm.13027

[16] Grant SS, Hung DT. Persistent bacterial infections, antibiotic tolerance, and the oxidative stress response. Virulence. May 2013;4(4):273-83. doi:10.4161/viru.23987

[17] Tande AJ, Patel R. Prosthetic joint infection. Clin Microbiol Rev. Apr 2014;27(2):302-45. doi:10.1128/CMR.00111-13

[18] Kalore NV, Gioe TJ, Singh JA. Diagnosis and management of infected total knee arthroplasty. Open Orthop J. Mar 2011;5:86-91. doi:10.2174/1874325001105010086

[19] Dyskova T, Kriegova E, Slobodova Z, et al. Inflammation time-axis in aseptic loosening of total knee arthroplasty: A preliminary study. PLoS One. 2019;14(8):e0221056. doi:10.1371/journal.pone.0221056

[20] Morel PA, Lee REC, Faeder JR. Demystifying the cytokine network: Mathematical models point the way. Cytokine. 10 2017;98:115-123. doi:10.1016/j.cyto.2016.11.013 
[21] Thomas S, Bonchev D. A survey of current software for network analysis in molecular biology. Hum Genomics. Jun 2010;4(5):353-60. doi:10.1186/1479-7364-4-5-353

[22] van Riel NA. Dynamic modelling and analysis of biochemical networks: mechanism-based models and model-based experiments. Brief Bioinform. Dec 2006;7(4):364-74. doi:10.1093/bib/bbl040

[23] Scardoni G, Laudanna C. Centralities Based Analysis of Complex Networks. In: Zhang Y, ed. New Frontiers in Graph Theory. Intech Open; 2012.

[24] Abedi M, Gheisari Y. Nodes with high centrality in protein interaction networks are responsible for driving signaling pathways in diabetic nephropathy. PeerJ. 2015;3:e1284. doi:10.7717/peerj.1284

[25] Peng Q, Schork NJ. Utility of network integrity methods in therapeutic target identification. Front Genet. 2014;5:12. doi:10.3389/fgene.2014.00012

[26] DeBoer J, Jagadish T, Haverland NA, Madson CJ, Ciborowski P, Belshan M. Alterations in the nuclear proteome of HIV-1 infected T-cells. Virology. Nov 2014;468-470:409-420. doi:10.1016/j.virol.2014.08.029

[27] Parvizi J, Tan TL, Goswami K, et al. The 2018 Definition of Periprosthetic Hip and Knee Infection: An Evidence-Based and Validated Criteria. J Arthroplasty. 05 2018;33(5):1309-1314.e2. doi:10.1016/j.arth.2018.02.078

[28] Qasim SN, Swann A, Ashford R. The DAIR (debridement, antibiotics and implant retention) procedure for infected total knee replacement - a literature review. SICOT J. 2017;3:2. doi:10.1051/sicotj/2016038 
[29] Hulse RE, Kunkler PE, Fedynyshyn JP, Kraig RP. Optimization of multiplexed bead-based cytokine immunoassays for rat serum and brain tissue. J Neurosci Methods. Jun 2004;136(1):8798. doi:10.1016/j.jneumeth.2003.12.023

[30] Couture C, Desjardins P, Zaniolo K, Germain L, Guérin SL. Enhanced wound healing of tissue-engineered human corneas through altered phosphorylation of the CREB and AKT signal transduction pathways. Acta Biomater. 06 2018;73:312-325. doi:10.1016/j.actbio.2018.04.021

[31] Atalay M, Oksala N, Lappalainen J, Laaksonen DE, Sen CK, Roy S. Heat shock proteins in diabetes and wound healing. Curr Protein Pept Sci. Feb 2009;10(1):85-95. doi:10.2174/138920309787315202

[32] Reid MB, Li YP. Cytokines and oxidative signalling in skeletal muscle. Acta Physiol Scand. Mar 2001;171(3):225-32. doi:10.1046/j.1365-201x.2001.00824.x

[33] Yang M, Guan DW, Xiong CY, Cheng ZH, Yu TS. [Expression of c-jun during the incised wound healing in mice skin]. Fa Yi Xue Za Zhi. Dec 2009;25(6):401-4.

[34] Hosokawa R, Urata MM, Ito Y, Bringas P, Chai Y. Functional significance of Smad2 in regulating basal keratinocyte migration during wound healing. J Invest Dermatol. Dec 2005;125(6):1302-9. doi:10.1111/j.0022-202X.2005.23963.x

[35] Nédellec S, Renaudineau Y, Bordron A, et al. B cell response to surface IgM cross-linking identifies different prognostic groups of B-chronic lymphocytic leukemia patients. J Immunol. Mar 2005;174(6):3749-56. doi:10.4049/jimmunol.174.6.3749

[36] Vollmar B, El-Gibaly AM, Scheuer C, Strik MW, Bruch HP, Menger MD. Acceleration of cutaneous wound healing by transient p53 inhibition. Lab Invest. Aug 2002;82(8):1063-71. doi:10.1097/01.lab.0000024363.37866.45 
[37] Matsubayashi Y, Ebisuya M, Honjoh S, Nishida E. ERK activation propagates in epithelial cell sheets and regulates their migration during wound healing. Curr Biol. Apr 2004;14(8):731-5. doi:10.1016/j.cub.2004.03.060

[38] Zhao M. PTEN: a promising pharmacological target to enhance epithelial wound healing. Br J Pharmacol. Dec 2007;152(8):1141-4. doi:10.1038/sj.bjp.0707503

[39] Downward J. How BAD phosphorylation is good for survival. Nat Cell Biol. Jun 1999;1(2):E33-5. doi:10.1038/10026

[40] Lin L, White SA, Hu K. Role of p90RSK in Kidney and Other Diseases. Int J Mol Sci. Feb 2019;20(4)doi:10.3390/ijms20040972

[41] Bao P, Kodra A, Tomic-Canic M, Golinko MS, Ehrlich HP, Brem H. The role of vascular endothelial growth factor in wound healing. J Surg Res. May 2009;153(2):347-58. doi:10.1016/j.jss.2008.04.023

[42] Zhang J, Bárdos T, Shao Q, et al. IL-4 potentiates activated T cell apoptosis via an IL-2dependent mechanism. J Immunol. Apr 2003;170(7):3495-503. doi:10.4049/jimmunol.170.7.3495 [43] Matias MA, Saunus JM, Ivanovski S, Walsh LJ, Farah CS. Accelerated wound healing phenotype in Interleukin 12/23 deficient mice. J Inflamm (Lond). Dec 2011;8:39. doi:10.1186/1476-9255-8-39

[44] Kaviratne M, Hesse M, Leusink M, et al. IL-13 activates a mechanism of tissue fibrosis that is completely TGF-beta independent. J Immunol. Sep 2004;173(6):4020-9. doi:10.4049/jimmunol.173.6.4020

[45] Li A, Feng M, Li Y, Liu Z. Application of Outlier Mining in Insider Identification Based on Boxplot Method. Procedia Computer Science. 2016;91:245-251. doi:10.1016/j.procs.2016.07.069 
[46] Bland JM, Altman DG. Multiple significance tests: the Bonferroni method. BMJ. Jan 1995;310(6973):170. doi:10.1136/bmj.310.6973.170

[47] Ghosh S, Zang S, Mitra PS, Ghimbovschi S, Hoffman EP, Dutta SK. Global gene expression and Ingenuity biological functions analysis on PCBs 153 and 138 induced human PBMC in vitro reveals differential mode(s) of action in developing toxicities. Environ Int. Jul 2011;37(5):838-57. doi:10.1016/j.envint.2011.02.010

[48] Bonnet A, Lagarrigue S, Liaubet L, Robert-Granié C, Sancristobal M, Tosser-Klopp G. Pathway results from the chicken data set using GOTM, Pathway Studio and Ingenuity softwares. BMC Proc. Jul 2009;3 Suppl 4:S11. doi:10.1186/1753-6561-3-S4-S11

[49] Han AA, Currie HN, Loos MS, et al. Spatiotemporal phosphoprotein distribution and associated cytokine response of a traumatic injury. Cytokine. Mar 2016;79:12-22. doi:10.1016/j.cyto.2015.12.006

[50] Monastero RN, Pentyala S. Cytokines as Biomarkers and Their Respective Clinical Cutoff Levels. Int J Inflam. 2017;2017:4309485. doi:10.1155/2017/4309485

[51] Heim CE, Vidlak D, Scherr TD, Hartman CW, Garvin KL, Kielian T. IL-12 promotes myeloid-derived suppressor cell recruitment and bacterial persistence during Staphylococcus aureus orthopedic implant infection. J Immunol. Apr 2015;194(8):3861-3872. doi:10.4049/jimmunol.1402689

[52] Anderson JM, McNally AK. Biocompatibility of implants: lymphocyte/macrophage interactions. Semin Immunopathol. May 2011;33(3):221-33. doi:10.1007/s00281-011-0244-1

[53] Gawel DR, Serra-Musach J, Lilja S, et al. A validated single-cell-based strategy to identify diagnostic and therapeutic targets in complex diseases. Genome Med. 07 2019;11(1):47. doi:10.1186/s13073-019-0657-3 
[54] Peranteau WH, Zhang L, Muvarak N, et al. IL-10 overexpression decreases inflammatory mediators and promotes regenerative healing in an adult model of scar formation. J Invest Dermatol. Jul 2008;128(7):1852-60. doi:10.1038/sj.jid.5701232

[55] Han AA, Currie HN, Loos MS, et al. The impact of cytokine responses in the intra- and extracellular signaling network of a traumatic injury. Cytokine. 06 2018;106:136-147. doi:10.1016/j.cyto.2017.10.027

[56] Lee S, Kim MS, Jung SJ, Kim D, Park HJ, Cho D. ERK activating peptide, AES16-2M promotes wound healing through accelerating migration of keratinocytes. Sci Rep. 09 2018;8(1):14398. doi:10.1038/s41598-018-32851-y

[57] François F, Godinho MJ, Grimes ML. CREB is cleaved by caspases during neural cell apoptosis. FEBS Lett. Dec 2000;486(3):281-4. doi:10.1016/s0014-5793(00)02316-4

[58] Mayer C, Franz A, Harmsen JF, et al. Soft-tissue damage during total knee arthroplasty: Focus on tourniquet-induced metabolic and ionic muscle impairment. J Orthop. Sep 2017;14(3):347-353. doi:10.1016/j.jor.2017.06.015

[59] Kamal AHM, Fessler MB, Chowdhury SM. Comparative and network-based proteomic analysis of low dose ethanol- and lipopolysaccharide-induced macrophages. PLoS One. 2018;13(2):e0193104. doi:10.1371/journal.pone.0193104 


\section{Chapter 5}

Conclusions and Future Directions: Translating Signal Transduction Research into Clinically-Relevant Tools 
The work presented in this dissertation was aimed towards improving the understanding of chronic, localized infections through analysis of signal transduction pathways and represents one example of a basic research approach to a clinical problem, chronic infection. It began with an investigation of a clinical cohort to identify biomarkers of chronic PJI at two spatial gradients, then moved onto further probing of upstream and downstream effects of infection using an in vivo model. Finally, it culminated with validation of network centrality radiality analysis of signal mediators to understand drivers of disrupted responses observed in the clinical and in vivo studies. It represented a novel approach to prospective, tissue-level investigations of cell signaling changes in response to inflammatory stimuli, and the analyses demonstrated that distinct inflammatory immune and wound healing profiles exist in response to implants and chronic implant infections compared to native tissues. Based on this work, it was clear that both implants and chronic joint infections provoke cell signaling changes that negatively impact immune and wound healing responses on a tissue level.

This research laid a foundation to better understand the tissue-level response to chronic infection, but there is more work to be done before it can be translated into the clinic. The final chapter of this dissertation will discuss important next steps for this work and implications for similar investigations. This work was the initial attempt to investigate the implications of tissuelevel disruptions caused by chronic infection (e.g., tissue viability), and the use of 'omics technologies and newly emerging analytical techniques promise exciting progress in follow-up investigations of these signal transduction networks. The success in translation of this signal transduction work into the clinic will rely on comprehensive analyses of mediators involved, use of data integration techniques, and compatibility with expeditious results and interpretation. 


\subsection{Localized vs. Systemic Investigations of Clinical Pathologies}

An important consideration for signal transduction studies is whether to focus efforts on the localized response in individual organs or tissues or the systemic response in blood and other biological samples. The research goals of the study dictate the correct choice, and a variety of studies have investigated relationships between localized and systemic responses in disease states. Systemic measurements can prove beneficial for a variety of non-invasive applications, such as point-of-care diagnostics, in which samples can easily be acquired from blood or other fluids with minimal processing, providing a rapid means of obtaining clinical information. Studies concerned with infection or sepsis have concluded that both local and systemic information can be useful, and immune-related factors- such as cytokines- have been utilized as biomarkers on either level. Monitoring circulating levels of immune-related cytokines has proven useful for a range of clinically-relevant applications, including determining the presence of infection [1,2], monitoring risk and severity of sepsis [3], and differentiating between types of infections [4, 5]. Systemic levels of immune mediators have also been pursued for other pathological conditions. Serum biomarkers have been identified in cancers to monitor drug efficacy [6] or predict adverse outcomes [7]. Concentrations of extracellular matrix proteins in serum have shown utility for monitoring tissue healing in chronic obstructive pulmonary disorder (COPD) [8]. Even tears [9] and saliva [10] have been used as mediums to better understand the immune response to stress and disease. Most of these systemic measurements are best suited for monitoring immune response over time or predicting adverse outcomes.

Localized investigations can highlight the ability of specific organs or tissue types to counter immune-related insults like infection, and these responses may differ with proximity, as 
demonstrated in Chapters 2 and 3. Different tissues are composed of different cell types, and variability of responses may arise due to tissue composition, further highlighting the importance of considering a localized perspective for signal transduction research. Several studies have demonstrated differences between local and systemic immune environments [11], and localized profiles of immune mediators have been investigated in models of infection to understand the tissue-specific response $[12,13]$, including the work presented in this dissertation. Localized investigations have also been pursued for other conditions, and Hauser et al. demonstrated that the immune microenvironment differs between localized and circulating levels in response to human fracture and soft-tissue trauma [14]. Similarly, Currie et al. characterized the spatial gradients of immune and wound healing factors in a traumatic injury model in rodents to show that proximity dictates the response $[15,16]$. Differences in localized and systemic profiles were investigated in organ failure with similar conclusions, that these localized perspectives offered tissue-specific insights relevant to disease management [17]. These studies illustrated that these responses might vary considerably depending on tissue type, and a localized approach is necessary to understand the specific responses of tissues and the effects on signal transduction cascades that arise following these insults. The spatial investigation conducted in this work demonstrated that spatial gradients exist in the tissue-level response to implants and infection, and tissues closer to the joint showed higher concentrations of cytokines and higher degrees of protein phosphorylation, so proximity is an important factor in the immune and wound healing responses. In the future, a comparison of serum would inform clinicians of systemic-level inflammation, which is likely higher in patients with aseptic and septic joints and could have negative implications for overall health. Understanding the systemic effects of joint implants and joint infections is an important consideration for Orthopaedists, but has not yet been addressed. 
Depending on an individual study's intended outcomes, different spatial approaches may be suitable. Systemic responses are beneficial for biomarker identification and non-invasive monitoring of disease states, but studying localized responses in individual tissues or organs will be critical to making clinical assessments of tissue health (e.g., debridement) more objective. A localized approach was essential in this work to create a network of tissue-level responses to best understand the response to infection, and similar studies may benefit when aiming to identify healthy versus non-healthy tissues in response to stress, insults, and other diseases.

\subsection{Integrating Multi-'Omics Data to Approach Clinical Problems}

Probing biological networks on a tissue level in response to infection elucidated the interactions of highly interconnected targets, resulting in improved knowledge of the drivers of signaling and the potential points of disruption. In Chapter 4, changes in networks resulting from implant and infection were analyzed; Using network centrality radiality, it became possible to identify which targets were likely driving the dysregulated response observed in these inflammatory states. A thorough investigation of biological networks using mathematical modeling is a relatively new analysis strategy in developing improved diagnostic and therapeutics for many diseases $[18,19]$. These network approaches often involve analyzing many targets simultaneously, and these data can be acquired from multiple 'omics platforms. This creates a situation in which there are many more variables being tested than the number of replicates in the study, which has introduced new challenges for interpretation.

Researchers must carefully consider what type of data to acquire and how best to integrate different data sources to support their hypothesis. To fully understand a disease or insult, it may 
be necessary to investigate genetic factors, bioenergetics, immune response, or other elements. While acquiring more data is often beneficial, it has become essential to develop new methods to deal with these large data sets [20-22]. Translating multi-'omics data into clinically-relevant information is the next challenge in identifying markers of tissue health in chronic infection, and these strategies could be applied to other investigations of cell signaling disruptions relevant to disease. While the initial research into tissue health in chronic infection was pursued in Chapter 4 using network centrality, implementing other 'omics techniques in the future- like metabolomics and transcriptomics- could better inform the distinction between healthy and non-healthy tissue, which is essential to develop useful clinical tools.

Investigating metabolomic profiles related to implants and chronic infection is the logical next step towards improving tissue-level treatment. The cytokine and phosphoprotein data showed shifts in tissue responses, but they did not fully explain the implications for tissue health. Cellular metabolism changes are intimately linked to inflammatory response [30], and altered metabolite profiles have been observed in response to inflammatory stimuli [31]. Studies of polar metabolites have been used in literature to identify specific disruptions for a variety of pathological conditions [32], as many polar metabolites play key roles in growth, development, and conversion of nutrients. Targeting changes in these compounds could enlighten the distinct effects of chronic infection on these wound healing-related processes. Metabolomic analysis coupled to previously collected proteomic data on cytokines and phosphoproteins provides a means for pinpointing the specific infection-related disruptions between aseptic and septic joints. Based on literature investigations of skeletal muscle in other disease states, the prevailing hypothesis is that tissues responding to implants and infection will show lower levels of important polar metabolites related to energy 
consumption (e.g., glucose, lactose). This shift will reflect the altered cellular states resulting from chronic exposure to inflammatory stimuli. Long-term metabolic health of these tissues is of the utmost importance to surgeons tasked with removing unhealthy and non-viable tissues.

\subsection{New Technologies for Rapid Analysis}

While many techniques are available to acquire data through multi-'omics platforms, clinical relevance is dependent on ease of acquisition and interpretation. Biological network analysis of signal transduction networks is a complicated field, and researchers must keep this in mind when seeking to translate their findings into clinically-relevant information. The work presented in Chapters 2-4 heavily relied on multiplexed ELISA for data acquisition, but these assays required 4-20 hours for data acquisition alone. While the specificity of ELISA techniques was beneficial to lay the groundwork to understand targets of interest, this approach would not be appropriate for intra-operative use to treat these chronic infections, so future research should investigate the use of rapid analytical techniques to achieve this goal.

Advances in the field, such as rapid ELISA platforms [23, 24], miniature field-applicable mass spectrometry [25], and other point-of-care testing have opened doors to advance the science of biomarker detection [26, 27], and these platforms will prove essential to creating clinicallyrelevant means of analysis. While basic science investigations, like the work presented here, can initially be used to identify markers of interest for disease, the ultimate goal is to provide a rapid, sensitive platform for acquisition with results that can be easily interpreted in the clinic. Future work to understand tissue viability in chronic infection or other disease states will benefit from 
utilizing these newly emerging analytical tools to provide this information quickly. Shotgun metagenomic sequencing has been explored for fast identification of bacterial infection in synovial fluids in infection and showed faster results than traditional ELISAs [28]. Ultrafast qRT-PCR gene profiling coupled to machine learning algorithms has also demonstrated utility in establishing biomarkers of tissue health, and a study by Fillerova et al. represents one of the few attempts to characterize infection networks directly on a tissue level [29]. While this study included a smaller cohort, researchers achieved $94.5 \%$ sensitivity and $95.7 \%$ specificity for infection diagnosis using their algorithm and could provide diagnostic results within 45 minutes of sample collection.

The future of signal transduction-focused research to understand chronic inflammation and infection will benefit from comparing local vs. systemic measurements and incorporating multi'omics strategies to understand tissue health. Enrolling larger cohorts to include patients from multiple surgeons is the next step to tackling these problems, allowing a broader spectrum of expertise to delineate between healthy and unhealthy tissues and providing more tissue samples dedicated to other 'omics platforms. Additional animal studies should be performed to investigate the temporal progression of immune and wound healing dysfunctions via transcriptomic and metabolomic analyses, which could provide insight into the transition from acute to chronic inflammatory states and enhance understanding of overall tissue viability relevant to surgical debridement. Rapid analytical platforms will be critical in developing clinical tools from the biomarkers identified in these studies to combat chronic inflammation and infection in the future. Approaching these clinically-relevant problems through signal transduction network investigation is key to improving current treatment methods, and the strategies utilized in these studies could be applied to other disease states. 


\subsection{References}

[1] Monastero RN, Pentyala S: Cytokines as Biomarkers and Their Respective Clinical Cutoff Levels. Int J Inflam 2017, 2017:4309485.

[2] Tang Y, Liao C, Xu X, Song H, Shi S, Yang S, Zhao F, Xu W, Chen X, Mao J, et al: Evaluation of Th1/Th2 cytokines as a rapid diagnostic tool for severe infection in paediatric haematology/oncology patients by the use of cytometric bead array technology. Clin Microbiol Infect 2011, 17:1666-1673.

[3] Ye Q, Shao WX, Xu XJ, Yang YZ: The clinical application value of cytokines in treating infectious diseases. PLoS One 2014, 9:e98745.

[4] Su WL, Perng WC, Huang CH, Yang CY, Wu CP, Chang FY, Chen JH: Identification of cytokines in whole blood for differential diagnosis of tuberculosis versus pneumonia. Clin Vaccine Immunol 2010, 17:771-777.

[5] Wang S, Li Y, Shen Y, Wu J, Gao Y, Zhang S, Shao L, Jin J, Zhang Y, Zhang W: Screening and identification of a six-cytokine biosignature for detecting $\mathrm{TB}$ infection and discriminating active from latent TB. J Transl Med 2018, 16:206.

[6] Linder S, Alaiya A: Serum efficacy biomarkers for oncology. Biomark Med 2009, 3:4754.

[7] Kiebish MA, Cullen J, Mishra P, Ali A, Milliman E, Rodrigues LO, Chen EY, Tolstikov V, Zhang L, Panagopoulos K, et al: Multi-omic serum biomarkers for prognosis of disease progression in prostate cancer. J Transl Med 2020, 18:10.

[8] Stolz D, Leeming DJ, Kristensen JHE, Karsdal MA, Boersma W, Louis R, Milenkovic B, Kostikas K, Blasi F, Aerts J, et al: Systemic Biomarkers of Collagen and Elastin Turnover Are Associated With Clinically Relevant Outcomes in COPD. Chest 2017, 151:47-59. 
[9] von Thun Und Hohenstein-Blaul N, Funke S, Grus FH: Tears as a source of biomarkers for ocular and systemic diseases. Exp Eye Res 2013, 117:126-137.

[10] Newton TL, Fernandez-Botran R, Lyle KB, Szabo YZ, Miller JJ, Warnecke AJ: Salivary cytokine response in the aftermath of stress: An emotion regulation perspective. Emotion 2017, 17:1007-1020.

[11] Kather JN, Halama N: Harnessing the innate immune system and local immunological microenvironment to treat colorectal cancer. Br J Cancer 2019, 120:871-882.

[12] Chin VK, Foong KJ, Maha A, Rusliza B, Norhafizah M, Chong PP: Early expression of local cytokines during systemic. Biomed Rep 2014, 2:869-874.

[13] Czepiel J, Biesiada G, Brzozowski T, Ptak-Belowska A, Perucki W, Birczynska M, Jurczyszyn A, Strzalka M, Targosz A, Garlicki A: The role of local and systemic cytokines in patients infected with Clostridium difficile. J Physiol Pharmacol 2014, 65:695-703.

[14] Hauser CJ, Zhou X, Joshi P, Cuchens MA, Kregor P, Devidas M, Kennedy RJ, Poole GV, Hughes JL: The immune microenvironment of human fracture/soft-tissue hematomas and its relationship to systemic immunity. J Trauma 1997, 42:895-903; discussion 903-894.

[15] Han AA, Currie HN, Loos MS, Vrana JA, Fabyanic EB, Prediger MS, Boyd JW: Spatiotemporal phosphoprotein distribution and associated cytokine response of a traumatic injury. Cytokine 2016, 79:12-22.

[16] Currie HN, Loos MS, Vrana JA, Dragan K, Boyd JW: Spatial cytokine distribution following traumatic injury. Cytokine 2014, 66:112-118.

[17] Levi M, van der Poll T, Schultz M: Systemic versus localized coagulation activation contributing to organ failure in critically ill patients. Semin Immunopathol 2012, 34:167179. 
[18] Oulas A, Minadakis G, Zachariou M, Sokratous K, Bourdakou MM, Spyrou GM: Systems Bioinformatics: increasing precision of computational diagnostics and therapeutics through network-based approaches. Brief Bioinform 2019, 20:806-824.

[19] Peng Q, Schork NJ: Utility of network integrity methods in therapeutic target identification. Front Genet 2014, 5:12.

[20] Williams JR, Yang R, Clifford JL, Watson D, Campbell R, Getnet D, Kumar R, Hammamieh R, Jett M: Functional Heatmap: an automated and interactive pattern recognition tool to integrate time with multi-omics assays. BMC Bioinformatics 2019, 20:81.

[21] Pinu FR, Beale DJ, Paten AM, Kouremenos K, Swarup S, Schirra HJ, Wishart D: Systems Biology and Multi-Omics Integration: Viewpoints from the Metabolomics Research Community. Metabolites 2019, 9.

[22] Bersanelli M, Mosca E, Remondini D, Giampieri E, Sala C, Castellani G, Milanesi L: Methods for the integration of multi-omics data: mathematical aspects. BMC Bioinformatics 2016, 17 Suppl 2:15.

[23] Ma L, Nilghaz A, Choi JR, Liu X, Lu X: Rapid detection of clenbuterol in milk using microfluidic paper-based ELISA. Food Chem 2018, 246:437-441.

[24] Schöler L, Le-Trilling VTK, Eilbrecht M, Mennerich D, Anastasiou OE, Krawczyk A, Herrmann A, Dittmer U, Trilling M: A Novel In-Cell ELISA Assay Allows Rapid and Automated Quantification of SARS-CoV-2 to Analyze Neutralizing Antibodies and Antiviral Compounds. Front Immunol 2020, 11:573526.

[25] Ma X, Ouyang Z: Ambient ionization and miniature mass spectrometry system for chemical and biological analysis. Trends Analyt Chem 2016, 85:10-19. 
[26] Kozel TR, Burnham-Marusich AR: Point-of-Care Testing for Infectious Diseases: Past, Present, and Future. J Clin Microbiol 2017, 55:2313-2320.

[27] McRae MP, Simmons GW, Christodoulides NJ, Lu Z, Kang SK, Fenyo D, Alcorn T, Dapkins IP, Sharif I, Vurmaz D, et al: Clinical decision support tool and rapid point-ofcare platform for determining disease severity in patients with COVID-19. Lab Chip 2020, 20:2075-2085.

[28] Huang Z, Li W, Lee GC, Fang X, Xing L, Yang B, Lin J, Zhang W: Metagenomic nextgeneration sequencing of synovial fluid demonstrates high accuracy in prosthetic joint infection diagnostics: mNGS for diagnosing PJI. Bone Joint Res 2020, 9:440-449.

[29] Fillerova R, Gallo J, Radvansky M, Kraiczova V, Kudelka M, Kriegova E: Excellent Diagnostic Characteristics for Ultrafast Gene Profiling of. J Clin Microbiol 2017, 55:26862697.

[30] Dunham-Snary KJ, Wu D, Sykes EA, Thakrar A, Parlow LRG, Mewburn JD, Parlow JL, Archer SL: Hypoxic Pulmonary Vasoconstriction: From Molecular Mechanisms to Medicine. Chest 2017, 151:181-192.

[31] Antti H, Fahlgren A, Näsström E, Kouremenos K, Sundén-Cullberg J, Guo Y, Moritz T, Wolf-Watz H, Johansson A, Fallman M: Metabolic profiling for detection of Staphylococcus aureus infection and antibiotic resistance. PLoS One 2013, 8:e56971.

[32] Zhang A, Sun H, Yan G, Wang P, Wang X: Metabolomics for Biomarker Discovery: Moving to the Clinic. Biomed Res Int 2015, 2015:354671. 


\section{APPENDIX A: CHAPTER 2 SUPPLEMENTARY FIGURES}

Table A. 1. Average cytokine concentrations in the adjacent tissue layer (ATL). Concentrations in the LG, F, T, and PC individual tissue locations were averaged to represent the response in the ATL. Standard error of the mean (SEM) and number of patients in each of the primary TKA, aseptic TKR, and septic TKR groups are also reported.

\begin{tabular}{|c|c|c|c|c|c|c|c|c|c|}
\hline & \multicolumn{3}{|c|}{ Primary TKA } & \multicolumn{3}{c|}{ Aseptic TKR } & \multicolumn{3}{c|}{ Septic TKR } \\
\hline & ATL Avg & SEM & N & ATL Avg & SEM & N & ATL Avg & SEM & N \\
\hline IL-1 $\alpha$ & 1.26 & 0.34 & 6 & 14.25 & 3.96 & 5 & 38.70 & 8.75 & 6 \\
\hline IL-1 $\beta$ & 1.77 & 0.47 & 6 & 5.78 & 1.72 & 5 & 51.87 & 23.97 & 6 \\
\hline IL-6 & 10.53 & 2.87 & 6 & 33.11 & 13.90 & 5 & 763.08 & 401.51 & 6 \\
\hline IL-8 & 8.23 & 2.70 & 6 & 101.01 & 62.20 & 5 & 648.39 & 160.55 & 6 \\
\hline MIP-1 $\alpha$ & 7.35 & 2.76 & 6 & 29.08 & 9.86 & 5 & 105.74 & 43.13 & 6 \\
\hline MIP-1 $\beta$ & 24.12 & 5.36 & 6 & 51.66 & 20.73 & 5 & 130.03 & 49.93 & 6 \\
\hline MCP-1 & 151.55 & 27.13 & 6 & 359.90 & 174.07 & 5 & 475.19 & 195.67 & 6 \\
\hline IL-4 & 7.48 & 1.59 & 6 & 21.04 & 3.78 & 5 & 28.37 & 5.03 & 6 \\
\hline IL-10 & 0.89 & 0.17 & 6 & 8.38 & 0.23 & 5 & 5.71 & 1.38 & 6 \\
\hline IL-12p70 & 6.57 & 1.62 & 6 & 33.21 & 10.88 & 5 & 21.51 & 5.74 & 6 \\
\hline IL-13 & 1.98 & 0.42 & 6 & 10.14 & 2.59 & 5 & 9.52 & 2.65 & 6 \\
\hline IL-17A & 6.04 & 2.43 & 6 & 17.37 & 3.85 & 5 & 20.16 & 2.80 & 6 \\
\hline TNF- $\alpha$ & 18.68 & 3.44 & 6 & 79.12 & 20.42 & 5 & 93.12 & 16.60 & 6 \\
\hline
\end{tabular}

Table A. 2. Average cytokine concentrations in the radial tissue layer (RTL). Concentrations in the LG, F, and T individual tissue locations were averaged to represent the response in the RTL. Standard error of the mean (SEM) and number of patients in each of the primary TKA, aseptic TKR, and septic TKR groups are also reported.

\begin{tabular}{|c|c|c|c|c|c|c|c|c|c|}
\hline & \multicolumn{4}{|c|}{ Primary TKA } & \multicolumn{3}{c|}{ Aseptic TKR } & \multicolumn{3}{c|}{ Septic TKR } \\
\hline & RTL Avg & SEM & N & RTL Avg & SEM & N & RTL Avg & SEM & N \\
\hline IL-1 $\alpha$ & 0.89 & 0.25 & 6 & 8.23 & 1.94 & 5 & 16.15 & 3.29 & 6 \\
\hline IL-1 $\beta$ & 1.80 & 0.47 & 6 & 5.10 & 0.96 & 5 & 13.41 & 5.29 & 6 \\
\hline IL-6 & 6.10 & 1.35 & 6 & 11.58 & 5.31 & 5 & 288.59 & 205.91 & 6 \\
\hline IL-8 & 6.06 & 1.91 & 6 & 46.37 & 21.58 & 5 & 350.06 & 188.02 & 6 \\
\hline MIP-1 $\alpha$ & 8.76 & 3.62 & 6 & 23.76 & 13.68 & 5 & 42.20 & 17.64 & 6 \\
\hline MIP-1 $\beta$ & 17.23 & 3.50 & 6 & 36.52 & 19.98 & 5 & 79.97 & 27.68 & 6 \\
\hline MCP-1 & 59.79 & 11.09 & 6 & 126.70 & 51.58 & 5 & 538.87 & 223.47 & 6 \\
\hline IL-4 & 5.96 & 1.60 & 6 & 17.66 & 2.44 & 5 & 19.60 & 3.40 & 6 \\
\hline IL-10 & 0.88 & 0.19 & 6 & 8.50 & 0.16 & 5 & 7.78 & 1.09 & 6 \\
\hline IL-12p70 & 4.10 & 1.18 & 6 & 24.55 & 7.13 & 5 & 19.66 & 6.39 & 6 \\
\hline IL-13 & 1.61 & 0.31 & 6 & 9.10 & 2.43 & 5 & 9.64 & 2.06 & 6 \\
\hline IL-17A & 4.37 & 1.75 & 6 & 14.90 & 3.88 & 5 & 17.31 & 2.71 & 6 \\
\hline TNF- $\alpha$ & 14.27 & 3.99 & 6 & 61.69 & 14.55 & 5 & 78.48 & 16.21 & 6 \\
\hline
\end{tabular}




\section{CYTOKINE CONCENTRATIONS AT INDIVIDUAL LOCATION- INFECTION- SPECIFIC CYTOKINES}

IL-1 $\alpha$

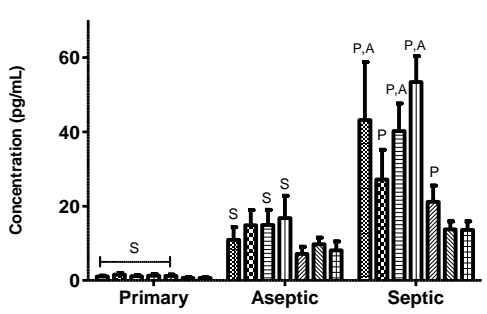

IL-6

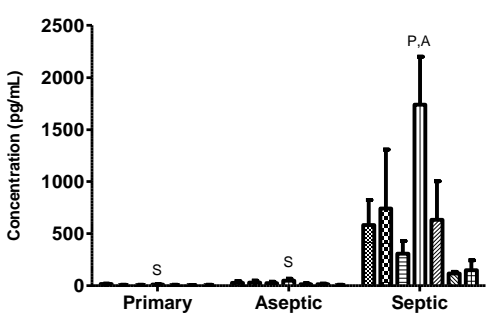

MCP-1

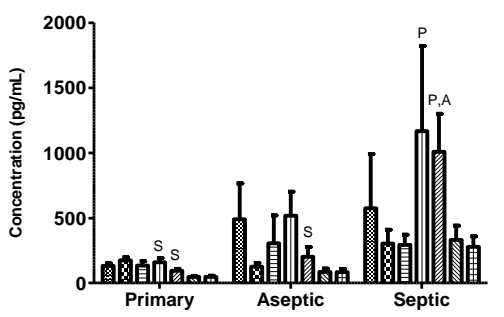

IL-1 $\beta$

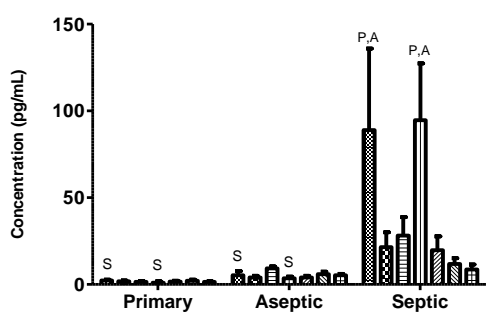

IL-8

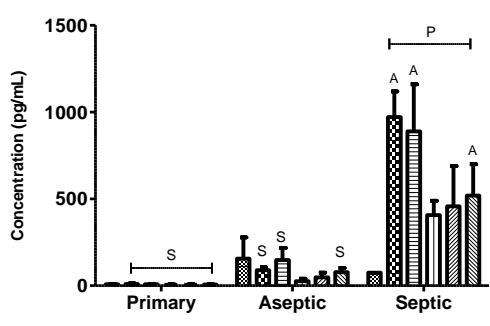

MIP-1 $\alpha$

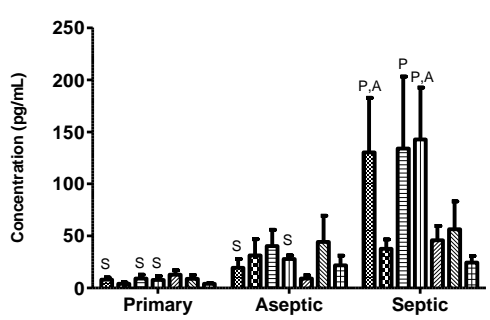

MIP-1 $\beta$

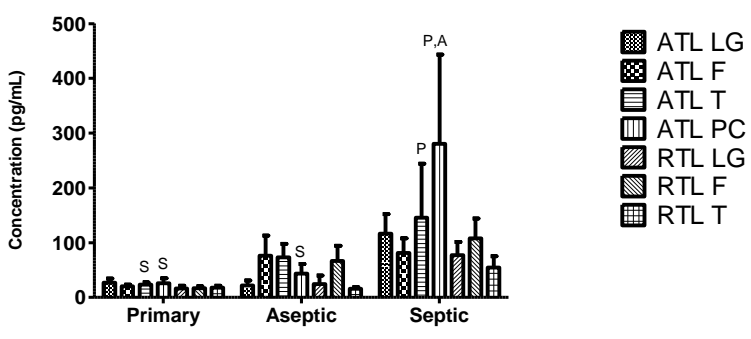

Figure A. 1. Cytokine profiles in localized tissues in infection-specific inflammation. Average cytokine concentration at individual tissue locations are shown for all groups. Two-way ANOVAs with Bonferroni's post-test were conducted to test for significant differences between groups at each tissue depth $(\mathrm{p}<0.05)$. Significant differences between groups at a particular location are marked as: $\mathrm{P}$ denotes significant difference from primary TKA $(\mathrm{N}=6)$; A denotes significant difference from aseptic TKR $(\mathrm{N}=5)$; $\mathrm{S}$ denotes significant difference from septic TKR $(\mathrm{N}=6)$; all symbols denote significance at the $\mathrm{p}<0.05$ level. 


\section{CYTOKINE CONCENTRATIONS AT INDIVIDUAL LOCATION- IMPLANT- RELATED CYTOKINES}

IL-10

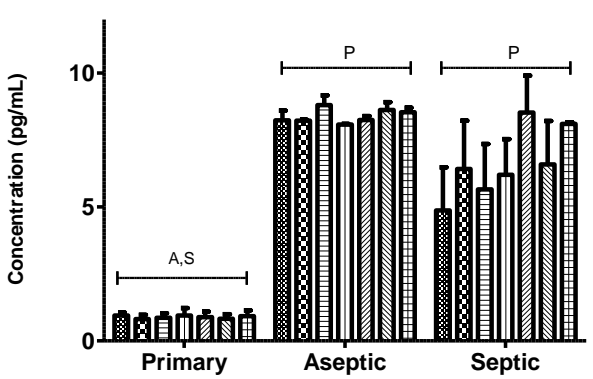

IL-13

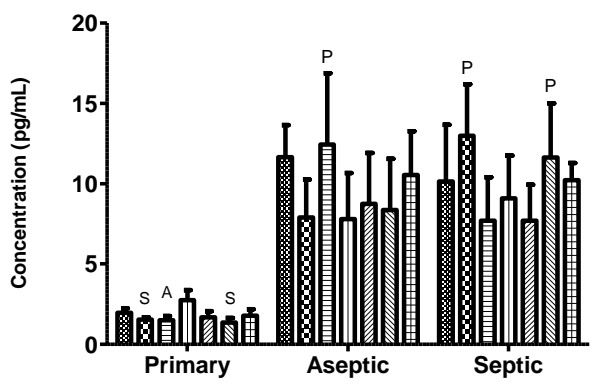

IL-4

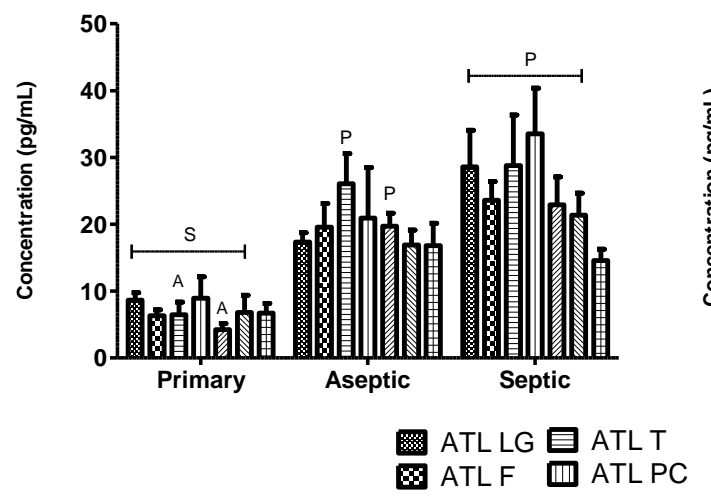

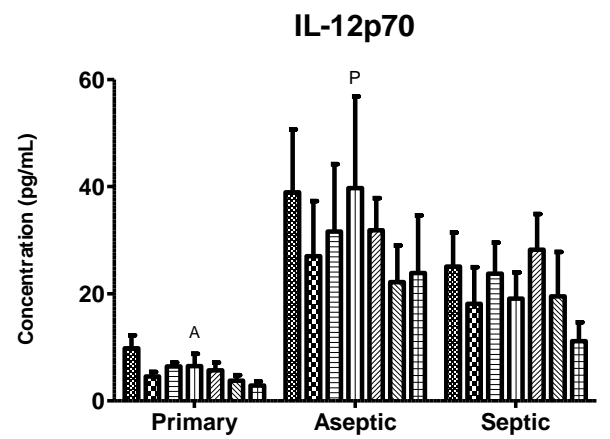

IL-17A

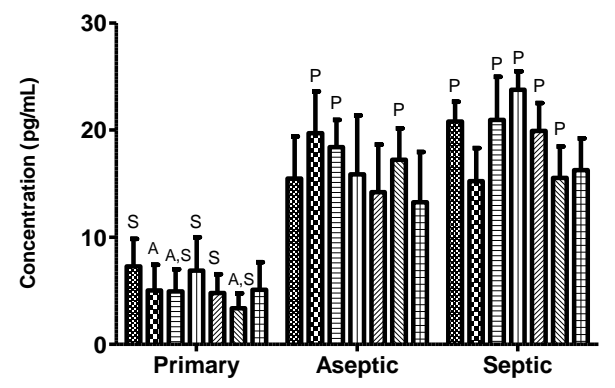

TNF- $\alpha$

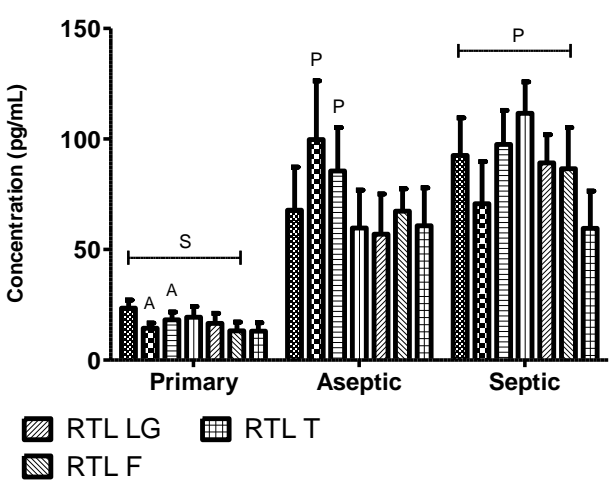

Figure A. 2. Cytokine profiles in localized tissues in implant-related inflammation. Average cytokine concentration at individual tissue locations are shown for all groups. Two-way ANOVAs with Bonferroni's post-test were conducted to test for significant differences between groups at each tissue depth $(\mathrm{p}<0.05)$. Significant differences between groups at a particular location are marked as: $\mathrm{P}$ denotes significant difference from primary TKA $(\mathrm{N}=6)$; A denotes significant difference from aseptic TKR ( $=5)$; $S$ denotes significant difference from septic TKR $(\mathrm{N}=6)$; all symbols denote significance at the $\mathrm{p}<0.05$ level. 


\section{CYTOKINE CALIBRATION CURVES}
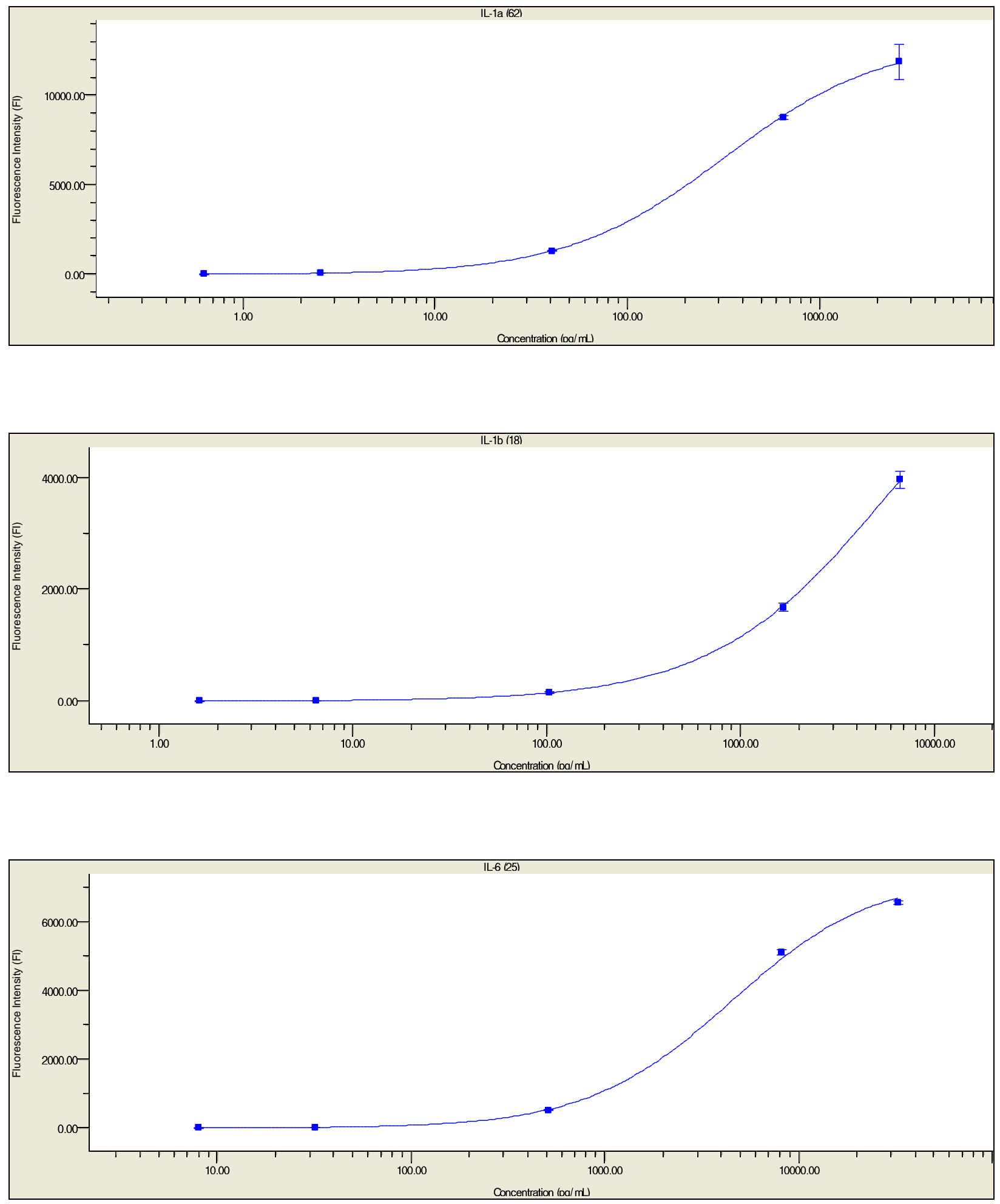

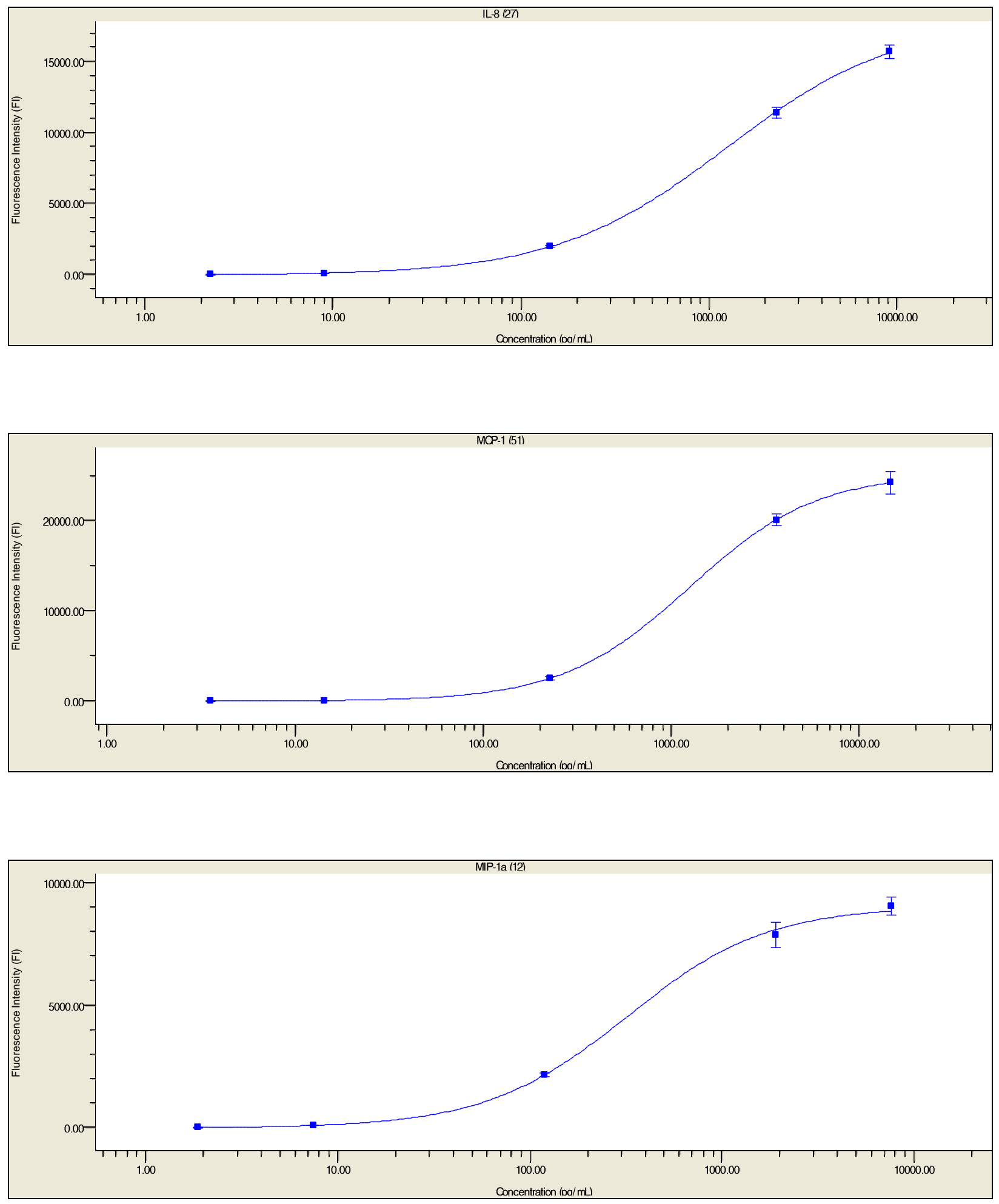

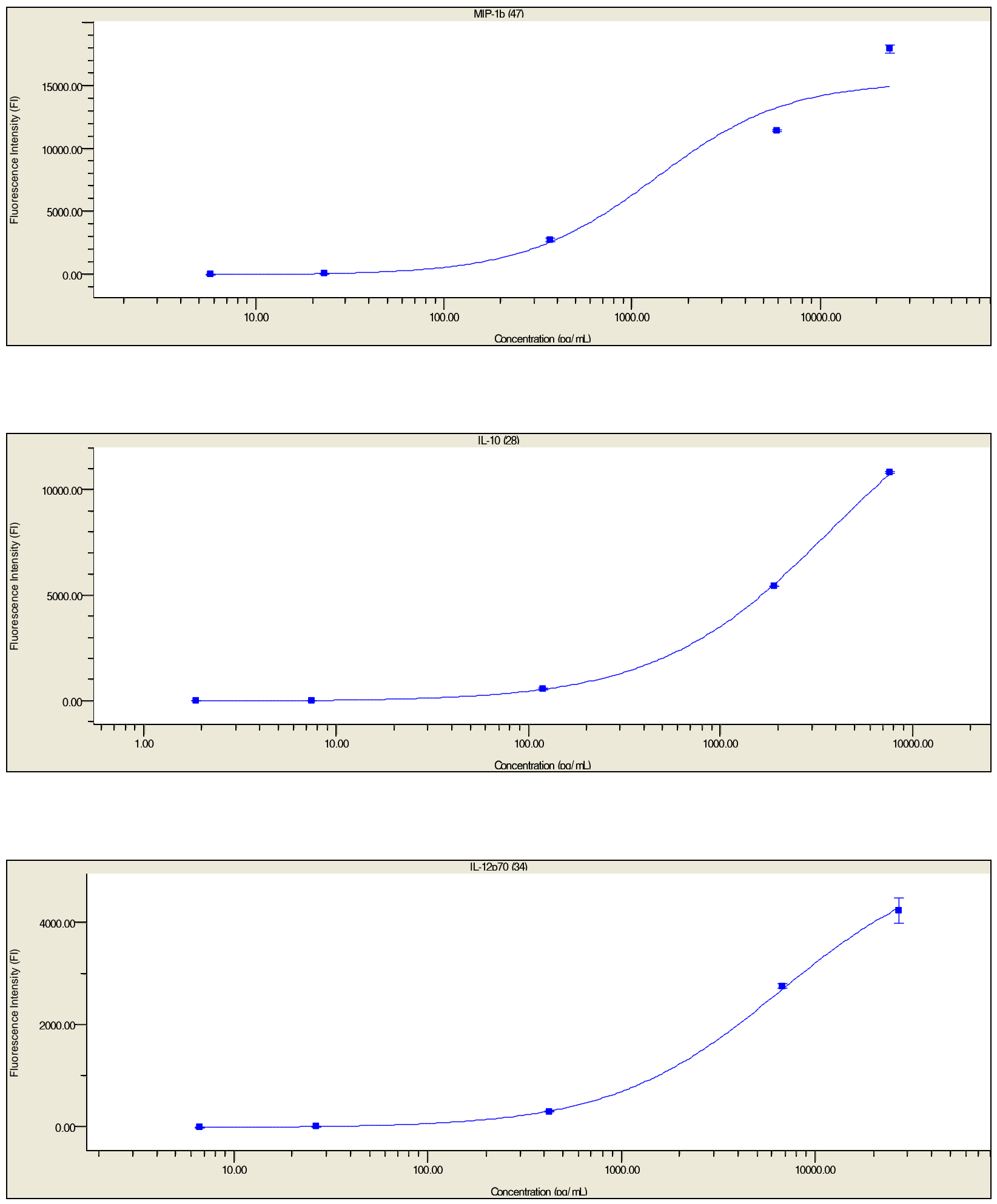

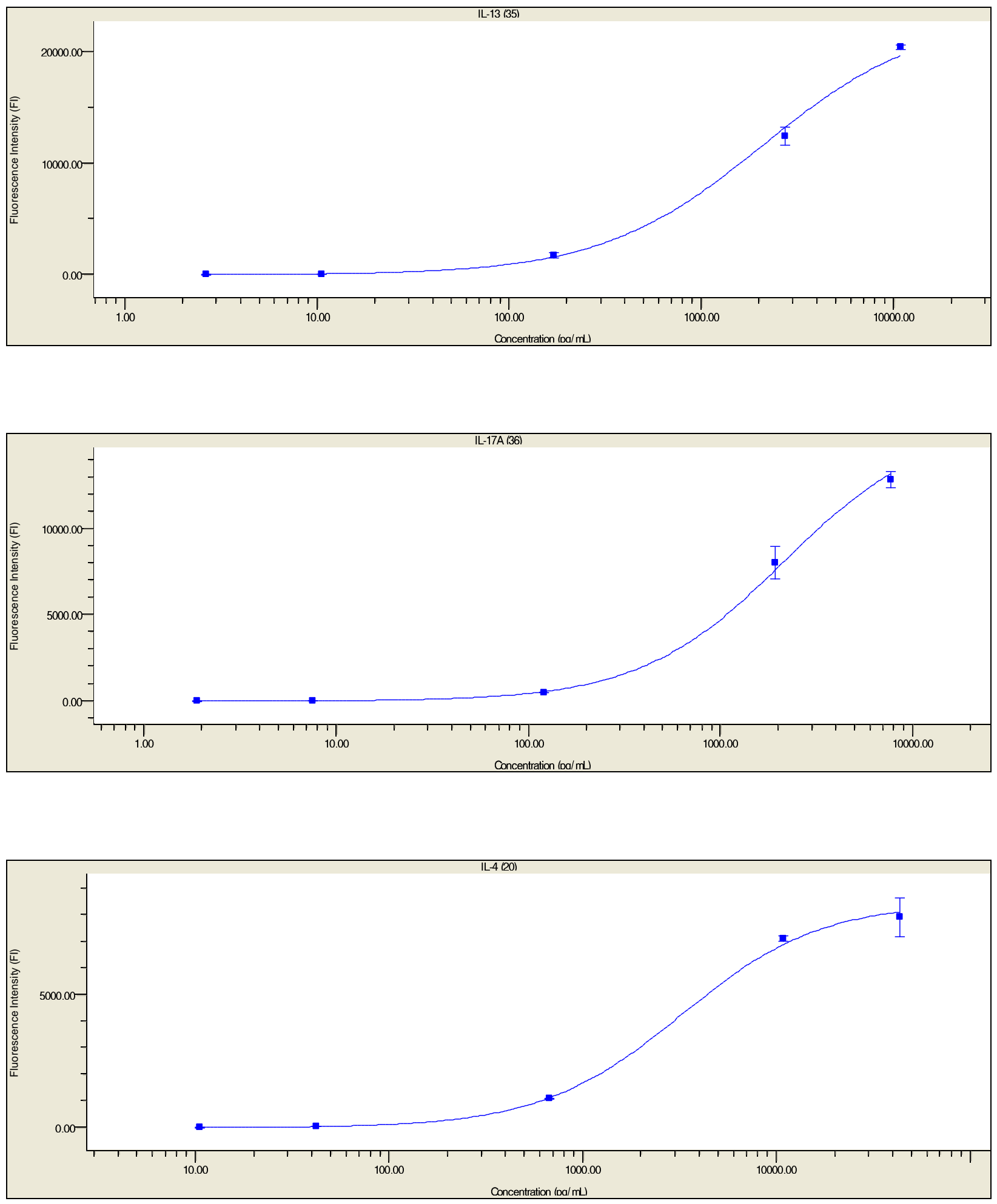


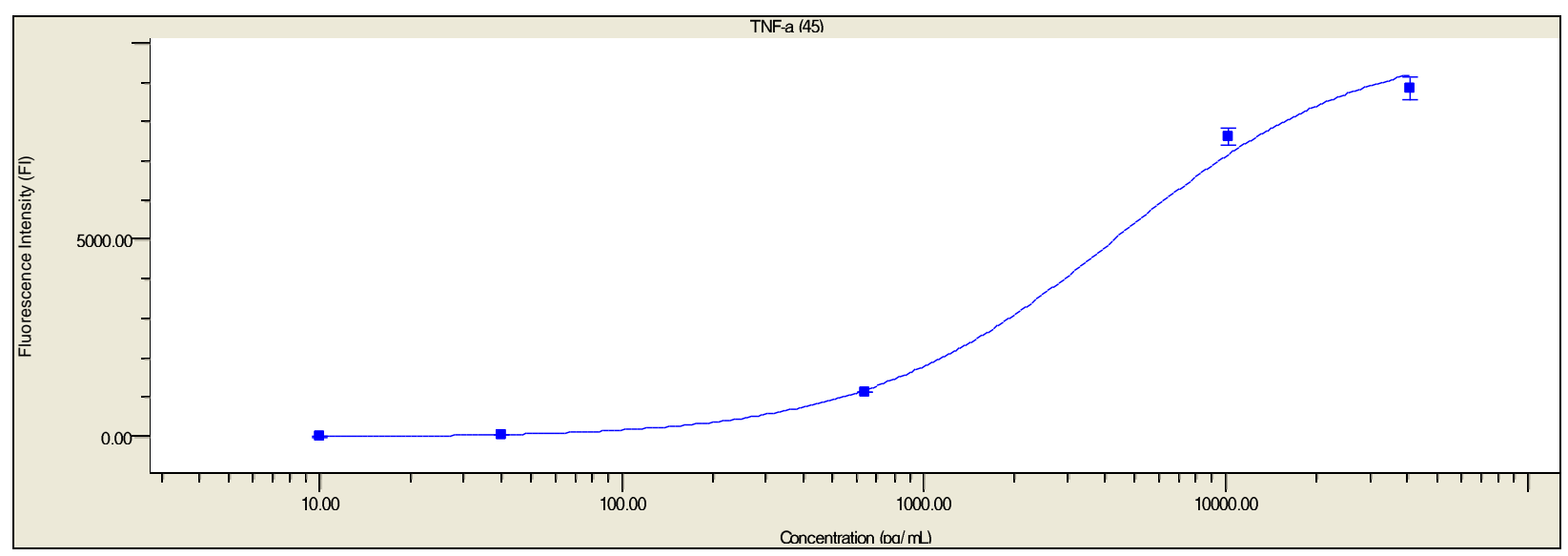

Figure A. 3. Cytokine calibration curves for human inflammatory cytokines. Calibration curves for cytokines were calculated using a four-parameter logistic (4PL) model in Bio-Plex Manager 4.1.1. Five calibration standards were used for each cytokine, and averages of duplicates are shown with error bars denoting 2 standard deviations from the mean. Each graph title additionally shows a number that indicates the bead region for that target in the Bio-Plex system. 


\section{APPENDIX B: CHAPTER 3 SUPPLEMENTARY FIGURES}

Table B. 1. Cytokine concentrations for Sham, Implant Only, and Inf+Implant groups. Values are expressed as nanograms of cytokine per gram of tissue; average values \pm SEM are listed. Note: all cytokines had associated error, but error is rounded to two decimal places. Significant comparisons $(p<0.05)$ between locations for each cytokine within each group are denoted with letters; contralateral knee: A; operative leg proximal: B; operative knee: C; operative leg distal: D. For ease of reader, the significant comparisons between locations $(\mathrm{p}<0.05)$ are simply marked via superscript letters to denote which locations are different. All comparisons were done via two-way ANOVA followed by Bonferroni’s post-test.

\begin{tabular}{|c|c|c|c|c|c|c|c|c|c|c|c|c|}
\hline & \multicolumn{4}{|c|}{ Sham } & \multicolumn{4}{|c|}{ Implant Only } & \multicolumn{4}{|c|}{ Inf+Implant } \\
\hline & Contra & Op Prox & Op Knee & Op Dist & Contra & Op Prox & Op Knee & Op Dist & Contra & Op Prox & Op Knee & Op Dist \\
\hline IL-1 $\alpha$ & $\begin{array}{c}0.07 \pm \\
0.03\end{array}$ & $\begin{array}{c}0.30 \pm \\
0.13\end{array}$ & $\begin{array}{c}0.22 \pm \\
0.09\end{array}$ & $\begin{array}{c}0.26 \pm \\
0.03\end{array}$ & $\begin{array}{c}0.50 \pm \\
0.09^{\mathbf{C}, \mathbf{D}}\end{array}$ & $\begin{array}{l}0.54 \pm \\
0.17^{\mathrm{C}}\end{array}$ & $\begin{array}{c}2.07 \pm \\
0.23^{\mathbf{A}, \mathbf{B}}\end{array}$ & $\begin{array}{l}1.58 \pm \\
0.27^{\mathrm{A}}\end{array}$ & $\begin{array}{c}0.26 \pm \\
0.05^{\mathbf{C}, \mathrm{D}}\end{array}$ & $\begin{array}{l}0.77 \pm \\
0.30^{\mathrm{D}}\end{array}$ & $\begin{array}{c}2.09 \pm \\
0.36^{\mathbf{A}, \mathbf{B}}\end{array}$ & $\begin{array}{c}2.54 \pm \\
1.26^{\mathbf{A}, \mathbf{B}}\end{array}$ \\
\hline IL-1及 & $\begin{array}{c}0.02 \pm \\
0.00\end{array}$ & $\begin{array}{c}0.11 \pm \\
0.05\end{array}$ & $\begin{array}{c}0.18 \pm \\
0.00\end{array}$ & $\begin{array}{c}0.20 \pm \\
0.06\end{array}$ & $\begin{array}{l}0.52 \pm \\
0.10^{\mathrm{C}}\end{array}$ & $\begin{array}{c}0.72 \pm \\
0.18^{\mathbf{C}, \mathbf{D}}\end{array}$ & $\begin{array}{c}6.19 \pm \\
0.98^{\mathbf{A}, \mathbf{B}}\end{array}$ & $\begin{array}{l}1.77 \pm \\
0.21^{\mathbf{B}}\end{array}$ & $\begin{array}{l}0.80 \pm \\
0.28^{\mathrm{C}}\end{array}$ & $\begin{array}{c}1.09 \pm \\
0.53^{\mathbf{C}, \mathbf{D}}\end{array}$ & $\begin{array}{l}10.08 \pm \\
0.10^{\mathbf{A}, \mathbf{B}}\end{array}$ & $\begin{array}{l}2.03 \pm \\
0.33^{\mathbf{B}}\end{array}$ \\
\hline IL-4 & $\begin{array}{c}0.11 \pm \\
0.05 \\
\end{array}$ & $\begin{array}{c}0.37 \pm \\
0.08\end{array}$ & $\begin{array}{c}0.07 \pm \\
0.04\end{array}$ & $\begin{array}{c}0.37 \pm \\
0.12 \\
\end{array}$ & $\begin{array}{l}1.35 \pm \\
0.04^{\mathrm{C}} \\
\end{array}$ & $\begin{array}{l}0.82 \pm \\
0.36^{\mathrm{C}} \\
\end{array}$ & $\begin{array}{l}6.93 \pm \\
2.0^{\mathbf{A}, \mathbf{B}} \\
\end{array}$ & $\begin{array}{c}3.77 \pm \\
1.75 \\
\end{array}$ & $\begin{array}{l}1.56 \pm \\
0.58^{\mathrm{C}} \\
\end{array}$ & $\begin{array}{c}0.79 \pm \\
0.68^{\mathbf{C}, \mathbf{D}}\end{array}$ & $\begin{array}{l}13.15 \pm \\
1.98^{\mathrm{A}, \mathrm{B}}\end{array}$ & $\begin{array}{l}1.69 \pm \\
1.64^{\mathbf{B}} \\
\end{array}$ \\
\hline IL-5 & $\begin{array}{c}0.57 \pm \\
0.01 \\
\end{array}$ & $\begin{array}{c}0.07 \pm \\
0.04\end{array}$ & $\begin{array}{c}0.45 \pm \\
0.34 \\
\end{array}$ & $\begin{array}{c}0.14 \pm \\
0.00 \\
\end{array}$ & $\begin{array}{c}1.77 \pm \\
0.32^{\mathrm{C}, \mathrm{D}}\end{array}$ & $\begin{array}{c}1.78 \pm \\
0.34^{\mathrm{C}, \mathrm{D}}\end{array}$ & $\begin{array}{c}5.38 \pm \\
0.70^{\mathbf{A}, \mathbf{B}}\end{array}$ & $\begin{array}{c}4.93 \pm \\
0.89^{\mathbf{A}, \mathbf{B}}\end{array}$ & $\begin{array}{l}1.13 \pm \\
0.29^{\mathrm{C}} \\
\end{array}$ & $\begin{array}{l}1.62 \pm \\
1.07^{\mathrm{C}} \\
\end{array}$ & $\begin{array}{c}5.79 \pm \\
0.70^{\mathbf{A}, \mathbf{B}, \mathbf{D}} \\
\end{array}$ & $\begin{array}{l}1.54 \pm \\
0.35^{\mathrm{C}} \\
\end{array}$ \\
\hline IL-6 & $\begin{array}{c}0.90 \pm \\
0.38\end{array}$ & $\begin{array}{c}0.62 \pm \\
0.00\end{array}$ & $\begin{array}{c}0.29 \pm \\
0.04\end{array}$ & $\begin{array}{c}0.94 \pm \\
0.31\end{array}$ & $\begin{array}{c}1.82 \pm \\
0.98\end{array}$ & $\begin{array}{c}1.20 \pm \\
0.00\end{array}$ & $\begin{array}{c}7.71 \pm \\
1.27\end{array}$ & $\begin{array}{c}7.90 \pm \\
2.81\end{array}$ & $\begin{array}{l}1.17 \pm \\
0.16^{\mathrm{C}}\end{array}$ & $\begin{array}{l}0.73 \pm \\
0.20^{\mathrm{C}}\end{array}$ & $\begin{array}{c}17.27 \pm \\
6.70^{\mathbf{A}, \mathbf{B}, \mathbf{D}}\end{array}$ & $\begin{array}{l}4.15 \pm \\
2.65^{\mathrm{C}}\end{array}$ \\
\hline IL-10 & $\begin{array}{c}0.18 \pm \\
0.12 \\
\end{array}$ & $\begin{array}{c}0.55 \pm \\
0.39 \\
\end{array}$ & $\begin{array}{c}0.41 \pm \\
0.01 \\
\end{array}$ & $\begin{array}{c}0.19 \pm \\
0.12 \\
\end{array}$ & $\begin{array}{c}1.73 \pm \\
0.45^{\mathrm{C}, \mathrm{D}}\end{array}$ & $\begin{array}{c}1.37 \pm \\
0.32^{\mathbf{C}, \mathbf{D}}\end{array}$ & $\begin{array}{c}6.89 \pm \\
1.24^{\mathbf{A , B}}\end{array}$ & $\begin{array}{c}5.71 \pm \\
1.35^{\mathbf{A}, \mathbf{B}}\end{array}$ & $\begin{array}{l}1.17 \pm \\
0.04^{\mathrm{C}} \\
\end{array}$ & $\begin{array}{l}1.33 \pm \\
0.66^{\mathrm{C}} \\
\end{array}$ & $\begin{array}{c}6.43 \pm \\
0.07^{\mathrm{A}, \mathbf{B}, \mathbf{D}} \\
\end{array}$ & $\begin{array}{l}1.30 \pm \\
0.80^{\mathrm{C}} \\
\end{array}$ \\
\hline IL-12p70 & $\begin{array}{c}0.98 \pm \\
0.27 \\
\end{array}$ & $\begin{array}{c}1.58 \pm \\
0.43 \\
\end{array}$ & $\begin{array}{c}1.58 \pm \\
0.39 \\
\end{array}$ & $\begin{array}{c}1.93 \pm \\
0.51 \\
\end{array}$ & $\begin{array}{c}5.38 \pm \\
0.95^{\mathrm{C}, \mathrm{D}}\end{array}$ & $\begin{array}{c}5.60 \pm \\
1.25^{\mathrm{C}, \mathrm{D}}\end{array}$ & $\begin{array}{l}25.51 \pm \\
2.96^{\mathbf{A}, \mathbf{B}}\end{array}$ & $\begin{array}{l}17.37 \pm \\
4.49^{\mathbf{A}, \mathbf{B}}\end{array}$ & $\begin{array}{l}5.58 \pm \\
0.62^{\mathrm{C}} \\
\end{array}$ & $\begin{array}{l}3.93 \pm \\
2.81^{\mathrm{C}} \\
\end{array}$ & $\begin{array}{l}18.14 \pm \\
5.08^{\mathrm{A}, \mathrm{B}}\end{array}$ & $\begin{array}{c}6.98 \pm \\
1.38 \\
\end{array}$ \\
\hline IL-13 & $\begin{array}{c}0.81 \pm \\
0.30 \\
\end{array}$ & $\begin{array}{c}0.26 \pm \\
0.12 \\
\end{array}$ & $\begin{array}{c}0.39 \pm \\
0.10 \\
\end{array}$ & $\begin{array}{c}0.35 \pm \\
0.00 \\
\end{array}$ & $\begin{array}{l}2.03 \pm \\
0.74^{\mathrm{C}} \\
\end{array}$ & $\begin{array}{c}1.32 \pm \\
0.27^{\mathbf{C}, \mathbf{D}}\end{array}$ & $\begin{array}{c}6.58 \pm \\
1.56^{\mathrm{A}, \mathrm{B}} \\
\end{array}$ & $\begin{array}{l}4.89 \pm \\
1.11^{\mathbf{B}} \\
\end{array}$ & $\begin{array}{l}1.50 \pm \\
0.28^{\mathrm{C}} \\
\end{array}$ & $\begin{array}{l}1.22 \pm \\
0.30^{\mathrm{C}} \\
\end{array}$ & $\begin{array}{c}6.20 \pm \\
0.95^{\mathrm{A}, \mathbf{B}, \mathbf{C}} \\
\end{array}$ & $\begin{array}{l}1.47 \pm \\
0.03^{\mathrm{C}} \\
\end{array}$ \\
\hline GM-CSF & $\begin{array}{c}0.17 \pm \\
0.06\end{array}$ & $\begin{array}{c}0.23 \pm \\
0.02 \\
\end{array}$ & $\begin{array}{c}0.32 \pm \\
0.09 \\
\end{array}$ & $\begin{array}{c}0.64 \pm \\
0.15 \\
\end{array}$ & $\begin{array}{l}1.08 \pm \\
0.29^{\mathrm{C}} \\
\end{array}$ & $\begin{array}{l}0.52 \pm \\
0.22^{\mathrm{C}} \\
\end{array}$ & $\begin{array}{c}6.65 \pm \\
1.23^{\mathbf{A}, \mathbf{B}, \mathbf{D}} \\
\end{array}$ & $\begin{array}{l}2.56 \pm \\
0.98^{\mathrm{C}} \\
\end{array}$ & $\begin{array}{l}1.09 \pm \\
0.26^{\mathrm{C}} \\
\end{array}$ & $\begin{array}{l}0.86 \pm \\
0.66^{\mathrm{C}} \\
\end{array}$ & $\begin{array}{c}5.85 \pm \\
0.47^{\mathbf{A}, \mathbf{B}, \mathbf{D}} \\
\end{array}$ & $\begin{array}{l}0.90 \pm \\
0.64^{\mathrm{C}} \\
\end{array}$ \\
\hline IFN- $\gamma$ & $\begin{array}{c}0.49 \pm \\
0.24\end{array}$ & $\begin{array}{c}0.50 \pm \\
0.09\end{array}$ & $\begin{array}{c}0.92 \pm \\
0.31 \\
\end{array}$ & $\begin{array}{c}0.76 \pm \\
0.18\end{array}$ & $\begin{array}{l}3.87 \pm \\
0.78^{\mathrm{C}} \\
\end{array}$ & $\begin{array}{c}6.40 \pm \\
2.74 \\
\end{array}$ & $\begin{array}{c}10.97 \pm \\
3.04^{\mathrm{A}}\end{array}$ & $\begin{array}{c}9.14 \pm \\
1.83 \\
\end{array}$ & $\begin{array}{l}2.84 \pm \\
0.23^{\mathrm{C}} \\
\end{array}$ & $\begin{array}{l}2.83 \pm \\
1.78^{\mathrm{D}} \\
\end{array}$ & $\begin{array}{c}12.57 \pm \\
3.13^{\mathrm{A}}\end{array}$ & $\begin{array}{l}5.04 \pm \\
1.73^{\mathbf{B}} \\
\end{array}$ \\
\hline TNF- $\alpha$ & $\begin{array}{c}0.85 \pm \\
0.36 \\
\end{array}$ & $\begin{array}{c}1.87 \pm \\
0.51 \\
\end{array}$ & $\begin{array}{c}1.53 \pm \\
0.01 \\
\end{array}$ & $\begin{array}{c}3.96 \pm \\
0.85 \\
\end{array}$ & $\begin{array}{c}4.66 \pm \\
1.16 \\
\end{array}$ & $\begin{array}{l}3.08 \pm \\
0.70^{\mathrm{C}} \\
\end{array}$ & $\begin{array}{c}11.11 \pm \\
3.03^{\mathrm{B}}\end{array}$ & $\begin{array}{c}7.41 \pm \\
2.3 \\
\end{array}$ & $\begin{array}{c}5.78 \pm \\
2.56 \\
\end{array}$ & $\begin{array}{c}4.97 \pm \\
4.21 \\
\end{array}$ & $\begin{array}{c}9.79 \pm \\
3.35 \\
\end{array}$ & $\begin{array}{c}6.02 \pm \\
2.42 \\
\end{array}$ \\
\hline
\end{tabular}




\section{X-RAY IMAGES OF RODENTS AT 21 DAYS POST-SURGERY}

\section{Methods}

Grading and Analysis of X-Rays

X-rays were taken on the day of operation as well as 21 days post-operation to compare joint conditions. X-rays at each time point were graded using methods established by Aktekin, et al. to evaluate the following parameters: periosteal reaction, diaphyseal widening, osteolysis, bone formation, sequestrum, joint effusion, and swelling. Results for each of the three groups: Sham, Implant Only, and Inf+Implant are displayed in Table S2. Representative X-ray images for each group are displayed, labeled with group. X-rays are shown at 21 days post-operation, and a red arrow points to the operative right knee joint. For some animals, staples can be seen on the right hind limb. For Implant Only and Inf+Implant groups, K-wire implant is denoted at the red arrow.

\section{X-Ray Analysis, Grading, and Results}

Table B. 2. $X$-Ray Grading Scores for Sham $(N=6)$, Implant Only $(N=6)$, and Inf + Implant $(N=6)$ Groups at Post-Operative Day 0 (POD-0) and POD-21. X-rays were graded according to methods established by Aktekin, et al.

\begin{tabular}{|c|c|c|c|c|c|c|}
\hline & \multicolumn{2}{|c|}{ SHAM } & \multicolumn{2}{c|}{ IMPLANT ONLY } & \multicolumn{2}{c|}{ INF+IMPLANT } \\
\hline & POD 0 & POD 21 & POD 0 & POD 21 & POD 0 & POD 21 \\
\hline Periosteal reaction & 0.04167 & 0.08333 & 0.04167 & 0.08333 & 0 & 1.08333 \\
\hline Diaphyseal widening & 0 & 0 & 0 & 0.125 & 0 & 0.875 \\
\hline Osteolysis & 0.08333 & 0.08333 & 0.04167 & 0.20833 & 0 & 1.20833 \\
\hline Bone formation & 0 & 0 & 0 & 0.04167 & 0 & 0.75 \\
\hline Sequestrum & 0 & 0 & 0 & 0 & 0 & 0.16667 \\
\hline Joint effusion & 0 & 0 & 0 & 0 & 0 & 0.04167 \\
\hline Swelling & 0 & 0 & 0 & 0 & 0 & 0 \\
\hline
\end{tabular}


X-Ray Images of Sham, Implant Only, and Inf+Implant Groups

Sham Group X-rays at 21-day Time Point
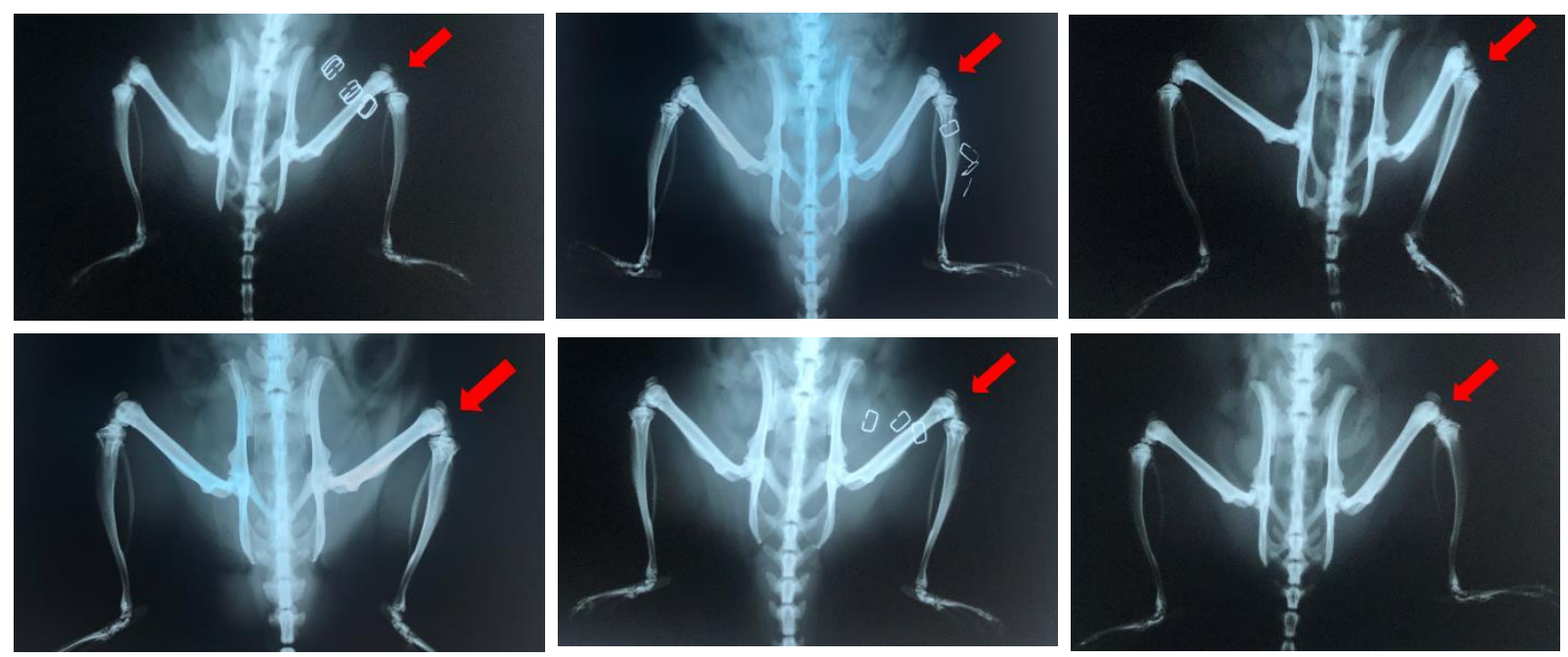

Figure B. 1. X-ray images of Sham group rats at 21 days post-surgery. Radiographs are shown of animals in the sham group. In some animals, staples can be seen. Red arrows indicate operative knee.

Implant Only Group X-rays at 21-day Time Point
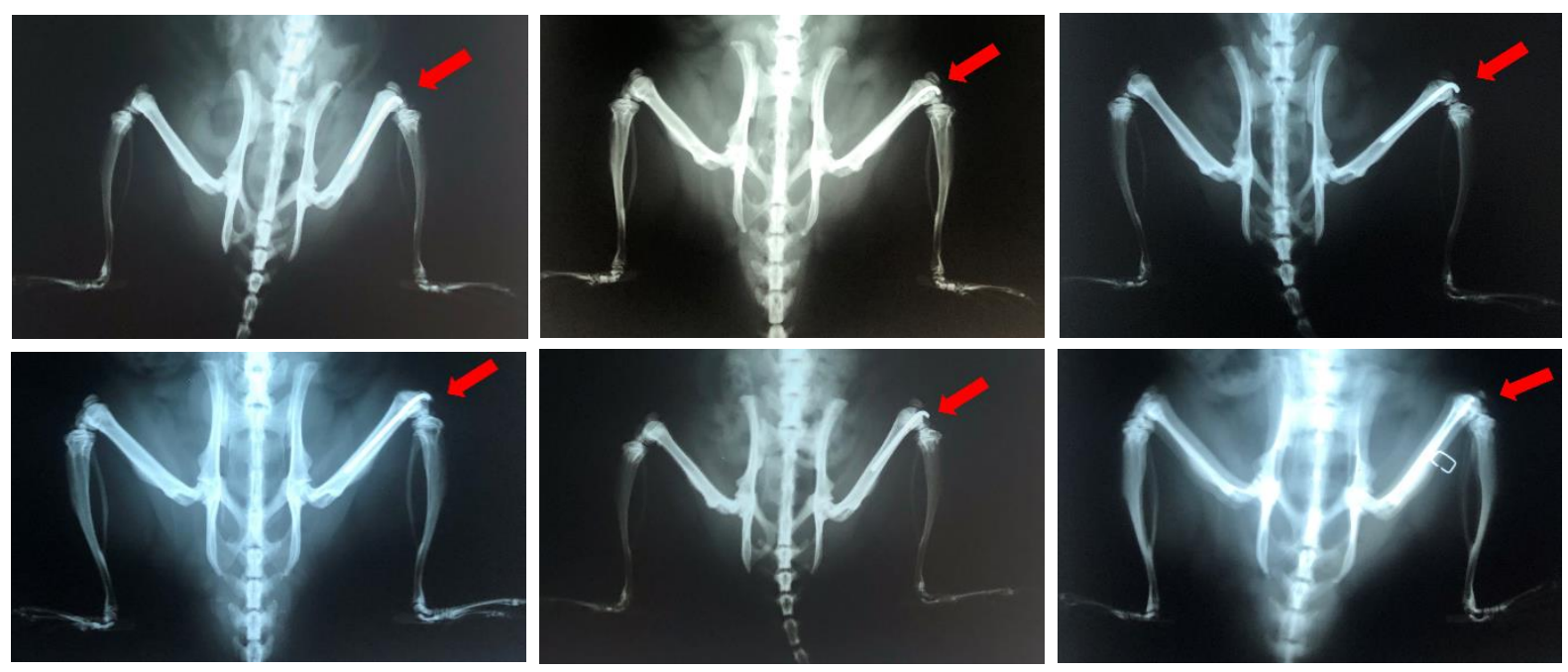

Figure B. 2. X-ray images of Impant Only group rats at 21 days post-surgery. Radiographs are shown of animals in the Implant Only group. In some animals, staples can be seen. Red arrows indicate operative knee. 
Inf+Implant Only Group X-rays at 21-day Time Point
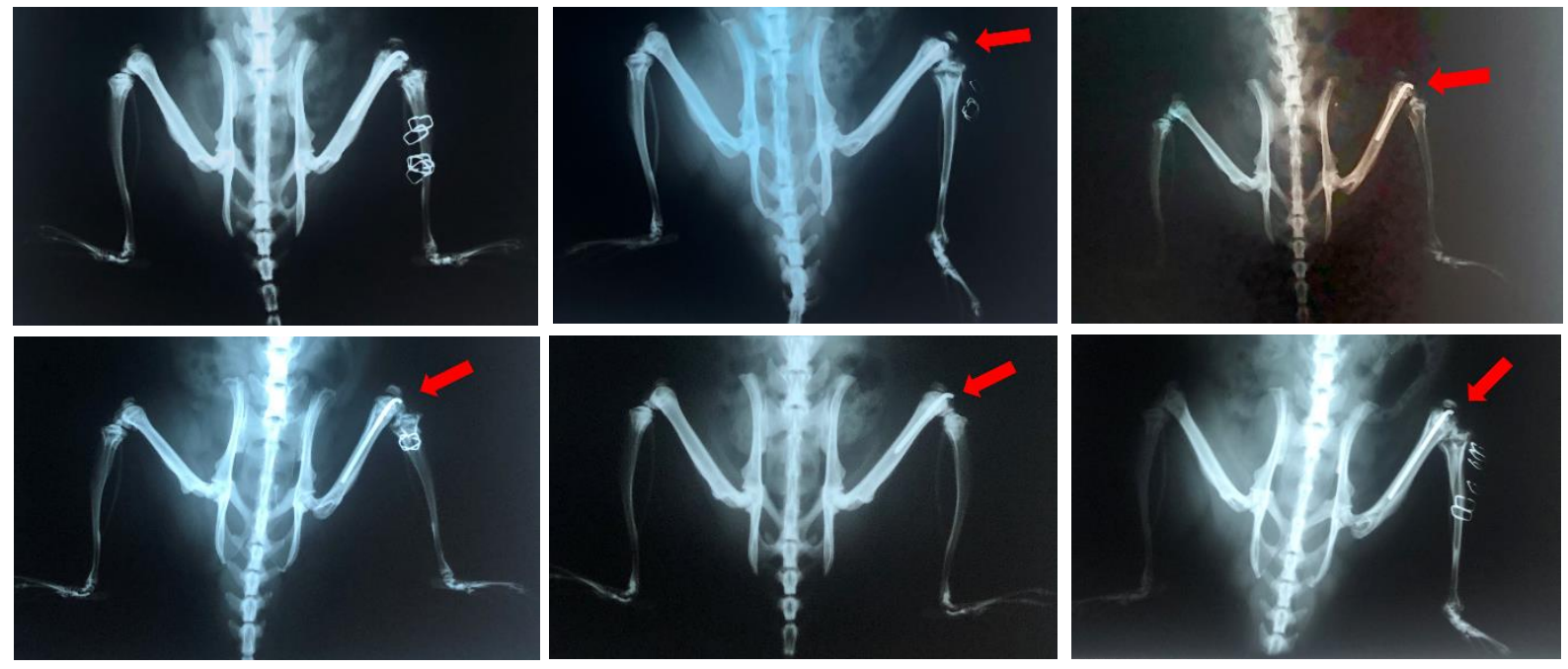

Figure B. 3. X-ray images of Inf+Impant group rats at 21 days post-surgery. Radiographs are shown of animals in the Inf+Implant group. In some animals, staples can be seen. Red arrows indicate operative knee. 


\section{CYTOKINE CONCENTRATIONS IN SERUM OF RODENTS AT 21 DAYS POST- OPERATION}
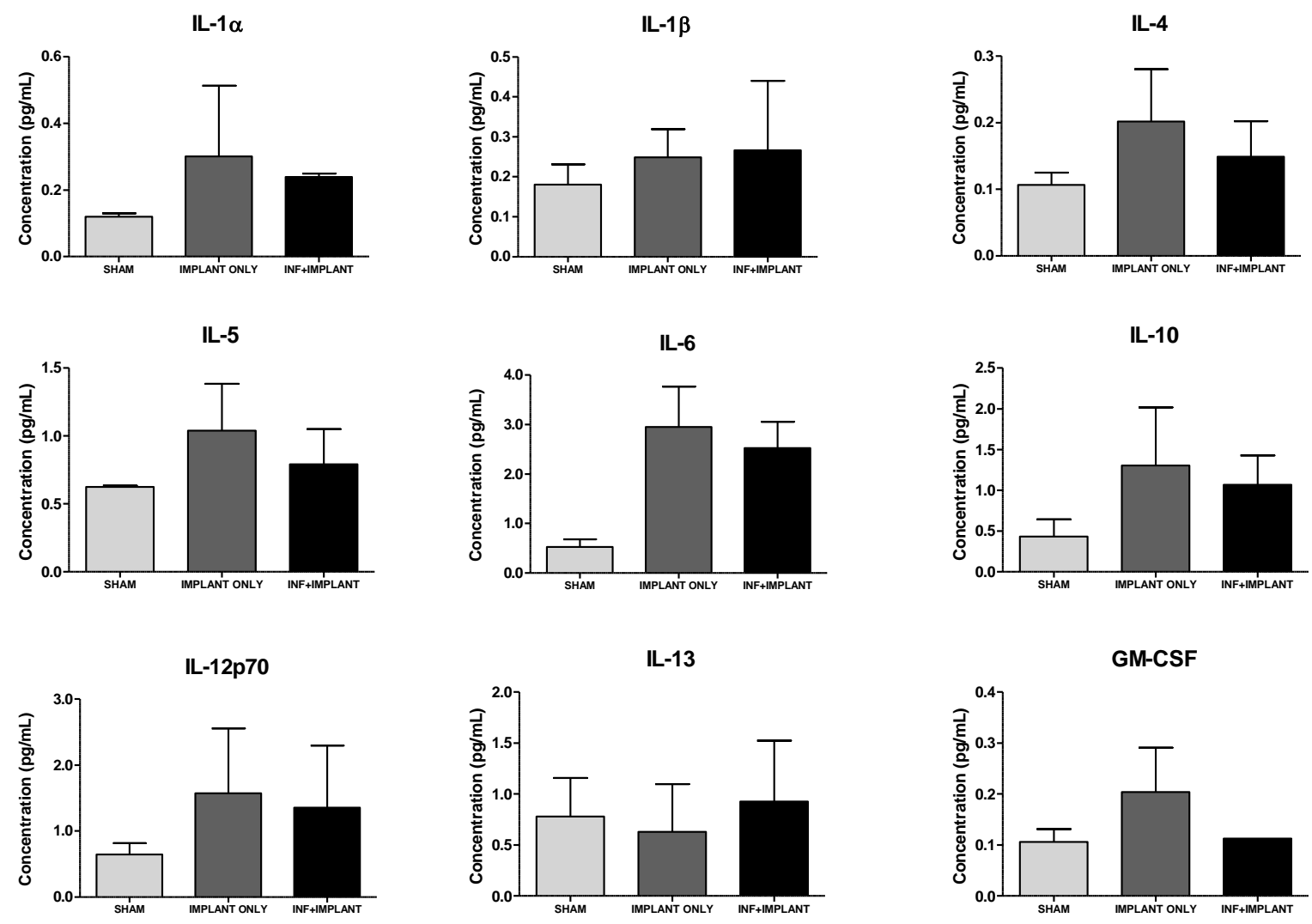

IL-13
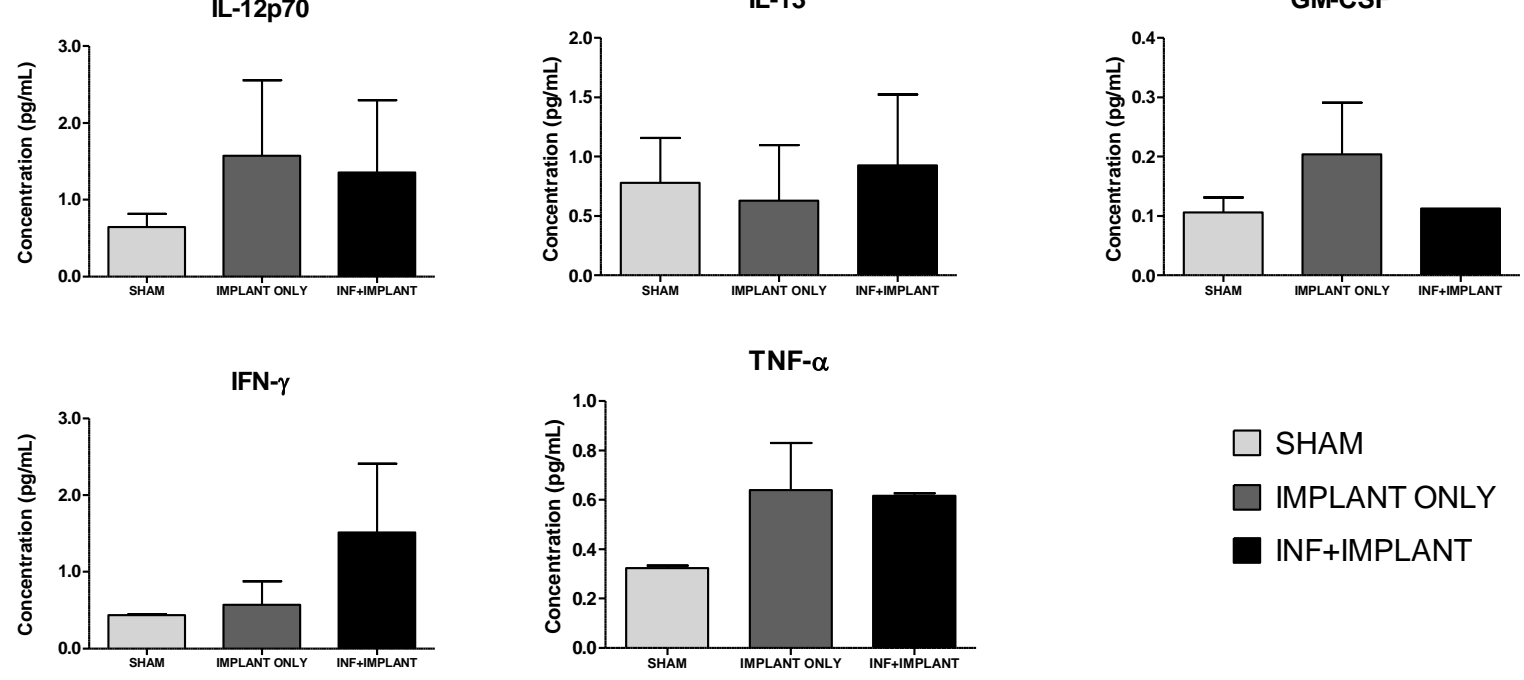

Figure B. 4. Cytokine profiles in localized tissues in infection-specific inflammation. Average cytokine concentration in the serum of rats at the 21 day time point are shown for Sham, Implant Only, and Inf+Implant groups. Two-way ANOVAs with Bonferroni's post-test were conducted to test for significant differences between groups, but no significant differences were observed at $\mathrm{p}<0.05$. 


\section{CYTOKINE CALIBRATION CURVES}
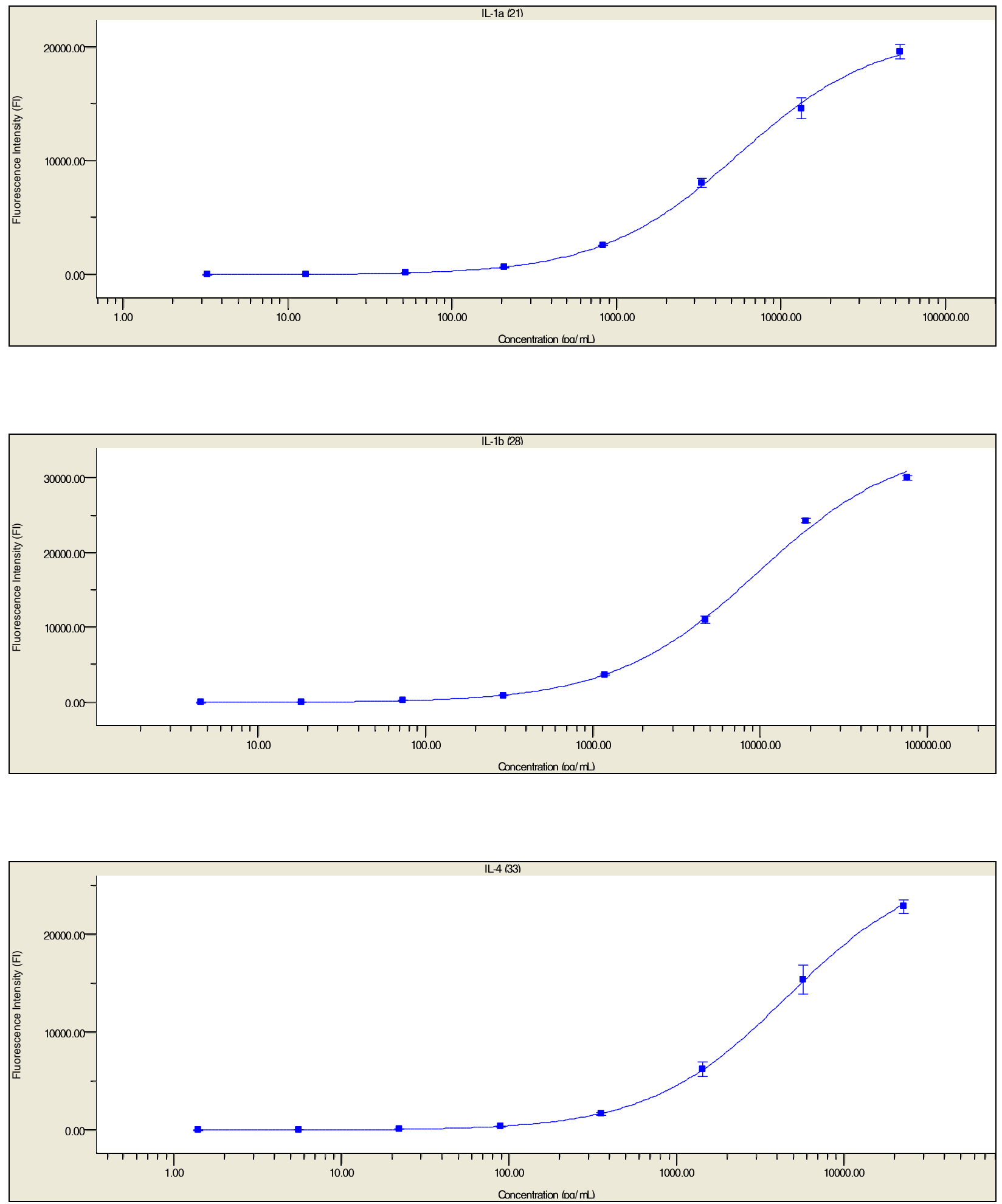

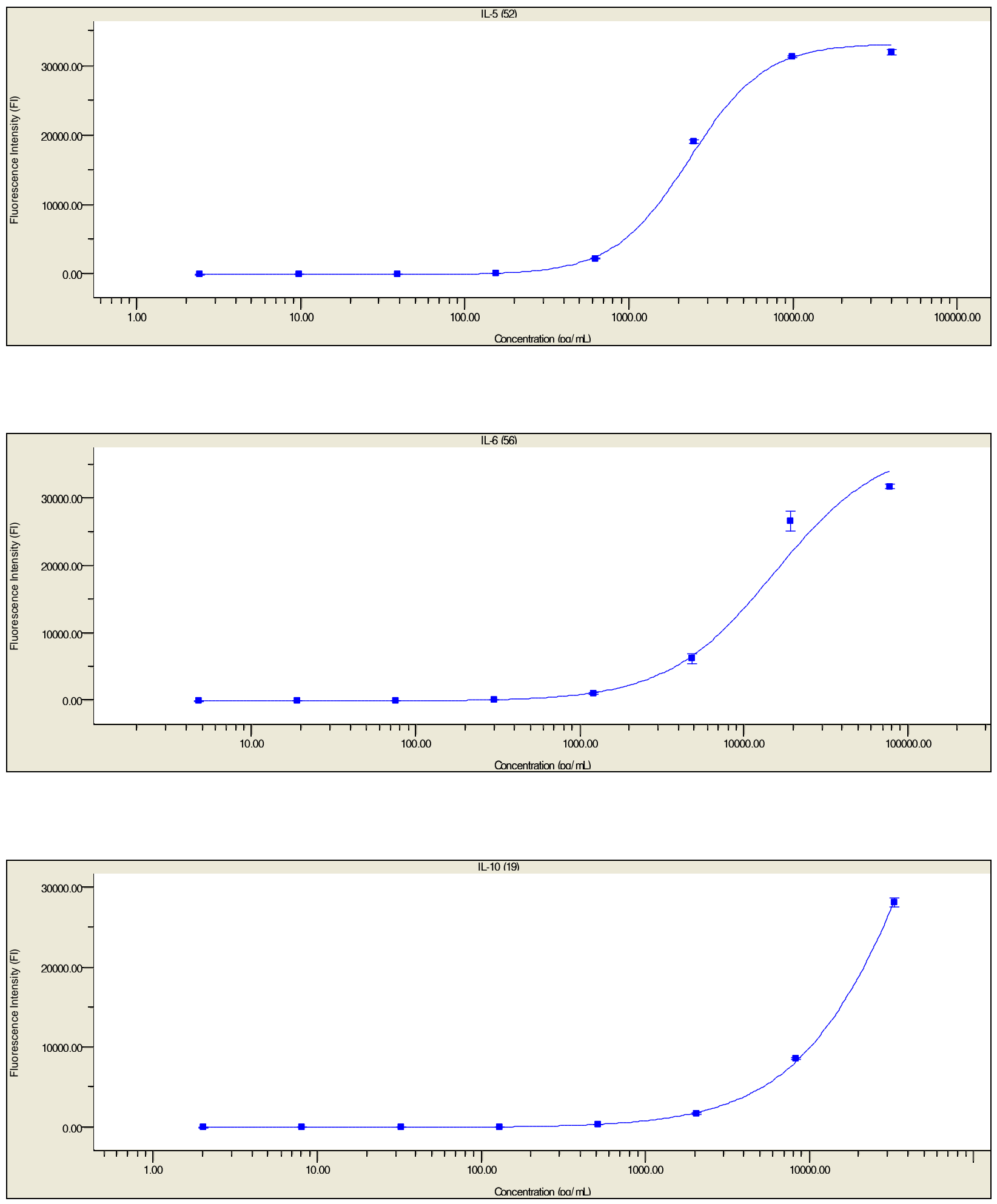

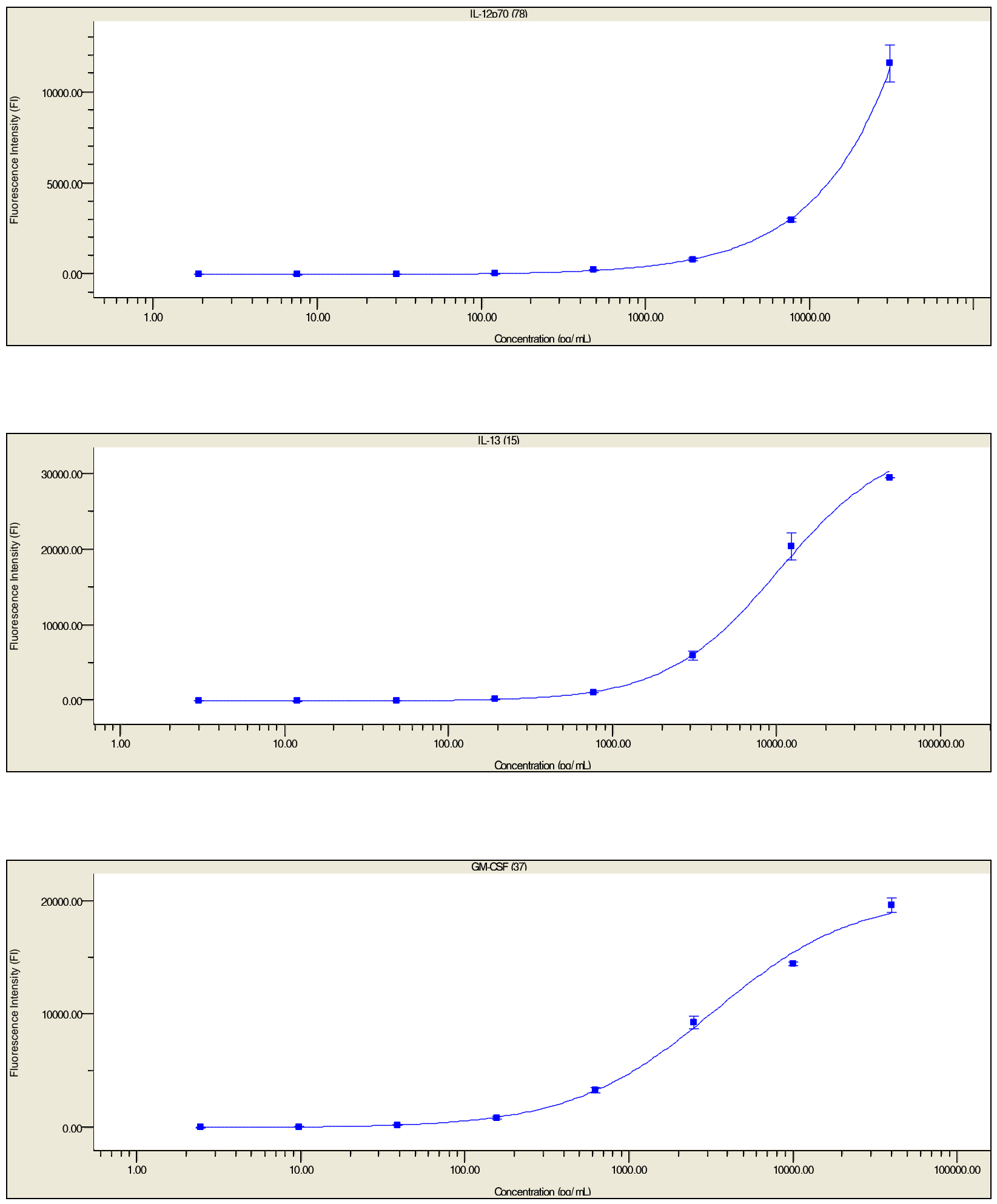

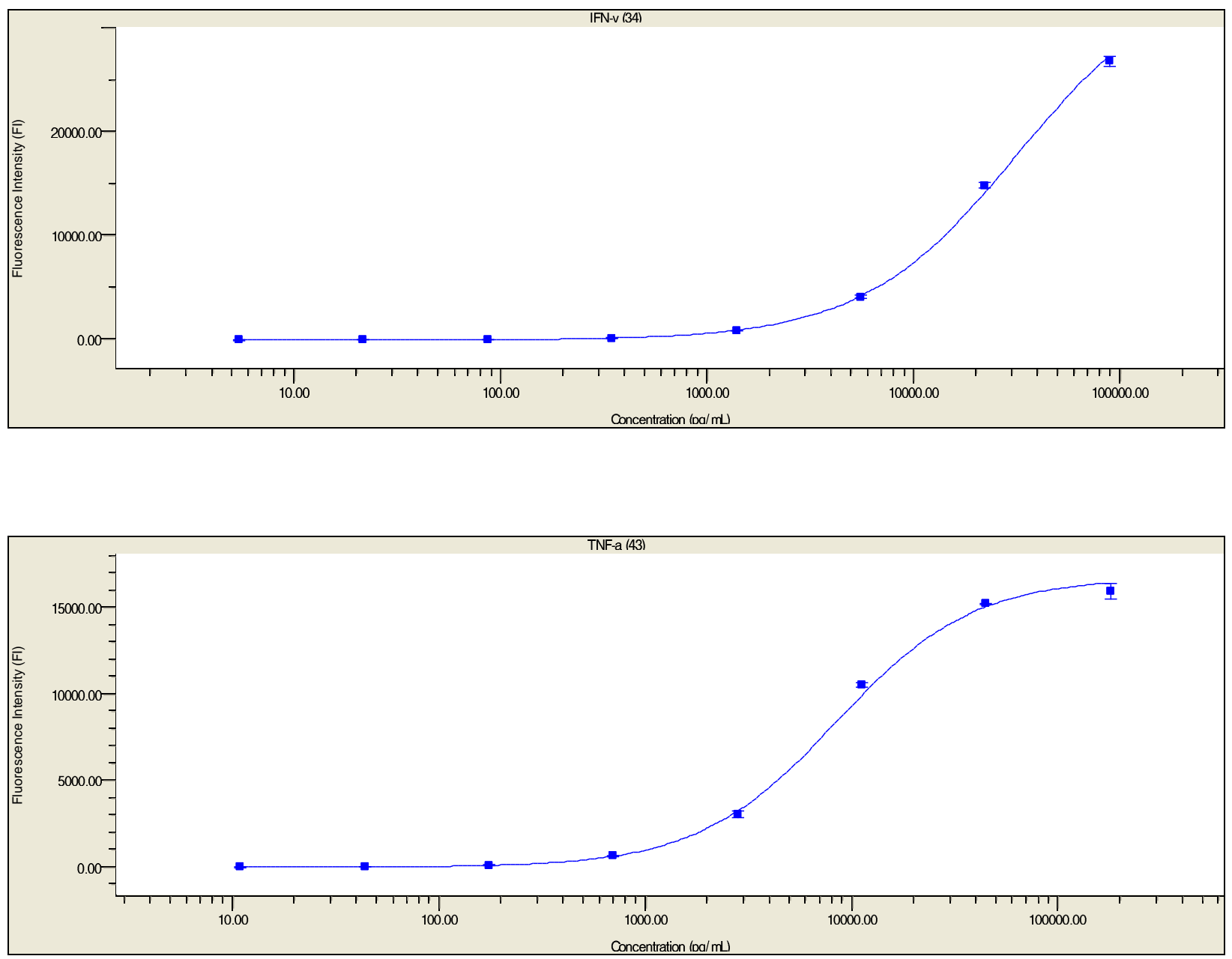

Figure B. 5. Cytokine calibration curves for rodent inflammatory cytokines. Calibration curves for cytokines were calculated using a four-parameter logistic (4PL) model in Bio-Plex Manager 4.1.1. Five calibration standards were used for each cytokine, and averages of duplicates are shown with error bars denoting 2 standard deviations from the mean. Each graph title additionally shows a number that indicates the bead region for that target in the Bio-Plex system. 